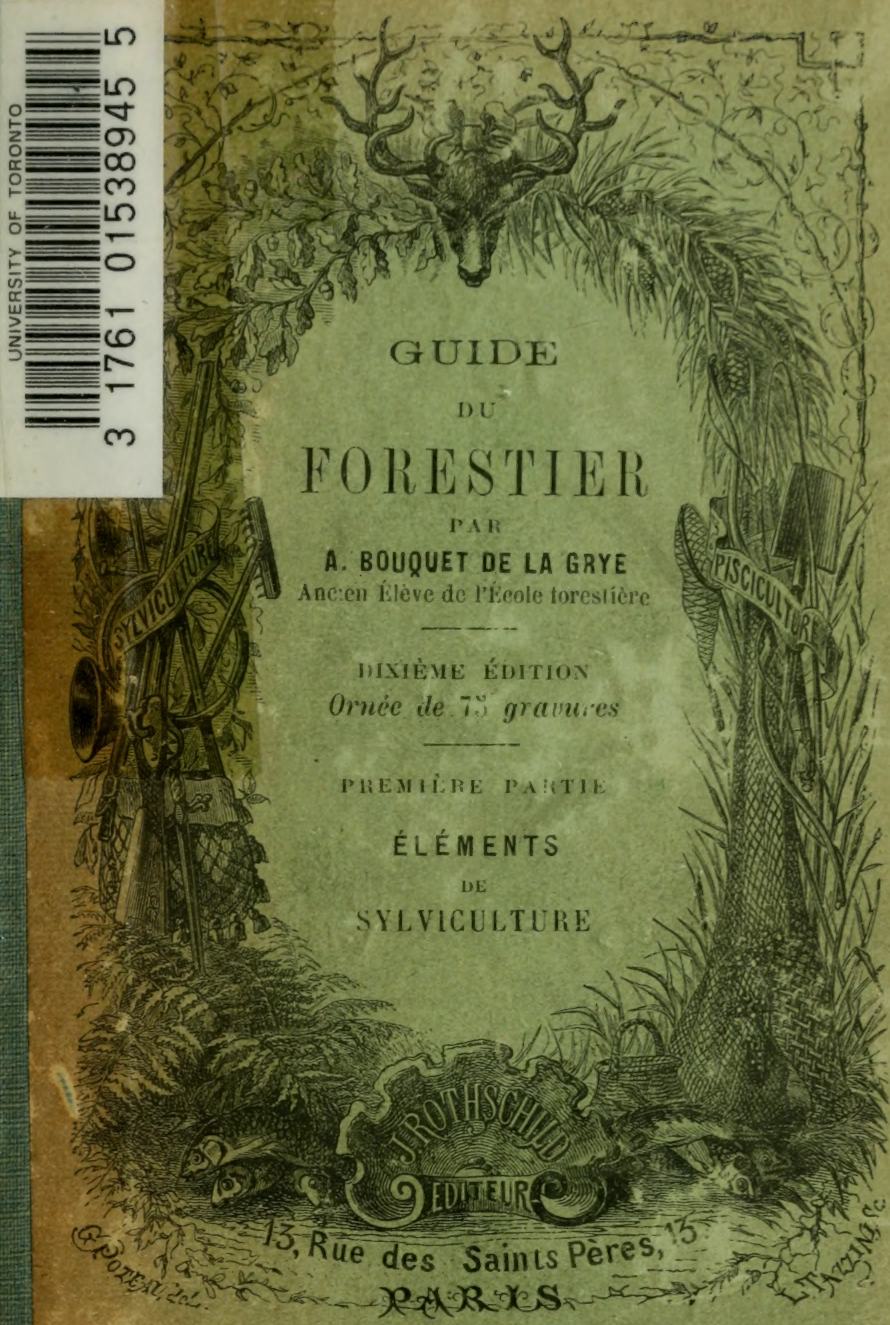





\title{
GUIDE
}

\section{DU FORESTIER}

PREMIÈRE PARTIE

ÉLÉIIENTS DE SYLVIGULTURE

\author{
LIBRARY \\ FACULTY OF FORESTRY \\ UNIVERSITY OF TORONTO
}


26-12-8. - Tours, imp. Anrault el Cie. 


\section{ÉLÉMENTS}

1) $\mathrm{E}$

SYLVICULTURE

P A R

A. BOUQUET DE LA GRYE Ancien conserrateur des Forêts

Jiembre de la Société centrale d'Agriculture de France

AVEC SOIXANTE-QUINZE VIGNETTES

$$
\text { DIXIËME ÉDITION }
$$

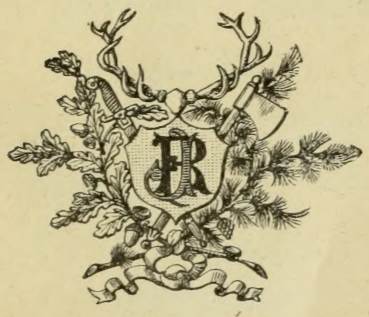

$$
\text { PAR I S }
$$

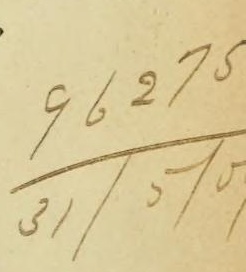

J. ROT H S CH I L D, É D I T E U R

13, RUE DES SAINTS-PÈRES, 13

1899 


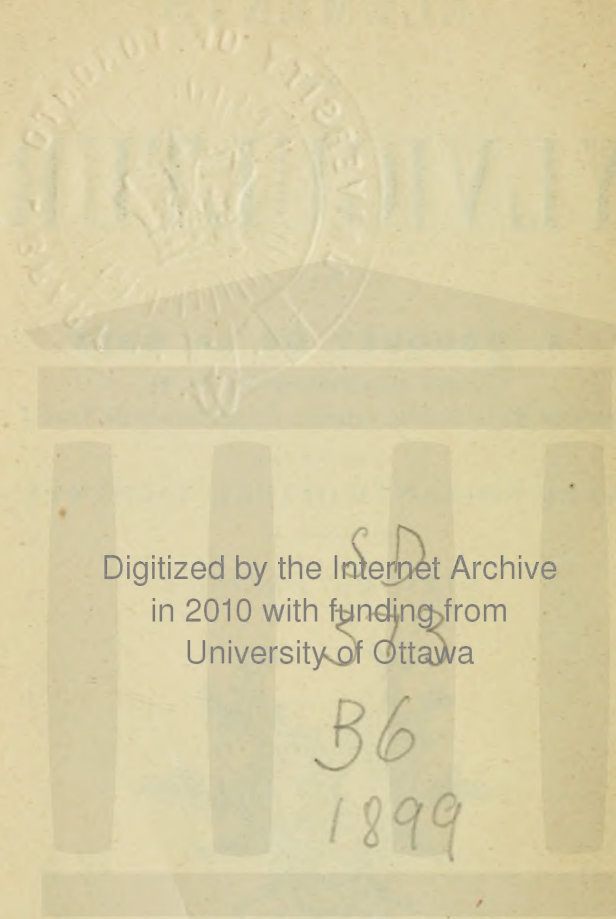

http://www.archive.org/details/lmentsdesylv00bouq 


\section{SOMMAIRES}

\section{LES MILIEUX}

\section{I. - LE GLOBE TERRESTRE}

Forme. - Dimensions. - Refroidissement. - Soulève ments. - Premières couches sédimentaires. - Fossiles. - Chaines de montagnes. - Leur altitude. - Vallées. - Lacs. . . . 1 à 8

\section{1i. - L'ATMOSPHÈRE}

Épaisseur. - Composition. - Oxygène. - Azote. - Acide carbonique. - Carbone. - Origine de l'acide carbonique. - Vapeur d'eau . . . . . . . . 9 à 15

\section{III. - L'EAU}

Composition de l'eau. - Hydrogène. - Sources, rivières et fieuves. - Pluie. - Neige. - Glaciers. - Eaux douces, salines et minérales. - Ammoniaque et acide azotique . . . . . . . . . . . . 16 à 23

\section{IV. - LE SOL}

Sols arables.-Solsd'alluvion etde désagrégation.-Roches ignées, sédimentaires et métamorphiques. - Granits, talcs et quartz. - Calcaires, grès, argiles. - Humus. Définitions. - Effets de la culture . . . . 24 à 36

\section{PHYSIOLOGIE}

\section{V. - NUTRITION}

Germination. - Conditions favorables. - Cellules, fibres etvaisseaux. - Composition d'une jeune tige. - Racines. - Feuilles. - Boutons. - Circulation de la sève ascendante. - Transformation dans les feuilles. - Assimilation du carbone. - Action de la lumière. - Sève descendante. - Cambium. - Formation des tissus. Accroissement de la tige et des racines. - Effets du couvert. - Périodes de la vie des arbres. - Jeunesse, maturité, décrépitude et mort naturelle. - Des maladies et de la mort anticipée . . . . . . . 37 à 77 


\section{VI - REPRODUCTION}

Boutons. - Bourgeons terminaux, latéraux, adventifs. Rejets de souche. - Fleur. - Etamines. - Pistils. Fécondation. - Fructification. - Diverses formes des graines. - Dissémination .. . . . . 78 à 94

\section{SYLVICULTURE}

\section{VII. - TAILLIS}

Traitement. - Taillis simpleset composés. - Réserves. Essences propres aux taillis. - Sols et climats. 95 à 99 Aménagement. - Durée de la révolution. - Assiette de l'aménagement. - Division en coupes, immédiate, successive. - Voies de vidanges . . . 100 à 109 Exploitation. - Abatage - Causes de dépérissement des souches. - Nécessité de couper rez tronc. - Exceptions. - Saisons de l'abatage. - Faconnage. - Vidange . . . . . . . 109 à 116 Réserves. - Importance du balivage. - Choix des réserves, baliveaux modernes, anciens . . . 116 à 119 Entretien. - Nettoiements. - Restauration des taillis ruinés, par semis de pins et de chènes, par plantations. . . . . . . . 119 à 124 Cultures spéclales. - Furetage. - Sartage. - Ecorces à tan. - Liège

\section{VIII. - FUTAIES}

Modes de traitement. - Méthode naturelle. - Jardinage. - Tire el aire. - Blanc-étoc. - Gemmage du pin maritime . . . . . . . . . . 132 à 133

Méthode naturelle. - Coupes de régénération : sombres. claires, secondaires, définitives - Coupes d'amélioration: Nettoiements, éclaircies. - Exploitabilité. Possibilité. - Marche des exploitations. - Repeuplements artificiels. - Abatage. - Vidange. . 133 à 151 Jardinage. - Essences et climat - Difficultés. - Forêts de défense........ 151 à 158

TIRE ET AIRE. - Inconvénients de la méthode. - Son abandon

Coupes a Blanc-ÉToc. - Défrichement, culture et repeuplements artificiels . . . . . . . . 159 à 160

Gemmage. - Quarres. - Gemmage à mort. - Produits résineux. 160 à 161 


\section{IX. - REPEUPLEMENTS}

Modes de repeuplement. - Semis, plantations, boutures et marcottes. - Comparaison des divers modes de repeuplement . . . . . . . . . 162 à 163 Semis. - Choix des graines. - Récolte et conservation. - Préparation du sol. - Semis en plein, par bandes etparpotets. --Semis sur la neige.-Binages. 163 à 172 Plantations. - Saison favorable. - Extraction des plants. - Précautions contre le soleil et le froid. Mise en jauge. - Pose du plant. - Recepage. 172 à 177 PÉPinières. - Choix de l'emplacement. - Première préparation. - Clòture. - Division en plates-bandes et rigoles. - Abris. - Entretien. - Pépinières volantes . . . . . . . . . . 177 à 182

Boutures. - Essences propres au bouturage. - Modes d'exécution. - Marcottage . . . . . 182 à I 83

\section{X. - TRAVAUX DIVERS}

Écoulement des eaux. - Tracé des fossés. - Régalage des terres. - Fossés de clòture. - Marchepied. - Dimensions. - Sources. - Ravins. - Barrages. - Consolidation des berges . . . . . . . 184 à 188

TAILle des RÉSERves. - Règles principales. - Elagage rez tronc. - Emploi du coaltar. - Branches gourmandes. - Taille des baliveaux . . . . 189 à 190

Viabilité. - Lignes de coupes. - Sentiers inlerdits. Nettoiement des chemins. - Curage des fossés bordiers, etc.

\section{XI. - OPÉRATIONS DES COUPES}

Arpentages. - Arbres de limite. - Dégagement des lignes. - Outils d'abatage. - Jalons, piquets. - Ceinturage des arbres de limite. - Entretien des lignes . . . . . . . . . . 194 à 197

Martelages. - Martelages en réserve, en délivrance. Préparation des virées. - Marteaux. - Empreintes. Criees.

Estrmations. - Marque des arbres abandonnés. -Criées. - Dénombrements. - Cubage des bois équarris, au volume réel, au $5^{\mathrm{e}}$, au 6e, au 1/4. - Tarifs. . 201 à 209 Récolements. - But de l'opération. - Ceinturage des réserves. - Virées. - Griffage. - Appels. - Fausses marques. - Récolement de souches. . 209 à 213 


\section{XII. - DÉGATS DES ANIMAUX}

Les troupeaux. - Bêtes à cornes. - Effets du pàturage dans les bois. - Défensabilité. - Bêtes à laine. - Dévastation des montagnes. - Mise en défense.-Chèvres. - Pores. . . . . . . . . . 214 à 221 LE GIBIER. - Le cerf. - Le daim. - Le chevreuil. - Le sanglier. - Le lapin. - L'écureuil. - Les petits rongeurs . . . . . . . . . . . 221 à 231

Les Carvassiers. - Le loup. - Le renard. - Battues. Appâts empoisonnés. - Fouines, putois et belettes. Chats... . . . . . . . 231 à 234 Les orseaux. - Gibier plume; moyens de le conserver. - Oiseaux de proie. - Utilité des oiseaux nocturnes et des insectivores . . . . . . . . . 234 à 236 LEs insectes. - Les bostriches. - L'hylésine du pin. Le bombyx du pin. - Le hanneton. - La courtilière.

\section{PISGICULTURE}

\section{XIII. - LA PÊCHE ET LES POISSONS}

Le service de la pèche. - Causes de destruction des poissons. - Epoque du frai. - Frayères naturelles. - Frayères artificielles. - Fécondation artificielle. Appareils d'incubation. - L'écrevisse. . . 2/46 à 260

\section{ANNEXES}

Tables de cubage . 307 à 316

\section{DESCRIPTION DES PRINGIPALES ESSENGES FORESTIERES}

Le chène rouvre

Le chène pédonculé . Le hêtre.

Le charme.

L'orme .

Le frêne.

Le sycomore.

L'érable champètre.

Le bouleau.

Le tilleul.

Laune commun.

263
265
267
269
271
273
275
277
279
281
283

Le tremble. Le peuplier blanc Le saule marceau Le sapin L'épicéa.

Le pin sylvestre. Le pin mugho . Le pin maritime. Le pin laricio. Le mélèze . TABLE aLPHABÉtIQUe
285 287 289 291 293 295 297 299 301 303 317 


\section{GUIDE DU FORESTIER}

\section{LES MILIEUX}

CHAPITRE PREMIER

\section{LE GLOBE TERRESTRE}

Forme. - Dimensions. - Refroidissement. - Soulèvements. - Premières couches sédimentaires. - Fossiles. - Chaines de montagnes. - Leur altitude. - Vallées. - Lacs.

La terre paraît avoir été primitivement à l'élat de fusion. Elle a pris, en se refroidissant, la forme sphéroüdale qu'elle conserve aujourd hui, forme due au mourement de rotation dont elle est animée. On sait, en effet, qu'elle tourne sur elle-mème comme une toupie, en faisant un tour entier toutes les vingt-quatre heures. Le globe terrestre a yo millions 
de mètres de tour: mais comme il est légèrement aplati, la distance du centre à la surface, cest-à-dire le rayon, n'est pas le mème pour tous les points. Le rayon le plus grand. celui de léquateur, a 6.3-8.233 mètres; le plus petit, celui du pòle. a 6.356 .558 mètres.

Au moment du passage de l'état fluide à l'état solide, les eaux qui se trouraient réduites en rapeurs se sont condensées et se sont répandues également sur toute la surface du globe. A cette éporque. la terre présentait laspect d'une énorme boule aplatie dans le sens de laxe de rotation. et composée de matières lourdes à l'état de pàte solidifiée à la surface. Les eaux enveloppaient cette boule de toute part. en faisant autour dielle une mer continue de foroo ¿ 5.000 mitres de profondeur. Au-dessus de ces taux, unilormément répandues, sétendait une atmosphire immense, formée des gaz et des rapeurs non encore condensés.

Plus tard, il sest produit dans la masse centrale des mourements probablement analogues a ceux que produisent les rolcans. Sur certains points, la croùte solide a été soulevée au-dessus du niveau de la couche fluide. Il s'est formé la des iles et des continents dont la surface sest ainsi trouréc en contact immédiat aree latmosphire.

Cetle surface, ainsi décourerte, arait l'aspect tour- 
menté des pays de monlagnes; elle élait formée de poches riolemment soulerées et par conséquent disloquées dans tous les sens. Cees roches, hrisées frat les seconsses puissantes qui arairnt déleminé lent soulevement, sécroulerent des hauls sommets dans les crevasses, qui furent les premieres vallees. Les pluies qui commencirent a tomber aussitot apres lapparition des montagnes primitives en haterent la désagrégation. Les eaux sécoulant sur leurs pontes escarpées, entrainerent dans les rallées et de la dans les mers leurs débris réduits a létat de sables et de limons. Ce lut lál'origine des premieres couches sédimentaires. Pendant une longue période de siècles, des soulevements et des affaissements successifs ont, tour à tour, ramené à la lumière les couches qui se formaient au fond des mers, et plongé sous les eaux celles qui avaient d'abord élé soulevées. Ges modifications, antérieures de bien des milliers de siècles à l'apparition de l'homme sur la terre, ne nous sont commes que par les traces qu'elles ont laissées.

Les coquillages fossiles, qui se trourent dans des roches aujourd'hui situées à de grandes hauteur's au milieu des continents, prouvent, d'une manière certaine, que les terrains dans lesquels ils ont été déposés étaient autrefois courerts par les mers. On sait d'ailleur's que le niveau des mers est invariable. 
parce que la quantité d'eau qui existe sur le globe reste toujours la mème. Puisque la mer n’a pu courrir les montagnes et que celles-ci présentent cependant des traces de submersion, il faut bien admettre qüà une époque quelconque les masses rocheuses qui forment ces montagnes étaient sous les eaux et qu'elles ont été soulerées, après aroir englobé dans leur épaisseur les dépouilles des animaux marins dont on y retroure aujourd hui les restiges.

Depuis lépoque où les premiers illots ont paru à la surface des mers jusqü à celle où nous rivons, le globe terrestre a subidenombreux boulerersements. Chacun deux s'est manifesté par la formation de nourelles chaines de montagnes, qui, renant suntercaler dans celles précédemment soulerées, en ont singulièrement modifié la structure. Malgré la confusion résultant de cet encherètrement des rhaines d'époques différentes, on est arrivé à détrepminer à peu près lordre dans lequel ces divers soulèrements se sont produits.

Ces boulerersements successifs ont domme a nos continents leur configuration actuelle; mais cetie configuration a été profondément modifiée par les influences de latmosphère et des eaux.

Les plus hautes montagnes du globe sélèrent a près de 9.000 mètres au-dessus du nireau des mer's. 
C'est dans l'Asie centrale que se trourent ces monts gigantesques. En Europe, le sommet le plus éleve est celui du Mont-Blanc (Alpes), dont laltilude est de 4.815 mètres; le nont Pelvoux, cime la plus élrvée de la chaine des Alpes dauphinoises, ne dépasse pas 3.938 mètres.

Quand on arrive au sommet de ces montagnes, latmosphère se raréfie tellement que l'homme peut à peine y respirer. Aussi ne peut-il habiter ces régions élevées. Il n’y a, sur tout le globe, que quelques points où l'on trouve des habitations situées it une altitude supérieure à 4.000 mètres. Ces habitations sont des maisons de poste et des villages construits dans la chaîne des Andes (Amérique méridionale). En Europe, l'altitude des lieux habités ne dépasse pas 2.075 mètres. Le couvent du SaintGothard, dans les Alpes, est le seul établissement fixe construit à une pareille altitude. Les villages de Saint-Veran (Hautes-Alpes) et le Breuil (Mont-Cervin) sont bâtis à 2.0 qo et 2.007 mètres.

Ces hauteurs qui paraissent si considérables sont en réalité fort petites quand on les compare aux dimensions de la terre. La plus grande profondeur des mers est d'environ 6 kilomètres; la plus grande altitude des montagnes est de 9 kilomètres; il y a donc depuis les points les plus bas du globe jusqu'aux plus élevés une différence de 15 kilomètres. 
Le diamitre moỵen de la terre étant de 12.732 kilomitres, les plus grandes différences de niveau ne représentent que les 11 dix-millièmes de ce chiffre. si l'on roulait figurer sur une boule de 12 mètres de diametre ou de $37^{\mathrm{m}}, 7^{\circ}$ de circonférence, les cavités et les éminences que produisent à la surface du globe terrestre les montagnes et les mers, on ne derrait pas donner aux eavités les plus profondes plus de 5 millimetres. et les éminences qui représenteraient les hauts pics de l'Himalaya nauraient pas 1 centimètre.

La gelée qui désagrege lés roches les plus dures, les glaciers et les avalanches qui entrainent les blors, diminuent peu à peu les hauteurs des montagnes en formant sur leurs rersants de rastes talus déboulement composés des débris des crètes supérieures.

Les pluies qui tombent toujours en plus grande abondance sur les hantes montagnes que dans les plaines, à raison du freid qui rìgne dans les régions éleveses, creusent dans les versants des ravins dautant plus profonds que la eohésion desterrains est moins grante. Ces ravins forment des vallées dans lespuelles d'autres ravins dérersent, avee les eaux, les sables et les galets quielles entrainent. Il sétablii ainsi, surrant la disposition des aretes principales. des canaux découlement qui, se reliant les uns aux 
aulres, ramencul jusqü a la mer les eamx provenaml des montagnes.

Ouand ces canamx, qui soml les puisceanx, les ri-

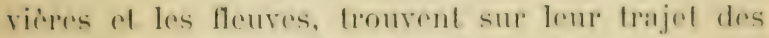
vallées fermées par des barrages nalmrels, il s’y lopme des laes. On refrouve en Franer les fraces de lexistence d'm grand nombre de exs lars aujourd hui disparus. On voit, d laspect de la vallée dont le fond horizontal, formé de dépots d'ean douce, est sourent traverse par un cours dieau, qu ì une certaine époque elle a dù ètre fermée sur um point quin examen attentif permet presque tous jour's de reconnaître.

Les plaines de la Limagne d'Aurergne et du Forez sont les bassins des anciens lacs que formaient l:Allier et la Loire. La plaine d'Alsace a dù ètre recouverte par les eaux du Phin. Si le lit du Rhome s'abaissait de quelques mètres à Genère, le niveau du lac s'abaisserait d'autant et lon rerrait les par. ties aujourdhui couvertes par les eaux paraître au jour, comme on voit apparaitre le fond d'un étang quand on a fait écouler les eaux par la bonde. La plupart de nos plaines sont les fonds des immenses étangs dans lesquels se dérersaient les fleures. Quand le niveau de l'eau a atteint la hauteur de la digue naturelle qui fermait ces étangs, elle a formé sur le point le plus bas de cette digue un 
déversoir par où le trop plein s’est écoulé. Mais peu à peu ce dérersoir a été corrodé, et, à un moment donné, la digue a été emportée par les eaux; alor's le lac s'est vidé et le fleure s'est frayé un lit au milieu des limons, des sables et des galets laissés à découvert.

Ce travail constant de l'eau, qui a déjà adouci des pentes autrefois abruptes, et nivelé des dépressions considérables, se continue de nos jours. Les eaux modifient incessamment la forme de nos continents en entrainant dans les vallées et de là dans les mers les matériaux quelles arrachent aux flanes des montagnes.

Les eaux resteraient confinées dans les dépressions, qui sont les bassins des mers, sil'éraporation ne les ramenait constamment, sous forme de pluies, sur les continents.

Comme les phénomènes de la vie régétale que nous arons à étudier saccomplissent tous dans ces portions du globe ou la croute terrestre est en contact immédiat arec latmosphère et les eaux, il est indispensable de donner quelques notions sommaires sur lair, leau et le sol, milieux dans lesquels naissent, vivent et meurent tous les corps organisés. 


\section{CHAPITRE II}

\section{L'A TMOSPH È R E}

Épaisseur. - Composition. - Oxygìne. - Azote. Acide carbonique. - Carbone. - Origine de l'acide carbonique. - Vapeur d'eau.

On donne le nom d'almosphere à la couche gazeuse qui enveloppe le globe terrestre.

L'épaisseur de cette couche n'est pas inférieure à 80 kilomètres, mais on ne sait pas si elle nest pas beaucoup supérieure. Comme sa densité ra en décroissant à mesure quon sélève au-dessus du sol, on n'est pas parrenu à déterminer la hauteur où les couches supérieures derenues excessivement ténues, et par suite inaccessibles, finissent par disparaitre complètement dans l'espace.

Latmosphère est formée d'air, de vapeur d'eau et dacide carbonique, on y trouve aussi d'autres substances gazeuses, liquides et mèmes solides prorenant des décompositions et des désagrégations qui sopèrent à la surface de la terre, mais ce sont des matières étrangères dont la présence est accidentelle, tandis que l'air, la rapeur d'eau et l'acide carbonique existent simultanément dans toutes les régions explorées de l'atmosphère. 
Lair est un mélange de deux gaz simples, l'oxygène et l'azole.

Loxygine est le gaz vivifiant par excellence; c'est lui qui joue le ròle le plus actif dans les réactions chimiques. Il entretient la combustion et la décomposition des substances organiques et minérales.

Lazote remplit, dans le mélange qui constitue l'air, le ròle de modérateur; il neutralise en partie l'activité trop énergique de loxygène, mais il est par lui-mème impropre à entretenir la vie. Ce gaz entre cependant dans la composition d'un grand nombre de substance qui existent dans les corps organisés.

Liair est formé de 79 parties d'azote et de 21 parties doxygenes (exactement 79,20 et 20,80). La quantité de rapeur deau contenue dans latmosphipe est très variable, en moyenne on léralue at un centième du poids de l'air.

si cette rapeur deau était condensée et répandue uniformément sur toute la surface de la terre, elle y formerait une enche deau de $0^{\mathrm{m}}, 10$ d'epaisseur.

Lacide carbonique, qui est le troisieme dément constitutif de latmosphire. est un gaz composé de 72.73 parties doxygine et de 27.27 parties de carbone. Ces denx substances ne sont pas a l'état de mélange dans lacide carbonique, elles y sont inmbinées de telle sorte que leurs propriétés sont profondément modifiées. 
Le carbone nest autre chose que du charbon rhimiquement pur. Cest un corps simple, soliste, infusible, qui se combine directement aree loxygìne, soms linflueme de la chaleur, et dome ainsi naissance a lacide carbonirque. Le diamant est du rarbone cristallisé. Le charbon de bois est formé de carbone, d'ean et de diverses substances mincrales qui constituent les cendres. Le bois séché à lair renferme 38 pour 100 de son poids de carbone, 35 pour 100 d'eau combinée 25 pour 100 ileau libre et 1 pour 100 de cendres.

Combiné arec dautres substances, le carbone constitue la partie solide de tous les régélaux, il existe sous direrses formes dans les liquides que contiennent leurs tissus. Le sucre, la fécule, les résines sont des hyarocarbures, c'est-a-dire des composés de charbon et d'eau.

La fermentation, la combustion, la respiration des animaux rejettent dans l'atmosphère de grandes quantités de carbone combiné à l'oxygène sous forme dacide carbonique. La décomposition des matières organiques, qui est une combustion lente, est aussi une abondante source de production de ce gaz.

Lacide carbonique est impropre à la combustion et à la respiration. Une bougie allumée plongée dans ce gaz séteint immédiatement. Lhomme et 
les animaux meurent asphyxiés šils respirent l'acide carbonique qui saccumule dans les celliers où fermente la rendange ou dans les puits et les carernes dépourrus daération; lacide carbonique forme, en rolume, les ringt-neuf cent millièmes de l'atmosphère $(0,00029)$.

Quoique ce gaz soit plus lourd que l'air, puisque 100 litres d’acide carbonique pèsent 197 grammes, tandis que le mème rolume dair ne pèse pas tout à fait 130 grammes, il est plus abondant dans lair des hautes montagnes que dans les plaines.

si tout lacide carbonique contenu dans latmosphère était uniformément répandu sur la surface du globe, il y formerait une couche de $2^{m}, 39$, à la température o et à la pression de $0^{\mathrm{m}}, \boldsymbol{7} 6$, ce qui représente en poids 4.765 grammes par mètre carré. Comme l'acide carbonique contient 27,27 pour 100 de carbone, ce dernier corps entre pour $1^{\mathrm{k}}, 30$ dans le poids de la colonne dair qui s'élère au-dessus de chaque mètre carré de la surlace du globe.

La proportion d'acide carbonique de lair que nous avons indiquée comme étant de 29 cent milliemes, est sujette à de faibles rariations. Ainsi dans les grandes villes elle est plus grande que dans les campagnes. Mais les courants dair qui règnent dans toutes les couches de latmosphère produisent une diffusion générale qui rétablit le 
mélange el empiche lacide carbonique de saccumuler dans les couches inférieures.

Lacide carbonirque el partant le rarbone existe en quantites énormes dans les roches calcaires et dans cerlaines roches arcrileuses. Les couches houilleres sont d'immenses dépols de carbone. Ainsi ce corps, qui à létat pur et cristallin est le diamanl, cest-ìtdire la substance la plus prare el la plus précieuse, se trouve en très grande abondance et sous les formes les plus diverses dans toutes les parties du globe terrestre.

Dans les conditions ordinaires, latmosphère renferme un centième de rapeur d'eau, mais cette proportion est très variable. Il y a dautant plus de vapeur d'eau dans l'air que la température est plus élevée.

Lorsque les rents viennent des régions chaudes, en traversant les mers, ils entrainent les vapeurs qui se produisent à la surface de ces grandes masses d'eau. Ces courants, lorsqu'ils arrivent sur les continents, sont chauds et humides.

Au contraire, les vents qui soufflent des contrées septentrionales sont froids et paraissent secs, parce que la vapeur d'eau qu'ils entraînent ne suffit plus à les saturer lorsqu ils séchauffent en arrivant dans les régions plus tempérées.

Les changements de température quéprouvent 
les courants aériens en passant d'une région chaude à une région plus froide ou d'une région froide à une région plus chaude, déterminent, suirant les conditions où ils se produisent, la pluie, la neige, le givre ou les temps clairs, couverts ou orageux.

En France, les rents du sud et de l'ouest. qui ont traversé la Méditerranée ou l'Océan, sont chargés de rapeur d'eau qui se condense et se résout en pluie, quand elle se refroidit en arrirant sur les terres généralement moins chaudes que la surface des mers. Ces rents amènent le plus sourent les temps pluvieux. Les rents du nord et de lest sont au contraire sees et froids, ils amènent habituellement le beau temps. Mais la configuration du sol exerce une influence prédominante sur ces phénomines. Dans les pays montagneux, les rents ne produisent pas les mèmes effets que dans les plaines. Ainsi tel rent, qui dans la plaine a pour effet de rendre le ciel clair et dégagé de nuages, amène, lorsquil atteint la montagne, de la pluie ou de la neige.

La rapeur deau que renferme latmosphire ne devient visible que lorsquelle se contense sous forme de nuage, de brouillard ou de pluie. A létat de rapeur elle est tout a fait transparente. Ciest a sa présence quést dù ce léger tremblotement, quion remarque dans les couches dair les plus rapprochées du sol, pendant les grandes chaleurs. 
La vapeur contenue dans latmosphiere exere une très grande influence sur la régétation, un air trop sec fait flétrir promplement foules les plantes. Elles meurent, si la sécheresse se prolonge.

Les pays ou les pluies sont rares, latmosphiere toujours dépourve de vapeur d'eau, ne peurent nourrir que quelques plantes inférieures, tandis que ceux où il règne une humidité constante ef une température élevée, sont couverts d'une puissante régétation. Mais si la température est basse avec un air saturé de vapeur deau, comme sur les haules montagnes et dans les plaines glacées des contrées boréales, la régétation devient rare et ne se compose plus que darbustes rabougris, de mousses ot de lichens. 


\section{CHAPITRE III}

\section{L'EAU}

Composition de leau. - Hỹdrogène. - Sources, rivières et fleures. - Pluies. - Neige. - Glaciers. - Eaux douces, salines, minérales. - Ammoniaque et acide azotique.

Jusquà la fin du siècle dernier, l'eau a été considérée comme un corps simple, un élément. In savant chimiste, Laroisier, proura en 1789 que ce liquide, prétendu simple, résulte de la combinaison de deux gaz, l'oxygène et l'hydrogène. De nombreuses expériences faites depuis cette époque ont démontré que la combinaison d'un litre d'oxygène arec deux litres dhydrogène donne un litre de vapeur d'eau.

lous arons dit, dans le chapitre précédent. que loxygene est le principe vivifiant de la nature et quil se combine arec le carbone pour former lacide carbonique et un grand nombre dautres corps. L'hydrogène est un gaz comme loxygène et il entre aussi dans la composition d'un grand nombre de substances. Ses combinaisons arec le carbone constituent les résines. les huiles, la cire et beaucoup d'autres matières organiques. 
La densité de lhydrogine est tris faible, ellr est de 0,069 en prenant pour unité celle de laar. 11 price environ quatorze fois et demie moins que ee dernier gaz. C'est cette légiereté qui le rend propre an gonflement des ballons.

Lhydrogène brùle dans l'air avec une flamme pàle. Le résultat de la combustion, qui nest autre chose que la combinaison de l'hydrogène avec loxygène de l'air, est de la rapeur d'eau.

L'eau est la substance la plus abondamment répandue à la surface du globe, où elle existe sous les trois états: gazeux, liquide et solide.

Yous avors vu, dans le chapitre précédent, qu“a l'état de vapeur, l'eau entre environ pour un centième dans le volume de l'atmosphère.

Al'état liquide, elle remplit les mer's qui occupent près des trois quarts de la surface du globe, elle forme sur les continents les réserroirs et les courants qui sont les lacs, les ruisseaux, les rivieres et les fleures. Ces courants ramènent constamment dans la mer les eaux raporisées qui s'en échappent pour venir se déverser en pluie ou en neige sur les continents.

Les sources sont les points d'affleurement des courants deau qui circulent dans le sol. Elles sont produites par linfiltration des eaux pluviales à travers les couches superficielles de la terre. 
Les sources, qui donnent naissance aux ruisseaux et par suite aux rivieres, aux lacs et aux fleures, sont alimentées par les pluies et les neiges, c est-adire par le produit de la condensation de la rapeur d'eau que contient l'atmosphère.

Le phénomène de la formation de la pluie s'explique de la manière suivante :

Yous arons dit que lair contient toujours de la vapeur deau et qüil en peut contenir d'autant plus qüil est plus chaud. On sait d’ailleurs quen séchaulfant lair se dilate, devient plus léger et par conséquent disposé à sélever.

Or. lorsfu un courant d'air chaud saturé de vapenr d'eau, comme le sont les rents du sud et de l'ouest. arrive sur nos ciotes dont la température est plus basse, il śélive dans latmosphere, dont les couches supérieures sont toujours froides, il s'y dilate paree quil supporte une pression plus faible et ne peut conserver à létat de vapeur l'eau qüil contient. Cette rapeur quil abandome se condense et forme des nuages qui se résolrent en pluie.

Il se produit là un phénomène pareil à celui quon peut observer tous les jours au passage diun train de chemin de fer'.

La rapeur séchappant de la machine sélève rapidement dans lair. A sa sortie elle est transparente, a peine visible, mais à quelques mitres au-dessus 
de la locomolive elle se refroidil, se condense of forme une longue traine muageuse. Ce nuage se dissipe hentot dans lanr, mais par les temps foroble il sen échappe une pluie fine dont on voil tres bion les traces sur la voie.

Comme les hautes rigions de latmosphipe sont toujours très froides, les masses de rapenr tiriles entrainées par les rents chauds sont bientôt condensées et forment les goutteleftes qui constituent la pluie. Si le refroidissencut est tres vif, la vapent est immédiatement transformée en neige.

Les rents du nord et de l'est se dirigeant reps des régions plus chaudes donnent lieu d̀ des phénonènes différents. Comme lair dont ils sont formés est froid et partant plus dense que celui des contrées traversées, il ne tend pas à sélerer. Son pouroir dissolrant de la rapeur d'eau augmente d̀ mesure qu il s'échauffe, cette rapeur reste donc à létat gazeux, il ny a pas condensation, pas de nuages, et le soleil garde tout son éclat. On dit alors quil fait beau temps.

Leau se présente a l'état solide sous la forme de grace ou de neige. La neige qui se produit par le refroidissement subit de la rapeur deau contenue dans l'air est formée de très fines aiguilles de glace agglomérées.

La glace est de leau solidifiée par le froid. Dans 
les régions polaires, où le froid est très intense, la surface des mers est presque toujours couverte de bancs de glace qui forment une barrière que l'on nomme banquise, d’immenses glaçons se détachent de temps en temps de la banquise et flottent sur les eaux, jusquà ce qu’entrainés par les courants ils riennent se fondre dans des mers plus chaudes.

Chez nous la mer ne gèle jamais ; mais il nest pas rare de roir les ruisseaux, les rivières et mème les fleuves se courrir de glaçons qui sont quelquefois assez nombreux pour se souder entre eux et former une croùte solide au-dessus des eaux.

La neige, qui tombe en abondance sur les hautes montagnes, les courre d'une couche épaisse que la chaleur de l'été est insuffisante à faire fondrè. Ce sont les neiges éternelles. Quand ces couches de neige saccumulent sur des rersants rapides, clles sont entrainées par leur poids dans les hautes vallées, où elles s'entassent et forment les glaciers. En été, le soleil fail fondre la surface de ces amas de neige et leau de fusion pénètre à travers leur masse, se congèle en agglutinant les petits cristaux de neige et finit par leur donner la consistance de la glace compacte. Toute cette neige fondue nest pas transformée en glace. Une partie trarerse les fissures du glacier et séchaple à leur base en formant des 
reviennent dans les mers en suirant les pentes des continents.

Les eaux de la mer sont salées, celles des sources sont douces. En se raporisant. leau subit une véritable distillation. Les sels quielle renferme restent dans la mer et la condensation des rapeurs donne une eau parfaitement pure. Mais en traversant latmosphère, cette eau dissout une certaine quantité d'air, d'acide carbonique el de quelques autres gaz. elle entraine des poussieres; aussi quand elle tombe sur le sol en neige ou en pluie, n'est-elle pas absolument pure.

L'eau des sources et des rivieres est moins pure que celle des pluies; car elle renferme toutes les substances solubles contenues dans les terrains quelle a traversís. Quand ces substances sont en faible proportion, leau est dite douce ou polable et peut etre employée aux usages domestiques. Si la proportion des matieres dissoutes est assez forte pour donner à leau un goùt spécial et despropriétés industrielles ou médicinales, elle rentre dans la cattégorie des eaux salines ou minérales. Leau est le grand dissolvant de la nature. C̈est un élément nécessaire a la vie de tous les etres organisés. Elle entre dans la composition de toutes les parties des régétaux. Ciest elle qui dissout et charrie dans leurs tissus les matériaux destinés à ies faire croître. Cest 


\section{F $\triangle$ U}

par leau que les sels quidles conlirml pémidrent jusque dans les organes interienrs des plantes, oil on les relrouve sous forme de cendres.

Les eaux pluviales renlerment foules des gaz quielles ont absorbes en traversant latmosphire. Ces gaz sont un air contenant plus doxygine que lair atmosphérique, une petite quantiléd'acide carbonique el quelques traces des corpsqui se forment par la combinaison de lazole, de loxygine ef de la vapeur d'eau. Ces corps sont l'acide azolique ef l'ammoniaque, ils sont en tres faible proportion daus l'atmosphère puisqu ils ne reprósentent que denxeent millionièmes de son poids, ils paraissent cepenulant exercer une influence active tris sensible sur la végétation.

Lair, l’oxygène etl'acide carbonique en dissolution dans l'eau fayorisent en effet les réactions et les combinaisons chimiques qui sopèrent dans le sol et dans l'intérieur des végétaux.

On constate l'utilité de laúration des eaux en comparant les résultats obtenus par les irrigations faites avec l'eau des puits, qui est peu aérée, avec celles quion fait arec des eaux courantes, qui sont d'autant plus chargées d'oxygène qu'elles ont été plus battues dans leur parcours. 


\section{CHAPITRE IV}

\section{LE SOL}

Sols arables. -. Sols d'alluvion et de désagrégation. -

Roches ignées, sédimentaires, métamorphiques. -

Granits, talcs et quartz. - Calcaires, grès, argiles. Humus. - Définitions. - Effets de la culture,

On nomme sol la couche supérieure de la croùte solide du globe terrestre. Le sol est formé de matieres minérales plus ou moins consistantes, désignées sous le nom général de roches, quoique beaucoup dentre elles soient loin d'aroir la solidité et la compacité de's roches proprement dites. Ainsi, dans le langage scientifique, les sables, les argiles sont qualifiés de roches, aussi bien que les granits et les marbres.

La partie superficielle du sol, qui est en contact immédiat avec latmosphère et dans laquelle croissent les régétaux, constitue le sol arable ou propre a la culture. Cette couche, dont l'épaisseur est très variable, est composée des détritus des roches désagrégées et des matières organiques préexistantes.

Les sols formés de matières entrainées par les eaux et déposies par elles, comme les sables et les 
limons, sont appelés sols d'alluvion. Ceux qui résultent de la décomposition sur place des rochess sous-jacentes, qui se délitent au contact de l'air, sont dits de désagrégalion.

Il est fort utile de savoir distinguer les diffirentes espèces de roches, afin de pouroir apprécier les propriétés des sols qu'elles ont contribué à former, car chacune d'elles donne un caractère particulier aux sols dans lesquels ses éléments dominent.

Les roches, qui ont subi une fusion complete par laction des feux souterrains, sont appelées roches iynées. Celles qui sont formées des matières déposées par les eaux sont dites sédimentaires. Il y a, en outre, une catégorie intermédiaire qu’on désigne sous le nom de roches mélamorphiques. Ce sont des couches sédimentaires, situées dans le roisinage des matières incandescentes, qui ont formé, en se solidifiant, les roches ignées. La cuisson produite par ces masses mises en fusion à une température très élevée a donné, aux couches sédimentaires qu'elles ont soulerées et souvent recouvertes, une texture peu différente de celle des roches ignées.

Les roches ignées prennent, suivant leur composition, les noms de porphyres, granits, trachytes, basaltes, talcs, quartz. Les porphyres, les granits, les trachytes et les basaltes sont en grande partie composés de feldspath, matière dure à l'état cristallin, 
mais susceptible de s'altérer et de se réduire en argile. Les granits sont des porphỵres dans la pâte desrfucls il entre des grains de quartz et de mica. Ces poches feldspathiques se décomposent plus ou moins facilement au contact de lair. Les sols prorenant de leur désagrégation sont graveleux. peu consistants et peu fertiles.

Les trachytes et toutes les roches dorigine volcanique, qui subissent une décomposition profonde. produisent, au contraire, des sols d'une grande lertilité.

Les massifs montagneux de la France centrale et de la Bretagne sont en grandr partic formés de terrains granitiques. Ces terrains sont maigres et plus propres à la culture des bois qüa celle des céréales. On y cultive cependant le seigle. le sarrasin et la pomme de terre. Les prairies naturelles y sont nomlreuses, mais elles ne donnent pas un fourrage tris nutritif:

Les roches talyueuses et micaceses ont souvent la consistance schisléuse, cest-à-dire quelles sont formies de minces feuillets superpunsis. Cies roches so: délitent aisément, les feuillets se sipparent, se Jrisent et forment un sol léger. sans consistance ef d'ume lertilite tries mélinere. Les roches quartzensessonl généralement dures et compactes: la silice est la base de leur composition. Les terrains où domine 
cette substance sont tres legers, tries perméables of d'une très laaible fertilité.

Le's roches sédimentairessont les calcaires, les grers, les argiles et les marnes.

Le calcaire ou carbonate de chaux est la roche qui constilue la plus grande partie des enuches s:upérieures du globe. Les terrainsealcaires forment les deux tiers du territoire de la France. Les Alpes, les Pyrénées, les montagnes du Jura, les coteaux de la Bourgogne, ecux de la Guyenne sont composés de roches calcaires. La craie, qui affleure dans les plaines de la Champagne et de l'Artois, est un calcaire.

Cette dernière roche, qui est du carbonate de chaux presque pur, produit des terrains très légers, qui redoutent beaucoup la sécheresse. Quand la couche réduite à l'état terreux est mince, elle est presque infertile. Arec un peu de profondeur et d'humidité, les terrains crayeux deviennent assez fertiles, šils sont suffisamment fumés.

Les autres roches calcaires forment des terrains de fertilité rariable suivant la consistance des produits de la désagrégation de la roche fondamentale. Si cette roche est dure, compacte et divisée en gros fragments, le terrain sera sec, maigre, infertile. Si, au contraire, elle se décompose en fragments ténus mélangés d'argile, le terrain sera de bonne qualité. 
Dans la plupart des terres à blé de la France, le calcaire domine.

Les grès sont principalement composés de silice en parcelles très fines, agglutinées par un ciment tantòt siliceux, tantòt calcaire. Ce sont des roches de consistance très variable, friables dans certains pays, ils sont très durs et compacts dans dautres.

Les terrains formés par la désagrégation des grès sont très maigres; ils ne retiennent pas leau. Ce sont des sables fins qui ne deriennent fertiles qu à force d'engrais.

Lorsque les sables de grès sont mélangés de terreau, ils constituent la terre de bruyère, terre légère qui convient à la culture des plantes délicates.

Les argiles proviennent, comme nous lavons dit plus haut, de la décomposition des roches feldspathiques. Elles sont principalement formées d'alumine. Ce sont des matieres douces au toucher, ductiles lorsqu'elles sont humides. Les variétés les pluspures servent a la fabrication de la poterie. Les sols argileux laissent difficilement pénétrer l’air et leau. Quand ils sont imprégnés d'humidité, ils la retiennent fortement. Ils se crerassent en se desséchant ef deviennent tries durs. Ce sont des terrains froids, difficiles à travailler.

L'argile mèlée de calcaire prend le nom de marne. Cette roche a des propriétés intermédiaires entre 
celles de ses componsants. Comme elle se délite aisément, les terrains qu'elle forme sont plus meubles que les argiles et moins légers que les calcaires purs.

Dapris ce qui précede, on roit que les sols de désagrégation ont tous des propriétés différentes, mais quaucun d'eux na celles qui produisent la fertilité. C'est qu'en effet tous ces sols, formés du petit nombre d'éléments qui composent les roches fondamentales, sont ou trop compacts ou trop légers.

Pour quin terrain soit vraiment fertile, il faut que ces éléments divers soient mélangés de telle sorte que leurs défauts se neutralisent. Il faut, en outre, quils renferment une certaine quantité d'humus.

On nomme humus, la substance noirâtre spongieuse qui est le résidu de la lente décomposition des matières organiques. Le terreau quel'on troure dans le tronc des arbres, sous les souches des vieilles bruyères, est de l'humus.

Le carbone qui domine dans la composition de cette substance, ayant déjà fait partie de corps organisés, est très poreux et par conséquent très pénétrable à l'air et à l'eau, dont il absorbe de grandes quantités.

La présence de l'humus dans un sol lui domne toutes les qualités qui produisent la fertilité, car il 
ameublit les terres trop compactes, réchauffe cclles qui sont trop froides, et conserve la fraicheur à celles qui se dessèchent trop facilement.

Les allurions, formées de matières minérales entrainées par les eaux et réduites à l'état de limons, dans lesquels on trouve intimement incorporés le calcaire, la silice, l'argile et l'humus, sont des terrains de première qualité.

On peut dire, d'une manière générale, que la ler. tilité d'un sol est d'autant plus grande qu il est composé de plus d'éléments divers.

Les bouleversements quiont subis les couches supérieures du globe, les modifications quielles ont éprourées par l'effet des eaux, ont produit d'innombrables mélanges entre les matieres qui composent les roches. Aussi la variété des sols est-elle tris grande. Il ny a, pour ainsi dire, pas deux terrains: qui soient identiquement composés des mèmes éléments; mais pour laciliter l'étude de leurs propritifís, on césigne, comme nous larons fait, les sols de désagrégation par le nom de la roche qui y domine. Ainsi, on dit les terrains granitiques, crayeux, argileux, siliceux; quant aux sols composés, ils prennent le nom de la substance dominante, nom auquel on joint celui des roches qui entrent dans le mélangre. Ainsi, on a des sols argilo-calcaires ou calcaireargileux, argilo-siliceux, etc. 
En indiguant ensuite la proportion d'humus qui esl mélangée anx malières minérales, on olutient une définition des sols qui suffit pour indiquer a peu près leur's qualités.

Les substances mincrales qui enfent dans la composition des sols de désagregation se renomvellent conslamment par leffet de la désagregation des roches sous-jacentes; la constitution chimirjue de ces sols est done à peu près fixe en ce qui roncerne ses éléments minéraux, mais il n'en est pas de mème pour ses éléments organiques. L'humus se décompose sous laction de la lumière, de la chaleur et de l'humidité: il subit une combustion lente et se transforme en acide carbonique, qui se répand dans latmosphère. Les sols les plus riches $\mathrm{mn}$ humus peurent donc sappaurrir avec le temps et devenir infertiles lorsquils sont réduits à leurs composants minéraux. Nous voyons sourent ce résultat se produire lorsquion cultive des terres légères, calcaires ou siliceuses, peu prolondes, dans lesquelles il se trouve de grandes quantités d'humus provenant d’anciens bois détruits ou de rieilles bruyères. Les labours répétés ramènent l'humus à la surface et ameublissent le sol; laair le pénètre dans tous les sens, le soleil et la pluie font le reste; au bout de peu d'années, tout lihumus a disparu. Ces terrains, qui araient au début une couleur 
sombre, due à la présence des débris organiques, prennent peu à peu la teinte de la roche dominante, ils perdent bientôt leur fertilité si de fortes fumures ne viennent pas remplacer l'humus détruit.

Cet effet, assez lent lorsquil se produit sur des terres en plaine, s'accélère considérablement pour les terrains en pente. Là les eaux pluviales entraînent, au fur et à mesure de leur formation, tous les composés minéraux solubles, toutes les particules fines, organiques ou autres. Elles ne laissent en place que des matières inertes, et transportent au loin, sous forme de sable et de limon, tout ce qui constitue la richesse du sol.

Les vallées traversées par les grands fleures sont ordinairement formées de dépôts de cette nature. A raison de leur fertilité, ces rallées ont été cultivées les premières. C'est seulement lorsque la population sest accrue de manière à ne pouvoir subsister surcet espace trop restreint quon a commencé a mettre en culture les plateaux plus élerés et les versants des montagnes.

Les forèts qui courraient toute la terre, à l'exception des hautes montagnes, où les grands végétaux ue peurent croître, ont été successirement détruites par l'homme et remplacées par des terres, des prés et des vignes partout où ces cultures ont été praiicables. Mais on ne sen est pas tenu là, et bien sou- 
rent lon a détruit des forets dans des contries oi il y aurait eu tout avantage a les conserver. Ainsi dans les monlagnes on a arraché les bois pour clendre les palurages, ef au lien de bois qui renait tris bien, on a obtenu des herbages dabord assez abondants, mais qui sont derenus si maigres, par la suite, que les moutons peurent à peine s'y nourrir. Ces terrains arrivent peu à peu à un état de complète dénudation. Les eaux y creusent de profonds ravins et entrainent dans les vallées, sous forme de sables et de galets, les débris arrachés aux flancs des montagnes déboisées. Ces désastreuses opérations nont pas été faites dans les montagnes seulement; il est bien des plaines où le terrain peu propre à la culture a été imprudemment défriché. Là encore on a remplacé de belles forèts par de manvaises terres, dont le produit courre à peine les frais de culture.

Laction que le terrain exerce sur la régétation n'est pas seulement due à la nature chimiqne des éléments qui le forment, elie dépend beaucoup de l'état de division de ces éléments et de leur aptitude à absorber l'eau. Le sol qui sert de support aux végétaux peut ètre aussi considéré comme le réserroir dans lequel les racines ront puiser l'eau nécessaire à leur existence. Les infiltrations des courants souterrains, les pluies alimentent ce réserroir où 
leau se trouve maintenue en couches très minces mouillant chaque parcelle de terre.

Pour yu un sol soit propre à la culture des grands régétaux, il faut dabord qu il ait une consistance assez solide; car les racines doirent s'y implanter et śy fixer de manière à assurer la stabilité du végétal.

Il faut, en outre, quill soit perméable à laair, et quil conserve toujours une certaine quantité d'humidité. Ces conditions sont remplies toutes les fois qu'un sol meuble, d'une certaine épaisseur, mélangé d'humus, repose sur des sables, des pierrailles, ou mème sur des roches fissurées, car alors la couche superficielle laisse pénétrer l'eau jusquaux racines qui s̈implantent dans les fissures du sous-sol. Mais si le sous-sol, au lieu dietre pénétrable, est argileux ou formé de roches compactes, leau retenuc par ces couches impénétrables empèche les racines de remplir leurs fonctions; alles pourrissent, et le régétal meurt.

Les terrains tris légers, comme les sables siliceux, se laissent traverser par leau avec une grande facilité ils séchauffent et se refroidissent promplement. Leur surlace se dessiche si vite que les racines des jeunes plants sont brùlées avant davoir pu atteindre les couches plus profondes ou il reste un peu d'humidité. 


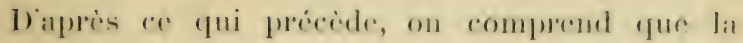
fertilite diun terrain depend non seulement de sat composition, mais encore de sa siluation of de la quantité dean quil renferme. Ainsi, tel ferrain calcaire qui, sur un coteau exposé au soleil, sera de très médiocre qualite, pourra devenir bon dans la vallée oì il sera arrosé par des raux dinfiltration.

Un terrain infertile, quand il est somvent reenuvert par des eaux stagnantes, deviendra excellent quand des travaux de dessichement en auront lait ícouler les eaux surabondantes. En sol de très bonne qualité, mais formant une couche de jeu d'épaisseur sur des roches compactes, pourra ètre tout à fait impropre à la culture forestière, sil est exposé au midi, parce qu il se desséchera trop profondément; exposé au nord, ou rafiaîchi par des eaux d’infiltration, ce mème sol deviendra très bon.

La fertilité dépend donc d'un grand nombre de causes dont la détermination scientifique serait fort compliquée, si l’on roulait établir un classement exact de la raleur relative des divers sols. Il niest heureusement pas besoin de recherches aussi complexes pour apprécier à peu près la qualité d'un terrain domné. La couleur, l'aspect du sol, sa profondeur, et surtout l'apparence des végétaux qui y 
croissent, fournissent à cet égard des indications assez sûres pour dispenser les forestiers des analỵses chimiques que les agriculteurs sont obligés de faire quand ils reulent se rendre bien compte de laptitude de certains sols à des cultures nourelles. 


\title{
PHYSIOLOGIE
}

\author{
CHA PITRE V
}

\section{NUTRITION}

Germination. - Conditions favorables. - Cellules, fibres et vaisseaux. - Composition dune jeune tige. Racines. - Feuilles. - Boutons. - Circulation de la sève ascendante. - Transformation dans les feuilles. - Assimilation du carbone. - Action de la lumière. - Sère descendante. - Cambium. - Formation des tissus. - Accroissement de la tige et des racines. - Effets du couvert. - Périodes de la vie des arbres. - Jeunesse, maturité, décrépitude et mort naturelle. - Des maladies et de la mort anticipée.

Lorsqu'on place une graine d'arbre, un gland par exemple, dans un sol meuble, humide et abrité par des feuilles contre la vire lumière du soleil, on roit, au bout de quelques jours, si la température est suffisamment élerée, ce gland se gonfler, son enveloppe écailleuse se fendre et laisser passer une petite saillie qui s'allonge et se divise en deux filaments, 
dont liun se dirige dans le sol. tandis que lautre se: redresse du côté de l'atmosphère.

Le premier de ces filaments. celui qui pénètre dans le sol. est le rudiment de la racine, la radicule;

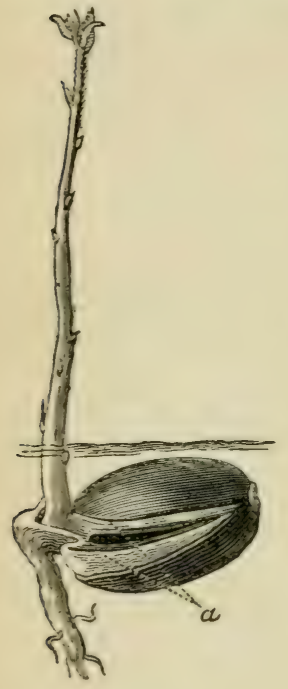

Fig. 2.

le second formera la tige et les leuilles, on le nomme tigelle.

Voici les phénomènes qui se produisent pendant cette phase de l'existence des arbres qui constitue la germination :

Le gland que nous avons pris pour exemple est lormé de deux lobes symétriques accolés par leurs laces et à l'extrémité desquels se troure placé le germe ou embryon. La masse de ces deux lobes, qu'on nomme cotylédons (fig. 2, a), est composée d'une multitude de cellules très petites, juxtaposées les unes contre les autres et remplies d'une substance larineuse quion nomme fécule ou amidon.

Sous linfluenee de la chaleur. le tissu des cotylidons absorle leau qui, comme nous larons dit

Fir. a. - Gland dunt le germe développé a donné nai--ance à la tigelle et it la radicule. 
précedrmmenl, est impregure doxygine empumale a l’ar, ol de sols dissous dans le sol. En présence de ceséléments, la lécule, qui est un composé de cartbone el deau, cide a loxygene ume partie de son carbone, se combine arec ume nourelle quantili: deau et se transforme en me matiere sucréc, laiteuse, soluble, analogue a la sere et capable d'alimenter la jeune plante. Pendant cette période de sa vie, le jeune chène, encore allaché au gland doì il est sorti, se nourrit de sa substance, comme lo petil poulet du jaune de l'reul' ou il est renfermé. Mais bientôt la radicule simplante dans le sol, la tigelle se développe et donne naissance à deux f’uilles qui sétalent au-dessus du sol; les cotylédons épuisés, flétris, devenus inutiles, se détachent et le: petit chène vit de sa vie propre. Il est, à la rérité, d l'état d'ébauche; mais il est complet, car son accroissement ultérieur s'opérera par la seule multiplication des organes dont il est pourvu à ce moment.

C'est ave z ces organes si frèles et d’apparence si simple que la nature saura transformer en un arbre énorme la petite plante produite par le gland.

Toutes les graines qui tombent sur le sol ne germent pas. Beaucoup servent de nourriture aux animaux; il s'en perd un grand nombre qui se décomposent faute d'avoir trouvé, sur le point où elles sont tombées, les conditions nécessaires à leur 
germination. Dans un terrain trop humide, elles pourrissent; elles se dessèchent, au contraire, si l'eau fait défaut. Le froid arrète la germination, une chaleur modérée l’accélère, mais la vive lumière lui est défarorable.

La nature a d’ailleurs pris des précautions contre les nombreuses causes de destruction qui menacent les graines. Elle les a multipliées dans une telle proportion, qu'il suffit de la réussite d'une petite partie d'entre elles pour assurer la conservation de l'espèce.

Si toutes les graines que produit un seul arbre derenaient fertiles, le globe terrestre serait en peru dannées envahi par la postérité de ce seul sujet. Mais cet enrahissement nest pas à craindre, car si les semences sont imnombrables, leurs chances de destruction ne le sont guère moins.

Pour que la radicule puisse sinsinuer dans le sol, afin d’y puiser l'eau nécessaire à l'existence de la jeune plante, il laul que le sol soit meuble et humide. S'il est compact et dur, la radicule, trop tendre pour percer la croùte superficielle, se dessèche el la plante meurt. Elle meurt aussi quand le sol est trop aqueux, parce que l'eau, bouchant tous les interstices du sol, empèche lair d’y pénétrer et d’y apporter loxygène destiné à remplacer celui qui est absorlué. Pendant cette première phase de la régétation, les 
lissus sont pleins de liquides ef par suite tries lendres. Un hale de quelques heures, un comp de soleil, suffisent pour les flétrir. Aussi la nature at-elle pris soin de leur procurer des abris. Ce sont d'abord les feuilles mortes qui courrent le sol, puis le leuillage des arbres. Plus les jeunes plants sont sensibles, plus ces abris naturels sont puissants. On roit toujours, en effet, les arbres à leuillage touffu produire des jeunes plants délicats, tandis que les graines des arbres à feuillage léger donnent naissance à des plants robustes dès leur jeune âge.

Le petit chène, dont nous arons suivi l'érolution jusquau moment où il a commencé à vivre de sá vie propre, est composé de parties bien distinctes, qui sont : la tige, dont le prolongement dans le sol forme la racine; les feuilles, qui s'étalent à l'extrémité de la tige, et le bouton, qui la termine.

Si l'on examine à l'cil nu une section de cette tige naissante, on la roit composée d'une substance homogène, aqueuse, à demi transparente, verte à la circonférence, et d'un blanc verdâtre vers la partie centrale. Mais si l'examen se fait au moyen du microscope, on reconnaît que cette homogénéité n’est qu'apparente, car l'instrument laisse aperceroir, au lieu d'une masse compacte, une multitude de cellules juxtaposées comme les alvéoles d'un gàteau de cire. Chacune de ces cellules est semblable à une petite 
ressie pleine de liquide. Comme elles sont très rapprochées, au lieu de rester rondes, elles saplalissent dans différents sens. Les parois des cellules ne sont pas toujours lisses, elles sont sourent courertes de points ou de lignes déliées ( fig. 3 et 4).

En poursuivant lobservation, on roit que, sur

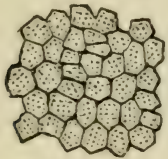

Fiz. 3.
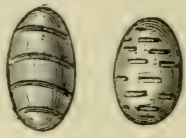

Fis ;.

rertains points, ces cellules sajoutent bout it bout; leurs cloisons intermédiaires disparaissent, et cette suceession de petits globules creux produit des tubes tres fins. auxquels on donne le nom de fibres, lor'sque leur longueur est peu considérable et que leurs parois, dures et épaisses, ne présentent pas d'étranglements. On nomme vaisseaux les tubes forme's par une sirie de cellules ou de fibres soudées bout it bout, et qui présentent des rétrécissements de distance en distance. Les fibres et les raisseaux ont leurs parois lisses ou eriblées de points ou de lignes (fig. 4 ( +5 ). La transformation des cellules en fibres et en raisseaux se lait a mesure que le régétal se développe.

Fig. 3. - Tissu cellulaire grossi.

Fig. 4. - Cellules annelécs, rayées, ponctuées. 
Les fibres se grompont on latseramx disposes en

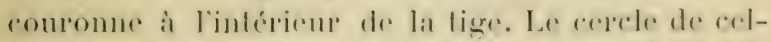
lules qui reste englolus an centre de refle compomme
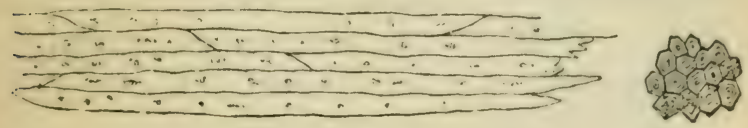

Fix. .).

constitue la moelle. On nomme prayons médullaties les portions du tissu erllulaire placées enter les lanisceaux ef reliaul la moelle à la zone cellubire

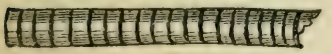

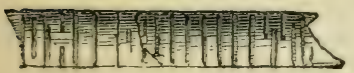

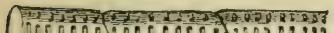

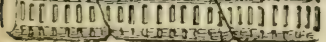

Fi‥ 6.

extérieure, qui deviendra l'écorce. Cette zone est entourée d'un cercle de petites cellules serrées, qui forme l'épiderme. Enfin, cette enveloppe extérieure est elle-mème recouverte par la cuticule, matière homogène semblable à un rernis qui enduit toute la surface du jeune régétal. Lépiderme est marqué de petites taches, dans lesquelles l'examen microscopique lait roir autant d'ourertures qui mettent lintérieur des tissus en communication arec l'atmosphère; ce sont les stomales.

Fig. 5. - Fibres du bois. - Section transversale d'un faiceeau de fibres.

Fig. 6. - Vaisseaux annelés, rayés et ponctués. 
Laspect que présente une section de la tigelle dans

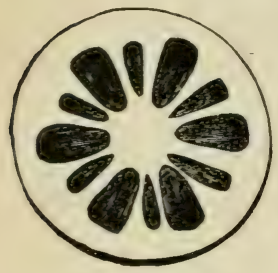

Fig. 7. les premiers jours de son apparition, est indiqué dans la figure 7 .

En examinant quelque temps après une section faite sur une tigelle du même arbre, on voit que son aspect n'est plus le mème. Les faisceaux fibreux, qui formaient une couronne interrompue par de larges rayons médullaires, se sont multipliés; leur cercle, devenu plus complet, est seulement traversé par les rayons médullaires, réduits à l'état de raies plus ou moins larges. Ce cercle de

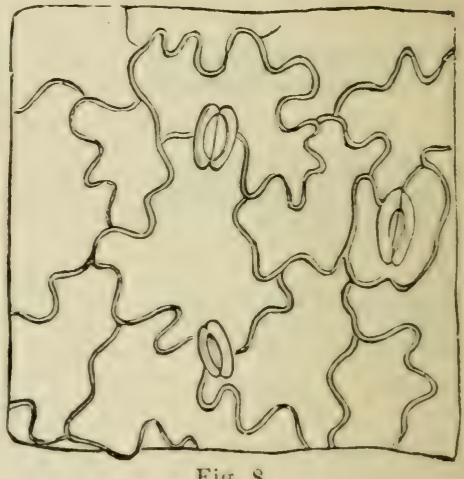

Fig. 7. - Section dune jeune tige. - La partie centrale blanche représente la moelle. qui se relie par les rayons médullaires de mème couleur à la zone corlicale; les faisceaux fibreux sont représentís par les coins émoussés de couleur foncée disposés en couronne autour de la moelle.

Fig. 8. - Portion de lépiderme vue an microscope; les stomates sont figurés par de petits ovales. 
lissu fibreux, qui sépare la moelle de liecorer, formera plus tard le bois (fig. 9).

Arrive à ce point de développement, la lige se compose des partiessuivantes : Au centre, la morlle, formée de cellules laches, grosses ef transparentes dans la partie centrale, plus petites et vertes à mesure qu'elles s'en éloignent; autour de la moelle, un cercle de vaisseaux et de fibres, traversé par les rayons médullaires; qui sont les prolongements de la moelle. Les fibres sont serrées, leurs parois

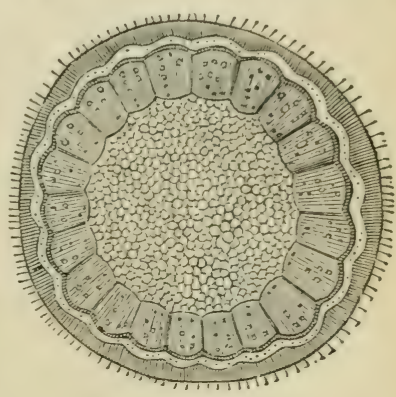

Fin. 9 . sont épaisses et leurs canaux intérieurs napparaissent sur la tranche que comme de petits points; au milieu de ces faisceaux de fibres, on roit des ourer

Fig. 9. - Section diune tige d'érable àgée d'un an. Au centre on roit la moelle entourte diun cercle de faisceaux fibreux. au milieu desquels paraisisent les orifices des vaisicanx. Les limnes noires qui séparent les faisceaux sont les rayons médullaires. Le cambium est figuré par la ligne noire ondulée qui entoure la zone fibreuse. La ligne blanche ponctuée. qui enveloppe le cambium, est formée de fibres corticales et de raisseanx laticiferes. La couche subéreuse, qui entoure les fibres corticales, est recouverte par l'épiderme. figuré par une ligne noire autour de laquelle sont implantés les poils qui courrent la surface de ce tisstt. 
tures plus larges, ce sont celles des raisseaux. Cette couromne de tissu fibreux est entourée dine zone de tissu cellulaire verdàtre communiquant arec la moelle par les rayons médullaires.

Cette zone est le cambium. C'est elle qui sépare

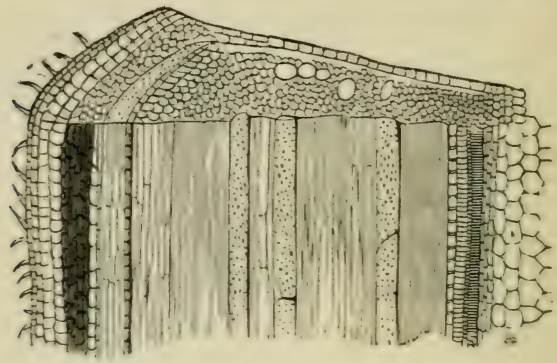

Fig. 10

l'écorce du bois, et cest par elle que sopère laceroissement de la tige en épaisseur.

Le cambium est enveloppé par un cercle de fibres, dont les plus extérieures sont entremelées de raisseaux, ce sont les fibres corticales; ces vaisseaux sont les laticiferes; enfin, autour de ce cercle de fibres, on trouve une zone de tissu cellulaire, coloré en vert, et mis en communication aree la moelle par les rayons médullaires, dont les lames minees trat versent toutes les couches précédentes. Ce'st lenve.

Fig. 10. - Portion de la mème tige coupée dans le sens de -a longueur pour montrer la disposition des dirers tissus. 
loppe collukare, yui est elle-mene recomrele d ume couche de cellules de couleur sourent brume, disposés par assises regulieres ; retle zone, nommón lenveloppe subereuse, est recourerte it son fomr par I'épiderme.

La radicule, à sa naissance, est formée par un petit grompe de cellules, qui, tout en se multipliant, sallongent et se transforment en fibres ef en raisscaux, dont les extrémités supérieures sunissent aux fibres et aux vaisseaux de la tige. Aussi la composition de la racine présente-t-elle une grande analogie arec celle de la tige. La seule différence essentielle consiste dans labsence de moelle; les fibres se déreloppent au centre mème de la racine, et laissent vers la circonférence toute la masse du tisisu cellulaire. L'épiderme des racines n’est pas comme celui des tiges percé de stomates: mais les cellules qui le forment sallongent sourent de maniere à faire saillie, elles présentent alor's laspect de poils très fins.

Le tissu cellulaire des racines est en général gorgé de liquide, et on y constate sourent la présence d'une grande quantité de fécule.

Les feuilles, à leur naissance, présentent l'appar'ence de petites écailles minces et serrées les unes contre les autres; à mesure que la tige s'accroît, ces lamelles, qui sont le prolongement d'un des fais- 
ceaux fibreux dévié de la verticale, sécartent et s'agrandissent. Si l'on examine au microscope une feuille arant pris sa forme définitive, on reconnaît qu’elle est composée des tissus qui se retrouvent dans la tige et quils y sont placés dans le mème ordre.

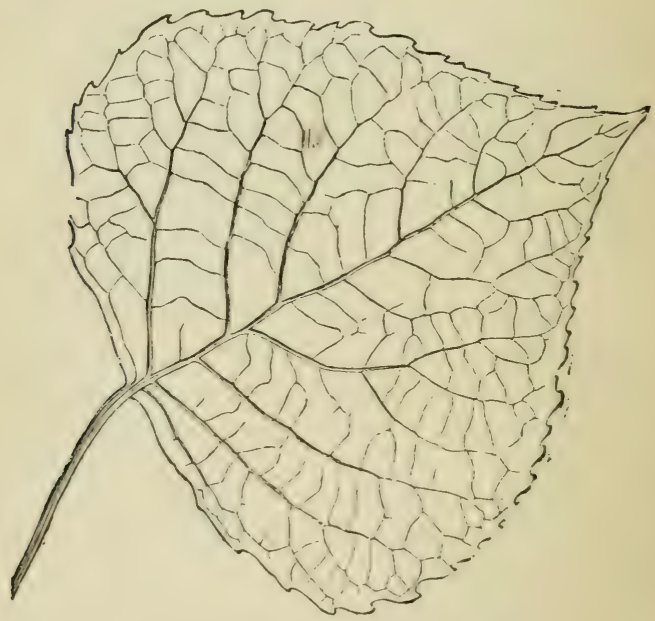

Fin. 11.

Tant que les faisceaux de fibres qui doirent constituer la carcasse de la feuille, restent juxtaposés sur une certaine longueur, ils forment le pétiole ou quene; it une certaine distance de la tige, ces faisceaux se divisent, se ramifient et forment le limbe

Fig. 11. - Feuille de peuplier noir. 
ou la feuille proprement dite. Les mailles du risean que produisent toutes ees petites ramifieations sont remplies de tissu cellulaire.

Quoiquielles soient sourent tris minces, on prut reconnaître dans l’épaisseur des fenilles denx contches distinctes de tissu cellulaire: l'ume supréreme, est formée de cellules serrées, étroites, laissant entre elles quelques lacunes correspondant ì autant de stomates; lautre, inférieure, composée de cellules lâchement unies, présente de nombreuses lacunes, communiquant les unes avec les autres et avec les stomates, qui criblent la face inférieure de la feuille. Les nervures qui viemnent sétaler au milieu de ce tissu de consistance spongieuse sont, comme nous l'arons dit, le prolongement des fibres et des vaisseaux de la tige. Ces faisceaux de petits tubes établissent une communication non interrompue entre la feuille, la tige et la racine.

L'extrémité supérieure de la tigelle est couronnée par le bouton, petit renflement silué entre les points dattache des feuilles terminales; le bouton est le rudiment du bourgeon, qui doit continuer la tige. C'est un amas de cellules, en communication avec l'extrémité de la moelle. Ce noyau cellulaire est recouvert de petites lames superposées, qui deriendront plus tard des feuilles.

Le bouton a beaucoup d'analogie arec l'embryon, 
mais il nest pas comme celui-ci pourru de cotylédons. Destiné à se développer sur place, il trouvera dans le régétal sur lequel il a pris naissance la nourriture que les cotylédons doivent fournir au germe.

On voit.par cette description, que depuislextrémité des racines jusquaux feuilles, toutes les parties du jeme arbre sont mises en communication par une multitude de canaus, qui permettent aux liquides de circuler dans son intérieur. Il nous reste maintenant a faire connaitre comment sopère cette circulation, et quelle est son action dans la vie régétale.

Lorsque, après aroir épuisé les provisions de nouriture renfermées dans le gland, d'où il est sorti. le jeune sujet demande aux milieux qui lentourent les éléments de son existence, sa radicule ra chereher dans le sol leau chargée de substances solubles.

Cette eau est absorbe par lextrémite de la raticule, qui est formée diun tissu cellulaire tris perméahle; les poils déliés, qui gamissent la surlace de cette jeme racine, sont, comme nous lavons dit. des cellules allongres; ils sont par considpuent crenx et augmentent la surface dabsorption. Ces poils. pincitrant dans les plus petits interstices, sappliquent contre les particules terreuses 
qui renterment toujours un peu d'eall a canse de leur consistance spongieuse. Lean absorbée passe de celiule en cellule, a travers les membranes tris lines quiles séparent; elle alleint les fibres et les vaisseaux, gagne le lissu médullaire el monte jusque dans la tige el les feuilles.

Mais pendant ce trajet, cette eau, d'abord presqute pure, dissout dans les cellules de la racine et de la tige les substances nutritives, telles que la fécule, le sucre, qui s'y trourent renfermées, elle les entraine dans soil mourement ascensionucl; à mesure qu'elle s'élève, sa consistance augmente ; ce n'est plus de l'eau, c'est un liquide chargé de substances sucrées ou sirupeuses, qui prend le nom de sève.

La circulation de la sève ascendante s'effectue par toutes les parties du jeune arbre; mais c'est par les raisseaux qu'elle s'opère le plus rapidement, parce que ces longs tubes facilitent beaucoup ses mouvements. On voit toujours la sève se mettre en marche à l’époque où la température s’élève; on est donc fondé à penser que la chaleur est la cause déterminante de cette activité.

Arrivée à l'extrémité de la tige, la sère pénètre dans les feuilles par des vaisseaux et les fibres du pétiole, elle suit les ramifications des nerrures et va se répandre dans les cellules, qui sont, comme 
nous larons dit précédemment, séparées par de nombreuses lacunes mises en communication arec l'extérieur par les stomates.

Il sopère là une modification profonde dans la composition de cette sère, pendant qu'elle circule de cellule en cellule dans lintérieur de la feuille. Au contact de l'air, qui pénètre par les stomates, elle perd une partie de son eau, qui estrejetée dans latmosphère à l'état de rapeur; en mème temps, lacide carbonique contenu dans cet air se décompose sous l'influence de la lumière du soleil, le carbone est absorbé arec un peu doxygène, et le reste de l'oxygène se dégage.

L’air qui sort des stomates est done plus riche en rapeur deau et en oxygine que celui qui entre; par contre, il a perdu lacide carbonique quil contenait. La sève a perdu de son còté une partie de son eau et s'est enrichie des atomes de carbone enlevés à latmosphère, atomes dont lagglomération finit par constituer toute la charpente solide du régétal.

Il est difficile, au premicr abord, de concevoir que lacide carbonique, porté par lair dans les feuilles, puisse produire des quantités de carbone suffisantes pour former de grands arbres. Yous arons dit, en effet, que l'air ne renlerme pas plus de 3 a 6 dix-milliemes de son poids dacide carbo- 
nique, et nous sarons que les cavités intérieures des feuilles sont si petites quon ne peutles voir sans microscope.

Mais, si l'on considire que les feuilles d'un arlore sont très nombreuses, qu'elles baignent dans l'atmosphère, que lagitation du vent fait à chaque instant pénétrer dans leur intérieur de nouvelles quantités d'air, et que chacune de leurs cellules accomplit sans relàche, pendant toute la durée du jour, sa fonction dassimilation, on arrive à comprendre que ce travail incessant puisse, à la longue, donner de grands résultats.

L'atmosphère est d'ailleurs un réservoir inépuisable de carbone; chaque mètre cube d'air n'en contient à la vérité que quelques atomes, mais le rolume de latmosphère est si grand que ces atomes réunis finissent par produire une quantité énorme. Des calculs fort précis, basés sur le poids de l'atmosphère et sur sa composition, permettent d'évaluer à plus de soixante milliards de kilogrammes le poids du carbone qu'il renferme.

La combustion, la décomposition et la fermentation des matières organiques rejettent constamment dans l'atmosphère, sous forme dacide carbonique, le carbone précédemment fixé par les végétaux. Il s'établit ainsi, entre le sol et l'air, un échange constant qui maintient l'équilibre. 
Lassimilation du carbone ne sopère que sous linfluence de la lumière du soleil et par les parties rertes des plantes. Dans l'obscurité, au heu d'absorber le carbone et de rejeter l'oxygène, les rígétaux dégagent de lacide carbonique et absorlent une petite partie de l'oxygine de lair. C'est pour cela quil est si dangereux de laisser, pendant la nuit, des branchages et des fleurs dans les chambres où l'on couche, car l'acide carbonique qui sen dégage est un gaz méphitique.

Cette action de la lumière sur lassimilation fait comprendre pourquoi les plantes maintenues à lombre jaunissent et sétiolent, tandis que celles qui sont exposées aux rayons solaires prennent une belle couleur verte et croissent rigoureusement.

C'est que, dans le premier cas, leffet d'une assimilation lente et incomplète, suite de linsuffisance de la lumière, est détruit par, la déperdition qui se fait pendant la nuit; dans le second cas, au conraire, la déperdition nocturne étant plus laible que lassimilation, la sive conserve sa richesse et donne de la vigueur à la plante.

Il ne laudrait pas conclure de la que les jeunes plants doirent ètre exposés directement aux rayons du soleil. La lumiere est, sans doute, indispensable à tous, mais à des degrés différents. Les plants de 


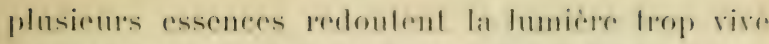
dans leur jeune àge, el rest pour eala quion leme ménage, jusfutau moment oit ils sonl assez forls, des abris qui laissent arriver jusqu it anx ascez de lumiere pour former leurs lissus, fout on intererptant lexees de chaleme qui pourrait les descéchere.

Lorsque la sève a acquis, par son contart areec laar, dans les cellules de la fenille, les qualitios qui la rendent propre à nourrir le régélal, clle rest ramenée dans son intérieur par un mourement contraire à celui qui l’a élevée jusquaux fenilles.

Cette sève élaborée entre dans les canaux formés par les nervures des feuilles, elle arrive au pétiole et pénètre, en suirant le faisceau de fibres qui relie cet organe à la tige, jusquà la zone fibro-vasculaire.

Dans la description de cette zone, il a été dit qu'elle est forméede fibres rayées de petit diamètre, qui constituent le bois, de raisseaux ponctués, dont louverture est plus grande, d'une couche mince de cellules, que recourrent desfibres longues et résistantes, nommées fibres corticales, et de raisseaux laticifères.

Si l'on se rappelle que le pétiole est le prolongement d'un faisceau de ce tissu fibro-rasculaire, prolongement dans lequel chaque partie conserve sa position relative, il est aisé de comprendre qu'en 
s infléchissant, pour s’étaler ensuite sous forme de feuilles, les fibres corticales et les raisseaux laticifères qui occupent la portion externe du faisceau dans la tigre, occuperont la partie inférieure du pétiole et de ses ramifications, et que les fibres formeront la partie supérieure.

La sève dont l'élaboration s'est faite dans les cellules des feuilles troure là des canaux tout préparés pour la ramener. Ce sont ceux qui forment le prolongement des raisseaux laticifères et des fibres corticales; elle s'y engage et descend ainsi dans cette partie de la tige qui constitue la couche intérieure de l'écorce, et quion nomme liber, à cause de sa disposition qui rappelle celle des feuillets diun livre.

La sève descendante, qui a acquis la consistance d'un liquide visqueux, se répand entre la couche des fibres corticales et celle des fibres ligneuses; elle sépaissit et donne naissance à une couche composée de cellules en roie de formation, qui sintercale entre le bois et lécorce. On donne le nom de cambium à ce tissu naissant.

Lorganisation du cambium, dabord purement cellulaire, se complète peu à peu, les cellules se multiplient; elles se transforment en fibres et en vaisseaux, qui reproduisent exactement les tissus sur lesquels ils sappliquent. La couche du cambium, 
primitirement homogene, se dédouble alors en deux parties distinctes: l'une, composée de fibres et de vaisseanx ligneux, sapplique sur la zone ligneuse préexistante et la recourre; l'autre, formée de fibres corticales, revèt intérieurement la couche corticale et la repousse à l'extérieur.

Le cambium arrive ainsi, en descendant toujours, jusqu'à lextrémité de la racine, dont il accroît la longueur.

La plus grande partie de la sève descendante suit la voie qui vient d'ètre indiquée, mais il en passe aussi une certaine quantité par les fibres du bois et par les cellules des rayons médullaires et de l'écorce. En traversant ces tissus, cette sève épaissit les parois des cellules et des fibres, qu'elle incruste d'une substance nommée cellulose, composée d'eau et de carbone, comme la fécule, mais qui en diffère parce quelle renferme moins d'eau que cette dernière. La sève descendante dépose en outre dans lintérieur des cellules de la fécule, du sucre et direrses autres matières solubles.

Les diverses phases que nous renons de décrire peuvent se résumer ainsi qu'il suit: l'eau, arrivant par les racines, dissout 'dans leurs tissus et dans ceux de la tige les matières nutritives qu'ils contiennent, elle s’élève jusqu'aux boutons, dont elle détermine le développement en bourgeons et en 
feuilles. Lorsque les feuilles sont formées, la sère. qui continue à y arriver, subit dans leur tissu une élaboration qui la transforme en un liquide susceptible d'organisation. Ce liquide donne naissance it une couche de cambium, qui suntercale entre le bois et l'écorce dans toute la longueur de la tige et de la racine. Le cambium se dédouble en deux couches, lune, de tissu ligneux, qui sapplique sur le bois et augmente son épaisseur ; l'autre, de tissu cortical, appliquée à l'intérieur de l'écorce, et qui rejette à l'extérieur les parties précédemment lormées.

La tige saccroit donc d'une couche de fibres, qui se dépose sur le tissu ligneux comme une couche de peinture sur une baguette. Lépaisseur de cette couche dépend de la vigueur de la végétation, et varie suirant les essences et la température. L'écorce, au contraire, saccroît en dedans, comme un tube quion enduirait a lintérieur dine couche de vernis.

I la fin de l'été, la leuille qui a accompli sa fonclion perd sa couleur el jaunit, son pétiole se dessèche, elle tombe en laissant roir à son point l'atlache un petit amas de tissu cellulaire, qui est le bouton destiné à donner lieu, au printemps suivant, a ume nouvelle génération de bourgeons; pendant l'automne, les tissus du bois et de l'écorce, impré- 
gnés des substances apportées par la sève descendante, prennent de la consistance, les racines contimuent à croître par leur extrémité, mais ce travail fout intérieur ne se manifeste pas au dehors. Les froids de l'hiver arrètent complètement la régétation.

Les premières chaleurs du printemps produisent sur le jeune plant, dont nous renons de suive le développement, un effet analogue à celui quelles ont produit l’année précédente sur le germe d’où il est sorti. Les boutons, situés à la base des feuilles et au sommet de la tige, s'accroissent comme s"était accru le germe; mais au lieu de tirer, conme ce dernier, leur nourriture des cotylédons, qui ont disparu, ils la trourent dans les provisions déposées par la sève descendante dans les tișsus de la tige.

L'eau que les racines tirent du sol monte dans la tige en se chargeant, pendant son trajet, de fécule et de sucre ; elle arrive aux boutons, qui grossissent, s'allongent en bourgeons et donnent naissance à une nouvelle production de feuilles.

Les phénomènes que nous arons déjà décrits se reproduisant tous les ans, dans le même ordre, il en résulte : une nourelle couche de tissu ligneux appliquée sur celles qui existaient déjà, une nouvelle couche de fibres corticales s'appliquant à l'intérieur 
de l'écorce, un bourgeon terminal, qui prolonge la tige principale, et des bourgeons latéraux qui forment les branches. La longueur de la tige s'accroît ainsi chaque année de la longueur du bour-

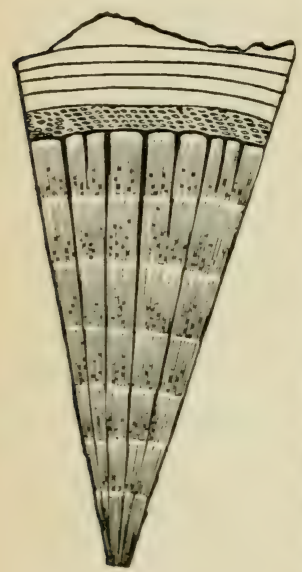

Fig. 12. geon terminal, pendant que sa grosseur s'augmente de l'épaisseur d'une couche ligneuse et d'une couche corticale. Ces différentes couches ligneuses sont assez ỉaciles à distinguer ; dans le chène, elles sont très nettement dessinées par des lignes de points placés sur le bord interne de chacune delles. Ces points sont les ouvertures des vaisseaux qui n existent pas sur le reste de la zone, dont le tissu, formé de fibres serrées, est beaucoup plus compact. Le

bois d'orme présente la mème structure. On ne voit pas ces lignes apparentes de points dans tous les bois; mais dans ceux de nos climats, les couches annuelles se reconnaissent toujours parce que

Fig. 12. - Portion de la tige d'un chène âgé de 7 ans. - Les couches annuelles du bois sont séparées par des lignes blanches. Les couches corticales ne sont pas en nombre égal à celles du bois parce que les plus extérieures, plus anciennement formées, sont déjà détruites. 
le bord externe (còté de l'écorce) est plus dense, plus coloré que le bord interne (coté de la moelle).

On peut done exactement savoir l'âge d'un arbre, en comptant les couches ligneuses de la base du fronc. On pourrait aussi arriver à la mème détermination, en fendant la tige dans sa longueur ; car on derrait retrourer, de cette manière, la série de couches dont on roit les cercles sur la tranche du bois. Mais, comme les fibres vues dans leur longueur sont difficiles à distinguer, la détermination serait moins sûre:

Nous arons vu qu'en mème temps qu'il se forme une couche de bois, il se produit une couche de fibres corticales; on derrait done retrourer dans l'écorce une succession de zones pareilles à celles qu'on voit dans le bois. Cette série de couches corticales existe en effet, mais pendant peu dannées. Le grossissement du bois et l'application successive de couches nouvelles à l'intérieur de l'écorce font que les fibres formées les premières sont peu à peu repoussées à l'extérieur; les couches cellulaires, subéreuses, et l'épiderme qui les recourraient, distendus par cette force intérieure, prennent une apparence rugueuse, s'écaillent et tombent par parcelles ou par larges plaques. Les fibres corticales, -situées immédiatement au-dessous, subissent plus tard le mème sort; de sorte que les couches li- 
gneuses ront en se superposant toujours, tandis que celles de l'écorce se détruisent au dehors au

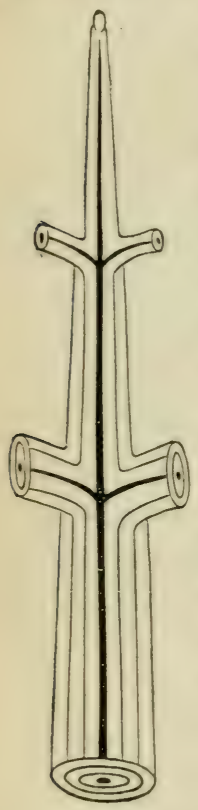

Fig. 13. fur et à mesure quielles se produisent au dedans.

Si l'exposé des lois de la croissance des bois est suffisamment clair. le lecteur peut, dès à présent, en déduire toutes ses conséquences pratiques. Ainsi, il comprendra sans peine comment une empreinte apposée sur un arbre, pourvu quelle atteigne le bois, sera recourerte par toutes les couches ligneuses qui se produiront postérieurement. Si, au contraire, l'empreinte nattaque que lécorce. elle se dilatera comme celle-ci, devien. dra rugueuse, et finira par disparaitre arec les couches sur lesquelles elle a été apposée.

Pour donner une idée aussi nette que possible de la structure diun arbre, nous représentons ci-contre la compe diune tige de trois ans, fendue par son milieu daus toute sa longueur (fig. 13).

Figr. 13. - Tige diun arbre de 3 ans. - Section Inngitudinale Au centre, le canal medullaire que recouvrent les couches annuelles du tissu ligheux. 
On roit que cefle tige est formée de trois troncons, placés bout à bout. Chacun deux est la pousse d'une année. Les hranches latérales, dont on voit la base, sont exactrment composées comme le tronegn né en mème temps quelles, of chacune pent etre considéré comme un nourel axe dou nailront dautres branches, qui domeront a larbre sal forme caractéristique. Si toutes les feuilles devenaient lorigine d'un bourgeon, et si tous les bourgeons se développaient, la ramification suivait une loi très régulière, et tous les arbres de mème espèce seraient identiques; mais larortement des bourgeons réguliers, la naissance de bourgeons adrentifs, modifient cette ramification de manière à la rendre différente pour chaque individu, tout en conservant à tous ceux d’une mème espèce une physionomie particulière, qui constitue le port de l'arbre.

Chaque printemps roit se reproduire les phénomènes que nous arons décrits. La sève s’élève, elle domne naissance à une production de bourgeons; la tige et ses ramifications s'allongent; puis les feuilles naissent, entrent en fonctions, la sève s'élabore, descend et forme de noureaux tissus, qui grossissent la tige et prolongent les racines.

Dans les prenières années, alors que les lissus sont encore jeunes, lascension de la sève se 
fait par la moelle, par les fibres, par les vaisseaux, par tout le régétal en un mot; mais au bout d'une période plus ou moins longue, suivant les essences, tous ces petits canaux s'engorgent, les matériaux charriés par la sève se déposent dans les fibres et rendent leurs cloisons de plus en plus épaisses. La partie centrale de l'arbre se solidifie ainsi peu à peu et finit par former ce cercle de couleur, sourent foncée, quion nomme le cour du bois.

C'est alors par l'aubier, c'est-à-dire par la portion encore jeune, que s'opère l'ascension de la sève, et comme c'est entre l'aubier et lécorce que s'applique chaque année la couche nouvelle de cambium, le cour ne participe plus à la vie de l'arbre, laquelle se troure tout entière reportée à sa circonférence.

Cette partie centrale, devenue inactive, n'a plus d'autre destination que de soutenir le végétal ; c'est un support sur lequel viennent s`appuyer les générations successives de bourgeons, support qui peut disparaître en partie sans entrainer leur mort. On voit, en effet, des arbres vivre, pendant des siècles, alors que leur tronc est entièrement creux.

Pendant que la tige du jeune arbre, dont nous avons suivi l'érolution, s'accroit en longueur et en grosseur, ses racines s'allongent par la formation constante de nouveaux tissus à leur extrémité. et 
grossissent par lapplication sucersive des conrlurs ligneuses qui continuent celles de lil lige. Lorsque les radicelles ont enlevé an sol ambiant les substances solubles qui mlrent dans lat composition de la sère, elles sallongent pour alleindre un sol nonreau, elles senfoncent pour rechereher daus les conches profondes l'eau dont elles ont un besoin dautant plus énergique que l'arlore enandit davantage. Laceroissement de larbere est dautant plus prompt, ru il a plus de feuilles; aussi la surface des zones concentriques, qui en sont la mesure, ra-t-elle en augmentant avec l'àge.

Au premier aspect, cela semble contredit par l'examen de la section d'un vieil arbre, car on roit sourent les couches les plus extérieures aroir une épaisseur très faible si on les compare aux moyennes. Mais il ne faut pas considérer seulement lépaisseur de la couche pour en déduire le rolume de laccroissement ; il faut tenir compte de la longueur de cette zone. Or ceite longueur va en augmentant à mesure que la zone séloigne du centre. Ainsi, on peut dire, d’une manière générale, qu’un arbre s'accroìt toujours tant qu il vit. On pourrait ajouter que son accroissement irait toujour's en augmentant, si des influences, que nous allons indiquer, ne venaient 1 arrèter.

Dans les premières années de leur existence, les 
arbres de nos climats ont, en général, une végétation assez lente. Il s'écoule trois ou quatre ans arant quils commencent à croitre avec vigueur, mais lor'sqưils ont passé cette première période sans éprourer daccidents sérieux, ils entrent dans une phase d'activité qui se manifeste par un développement considérable en hauteur.

La force de la régétation se porte principalement ver's le haut de la tige, qui s'augmente chaque année de la longueur du bourgeon terminal. Les branches latérales s'aceroissent aussi, mais comme la sère est appelée plus énergiquement rers le sommet. qui est mieux exposé aux rayons du soleil, leur dérelopfement nest pas aussi actif. Il arrive un moment ou les branches inférieures, privées de laccés de la lumière par celles qui les dominent. sétiolent et meurent. Larbre qui, dans sa jemesse, était ramifir des la base. se dégarnit alor's, el sa tige principale. gui porte le nom de trone. prend la forme diun cylindre plus ou moins irrégulier.

Cefte marche de laceroissement des ardres ent facile a constater dans toutes les expeces de notre climat. mais cest chez les résineux qu elle se manifeste avec le plus de clarté, à cause de la grande régularité de leur ramification.

si lon considere, en effet, un jeune épicia, on le voit prendre, it lisere de $\{$ ou $j$ ans, une forme 
conicque, déterminée par les verticilles de branches, se raceourcissant sucensivement du pied de l'arhpe juscqu'a la base du bourgeon ferminal. Vers an on 30 ans, les branches basses commenernt a sebloler ; unc pousse ammuelle, qui a souvent 1 melpere plus, aceroit chaque ammée la longueme de la lige; a niesure quelle sélève, les branches inférieuress dessichent, tombent, et le tronc se dégarnit. Vers lìge de 60 à 80 ans, l'épicéa a acquis presque foute. sa hauteur; le jet terminal ne saccroìt plus que de quelques centimètres; mais, en revanche, les branches latérales les plus élevées sétalent horizontalement et forment, au sommet diune tige haute de $2 . j$ et 30 mètres, une touffe qui donne aux vienx épicéas un aspect entièrement diférent de celui qu'ils ont dans leur jeunesse.

Ce que nous arons précédenment dit de lartion des rayons solaires donne l'explication de toutes les anomalies que présente la croissance des arbres. Il sulfit, en effet, de se rappeler que lassimilation se fait loujours sous linfluence de la lumière, pour comprendre que le déreloppement de la tige ou des branches doit toujours se produire du còté d'où elle arrive. Quand, par une cause quelconque, laccès de la lumière est intercepté, lassimilation cesse, ct partant l'accroissement.

C'est pour cela que les arbres dominés s'étiolent et 
meurent. C'est encore pour la mème cause que les arbres en massif s'élèrent beaucoup plus haut que ceux qui poussent isolément. Lombrage des lranches supérieures, qui met olsstacle au passage des rayons du soleil, fait dépérir peu à peu les branches basses, et les tiges sont toujours sollicitées à sélerer pour atteindre la couche baignée de lumière.

Dans les arbres isolés, au contraire, la lumière arrive de tous les còtés; les Jranches latérales premnent un grand déreloppement et se courrent d'un feuillage abondant.

Cette ramification puissante empèche la pousse terminale de devenir prépondérante. Aussi roit-on, le plus sourent, les arbres plantés dans ces conditions aroir un trone peu élevé, mais gros, des branches nombreuses, fortes, et une forme arrondie tout à fait différente de celle des arbres de mème espèce renus en massil. Ces derniers, en effet, ont des branches peu rolumineuses, sétalant à lestrémité d’une ligge tris élevée et relatiroment grèle.

Lart forestice repose en partie sur la comnaissance des lois de laccroissement des arbres, il est donc très essenticl de se rendre bien compte des divers organes qui concourent à cette fonction. Les détails dans lesquels nous sommes entré sur lassimilation par les leuilles, la l'ormation successive des 
conches ligneuses, labsorplion par les racines, doivent suffere pour latere comprendre gure, si les racines sont nécessiares pour lournir a la plante lian dont elle a besoin, les fenilles sont indispensables pour puiser dans late le charbon rqui formera les cellules, les fibres el les vaisseaux dont esl composé tout végétal. Il laut done quill y ait, entre les organes dabsorption (rucines) et ceux d'assimilation (feuilles), une corrélation naturelle que lant doit chercher a produire et à conserver, afin dobtenir, dans un espace et dans un temps domnés, la formation de la quantité la plus grande possible de matières ligneuses; ce qui est le but de la culture forestière.

La vie d'un arbre se compose de trois périodes, dont la durée est variable suivant les essences et les conditions locales.

La première, celle de la jeunesse, se manileste at lextérieur par la tendance de la tige à śélerer. Pendant cette période, le feuillage est abondant, l'écorce est lisse et saine, les jeunes pousses sont longues et droites. La deuxième période est celle de la maturité. Pendant sa durée, larbre cesse peu à peu de croître en hauteur, mais son accroissement en grosseur ne subit pas de ralentissement. Les branches prennent un grand développement, le feuillage reste vigoureux, l'écorce est encore saine, 
mais elle derient rugueuse; l'arbre prend une tìte arrondie.

I cetle période succède celle de la décrépitude. rui se termine par la chute de l'arbre. La déerépitude se manifeste dabord par le desséchement de lextrémité supérieure de la tigre. qui ne reçoit plus assez de sire pour continuer à śélerer. Les branches du sommet meurent les unes apres les autres ef tombent, les chients restés adhérents à la tige se déeomposent. Les eaux pluviales, qui coulent le long de la tige el des branches, retenues par ces chicots derenus spongieux, sünfiltrent entre lécoree et le bois, désorganisent les tissus et oreasiomnent res slintements noiratres qui se prolongent jusrua la base du trone.

En mime temps, les eourhes aentrales les plus ancirennes subissent une diromposition lente produite par les reartions rhimiques des substamers dont elles sont formers. Celle décomposition eommener an erentere ef a la base du trone. Lat parlie fuion nomme le ext:p du bois change de couleme.

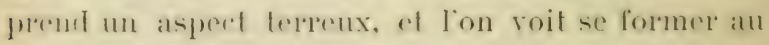
pied de larbere des moredures par lespuelles sorhappe un liquide noiratre. Ces ourertures laissent voir dans lintépieur du trone de grandes caviles pleines d une poussiere brume, qui n'est autre chose que le résidu de la décomposition du tissu ligneux. 
Pendanl que loule apparence de vilalite a cesser

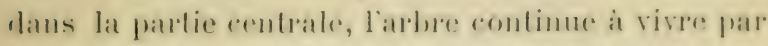
les couches superficielles; sa dure peul idre enreopere fris longue, si les maliomes decompunseres of les caux ont pu tromere nue issure far lat base du frome, paree qualors lintérieur se vide et reste sece si, an contraire, le bois pourri reste enfermé dans be bois sain, leau colrompue dont il est impréené pénetre dans la sere, la décompose el amene promptement la désorganisation de lous les lissus; les branches, nétant plus suffisamment soutenues, tombent alors les unes apris les antres, et entrainent souvent le tronc dans leur chute.

Pourbien se rendre compte des phénomines de la vie et de la mort chez les arbres, il faut regarder

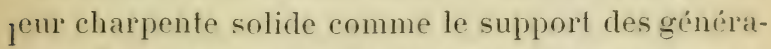
tions de bourgeons qui se succedent depuis la naissance du jeune plant. Chaque génération a la durée de lévolution du bourgeon. Elle commence avec le bouton et finit arec la fenille. Ces générations successires se continuent tant que la tige met les boutons en conimunication arec le sol qui leur fournit leau nécessaire à leur existence. Quand cette communication vient à sinterrompre, l'érolution des bourgeons est arrètée.

On peut done se représenter la charpente d'un arbre comme composée d’une série d’arbres em- 
boités les uns dans les autres, et dont chacun ne vit réellement que pendant la durée de lérolution des bourgeons qui l'ont formé.

Au bout d'un certain temps, les plus anciens de ces arbres, ceux qui sont les plus intérieurs, meurent et se décomposent, les autres continuent à rivre; mais quand la décomposition intérieure a détruit la solidité de la charpente régétale qui les supporte, celle-ci tombe, et cette chute entraine la mort des parties restées virantes.

Les arbres destinés à fournir des bois d'œurre sexploitent pendant leur maturité. Comme la durce de cette période est assez. longue, on peut régler l'ìge de l'exploitation suivant la nature et la rualité des bois quion reut produire. Si lon troure profit à ohtenir de la petite charpente, des pieces longues et elfilíes, on pourra abuttre les arbres dés qu ils cessent de croitre en hauteur. c'est-it-dire au début de la période de leur maturité. Si, au contraire, on veut produire des bois de qualité supérieure ef de fort échantillon, il faudra attendre la fin de cette période.

Dans tous les cas, il est important de ne jamais conserver les arbres au delà de lépoque où ils présentent des signes certains de décrépitude: car ees signes areusent un commencement de désorganisittion intérieure, qui òte aux pièces une grande pantic de leur valeur. 
Il ne faut pas toujours prendre pour un symptims de decrepilude lapparilion de branches mortes vers le sommet dratbes sains dailleurs. Il arrive somsent que les réserves des taillis se enmomment ainsi, sans ètre pour cela dépérissantes.

Lorsque l'exploitation du taillis laisso les liges de ces réserves exposées au soleil, elles se courrent de branches qui absorbent la sive au passage et lem. pèchent darriver en assez grande abondance vers les sommets. Ceux-ci se dessechent alor's, et l'on dit que l'arbre est couromné. Mais si l'on coupe rez-trone ces branches gourmandes, on roit bientit la sìve reprendre son cours habituel, les branches mortes tombent et sont remplacées par de noureaux bourgeons, qui font à l'arbre une nouvelle tète. Il liest mème pas nécessaire de faire cet élagage pour obtenir ce résultat; il se produit tout naturellement par laccroissement du taillis, qui, en sélevant, étouffe les branches basses des arbres de réserve, et fait ainsi refluer vers la tète la force de la végétation.

L'exploitation des bois se fait sourent arant que les arbres soient parrenus à leur maturité. Il est certains produits, comme les écorces, les perches, les cercles, etc., qui ne peurent s'obtenir qu’autant quion abat les arbres pendant leur jeunesse. Quelques essences, le chène entre autres, ne donnent de bon bois de feu que si elles sont exploitées à un àge 
peu avancé. C'est au proprétaire à calculer, daprès les conditions dans lesquelles il est placé, s il a arantage à diriger sa culture forestiere vers la production des bois de gros échantillon, des bois dindustrie ou de ceux de chaulfage. Comme ces conditions sont essentiellement rariables, il est impossible de fixer aucune rigle générale à cet égard.

En traitant de la croissance et de la fin des ardires, uous nous sommes borné a décrire les phases qui précedent et déterminent la fin produite par la vieillesse: mais nous navons pas parlé des causes qui peurent aussi déterminer une mort beaucoup moins lente.

Ces causes peurent agir sur les racines, organes dabsorption, sur les feuilles, organes dassimilation, (.) sur la tige, organe de transmission.

Parmi les causes de nature a entraver les fonctions des racines, il laut plarese en premiere ligne efles qui sont relatives a la quantité ou à la qualité de loan. Le manque deau se manifeste immediatement par le tlétrissement ef lat chute des feuilles: lexces dean détermine une production exagérée de fenilles of de lissus sans consistance. Quand de lean, confrnant des substances rénéneuses, atteint les racines, clle est absorbée et élevée jusiguaux feuilles, qui jaunissent et tombent, larbre meurt empoisonne. Le- matieres grasses, goudronneuses ou sirupeuses 


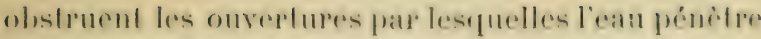

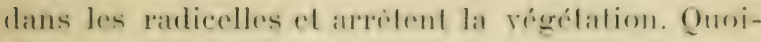
yo il soil asser dillicile de saroir, an premire aborel,

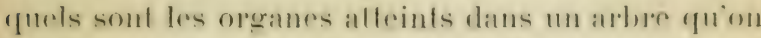
voil deperie, on fent, en général, aflirmer que si

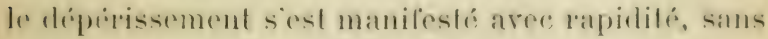
lesion extépiene apparenle, cest vers les rareines qu'il laut en rechercher la cause.

Les forilles, viranl dans latmosphipe, subissont linfluenere de tous les changements gui se pooduisent dans ce milien; lair chaud el see active lévaporation qui se fait a leur surface. La lumirre détermine lassimilation du carbone; rette fonction se ralentit à lombre et cesse pendant la nuit.

Lombrage empèche donc les feuilles de se développer, et comme ce sont elles rui fournissent les matériaux de la tige, on roit toujours les arbres dominés aroir me apparence maladive. Les insectes qui dritruisent les fenilles occasionnent un grand trouble dans la croissance des arbres; rar labsence de leuilles, pendant l'étí, empèche la formation du cambium et entraine par suite une larume dans la série des couches du bois. Si les teuilles ne sont difruites que pendant une saison, larbre peut réparer ce dommage lannée suivante; car la sève ascendante trouvera dans les dernières couches de laubier des matières amylacées encore solubles et 
assimilables; mais si lenlèrement des feuilles se répète pendant plusieurs années consécutires, larbre meurt dinanition. Il est fort important de se rendre bien compte de l'utilité des feuilles; car on suppose trop généralement qu'elles ont dans la régétation un ròle secondaire. Les émanations gazeuses, la fumée et la poussière qui obstrue les pores, le défaut de lumière sont, en dehors des destructions opérées par les insectes. les animaux et l'homme, les causes les plus ordinaires de dépérissement des arbres, par les troubles apportés aux fonctions des feuilles.

Le tronc et les branches subissent les conséquences des perturbations des fonctions des feuilles et des racines, mais ces parties du régétal sont en outre exposées à de nombreux accidents. Les chocs et les blessures qui désorganisent ou tranchent leurs tissus produisent des écoulements de sire dangereux pour lexistence de larbre. Quand les blessures sont franches of quielles nont pas une trop grande surlace. le cambium. qui sérhappe par les raisseanx coupés. forme autour de la plaie $u$ bourrelet dont les bords se rapprochent peu à peu et finissent par la recourrir; mais si la plaie est grancle, le temps que ces bourrelets mettent it se rejoindre est si long. que le bois exposé a lair se décompose arant que la soudure soit faite. Ce bois 
pourri forme alors un centre de décomposition, dont leffet seblend aux parties voisines et finit par entrainer la perte de l'arbre entier.

Certains insectes saltaruent aussi aux liges des arbres, ils se logent sous lécoree, dans les parlies les plus jeunes de laubier et se nourrissent des maticres féculentes et sucrées emmiagasinces dans ces tissus. Les galeries que ces insectes pratiqurut dans le bois laissent échapper la sève, et deviemurnt une cause très active du dépérissement des arlores. 


\section{CHAPITRE V1}

\section{REPRODUこTION}

Boutons. - Bourgeons terminaux, latéraux, adrentifs. Rejets de souche. - Fleur. - Etamines. - Pistils. Fécondation. - Fructifieation. - Direrses formes des graines. - Dissémination.

Les organes de la reproduction des arbres sont les boutons et les fleurs.

Le bouton est dans le principe un petit amas de cellules communiquant arec un rayon médullaire. Caché dabord sous lécorce, il la pousse a l'extérieur en grossissant et se montre sous la forme d'un mamelon courert de petites écailles. éhauches des leuilles qui se développeront plus tard. Le bouton rst un bourgeon a l'état rudimentaire. Il differe de lembryon contenu dans la graine paree que, an lieu d'aroir comme colui-ci une existence entiorement independante, il fait partie diu arbre déja forme, duquel il tire sa nourriture, comme lembryon tire la sienne de ses cotylédons.

Liarbre nest done pas, comme on le pense en général, un ètre unique dont toutes les parties sunt nerestarement dipendantes les unes des autres. C̈.-l un itre multiple presentant une analogie frap- 
pante arec les polypiers qui habitent les mers, car il est forme, comme eux, diune agglomiration d'individus distincto, vivant sur un support commun, mais ayant chacun une existence it peu pros indépendante.

Celle indépendance est assez grande pour quion puisse délacher un des boutons, le porter sur m autre arbre, ce qui constitue la greffe, ou le plant.. dans le sol, ce qui constitue la bouture, ef pourru que lon premue les précautions nécessaires pour que? ce bourgeon soit placé dans des conditions analogues à celles où il se trouvait sur son pied d'origine, il se déreloppera dans sa nourelle siluation, comme il l'eùt fait sur l'arbre d'où il a été détaché.

Chaque bouton est done un individu végétal distinct, croissant sur un support commun, qui est larbre, mais pourant en ètre séfraré.

Ce support sert a methe le bouton en communication avec le sol qui lournit l'eau el les malières minérales nécessaires à son déreloppement. Il sert. en outre, de dépòt de substances féculentes ćlaborées par les leuilles et emmagasinées pour nourrir la génération de boutons qui leur succède.

Il y a trois espèces de boutons: les terminaur. qui sont placés à l'extrémité de la tige dont ils sont destinés à former le prolongement; les latéraux, qui naissent à laisselle de chaque feuille et qui deviendront des branches, et enfin les adventifs, qui 
apparaissent sur des points quelconques de la tige et des racines.

En traitant des fonctions de nutrition, nous arons

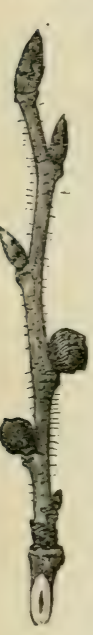
exposé les phénomènes de lérolution des boutons terminaux et latéraux qui déterminent l'accroissement régulier de la tige en hauteur et en grosseur (fig. 1/4); il nous reste à indiquer la nature des boutons adrentifs et les conditions dans les. quelles ils se produisent.

Lorsque la chaleur excite la vie dans les régétaux, leau du sol, absorluéc par leurs racines, dissout, dans son passage ¿ travers les tissus déja formés, les substances quils contiemnent et les rend susceptibles de créer de noureaux tissus. si, au lieu de s'ilever jusquaux boutons, cette sive se trouve arrètée dans son Fi_. $1 \frac{1}{1}$. passage a travers la tige, sur des points dont lactivité vitale est excitée d'une manière anormale. plle sy organise et crée lia un noyau cellulaire qui se developpe a travers l'écoree et apparait à l'extérieur sous forme diune excroissance plus ou moins allongre qui ne tarde pas a se courrir de feuilles.

Fig. 14. - Ramean diorme avee sn houton terminal qui se développera en hourgeon. et les boutons latiraux qui donneront des feuilles et des fleurs. 
Les bourgeons ardentil's different des boutons normaux, non seulement paree qüils noceupent pas, comme ces derniers, une place delepmine dans le végétal, mais encore parce qu ils ne somt pas reenteverls, comme eux, d'écalles destinées à les prómunir contre le froid.

Les bourgeons normaux, formés at la batse des feuilles dès le milien de l’été, doivent, en effet, attendre le printemps suivant pour faire leur évolution; les adrentils au contraire, produils spontanés de la sive printaniere, sc déreloppant dès quils sont formés, nont pas besoin d'ètre protégés contre les rigueurs de l'hiver. On peut artificiellement déterminer la production des bourgeons adrentifs, soit en interceptant le passage de la sìve au moyen de ligatures ou de blessures, soit en actirant la vitalité de certains points de la tige par laction directe des rayons du soleil.

L'abatage diun arbre encore dans la période de la jeunesse a pour conséquence ordinaire lapparition d'un certain nombre de bourgeons adrentifs qui naissent à la base du tronc aux premières chaleur: du printemps. La sève, ne trourant plus sou issue naturelle par la tige et les branches, séchappe en partie par les vaisseaux coupés, mais celle qui est retenue dans la souche y troure un dépôt de matières solubles, dont elle constitue, sous les parties de 
lécorce exposées à la lumière. des groupes de cellules qui se déreloppent en bourgeons adventifs. On donne à ces bourgeons le nom de rejets de souche ou de drageons. suivant quils partent de la base du trone ou des racines.

si, au lieu de couper larbre prés du sol, on laisse au trone une certaine hauteur, les bourgeons adrentif- naissent sur le fùt resté debout. on dit alors que larbre est exploité en tètard. - Les arbres résineux ne produisent ni rejets de souche, ni drageons.

Ces bourgenns adrentils trourent dans la souche sur laquelle ils maisient une ample provision de matériaux nutritifs ; ils recoivent par les nombrenses rarines dont cette souche est munie une srande quantite deau : anssi lenr croissance est-elle tris rapide. Co sont enx qui forment le peuplement des taillis.

Les bourgentrs atrentits dommenl naissance at des ferulles semblables a celles des lonurgenns normaux, et leur diveloppement sieffectue absolument comme celui de ces derniers.

En ontre des houtons normaux et adrentifs, destinés à continuer sur place le sujet qui les porte. lies arbres ont la faculté de produire des graines, rqui domment naissance at des bourgeons semblables a ceux des arbpes dont elles sont issues. mais dont lexistence est mulierement indépendante 
de ces derniers. Les organes an moyen desquels seffectue la produrlion de rees nouveams deres soml les fleurs.

La flem se eompose essemliellement des blamindes, qui sont les organes mâles, c) des pistils, qui sont les organes femelles. Dans les fleur's complètes (fig. 15), le pistil occupe le centre, les élamines sont disposées en cercle autour de lu:i et sont

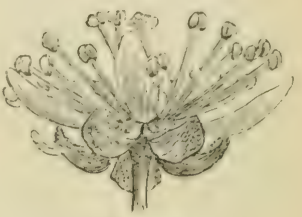

Fin. 1.. elles-mèmes entourées de deux cereles de folioles diversement colorées, qui sont la

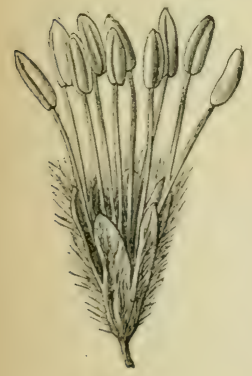

Fig. 16 . corolle et le calice. Quand le calice et la corolle sont ornés de couleur's brillantes, comme on le roit dans un grand nombre de plantes, ils deviennent la partie la plus apparente de la fleur; mais dans la plupart des arbres de nos climats, ces verticilles, qui sont simplement destinés à protéger les organes essentiels, manquent ou prennent peu de développement; aussi le's fleurs des arbres sont-elles en général petites el peu apparentes.

Fig. 15. - Fleur du lilleul à petites feuilles.

Fig. 16. - Fleur mâle du hètre. 
La forme des étamines est différente pour chaque espèce régétale; celles des arbres de nos climats offrent, en général, l'aspect de petites masses de tissu cellulaire, dirisées par une ou plusieurs camne-

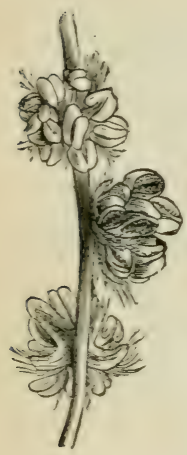

Fig. 17.

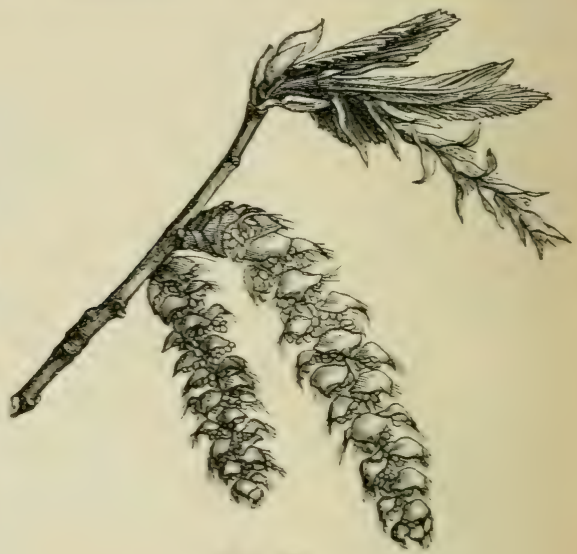

Fir. 1s.

lures et supporties par un peidoncule grile. Ces protites masses sont les antheres. le pédoncule prend le nom de filet. Les étamines du hetre (roy. fige. 16) domment une idée assez exacte de la forme générale di. ces organes. Celles du chène (fig. 1-j), qui ont le filet beaucoup plus court, sont groupées autour de

Fig. 17. - Portion d'un chaton de chène rouvre.

Fig. 18. - Rameau de charme porlant deux chaton- males el un chaton femelle. 
liges grobles el pendintes qui portent le nom dre chalons. On refroure la mime disposition dims les élamines du charme (fig. 18), de l'aune (fig. 19), des pins et des sapins (fig. 20).

Le pistil, organe femelle, comprend l'ovaire, le slyle et le stigmale. L'ovaire est une masse de tissu cellulaire creusée d'une cavité dans laquelle estrenfermé l'orule, qui est le rudiment de la graine. Le style est un tube cylindrique qui continue l'ovaire et dont l'extrémité supérieure s'évase ou se bifurque pour former le stigmate.

La figure 21 représente le

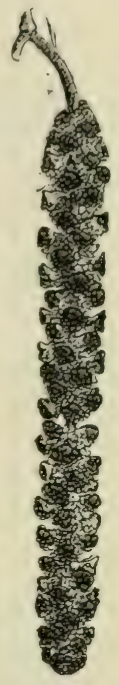

Fig. 19.

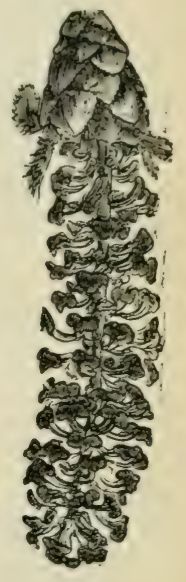

Fig. 20. pistil du frène, la partie renflée inférieure est l’ovaire, le stigmate est situé à l'extrémité supérieure du style, qui le relie à loraire. La figure suirante montre lintérieur de l'ovaire arec $\{$ orules suspendus sur un axe central.

Les étamines et les pistils sont tantòt réunis sur

Fig. 19. - Chaton màle de l'aune.

Fig. 20، - Chaton mâle du sapin: 
une mème fleur comme dans l'orme (fig. 28), le syco-

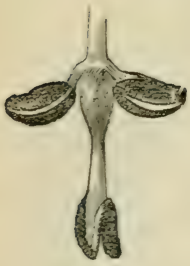

Fig. 21 .

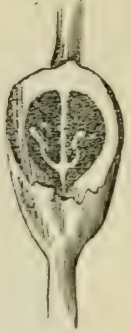

Fig. 22. more (fig. 29), tantôt groupés séparément comme nous l'arons ru pour le hètre, le chène, le charme et l'aune, dont les fleur's mâles ont été figurées plus haut, et dont les figures 23 à 26 représentent les fleurs femelles.

On appelle hermaphrodites les fleurs qui portent des organes males et

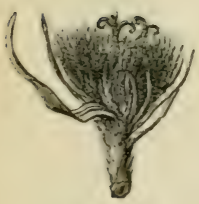

Fig. 23.

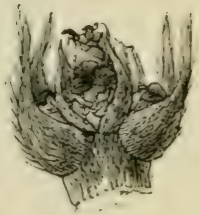

Fir. . . '

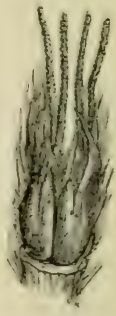

Fir.... .

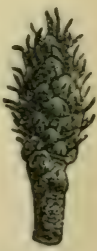

Fir. 26 .

femolles rémis (fig. 28), mailes. celles qui se com-

Figr. 21. - Fleur hermaphrodite du frène.

Fig. 22. - Ovaire du frène, section longitudinale.

Fig. 23. - Fleur femelle du hêtre dans son involucre.

Fig. 24. - Fleur femelle du chêne rouvre.

Fig. 25. - Fleur femelle du charme.

Fig. 26. - Chaton femelle de I'aune. 


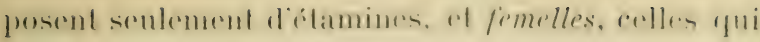
n'ont que des pistils. Les arbres qui portent des fleurs mâlesetdes fleurs femelles sont dils monöques. On appelle dioüyues, ceux chez lesquels les fleur's mâles et les fleurs femelles se trourent sur des individus diflérents.

Il est certains arbres, tels que le frène, qui ont à la fois des fleur's mâles, des fleur's liemelles et des fleur's hermaphrodites. On les nomme polygames.

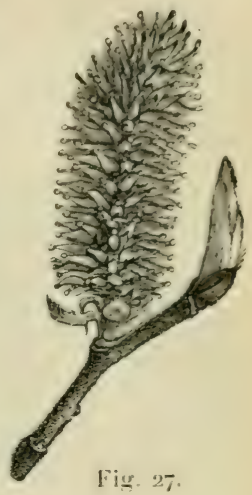

Apres aroir décrit les organes de la génération

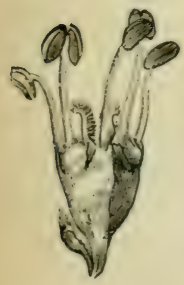

Fi…2.

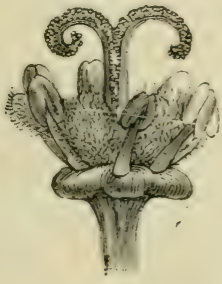

Fi‥ 29. des arbres. nous arons à exposer leur jeu dans l'accomplissement de cette fonction.

Dès que le moment de la fécondation approche, il se creuse dans l'épaisseur du tissu cellulaire dont lan-

Figr. 27. - Rameau à chaton femelle du saule Marceau.

Fig. 28. - Fleur de lorme.

Fig. 29. - Fleur du svcomore dépouillée de son calice et de sa corolle. 
thère est formée, plusieurs carités qui se remplissent d'un fluide mucilagineux dans lequel on roit apparaitre des granulations dontlensemble forme bientôt une poussiere composée de petits grains très fins,

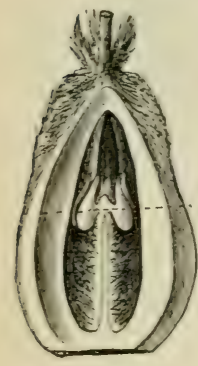

Fig. 30 .

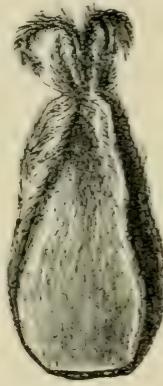

Fig. 31. c'est le pollen. Chacun de ces grains est une cellule microscopique pleine d'un liquide visqueux auquel on donne le nom de foville.

Au moment de la fécondation, les loges des anthères, dans lesquelles est renfermé le pollen, s'ourrent et laissent inhapper cetle poussiere qui va se diposer sur le stigmate des pistils. Lorsutue les pistils et les ćtamines ne sont pas réminis sur la mème fleur, les rents, les insectes transportent le pollen des fleurs mâles sur les fleurs femelles.

Le stigmate est enduit dime maliere visqueuse rui retient les grains de pollen, les fait gonfler et détermine la sortie de la forille quils renferment.

Fig. 30. - Faine portant les pistils flétris.

Fị. 31. - Faine. Section longitudinale montrant les ovules el l'embryon. 
Ce liquide est conduit par les cantux du style jusqu’a l'ovaire, qu'il léconde.

Après la lécondation, il se fait dans la fleur un changement complet. La corolle se lane et se décolore, les étamines et le pistil, devenus inutiles, se dessèchent et tombent, l'ovaire seul se développe, les ovules qu'il renlerme grossissent avec lui et deviennent les graines.

La forme des graines

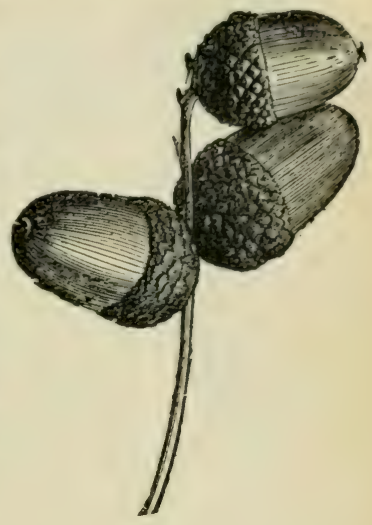

Fig. 32.

rarie suirant les espéces régétales doù elles pro-

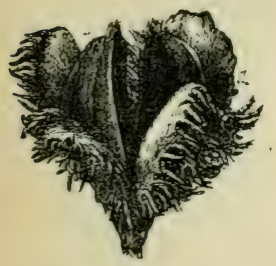

Fig. 33. viennent, mais au milieu des innombrables changements qu'elles subissent, on y retrouve toujours deux parties essentielles : l'embryon, qui donnera naissance à un nouveau sujet, et les cotylédons, qui nourriront cet embryon

Fig. 32. - Glands du chêne pédonculé.

Fig. 33. - Faines enveloppées de leur péricarpe entr'i uvert à la maturité. 


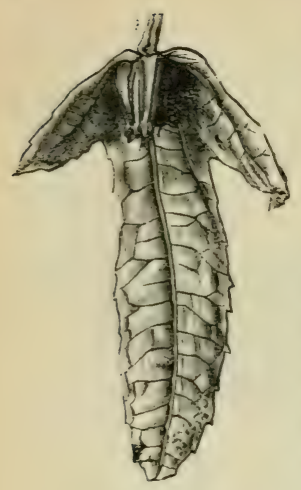

Fir. $33_{4}^{\prime}$.

jusquà ce quil puisse tirer lui-mème sa nourriture de la terre et de l'atmosphère.

La forme des graines dépend du plus ou moins grand accroissement des organes accessoires qui entourent l'embryon. Les transformations de ces organes sont sourent si profondes qu'il derient difficile d'en suivre la trace. On ne roit, en effet, pas beaucoup de ressemblance entre le gland du chine entoure de sal cupule irailleuse (fig. 32), la faîne enfermée dans sa coque hérissée (fig. 33), le fruit du charme avec l'appendice loliacé qui laccompagne (fig. 34) les cònes des résineux (fig. 35) et le chaton qui porte les graines des saules et des peupliers (fig. 36). L'étude attentive du développement de ces graines d'aspect si différent a fait reconnaître que les dissem-

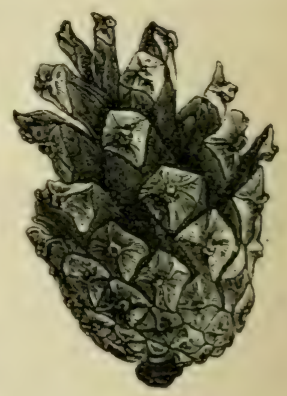

Fig :3.

Fig. 34. - Fruit mùr du charme avec son involucre.

Fig. 35. - Coine du pin stivestre s'ourrant pour laisser échapper les graines. 
blances sont plus ap)parentes que réelles. Les botanistes onl. pu, graice à de patienles recherches, rattacher foutes les formes des graines à quelques types principaux.

Beaucoup d'arbres de nos climats portent des graines munies d'ailes ou d'aigrettes destinées à laciliter leur dissémination.

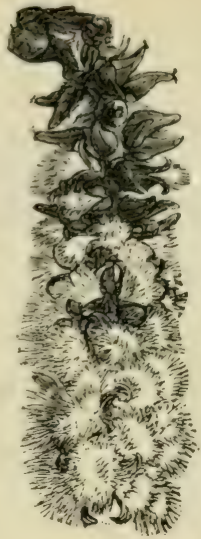

Fig. 3f.

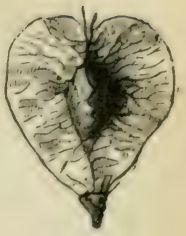

Fiz. : :

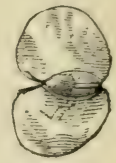

Fir. 35. Les unes, comme les graines de l'orme (fig. 37), sont

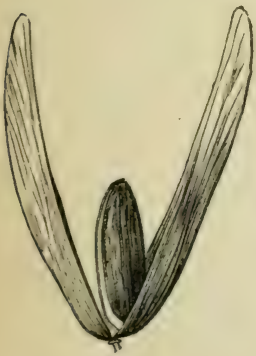

Fig. 39 . placées au centre d’une membrane foliacée; les autres. comme celles du bouleau (fig. 38), du liène (fig. 39), de l'érable champêtre (fig. 4o), de l'érable sycomore (fig. 41), ont deux appendices membraneux. Les graines de la plupart des conif'ères sont aussi garnies d'ailes (fig. /2). Ces appendices per-

Fig. 36. - Fragment d'un chaton fructifere de peuplier tremble. 
mettent aux semences de se transporter à de grandes distances en tournoyant dans les airs, aussi roit-on

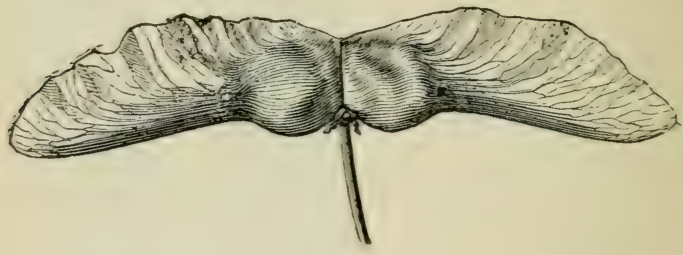

Fig. ¿ $: 0$.

les arbres, dont les graines sont ainsi préparées

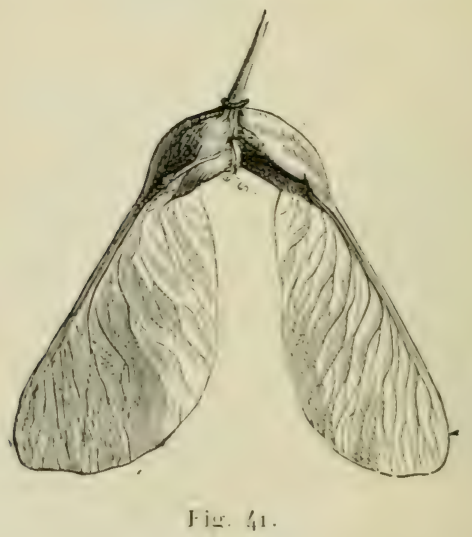

pour de longs royages, se multiplier au loin. Mais il n'en est point qui jouissent sous ce rapport de 
facultés de dissemination plus grandes que les bois biances, salules of peopliers. Lese graines de cers

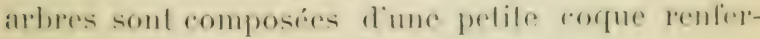
mant l'embryon avec ses cotylédons et surmontées dime aigrelle de poits soyeux (fig. 13). Elles sont disposées en chatons, et leur mulliplicite est tellr. quä lóporque de leur maturité, elles jonchent le sol, qui parait comvert diun duret blane. On comprend quaree de

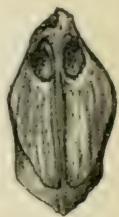

Fig. 42 . tels moyens de multiplication les arbes de cotte espece soient naturellement disposés à curahir toutes les clairicres des bois, et ì empicher par leur nombre le déreloppernent des

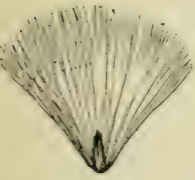

Fig. 43 . essences plus précieuses dont les graines lourdes ne s'écartent pas beancoup de l'arbre qui leui' a domné naissance. Les soins du forestier doivent arrèter cet envahissement et laire predominer dans chaque peuplement les eśénces dont le débit est le plus avantageux.

Tous arons ru que les arbres de nos climats, à lexception de ceux de la famille des conifères, ont la faculté de produire des bourgeons adventifs, et par suite de repousser de souche.

Fig. 43. - Graine du saule Marceau munie de son aigrelte. 
Yous renons de faire roir que tous les arbres qui peuplent nos forèts se reproduisent par les rejets de souche et par leurs semences. Il ne nous reste plus qu“à faire connaître les direr's modes de traitement des forèts, fondés sur ces deux modes de peproduction. 


\section{SYLVIGUL'TURE}

\section{CHAPITRE VII}

\section{T AILLIS}

Traitenext. - Taillis simples el composés. - Réserres. - Essences propres aux taillis. - Sols el climats. Améxagemext. - Durée de la révolution. - Assiette de laménagement. - Division en coupes, immédiate, successive. - Voies de vidange - Exploitatiox. - Abatage. - Cause de dépérissenent des souches. - Nécessité de couper rez-tronc. - Exceptions. - Saison de labatage. - Façonnage. - Vidange. - Résenves. - Importance du balivage - Choix despéserves, baliveaux, modernes, anciens. - Extretiex.-Nettoiements. Restauration des taillis ruinés: par semis de pins et de chènes, par plantations. - Cultunes spéciales. Furetage. - Sartage. - Écorces à tan. - Liège.

Traitement. - On donne le nom de taillis aux: for'èts dont la régénération est fondée sur la faculté que possèdent les arbres de se reproduire au moyen des rejets de souche et des drageons.

Cie mode dexploitation consiste it couper les 
arbres par le pied et à attendre que les rejets aient acquis des dimensions conrenables, pour les couper à leur tour. Linterralle de temps qui sécoule d’une exploitation à lautre sappelle réclulution.

La durée de la révolution est donc égale à làge des rejets au moment où ils doirent ètre abattus. Fixer la rérolution dune forèt traitée en taillis. cest déterminer l'àge où il convient de lexploiter.

Quand on coupe les taillis à blanc-étoc, cest-ìdire sans laisser sur pied aucun sujet, on dit que le taillis est simple. On donne la mème dénomination aux taillis dans lesquels on réserve, it chaque coupe. un certain nombre darbres qui ne seront abattus quä la fin de la deuxième rérolution, cest-à-dire lorsqüils auront un àge double de celui du taillis.

On appelle taillis composés ou sous-fulaie. ceux dans lesquels on réserve, lors des exploitations, des arbres qui doivent ètre maintenus sur pied pendant deux, trois, quatre rérolutions et plus.

Les brins de làge du taillis quion réserve lors des exploitations prennent le nom de baliveaud. A la fin de la seconde rérolution, ils deviennent des modernes. Dans les taillis simples, on exploite toujours les arbres de réserve lorsrưils passent à létat de modernes. Dans les taillis composés, on laisse sur pied un certain nombre de ces modernes, qui prennent à la fin de la troisième révolution le nom de 
coulels. Apres la qualrieme révolution, les ramlels deviemnent des anciens, ef quand ces derniers onl alleint la fin de la cinquiome rérolution, ils passont à l'état de vieilles écorces.

En reserrant dans les taillis quelques arbes de haute tige, on a pour bul dobtenir des bois de fort échantillon en mème temps que des semences deslinées à remplacer les souches qui viement à dépérir. Les arbres ainsi réserrés exercent une influrure très grande sur la régétation du taillis qüils contrent de leur ombre; trop nombreux ou mal espacés, ils peurent arrèter completement la croissance des rejets. Les morts-bois, qui redoutent moins que les autres le manque de lumiere, envahissent alor's le terrain. Il nest pas rare de voir des coupes. daus lesquelles on a marqué beaucoup de réserves jour conserver des arbres de prix, ne produire que de l'ipine noire, des cornouillers et des bois blanes.

Le choix et la distribution desarbres réservés ont une tris grande importance pour l'arenir des peuplements; aussi lopération par laquelle on mareque ces réserves doit-elle toujours ètre faite avec le plus grand soin. Cette opération porte le nom de balivage.

Liexploitation en taillis est un mode tout artificiel, puisqu il nécessite l'abatage des brins à des époques déterminées et Je remplacement des souches mortes. 
sans quoi la forèt passerait à l'état de futaie ou irait en sappaurrissant. Pour qu'elles puissent incléfiniment conserver leur caractère de taillis, les lorits ainsi traitées exigent des soins spéciaux et continus.

Les essences qui repoussent le mieux de souche sont celles qui doirent ètre préférées pour la composition des taillis: parmi celles-ci nous citerons dabord: le chène, dont les souches ont une durée tris grande et une grande force de reproduction. puis le charme, l'orme, le frène et les érables. Nous avons dit que les résineux coupés an pied ne produiscnt pas de rejets; les arbres de cette famille ne sont donc pas propres à ètre traités en taillis. Le hètre repousse de souche dans les bons sols et les climats doux. On trouve en Normandie et dans les montagnes du Midi de la France de superbes taillis de celte essence, mais dans les régions froides de lEst. sur les terrains maigres. les boutons adrentifs rares $1 . t$ pen vigoureux ont beatcoup de peine a perer l'écorce dure des hètres àgés: pour fariliter lessor du bourgeon, il est nécessaire, dans ces contrées. que la enupe soit toujours faite dans le jeune bois.

La faculté de repousser de souche dépend uon senlement de lessence. mais encore de läge dusujet et du lieu de station. Les jeunes arbrescoupés donnent des rejets nombreux et vigoureux. Cette force 
de reprodurtion derent aree l'atge. Elle est moins

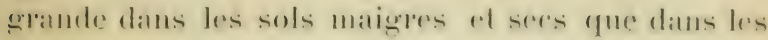
frrains frais of lertiles, dans les regenons lroides fur. dans celles dont le climat est doux.

Il laut eviter de traiter en laillis les forets silures dans des fonds bas ef humides, ou les gelies printanieres sont fréquentes. An moment de leur essor, les rejets de souche sont gorgés deau el patr suite tress sensibles aux changements de lemperafure: lenrs tissus ne se solidifient quapres léte. Suls sont surpris pardes gelées an mois de mai oude juin, moment de leur plus actif déreloppement, ils sont inlailliblement détruits. C"est une pousse perdue. Quand ces accidents se produisent plusieurs fois. le taillis se rabougrit, les cépées s'étalent, beancoup, de souches meurent et il se forme à leur place des clairieres courertes de grandes herbes marécageuses quil devient très difficile de faire disparaitre. Pour maintenir le régime du taillis dans de pareils terrains, il est indispensable de les assainir de maniere ¿ faire écouler les eaux et de faire disparaître arec elles les vapeurs qui occasionnent les gelées. Mais il est, en général, plus lacile de substituer au régime du taillis celui de la futaie. Les bourgeons dépourvus de toute enveloppe protectrice qui viennent sur les souches sont, en effet, bien plus exposés à la gelée que les boutons écailleux des jeunes plants. 
Ceux-ci sont dailleurs moins hàtifs, mieux abrités, ils redoutent donc moins les froids tardifs.

On derra aussi, et par les mèmes motif's, renoncer a traiter en taillis les bois situés à de grandes altitudes, car les changements subits qui se produisent dans la température de ces régions mettent, it chaque exploitation, lexistence du peuplement en danger.

Les terrains frais et profonds, en plaine ou en coteau, sont ceux qui conviemnent le mieux aux taillis; mais, avec des soins convenables, on peut ausi tirer un bon parti de ceux qui croissent sur des sols pen profonds, pourruquils ne soient ni trop secs, ni trop légers.

Les bouquets de bois pen importants sexploitent en une seule fois, quand le taillis a atteint làge où il peut ètre utilisé arantagensement; mais dés yu une forit a une contenance un peu considérable, il est do löntérèt du propriélaire de répartir les coupes de maniere à obtenir, non plus un revenu intermittent, mais des revenus réguliers à peu près égaux : cest ce. quion appelle aménayer une foret.

Aménagement. - Laménagement des taillis consiste di frartager la surface de la forèt en un certain nombre de coupes qui seront exploitées successivement, a mesure que le peuplement aura atteint làge de la rérolution. 


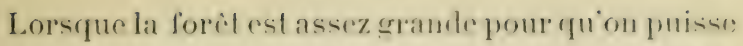
y asseor autant de compes quil ya dimmens dams la révolution, lexploitation sera anmuelle; elle serat biemale, triemnale, si le nomber des compes est la moitié, lé tiers de celui des années de la rérolution. the forit amenagée en taillis présente donc: fonjours me suecession de coupes d’àges gratués. La plus ancienne est garnie de rejets qui ont autant de: pousses que la révolution a diannées, la plus jeume porte le recru de l'année.

La premiere chose à faire quand on reut aménager un bois traité en taillis, cest de fixer la duré de la révolution. La solution de cette question préliminaire dépend: de la nature du sol, du climat, des essences qui dominent dans le peuplement ef surtout des débouchés locaux. Nous indiquerons succinctement les considérations principales d'après lesquelles les propriétaires doivent se guider pour fixer la révolution de leurs bois.

La régénération des taillis étant fondée sur la propriété que possèdent certaines essences de produire des rejets et des drageons, les exploitations doivent ètre dirigées de manière à obtenir, par ce mode de reproduction, une succession régulière et indéfinir des produits les plus considérables et les plus utiles.

Les arbres ne se reproduisent pas indéfiniment au moyen des rejets de souche, aussi ne peut-on assu- 
rer la preptuite des taillis quen remplacant, soit far des repenplements artificiels, soit par des semis naturels prouluits par les arbres réservés, les souches ‘fui riemnent à dépérir. Les rejets, comme les dragrens, ne sont en effot que des tiges nourelles croissant sur les souches ou les racines diun arbre yui a une existence limitép. et dont la vitalité sópuisera dautant plus rite que des exploitations réitérées riendront plus sourent modifier les conditions normales de sa régétation.

La puissance reprodurtive des souches saffaiblit el disparait des que les arbres ont atteint un ige yni varie suivant les essences, le sol et le climat; ditntre part, les rejels produits par des souches exploifis trop sourent sont dépourvus de rigueur : il est done tros important de ne pas attembe pour exploiter les taillis qu'ils soient trop dig is pour se réginérer; il ne lest pas moins de ne pas fatiguer les sjuches par des abatages répétés.

Il y a avantage it exploiter jeunes certains taillis croissant arec rapiditi dans les premieres annés 'fui suirent la coupe. et dont la régétation se andenlit ensulte: il sera avantagemx, an contraire, de relarder lexploitation des bois peuplés desseners dont la régitation, dabord assez lente, ne commenere as setiver quan bout diun certain nombre dannes. Dans les sols fertiles, on peut prolonger la durée 


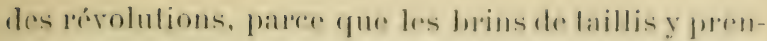
nent whe enrande hauleur. On rapprocherat an contLaire les conpesdans les forids doml le sol est maigre

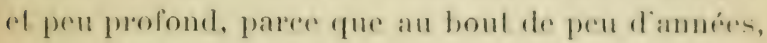

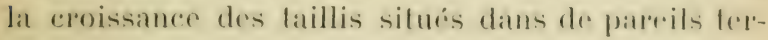
rains se ralentit sensiblement.

Enfin, la durée de la révolution dépend encope de la nature des produits que la forcol est destimé a fomrnir.

On coupe les taillis jemnes quand les bourres, lo menu lagolage, sont d'un débit avanlageux, quand on trouve à utiliser les brins it la confection do corcles, de rotettes, diarticles de vannerie, marchandises qui, lorsqu'elles ont un débouché facile, donnent aux jeunes bois une valeur supérieure à celle quils acruerraient en prenant plus d'accroissement.

On retardera au contraire l'exploitation des taillis qui doirent produire du bois de feu, des perches, de la menue charpente.

Les révolutions généralement adoptées sont pour les essences dures, telles que: chène, charme, hètre, etc., celles de $2 \bar{y}$ à 40 ans, lorsque les bois sont situés dans de bons sols. On ne dépasse pas la limite de fo ans, mais on réduit la rérolution ì 20 ans, et mème au-dessous lorsque le sol est ite qualité médiocre. 
Les rérolutions de 15 à 25 ans sont préférées pour les aunes, bouleaux, trembles et les taillis mélangés d'érahles, de frènes et de fruitiers, tels que sorbier's, merisiers, pommiers.

Enfin on exploite à 8, 10 et 15 ans les taillis de chàtaigniers spécialement traités pour la confection des échalas et des cercles, ceux où dominent les saules. les coudriers, cornouillers et autres mortbois à croissance rapide.

Les rérolutions des taillis simples sont ordinairement courtes; elles ne dépassent guère 20 ans. En général, on napplique ce mode de traitement qu aux torits dont le sol médiocre est peuplé dessences inférienres, et nù les arbres de réserre ne sont pas susceptibles dacupuérir de belles dimensions.

Cependant on exploite anssi en taillis simple le chène, le chaitaignier, le micocoulier croissant dans de bons sols, quand lécoree des jeunes taillis de chène, les cercles fabriqués avec le chàtaignier, les fourches et les manches de fonets confectionnes avec les brins de micosoulier ont un prix élevé qui rendent ce mode dexploitation prélérable à tout autre. Mais le propriétaire, qui ne sabome pas a ces productions spéciales, devra maintenir des réserves dans ses hois. toutes les fois que le sol sera assez profond pour quelles puissent prospérer jusqu à làge où elles dommeront des semences. 
Quand on est fixe sure lat duredede la rewolution el, par eonserfuent, sur le nomber des eompes, il nes peste plus quä les asseoir sur le terrain et i marfuep lemes limiles par des signes fixes. Ceest en qui eromstitue liaménagemenl proprement dit.

Cette operation exige lontervention dim anpenteur, qui lève le plan du bois, en mesure la surline et le partage en coupes dégale contenance. Charue coupe est ensuite assise et délimitée soit par des bornes, soit par des fossés d’angle.

Il ny a pas à faire chaque année larpentage de la coupe à exploiter dans les bois ainsi aménagés, puisque la contenance de toutes les coupes a été calculée en une seule fois.

Les propriétaires, qui ne reulent pas laire, d'un seul coup, les frais d'un aménagement complet, peurent arriver à régler en plusieurs années lexploitation de bois peu étendus, d'une manière suffisamment exacte, en adoptant la marche suirante: ils prendront sur les plans cadastraux un calque du bois à aménager'; ils diriseront la surface totale par le nombre d'années de la rérolution pour obtenir la contenance de la coupe annuelle, puis ils marqueront approximativement, sur le plan extrait du cadastre, la place que doit occuper chacune des coupes, en commençant par les bois les plus âgés. Si la conformation du terrain le permet, ils traceront surce plan 
nue laic sommiere pourant servir de roie de ridange sur laquelle sappuieront toutes les coupes.

O)uand ce croquis de la marche de lexploitation est fait. il ne reste plus qüà le suirre fidelement. Chaque année. lors de larpentage, on asseoira la erupe dans lordre qui hui est assigné. Après l'exphitation on lera ourrir des fossés-bornes, et au bout de la rérolution, on aura une série de coupes bien assises fet dont la contenance sera d̀ peu près igale.

supposons, pour fixer les idées au moỵen diun exemple. que le cadastre ait donne le pourtour du bois dessiné dans la figure ci-jointe (fig.4́), el qüil lui ait assigné une contenance de 1 oo hectares. Si nous romlons amenager ce bois en taillis a la rérolution de 20 ans, chaque enupe derra aroir pour contenance la zoe partic de 120 hechares. soit 6 hectares. Il sagit dasseoir sur le terrain ces 20 coupes. Nous supposons que le bois à aménager oceupe les deux versants dime vallée dont le fond est parcouru par un sentier tortuenx, mais susceptible d'etre transforme en voie de vidange, partout praticable aux roilures rhargés. Tous tratrerons sur le plan mue late sommiere suivant a pen pres la direction du sentier, fuis nous calculerons la contenance de chacune des parties du hois situées a l'Est el a l'Ouest de cette ligne. Supposons que la premiere ait $63^{\text {ha }}, 10 \mathrm{el}$ la seconde. $56^{1 / a}$. 90 : en divisant ces chiffes par celui 
yui reprósente la contenance de la reomper anmmr.lle,

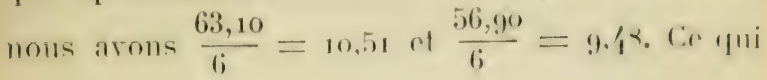

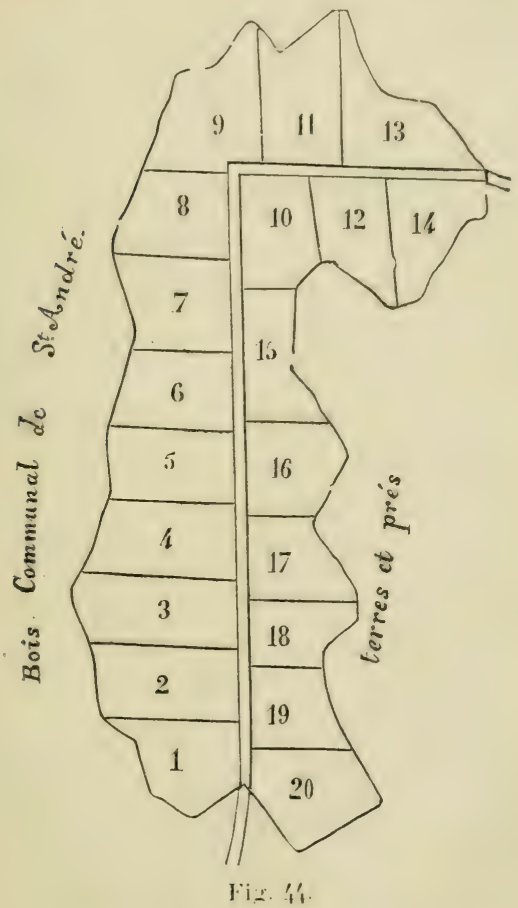

nous indique qu'il y a à l'Est la contenance de 10 roupes plus 0,51 de la contenance d'une coupe, et a louest 9 compes 0, 8 . Ce rui revient à peurle cluse 
près à 11 coupes d'un còté et 9 de l'autre. Divisant done 63.10 par '11, nous aurons la contenance des coupes à asseoir à l'Est de la ligne, et 56.90 divisé par 9 nous donne la contenance des coupes du rersant à l'Ouest. Il ne nous restera plus qu à asseoir charque année la coupe à exploiter daprès le croquis pour arrirer à un aménagement réglé.

Le point capital dans l'aménagement est d'aroir un bon sỵsteme de vidange ; des produits aussi enenmbrants que les bois nont de valeur quautant que les moyens de transport sont économiques. Il faut donc avant tout organiser le plan dexploitation de telle sorte que charque coupe ait une sortie facile sur un chemin praticable.

Les arpenteurs sacrifient sourent cet interet de premier ordre, pour aroir des coupes de forme léguliere ef des lignes sommieres bien droites. Leurs plans sont tris clairs et l'aménagement parait simple mais quand on ra sur le terrain. on roit que ces belles lignes droites escaladent les montagnes et sont impraticables aux voitures. Lesproprictaires yui ne reulent pas sillonner leur bois de perefes inutiles prendront, autant que possible, pour laies sommières les chemins de vidange; et si ces chemins ne peurent serrir de laies sommières, à raisnn de la configuration du terrain, les lignes de division quion établira ne derront avoir que la largeur né- 
ensiaire pour les bien fixer. Il est en effet imulile. deurrir sur 4 ou 5 mitres de largeur des ligures gui liout dautre ntilité que de séparer tes coupes. Les lignes de division des conpes ont ordinairement 1 metre de largeur. Apres chargue exploitation, on releve les bornes, lon cure a vif les sants de chrve qui servent a déterminer diune maniere immuable la direction des lignes, et laménagement par ces soins est indéfiniment conservé.

Exploitation. - La durée des souches et la vigueur dres cépées dépendent en grande partie de la manière dont l'abatage est opéré.

Les bourgeons qui produisent les rejetsse forment sur le pourtour de la souche, entre l'écorce et le bois; si l'écorce est détachée, ils ne peurent naìtre. Il laut done aroir soin de ne pas soulerer l'érore de la souche; pour cela l'abatage doit ètre fait arec des instruments bien tranchants et l'entaille dirigée de bas en haut, car la coupe oblique de hauten bas fait éclater le bois et déchire lécorce. Quelque soin que prenne le bùcheron, il se produit toujours des éclats et des déchirures; pour y remédier, il faut parer la section en retaillant la souche des bords au milieu de manière à lui domner une forme bonbée.

La coupe derra ètre franche et nette pour que leau ne pénètre pas dans les cavités que présenterait une surface creuse et inégale. Les brins les plus 
laibles seront coupés à la serpe et de bas en haul. en thute: les vieux étocs seront ravalés aussi basque possible.

Le sijour de l'eau sur les souches est la principale cause de leur dépérissement. Labatage, met tant à nu les tissus intérieurs du trone. les expose à des influences contre lesquelles ils ne sont pas prémunis. La zone de bois parfait, qui entoure le canal médullaire nayant plus qu'une faible vitalité, est d'aborel attarquée par la pourriture résultant de l'introduction des eaux qui pénètrent dans les tissus par la section. On roit alors la souche se creuser au centre. la pourriture sétendre peu à peu à toutes les couches de vieux bois, gagner les racines et finir par amener la mort de la souche, malgré la surexcitation de vitalité qu imprime aux couches externes lactire croissance des rejets.

On ne doit pas craindre de couper rez-terre, si ce n'est dans les sols bas et humides.

Les sourhes qui paraissaient à flemp de terre an moment de labatage se trouvent. apres quelifue temps. flevies de plusieurs centimitres au-dessus du sol, qui se tasse et se dénude lursigüil est exposé aux influences du soleil, de la grelée el des vents.

Les rejets 'qui viennent sur des étoes coupris trop' haut ne prennent pas de pied et nont par la suite aucune soliditi. Ceux qui poussent au nireau du sol 


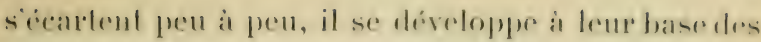
racines qui se rolient directement il loms liges : ils

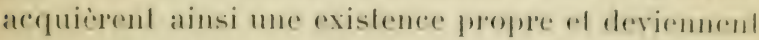

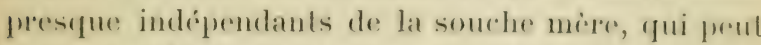
disparaitre sans entrainer leme prete. La vie des jels qui ont eru sur des éloes élevés estan confraireloujours surbordonmée à celle de la sonche. Il y a done lout avantagedadopter un mode dabatage gui assure mo durée presque indéfnie aux taillis. Malerré sos avanlages, la coupe rez-lerre ne peut ètre pratiqué partout. Dans les bois mouilleux, ou lieau couvere le pied des arbres au printemps, les souches no produiraient pas de rejets, et seraient exposées à périr si leur section se trourait au-dessous du niveau de leau. Dans les sols ainsi exposés aux inondations, il laut donc conserver des souches plus hautes que dans les terrains secs.

Les chèmes rerts ou yeuses des climats méridionaux, qui croissent ordinairement sur des collines calcaires tres arides, demandent à ètre exploités entre deux terres; il en est de mène des essences qui drageonnent facilement.

Les hètres, au contraire, paraissent donner plus aisément des rejets, lorsque lon tranche dans le jeune bois. Cette essence repousse difficilement de souchedans certaines régions de France, et lorsquion la traite en taillis, il faut avoir égard aux ha- 
bitudes locales, sous peine de compromettre la régénération. - La conservation des brins trainants qui entretiennent le mourement de la régétation parait favoriser la production des rejets; il est certain que dans certaines contrées, le Morvan par exemple, des souches de hètres àgées de plusieurs siècles conservent encore toute leur vitalité, gràce à un mode dexploitation qui consiste à ne jamais les dépouiller complètement des rejets, et à toujour's conper au-dessus du nceud de l'exploitation précédente.

Il est convenable dajouter que ce mode, plus propre à créer des haies et des bordures de champs que de véritables taillis, produit des souches énormes, sélerant à chaque exploitation et qui finissent par prendre lapparence de masses rocailleuses sur lesquelles sont implantés des rejets vigoureux quoique d'àges très diver's.

Dans quelques contrees, les exploitants, propriélaires ou marchands de bois ont laissé sontroduire lusage diabandomer aux bucherons les déchets de lexploitation : bois mort, copeaux d'abatage, souchons, etc.., qui ne peuvent entrer dans les cordes. lls ont crupar cette concession obtenir des prix de façonnage moins élevés, mais ils roient maintenant quils se sont bien trompris. Pour augmenter la quantité de ces bois d'outriers, les bùcherons coupent les brins de taillis. non rez terre, comme il est 
prescrit, mais bien plus bas, et pour rendere ce laavail plus lacile, ils se servent de loutil nommé plémonlaise, avere lequel ils délachent les brins de taillis avee une partie de la souche, ce qui compromel le recru; puis ils détachent a la sre le le sontchon dont ils exagerent la longueur. dirace a ces pratiques les bois dits dourrers, qui ne devaicnt comprendre que des produits sans valenr vénale, prement une grande importance an détriment du propriétaire dont la coupe est mal exploité et du marchand de bois qui paye en nature le bicheron plus cher que sil le payait en argent.

Il est difficile de supprimer cet abus anruel les bucherons tiement beaucoup, mais le proprictaire qui tient à ne pas laisser détériorer ses taillis droit interdire absolument l'emploi de la piémontaise at labatage du taillis. Sil peut d'ailleurs stipuler dans les traités qu il lait arec les bucherons que ces dernier's n'auront le droit de profiter daucuns déchets dexploitation et que les copeaux dabatage et les souchons enmettrés comme les autre bois seront vendus de mème, il aura mis fin à des abus qui deviennent dautant plus graves que les bùcherons syndiqués trourent dans quelques députés des délenseurs très énergiques.

Labatage se fait ordinairement après la chute des feuilles et avant la saison où la sève se met en mou- 
rement. Lépoque la plus favorable est la fin de lihiver. car les souches nont pas à redouter alors les gelées. quiles font gereer et qui détruisent l'adhérence de lécorce. Il y a un moyen très efficace et tres simple de préserrer les souches de cette cause de destruction. cest de les recourrir de feuilles mortes ef de terre aussitot apres labatage. - II faut éviter dexploiter par les fortes gelées. les butherons profiteront des moments de grands froids pour laçonner les bois abattus.

Il est important de ne pas retarder lexploitation jusifua l'époque où la sère est en mourement. Si rependant les bois sont destinés à ètre écorcés, il est indispensable dattendre le moment de la sève: cest une des conditions de ce mode dexploitation; mais alor's on devra obliger les exploitants à abattre les bois au fur et a mesure de l'écorçage. ou mieux merre a necoreer que des bois abattus. Dans certaines contrées, les hrins écorés sur pied ne sont ahaltus quia lautomme yui suit léeorgage, rest une methode des plus vicienses. Malgré lenlevement de lécore sur la tige et les grosses branches, les bourgeons des rameaux se developpent, les feuilles śalent: mais la sire quelles élaborent ne peut s. translormer en cambium, puisque lenlevement de lécorce a fait disparaitre le liber, elle se perd donc par éraporation. Les racines, ne recevant 
pas de sere descendante, ne penronl sallonger, les maliopes mutritives quelles contenatent sopuisent ol ba souche perd ainsi loule sat puissance pepureductive.

Dans les bois sommis an régime forestier, labatlage doit etre terminé le 15 arril el le facomnage des ramiers le $1^{\text {er }}$ juin suivant; ces limites ne deraient jamais ète dépassés, on les réduir ameme autant que possible dans les pays ou la végétation est précoce.

Le façonnage et le transport à trarers la coupe, lorsque les rejets apparaissent, occasioment la perte de beaucoup d'entre eux. Ces jeunes brins sont tendres et cassants; le passage des ourriers, le transport des ramiers en détruisent une grande quantité. Les retards apportés au façonnage ont des conséquences plus graves quion ne le croil généralement. La perte ne se borne pas à une année de croissance, car les rejets ainsi détruits ne sont sourent pas remplacés. Lherbe qui envahit les coupes après l'exploitation étouffe sourent les rejets qui nont pas un certain développement à la première feuille.

La vidange s'effectue pendant lété et lautomme de lannée de l'exploitation; dans les bois soumis au régime forestier, elle doit ètre terminée au 15 avril de l'amnée suirante. Les lacilités plus grandes que 
les propriétaires accordent sont très nuisibles au bon état des taillis.

Le transport des bois se fera autant que possible par les temps sees ou les fortes gelées, ce sont les moments les plus farorables pour les roituriers, ce sont aussi ceux où les chemins ont le moins à soutfrir du passage des roitures.

Dans les pays où les traineaux peuvent ètre employés, il sera très avantageux et très économique de profiter des temps de neige pour enlever les bois à laide de ce moyen de transport.

Réserves. - En définissant lopération qui porte le nom de baliurage. nous en arons signalé l'importance. C"est, en elfet, de la manière dont on prociede à la marfuedes réserves que dépendent le bon élat et la richesse d'un taillis sous futaie.

Un balivage bien fait doit tendre a conserver toujours la valeur de la futaie sans diminuer celle du taillis. Ce but est diffucile a atteindre. La physiologrie régétale nous apprend, en elfet, que tout obstacle apporté a laceses de la lumiere est une cause de ralentiscrment de la végétation. Les arbres réservés sur Jes taillis doivent donc arreter sa croissance. I)un autre còté, si lon tient à ne pas diminuer la richesse d'un peuplement, il faut toujour's réserver des balireaux, des modernes et mème des anciens en assez grand nombre pour remplacer ceux qui 
sont abalfus it chargue exploitalion. Voici les regles indiqués par la theorie ef confirmes par l'expérience, qui permellent de sillistaire à pen pris a ces deux exigences contraires.

Choisir autant que possible les baliveaux parmi les brins de semence, sans exrlure les rejels de souche qui ont le pied sain. Fe marquer que des sujuls dont lat tibe est vive ef bien renante. Plus un arbre est élancé, moins il est nuisible à ceux qüil domine. On derra néanmoins ne pas réserver des brins trop grèles, car ils se courbent ef se brisent lorsqu’ils se trourent isolés. On préfèrera les essences qui donnent un couvert léger, comme le chène, le frène, le bouleau, sans exclure cependant les hètres et autres arbres à couvert épais; mais on évitera de réserver les trembles, les bois blancs, qui donnent des semences légères, abondantes et d’une reproduction si facile que les taillis seraient bientòt envahis par les nombreux semis de ces essences de qualité inférieure. Dans les taillis de chène, on pourra réserver, au besoin, quelques pins destinés à servir de porte-graines pour propager cetie essence dans les clairières.

Les modernes derront ètre marqués parmi les baliveaux les mieux renants et les plus élancés des essences à couvert léger. On exclura de cette catégorie de réserves ceux des arbres conservẻs dans les 
exploitations précédentes qui donnent des marriues de dépérissement, ainsi que ceux qui s’étalent et écrasent le taillis.

Les anciens seront choisis parmi les modernes les plus beaux. On éritera en général de marquer comme anciens les charmes et les hètres, qui donnent un courert trop compact. A l'état de baliveaux ou de modernes, ces arbres ont pu itre réserrés sans muire a la croissance du taillis, mais lorsqüils ont acquis un grand développenent, ils arrètent sa récétation sur toute la surface quils recourrent de leur's branches.

Les réserves devront toujours ètre espacées de manière a ne donner en aucun point un courert trop épais; on les espacera d’autant plus quelles seront plus touffues. Si le sol offre des pentes prononcées, la réserve sera moins abondante que dans les terrains plats. Elle sera plus serrée si lexposition est chaude, et si le sol léger demande à ètre abrité.

La réserve doit itre plus serpéc sur les lisières que daus lintérieur du bois. Les arhres qui croissent en bordure recoivent plus de lumière et prennent plus de développement que ceux qui viennent dans le milieu des massifs. Ils protegent daalleurs le recru contre les intempéries.

Dans les sols fertiles, où le taillis a une rapide 


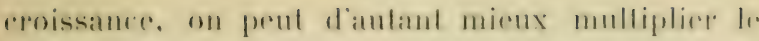
nomber des modernes a des ameiens yor ares arbres, ayant mo grande hamlemr, giveroml moins le développement du sous-bois.

Dans les lerrains maigres ol sees expusis an mili. on marquera au contraire beancomp dre haliveanx, mais lon conservera pen de modernes el emenes moins danciens, parce que ces arbres ne premant pas de hauteur, la valeme quils arefuicerem en restant sur pied ne compense pas colle quils loml perclre au taillis.

Entretien des taillis. - Les grands laillis of smltout ceux qui doirent ètre écorcés gagnent beancoup, suls sont nettoyés Dans ces nettoiements, on enlive les épines, les mort-bois et les brins lrainants; on ébranche les perches de chaque cépée de manière à favoriser leur croissance en hauteur; on extrait les bois blancs s'ils sont en trop grand nombre. Ces opérations seffectuent vers les deux tiers de la rérotion. On avance ou on retarde le nettoiement suirant la rigueur de la régétation et la raleur des produits qu'on en pour'a obtenir.

Les taillis de chène situés sur des sols maigres et sans profondeur, comme on en rencontre tant dans les montagnes granitiques, deviennent sourent très clairs. Lorsque ces bois ont été soumis à des exploitations réitérées et parcourus par les bestiaux, ils 
sappaurrissent, la bruyère les envahit et les repeuplements par voie de semis naturels n’y réussissent plus. Les vieilles souches meurent alors les unes après les autres, et comme elles ne sont pas remplacées. le bois finit par ne plus se composer que de cépées éparses au milieu de clairières conrertes de bruyères, dajones ou de genèts.

Ces taillis clairiérés occupent en France de rastes étendues; ils témoignent de lincurie de leurs possesseurs et de la négligence des per'sonnes préposées a leur surreillance. Il n'est pas farile de restaurer des bois arrivés d̀ cet état. Le premier soin it prendre, cest de supprimer la cause première de lappaurrissement du sol, le pàturage. Si cette suppression ne peut se faire en une amnée. on se bornera it mettre en défens les coupes au fur et à mesure le leur exploitation; mais on ne permettra pas aux troupeaux dy rentrer plus tard. On soccupera ensuite de regarnir les rides.

Il parait tout naturel de prendre pour cela des plants ou des graines de lessence qui forme les taillis, ef de sen servir pour reboiser les clairières. Ce procéde nest cependant ni le plus simple ni le phus economirque. Dans les sols appaurris, comme c:ux dont il sagit, les plantations d'essences feuilJues réussissent mal, et les semis sont étouffés par la bruyère. 
Le seml moyen qui ail presque comslammont ilé suivi de succes consisle it aljoinder anx rhenes des

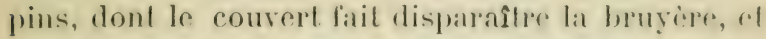
donl les aigmilles fertilisent le sol appanrej. Voirei comment on procede à celle oprepation : a lautomme qui suit la vidange de la compe it regarnir, alors que fous les bois ont élé enlerís et que le sol est encorr sillonné par les oruibes des voitures rmployées à la vidange, on ourre, dans toutes les parties clairiérées, des potets séparés l'un de l'autre par un intervalle. de $1^{\mathrm{m}}, 50$ dans un sens et de 2 mètres dans lautre. La dimension des potets sera déterminée par l'àge des plants quion aura à sa disposition. Au commencement du printemps, on plantera dans ces potets des pins de 2 à 3 ans, extraits de pépinières et soigneusement tenus à l’abri du soleil. Si l’on a eu la précaution d'établir à portée une pépinière volante d’où lon puisse extraire les plants en mottes, le succès sera prosque assuré.

En mème temps que lon plante les pins dans les trous préparés à l'avance, un ouvrier muni d'une pioche et d'une gibecière pleine de glands sèmera, au milieu de lintervalle de 2 mètres qui sépare les lignes des plantations, une ligne de glands espacés de $1^{\mathrm{m}}, 50$. On ne fait pas préparer à l'avance les trous destinés à receroir les glands, parce qu’ils se remplissent souvent deau qui fait pourrir le germe. 
Il suffit dailleurs de deux ou trois coups de pieche pour bien ameublir la terre dans laquelle on place les glands.

Il raut mieux semer au commencement du printemps qu'au commencement de l'hiver. A la vérité, on a l'embarras de conserver les glands pendant trois mois; mais comme ils germent peu de temps après le semis, ils ne courent pas autant de risques dètre mangés par les mulots et les sangliers que lorsquils passent cinq mois en terre. Au mois do juin, après les pluies du solstice, il est très utile de laire donner un binage aux plantations, pour dégager les jeunes plants des herbes qui pourraient les gèner. On répétera cette opération au commencement de l'automne, époque où l'on remplacera les plants manquants.

Liannée suivante on binera de nouveau la plantation à la fin du printemps et de lété; l'on pourra ensuite se dispenser de tout autre travail dentretien. Si lopération a été bien faite, et si d'ailleurs les accidents météoriques neen ont pas compromis le suceds, les pins doivent ifre assez vigoureux pour lutter contre la bruyère, qüils finiront bientòt par dominer. La végétation des chènes restera assez languissante tant que les pins seront petils; mais aussitòt que ceux-ci commenceront à sélerer, les chènes entreront en lutte arec eux et ne se laisse- 
roml pas dominer. A la rérolution suivanle, on

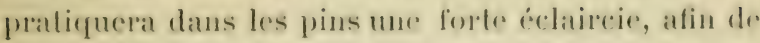
laisser les chones prender le dessus, pl, plus lard, on pourra reprendre lexploilation en laillis, aree lat

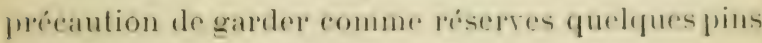
destinés à donner des semences.

Ces repenplements continués apres la viditnge de chaque coupe feront peu à peu disparaitre lous les vides. Les cépées anciennes porndeont me nomvelle: rigueme, et la bruyère sera patout chassée pal lo pin qui couvrira bientot le sol de son ombre et de ses aiguilles.

Liespèce de pin à employer comme essence accessoire pour la restauration des taillis dégradés variera suivant la région et la nature du terrain. Dans les plaines de lOuest et Sud-Ouest de la France, on pourra se servir de pin maritime; mais lorsquion avance ver's l'Est et quion s'élève dans les montagnes, cette essenee doit ètre remplacée par les pins sylrestres et d'Autriche. On préférera ce dernier pour les repeuplements à faire dans les terrains calcaires. Quant au sylvestre, il sera toujours employé avec grand succès dans les terrains siliceux et surtout dans les granits, pour lesquels il paraît aroir une prédilection marquée.

Dans tout ce qui précède, il n'a été question que de la restauration des taillis de chène dans les 
terrains paurres. Certains taillis de bois durs, situés dans les terres argileuses, fortes et de bonne qualité, présentent bien sourent aussi de rastes clairières. Cet état est sourent dù au pàturage, qui détruit les semis à mesure qüils se produisent. Dans ces conditions, il suffit le plus sourent de mettre le bois en défens pour voir les vides se repeupler naturellement. Ils se garnissent dabord de mort-bois. tels quépines noires et blanches, marceaux, riornes. cornouillers, puis, an bout de quelques années, on roit surgir des chènes, qui, après avoir longtemps langui sous le couvert épais de ces broussailles. finjissent par prendre le dessus. On facilite la croissance des chènes en étètant les mort-bois qui les entourent. Il faut se garder de les couper par le pied, car ils nen prendraient que plus de vigueur.

Quand le chène a disparu du peuplement, on ly introduit de noureau au moyen de plantations par placeaux faites après la vilange. On appelle placeau un cercle diun metre euriron de rayon quion cultive soigneusement, et dans lequel on plante trois ou quatre brins de chene. Les placeaux sont f́pars dans la coupe, mais lon a soin de ne pas les mettre sous le courert des réserres; ils sont piochés deux fois par an; les fourneaux à charbon font de très bons placeaux.

Cultures spéciales. - On désigne sous le nom 
de furelage régli un mode de traitement qui consiste: a enlever sur chaque cépée les brins les plus gros, en reservant les autres pour etre coupés aux exploitations suivantes. Ce systeme nest guipre appliqué quiux taillis de hitre, dans les montagnes du Morran et du Rouergue. Il offre cet avantage de ne pas dénuder le sol, el de maintenir lactivité de la régétation dans les souches de hètre, yui perdent sourent la faculté de produire des rejets lorsquelles sont coupées à blanc-étoc.

Pour régulariser le furetage, ou partage la rérolution en deux on trois périodes pendant chacune desquelles ies exploitations parcourent toute la forèt. Si, par exemple, la rérolution est de 30 ans, on la partagera en trois pério? es de 10 ans et on divisera la forèt en 10 coupes; dans shaque coupe on ne prendra que le tiers des brins, en chuisissant les plus àgés de manière quil reste sur chaque cépée des brins de 10 et de 20 ans; à la coupe suivante, qui revient 10 ans après, les brins qui araient 20 ans en ont 30 , ceux de 10 en ont 20 'et les jeunes rejets remplacent les brins de 1 à 10 ans. Par ce procédé, on assure la perpétuité du taillis, mais à condition toutefois de remplacer par des plantations les souches qui meurent.

Si l'on néglige ce soin, il ne tarde pas à se former des clairières, car les faînes produites par des brins 
de 30 ans sont trop rarement fertiles pour pouroir assurer un repeuplement naturel efficace.

Le sartage est un mode dexploitation à la fois forestière et agricole qui consiste à couper les taillis à blanc étoc, à brùler les bruyères et les branchages et à ensemencer en céréales le sol ainsi écobué. Ce systeme de culture nest guere usité quen Ardenne. Il permet aux populations de cette contrée dobtenir sans engrais et presque sans culture les grains dont elles se nourrissent; il active la végétation du chène qui forme le peuplement des taillis sartís, et permet den tirer dexcellentes éeorces. Le sartage se fait à feu couvert ou à feu courant.

Pour essarter à feu couvert, on pèle le solà la houe sur une épaisseur de 10 centimètres, et lion forme, avec les mottes de terre et de gazon, des fourneaux quon allume des quils sont suffisamment dessienés. Le feu dure plusieurs jours. Quand lescendres sont pefroidies. on les répand sur toute la coupe.

On pratique le sartage a feu courant en brùlant sur le parterre meme de la coupe toutes les menues branches qu'on y a laissé sécher jusquau mois de septembre. On allume ces branehages, en prenant la précaution de garantir les taillis voisins par une bande dénudée denviron 20 mètres de largeur. Pendant la duréc de la combustion, des hommes 


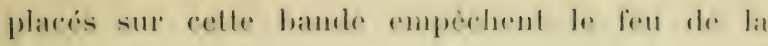
franchir.

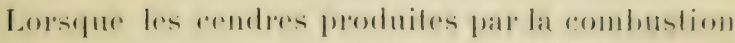
des foumeanx ou des ramilles onl ele répandues sur la coupe, on elome une cullure a la logure el lon sìme du seigle.

Le sallage a feu courert est plus muisible qure celui it feu comrant. Le feu comrant eflleure senlement les souches, et ne les empiche pas de produire lannée suivante de vigoureux rejets. Lienlèvement de toute la couche superficielle du sol, pour former les fourneaux, amène la destruction de l'humus, et, par suite, l'appaurrissement du terrain. On atténue les inconvénients de ce mode en formant les fourneaux arec les herbes, la mousse et les gazons, sans enlever des mottes de 10 centimètres dépaisseur, comme cela se fait ordinairement. Les fourneaux faits ainsi brùlent vite, produisent une cendre fertilisante, et leur confection nientraine pas la destruction de toute la couche d'humus.

Les taillis soumis au sartage s'exploitent ordinairement sans réserves. Leur régénération ne jeut done se faire que par des repeuplements artificiels.

Ecorces à tan. - On emploie au tamnage descuirs l'écorce de beaucoup d'espèces d'arbres; mais, en France, on ne se sert guère, pour cet usage, que de l'écor'ce du chène. 
Les meilleures écorces sont minces, blanches et fermes. Celles qui proviennent de taillis de 12 dे 20 ans sont les plus estimées.

Liexploitation des taillis destinés à ètre écorcés ne peut se faire quien temps de sère, cest-à-dire depuis le moment où le bourgeon s'entrourre jusqu à celui où la feuille développée commence à prendre de la consistance. Lécorcement est dautant plus facile que le mourement de la sève ascendante est plus actif; il devient impossible quand, ce mourement ayant cessé, le bois et lécorce se trourent soudés par la couche de cambium qui s'est interposée.

Dìs qüils recomnaissent que l'écoree se délache aisiment, les ourriers se hatent d'abattre le taillis et de couper les branches trop taibles pour ètre écorcées. Charue brin est placé sur un chevalet, et lon procede de suite à l'écorcement ; cette opération doit se faire dans un délai de 4 a 5 heures après labatage.

Lécorce est coupéce circulairement, à la serpe, de maniere à former des fourreaux de $1^{\text {m }}, 16$ de longueur ; puis, chacun de ces fourreaux, fendu en long, est détachí de larbre au moyen diun os taillé en forme de coin. Aussitòt que le fourreau est détaché, on le met sécher à lombre sur des branchages. Quand le séchage est complet, on forme, avec plu- 


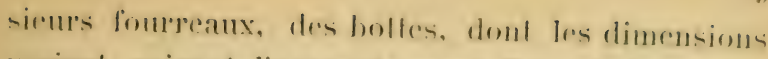
varient suivant linsage du pays. Dans le bassin de Paris, on domne aux boltes $1^{\text {m }}, 1 \frac{1}{4}$ de long sur $1^{\mathrm{m}}, 1^{\prime}$ i de rieconférence. Chacume est liée aux drux bouts el doit peser de 18 à 20 kilogrammes.

Il est tris important de ne pas laisser moniller l'écoree, car leau dissout une partie du tannin qui rt la substance à laquelle elle doit ses propriétés industrielles ; aussi certains fabricants font construire. it laide de planches ou de toiles, des hangars jour mettre leurs écorces à l'abri de la pluie.

On ne se borne pas à enlerer l'écorce des brins du laillis; on profite aussi de celle des réserves aban. données a l'exploitation; quoique de qualité inlérieure, elle troure encore son emploi.

On compte quin ourrier peut, par jour, en bonne saison, fabriquer de 6 à 9 bottes des dimensions indiquées plus hant. Quand l'écorcement se fait bien. on tire d'un stère de bois taillis jo à 60 kilogrammes d'écorce.

Les jeunes taillis de 15 ans donnent en poids moins d'écorce que ceux de 20 et 25 ans; mais sa qualité est bien préférable. Au reste, la raleur commerciale. de l'écorce varie très sensiblement suivant la provemance et surtout suivant les soins apportés à la labrication.

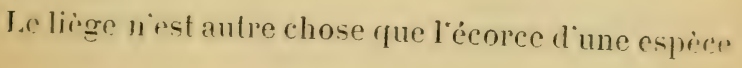


de chene que lon troure dans le midi de la Franere. ril Espagne, en Italie et en Algérie. L'écorer de ces rhenes-lieges not très épaisse, légère. élastique: mais elle n’acquiert toutes cons qualités qu'au moyen dune opération quion désigne sous le nom de démasclage. Le démasclage consiste à enlever la première? ícoree de larbre. afin de laisser se reproduire, apric cefte opération, la nouvelle icorce beaucoup plus fine qui peut seule etre employée à la fabrication des butchons. La premiere écoree, dite mâle, est employée pour fiare des bouées ef des flotteurs.

Les chènes-lieges soumis au démasclage ne sont las écorcés jusqu i laubier comme los chenes dont lience sert it faire du tan. On a grand soin de laisser intacte toute la couche du liber; car rest delle que nait le tissusuherens dont le triveloppement pureduit le liege. Onand lineree qui sest formén apues

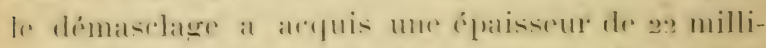
milres, ce qui arrire apres un intervalle de 8 a 1. ans, on lenlive, comme on a enlevé la premiere. Celle opération se répéle tous les 8 on 10 ans. lant que larbre conserre sa vigueur. Les chènes-lièges cariés ou mal venants sont coupés rez-trone et reproduisent des rejets quion soumet au démasclage lorsquils ont atteint les dimensions convenables.

Lat qualiti du liege dépend beancomp de létat dans

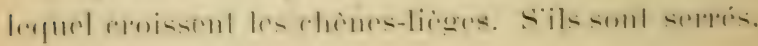




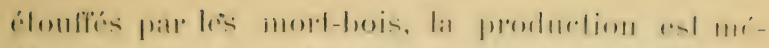
diocre a plle est abondante de de lomme qualite quamel. les pieds élanl romulieremenl espacés, los letes soml fournies of volumineuses.

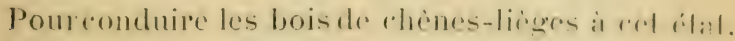
il laut en extraire les broussailles ol ne conserver sur chaque souche que les pieds les mienx venants.

Les forèts de chènes-lieges se reproduisent presrgur. toujours par les rejets de souche. Cependant, si l'on veut éviter la formation de clairières, il conviendral d'établir. dans un canton bien choisi, une petite prpiniere dans larguelle on simera des glands dre chroix.

Les plants de 3 ans au plus serviront a combler. les vides. Comme on doit craindre pour la reprise de res jeunes sujets les cffets des sérheresses prolonwés, assez ordinaires dans le pays où croit lo chineliege, il convient de fane la plantation jemelant la saison pluviense. Les trons seront profondénent difoncés et, sur la texrer quon metlra autour des ratcines, on aura soin de placer quelques pierres qui ? conserveront un peu d'humidité. 


\section{CHAPITRE VIII}

\section{FUTAIES}

Modes de trimemext. - Méthode naturelle. - Jarilinagge. - Tire et aire. - Blanc-étoc. - Gemmage. Míthode raterelle. - Coupes de régénération: sombres, claires. secondaires définitives. - Coupes d'amélioration : Yettoiements, éclaircies. - Exploitabilité. - Possibilité. - Marche des exploitations. - Repeuplements artificiels. - Abatage. - Vidange. - Jardixage. - Essences el climat. - Difficultés. Forêts de défense. - Tine Lt AIRE. - Inconvénientde la méthode. - Son abandon. - Cotpes a nu.ri:i. Toc. - Défrichement, culture et repeuplements artificiels. - Gemmage. - Quarres. - Gemmage à mori. - Produits résineux.

Modes de traitement. - On appelle fulaies les forèts dont lat régénération est exclusirement basée sur la faculte qur possident tous les arbres de - o reproduire au moyen de graines.

On traite les futaies par deux méthodes principales :

Colle du réensemenceme'nt nuturel el des écliaircies ol relle du jardinage. Liancienne méthode dite it lire el aire est complitement abandonnée maintenant, é les coupes a blanc-étoc sont trop rarement pratiquées four fulom puisice considéper eses exploitations 


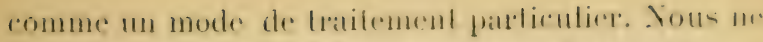
parlerons donc de ces deux demieres mélhodes que: jour dire en quoi elles consistent. Il suffira diexpuser les reggles relatives a la méthode naturelle ot an jardinage pour faire comprendre la prélérence yuion leur accorde généralement aujourlthui.

Quoique le gemmrege ne eomstilue pas nu monle. le traitement, mais hien lutilisation d’un porluil. industriel foumi par certains arberes resineux, nous avons cru devoir le eomplondere dats le chapille consacré à l'étude des fintaies.

Les arberes qu'on lirre au gemmage se reploduisant exclusirement par leurs graines, les proridés d'exploitation qui leur sont propres viennent naturellement à la suite des méthoules de culture el dexploitation dont ils sont un cas particulier.

Méthode naturelle. - La méthode du réensemencement naturel a pour objet dassurer la régénéralion par la semence et te favoriser la régétation des massifs, depuis lem naissance jusqüa lexploitation.

La premise condition pour obtenir un ensemenrement naturel est davoir des arbres dommant des graines fertiles; la seconde, qui nesí pas moins importante, cest que res graines trourent un sol apte à les faire germer et à laire prospérer les jemnes sujets qui en proviennent.

Le sol des forèts dans lesquelles lo massil a été 
romserve as nalurellement lies lavorable a lat germination des graines et à la croissance des jeunes plants. Les débris des feuilles et des brindilles accu. mulés pendant plusicurs rérolutions y forment un terreau frais el léger, dans lequel les graines et lis plauts trourent l'humidité of la tempépature, aul degré le plus favorable à leur développement. Si les arbres ne domnent pas un couveril complet. oe ferpeat se ronsomme sous l’influence de la lumiere. le sol se antre de gazon, de bruyeres ou dautres plantes qui retiennent les eraines dans le lateis fire leurs fouffes rompartes, el chouffent a leur naissince les jeunes plants qui ont pu se produire. Si. au contraire, la forel forme un massif bicn complel. si les rayons du soleil ne peurent arriver jusqu à la ferre il ny a ni gazons. ni hruyeres, le sol esl rourelt dime couche ćpaisse de lenilles mortes dans larpuelle les eraines germent of se déreloppent en foule lilerté aussile maintien du massil est-il une des eonditions les plus importantes de la regrenciration. Vous rerroms plus loin que cest encore ume des endilions indispensables it la home régretation des peuplements.

Dans leur jeunesse, les plants destines par la nalure a croitre sous le enuvert des arbres qui lem. ont domne naissance redoutent detre exposés trop promplement aux rayous du soleil, aus influemes 


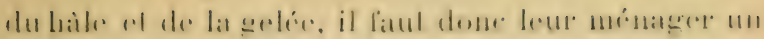
abri; daulle litrl. ees jeunes plants exigent, pour se développer, une quantité de lumière, restreinte d'abord, mais qui doit s'accroître successivement; 'ar nous sarons que lous les regelaux lirent de l'air. frur nomrilure, ef yue lem accroissement soprim serutement sous linfluence de la lumière.

Obtenir dabord mu semis complet, conserver aux jemes plants un aluri suffisant, puis enfin les li.

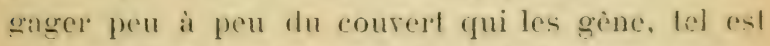

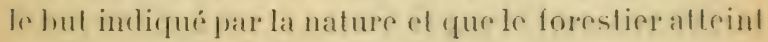
¿at moyen des compus de régénération.

Dans une premiere coupe, dite densemencemenl. on réserve un nombre d'arbres suffisant pour garnir le sal de graines.

Guand les semences sont lourdes, les rúserres dreront itre rapprochés. la compe sera sombre: wle sera claire quand les semenees sonl légerest rel sr répandent au loin, si d’ailleurs le tempéraneul lu jeune plant nexigre pas quion lui conserve un abri complet.

Les coupes densemencement dans les bois de hetles, de sapins, se font à lélat de coupes sombres. " al les jeunes plants de ces essences sont très délicats. On fera de mème les coupes densemencement dans les forèts de chènes, car le gland est lourd el ne se dissémine pas. Il importe d'ailleurs diempi- 
cher, an moment du semis, le sol de ces lorets de se gazonner, ce à quoi il est généralement disposé ¿t laison du peu de couvert que donnent les chènes. Les coupes d'ensemencement des pins peurent ètre claires, car les graines sont nombreuses, légères, et la reproduction facile.

En général, on conserve dans les coupes densemencement un courert dautant plus épais que les semences sont plus lourdes. les jeunes plants plus délicats et le climat local plus rude et plus exposé à de brusques rariations.

Lorsque le sol est suffisamment garni de jeunes plants, il derient nécessaire de les faire participer. suivant les besoins spéciaux de chaque essence, aux inlluences de la lumière et de latmosphère. Caest au moyen des coupes seconduires quion atteint ce but.

Dans res eoupes, on enlive lous les arbres qui dominent des brins bien renants, assez robustes pum se passer dabri; on éclaireit le massif de maniere a laisser. suirant le climat et les esseners. promelrer plus ou moins le soleil : on ennserve an romtraire les arbres qui surmontent les semis encore frop jeunes, les parties mal repeuplées. Lorspue les plants sont tries sensibles aux infuenees atmospluiriques (hetres, sapins), la coupe secondaire se lail ares menagement, et senlement lorsqu ils ont aequis 


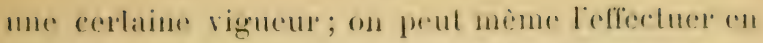
denx lois. Quand, au contraire, les jeunes planls ratgrnenl le couvert (chènes, pins), la coupe secondaire sopere aussitot que lensemencement est terminé.

Ouand enfin le jeune peuplement esl devenu assez complet el assez rigoureus pour itre deballuasio sans inconvénient du courert rui la abrité ol dont lc maintien muirait à son développement ultéricur, on le dégage, at moyen d’une coupe définilive, de Ious les arbres qui le dominent. -- On avance ou on retarde cette coupe suirant que le repeuplemenl a iléplus prompl, que les jemes sujets sont plus jobustes.

La coupe défunitive termine la série des coupes de riginération. La jeune forèt qui résulte de cettr. succession d'exploitations doit, si clles ont été bien dirigées, présenter un massif bien compact de brins serrés les uns contre les autres et à peu pris d'igale liauteul: :est là re quion nomme un fourré.

I mesure quils prennent du développement cu grosseur el en hauteur, tous ces brins tendenl it oreuper plus despace; le sol sur lequel ils sont fixés ne suffit plus à les contenir tous. Le besoin de lumière les pousse d̀ croître en hauteur; les plus vignureux surmontent les plus faibles, qui s’étiolent 1 périssenl. Leurs débris, réunis anx fenilles décom- 


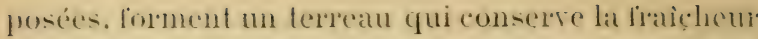
du sol et augmente sa lertilité. Le fouré passe a l'état de gaulis. Pendant cette phase de la végétation, les bois blancs, qui croissent plus rapidement que les bonnes essences et qui se reproduisent arec une grande lacilits, arriveraient a dominer lo jène peuplement si lou $n$ avait le soin d'arrèter leur pinvahissement: plus tard. on ne pourrait le faire situs inferempere le massil. Les froins domintis penrent domner déjables produits utiles: il comrixul den protiter en régularisant leséchaireies naturelles anxyuelles les peuplements sout soumis pour pasice. de l'état de gaulis à celui de perchis. et enfin de haute-futaic.

Conserver le massif en farorisant le déreloppemenl les bonnes essences ot en lirant profit des brins surabondants on inuliles, fel est le but anqual le. forestier parrient an moyen des coupes diamélio. ration.

Dans cos compes, on enlevera sucersivemenl les hrins domines. les hois hlanes inutiles an mainlien du massif, les morts-bois. Les premieres, yui soflerfuent lorsque le besoin sen lait sentir et généralement entre 10 el 20 ans, prennent le nom de nelloiements: colles qui viennent ensuite prennent l. nom déclaircies: ellessesuccident a des intervalles

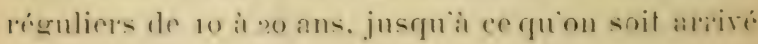




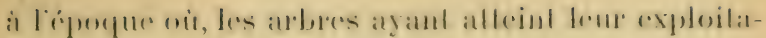
bilite, il convient de procéder ì une nouvelle rigé. nération.

Dans les nefloirments, on nenlere que les brins dépérissants, les morts-bois el ceux des bois blancs qui ne sont pas indispensables an maintien du massil. Un neftoiement trop, clair présente de grands dingers pour le peuplement. Les jeunes brins qui Ir romposenl, detant en général grèles el élancés. liont pas assez de force pour se soulenir soils ur sippuirut pas les mus sur les autres ; il lindrat dome aroir graml soin de ne pas dégarnir le massil. Les ciclaircies qui succident au nettoiement s'effectueront liaprès des rigles analogues, modifiées toutelois par la consistance plus robuste du peuplement.

Ce n'est pas tant l'espacement des tiges qui droil gnuider dans le choix des arbres à extraire que le développement de leur tìte; cest en regardant en l'ail. el non devant soi quion recommat er quil faul "xtraire ou réserver. Quand le courert est complet. les cimes bien déreloppées et se soutenant mutuellement sans sentraver, on nienlivera rien. On réserrera les arbres mème mal renants et les bois blaures dans les parties un peu claires oì le sol se courre dherbes; car cest une marque que le massif esl interrompu, el il faut y remédier. On extraira au contraire les brins étiolés et ricieux. ef mème des 
brins bien reuants lorsque le massil sera trop serré.

Des éclaircies bien dirigées conduisent un peuplement jusqüà l'exploitabilité, en favorisant l'accroissement et en améliorant la qualité des bois; elles laissent un sol bien meuble, amendé par les détrifus des feuilles, dégarni de gazon, et le plus propre enfin à une nourelle régénération.

Tel est le résumé succinct des opérations culturales auxquelles est soumise, pendant tout le cours diune rérolution. une forèt traitéc par la méthode naturelle.

Il nous reste, pour compléter cette étude, it laire romnaitre les considerations sur lesquelles lon śaprfuie pour fixer la durée de la rérolution, de maniè a ohtenir dans un temps donné la plus grande quantité des produits les plus utiles, c'est-d-dire pour détorminer lexploitabilité, et à indiquer les moyens employés pour obtenir des produits réguliers ef constants, rest-it-dire pour régler la possibilité.

Pour qu une foret se reproduise par les semis natturels. il faut que les arbese qui la eomposent srient en age de produire une suffisante quantité de graines fertiles.

Ine rette premiere comblition risulle lobligation de ne pas exploiter les parties a regénérer arant làge où les arbres commencent à dommer de bonnes graines. Comme dautre part on eherehe it produire 
des bois sains al dre bome qualite il linnl quon abatle les arbres avanl léporque oil ils dépérissenl.

Ciest entere eres deux limiles que doil itre néressalirement fixere la durée de la répolulion, corst-atdire de lintervalle qui sécoule entre la naissanee des jemes peuplements et lexploitation des bois les plus âgés.

Il ne sulfit pas de saroir qüil ne lant romper les arbres ni quand ils sont trop jeunes ni lorsquils sont trop vieux; il faut arriver à déterminer l'ìge rsact aufuel il convient de les exploiter pour en tirer le meilleur parti.

Pour cela, il laut avoir rgard au sol, an climal. a la longérité des essences, et au prix des bois suivant leurs dimensions et leurs qualités.

Dans les bons terrains, les arbres prosperent jusq11 à un àge avancé; dans les sols médiocres, leur croissance s'arrète promptement.

Aux expositions chaudes et daus les climats doux, les arbres donnent des semences plus tot que daus les climats froids. Leur fertilité s’affaiblit dés quils ont dépassé un certain âge.

Les chènes sexploitent depuis 100 jusqu à 180 ans: less sappins, épicéas, mélèzes purs ou mélangés, de go i 1.jo ans, suivant quils sont dans un sol plus ou moins favorable.

Les pins natteignent pas souvent liage de 100 
ans sans dépérir. aussi on exploite généralrment entre 60 et 80 ans, à moins qüils ne soieut lestinés à faire des arbres de mature. Les partiruliers trouveront souvent arantage à exploiter, avanl yuelles aient atteint un àge aussi avancé, certaints lutaies fournissant des menuescharpentes, desbois le $^{\prime}$ mines, des poteaux télégraphiques. ete.

Ces circonstances locales. souvent passagèites. déterminent alors la durée des rérolutions.

Régler la possibilité diune forèt, cest fixer it lavance la quantité de bois quion y exploitera, atin de nenlever exactement chaque année que le volume dont la forct saceroit penclant cette annire. Dans les taillis. on fait cette détermination trisimplement, en pariageant la forct en autant ke enmpes räil y a dianés dans la révolution of (n)

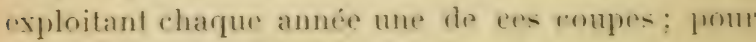
les futaies. on ne pent agir ainsi. Les conpes de rigrincration ne so suivent pas avee la regularife des roupes de taillis, cal il est sourent nécessaire de retarder les coupes secondaires ou definitires pour maintenir un abri aux jeunes plants: dautres fois. au contraire, on est obligé. de hater labatage devieux bois pour laisser croitre des fourrés que le couvert fait languir. La possibilité ne peut dom pas itre hasex sur la contenance; mais si lon aal-

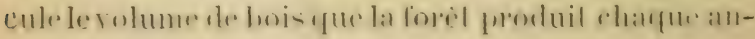




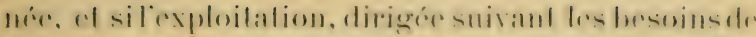

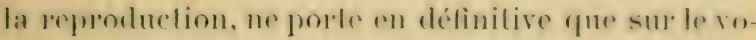
lume conmu de l'aecroissement anmure, on arrivega i

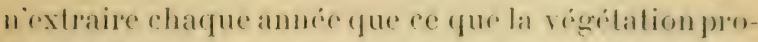
duit, el la lorè pourra imféfiniment dommer lesmemes levenus. Calleuler lo volume de bois dont saceleoil unc foret chaque annie, regler les exploitations do manière à profiter de cette quantité de produits tout en préparant la régénération, lel est le doubleprobleme à résoudre.

Pour calculer l'aceroisisement ammuel diurr loril. er qui nest autre chose que sa possibilité, on parlage dabord la forel en divisions dapres liage f.t. létat des peuplements, puis, lorsquion a bien ítabli exs divisions, on détermine lordere dans lequel il romvirendra de les régénérer, on commencant natu-

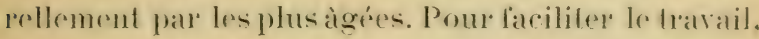
on partage la révolulion en un certain nombor de périodes de 10 on 20 ans. Lion affecte à rhacume de és périodes les divisions qui, dapres leur àge, doim rint ètre exploitées pendant sa durée, en ayant soin de rendre à peu près égale la contenance des divisions afférentes à chaque période, afin que la production soit sensiblement la mème pour toutes les périodes. Quand ce travail est fait, il ne reste quà ralculer la possibilité pour la première période com-

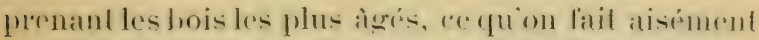


en calculant le rolume de tous les arbres de laffecfation, en y ajoutant le volume de laaccroissement probable, et en divisant par le nombre dannées de la période.

Pour faire comprendre par un exemple la manicre d'opérer. supposons quion veuille déterminer la possibilité d'une futaie régulièrement aménagée d̀ 100 ans of présentant par conséquent la série complete des ìnes de 1 a 100 ans. Il est assez difficile, on le concoit aisement, de saroir aujourd'hui ce que deviendront dans quatre-vingt-dix-neuf ans des semis d"un an, dans quatre-ringt-dix-huit, ceux qui ont deux ans, ete. Mais si lon admet. ee qui est plausible, que ces semis, renant dans des conditions semblables à celles sous linfluence desquelles ont crù les arbres àgés de 100 ans aujourdhui, donneront une production sensiblement égale à la leur. il suffirade comnaitre la quantité de bois produite par ces arberes de 100 ans. Ce serait facile si le bois arait ile rxploite par coupes sucessires comme un taillis. ef repeuplé artifieiellement lannée qui suit l'exploilation: mais nous arons ru que la régénération naturelle ne soperre pas ausisi régulierement. On ne sait pas au juste dans quelle année on abattra tel ou tel arbre, parce quion ne sait pas quand lensemencement sera produit et quand les jeunes plants pourront se passer diabri. Il faut done employer un 
antere moyen que relui on usige pour delerminep la possibilite des faillis ou des futaies par coupes it blanc-citoc. Voici comment on procide :

On admet que dans une période de 20 ans, fan cxemple, le réensemencement de fous les cantons quiont aujourdhui de $s_{1}$ a 100 ans sera terminć. et que dans cette période on aura pu abattre tous les arbres compris entre ces deux àges. On cube done fous ecs arbres, on ajoute au rolume obtenu lia:croissement probable, et lion divise le tout par vinst. Le résultat de la division donne le volume annuel que produiront les arbres de 81 a 100 ans pendant les 20 premières années de la révolution. Comme les bois qui ont aujourdhui 61 a 80 ans auront i lexpiration de cette première période $8 \mathrm{r}$ à 100 ans et qu'on les suppose dans les mèmes conditions. on admet quils fourniront un volume égal à celui déterminé pour la premiere affectation. Au reste, pour iviter des différences trop grandes, on recommence a calculer la possibilité pour chaque affectation.

Les opérations que nécessite la détermination de la possibilité servent en mème temps à régler la marche des exploitations. On roit en effet que si pendant la première période supposée de 20 ans on enlève chaque année, dans les divisions formant la première affectation, le vingtième du volume des arbres qui s'y trourent, à la fin de cette période il 


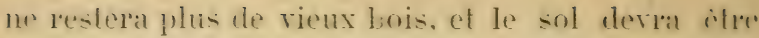
garni de jeunes plants. On passera alors aux dirisions affectées à la deuxieme période et on les pxphitera de mime. de telle sorte yuiaris lexpiralion de cetle nourelle periocle toms les vieux bois de laffectation correspondante seront remplacis par des jeunes. Si lon continue ainsia ahattre suecessivement les rienx bois de chacune desaffectations. il arrireria un moment où tous les arbres qui st trouraient dans la forèt. lorsqu on a attarpué la prenière aftec. tation, auront eté exploitis ct seront remplacés par une nouvolle génération. Ce moment coïneide avec la fin de la rérolution.

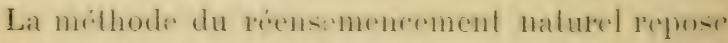
-ill liypothese que charpur affectation sera chlie-

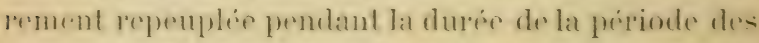

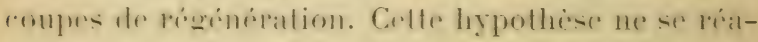

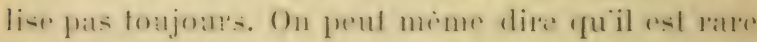

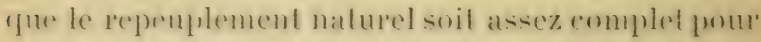
sutrire a conslituer un masif regulier. Il taut alors supplier par dos repeuplements arlifiels a ce que lensemencenent nalurel na pu faire. On ne loit pais allendre, pour commeneer ces repeuplements artificicls, que toute laffectation dans laquelle ils

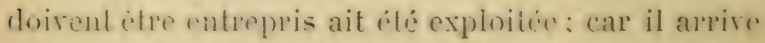
4mulenl y

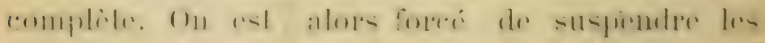




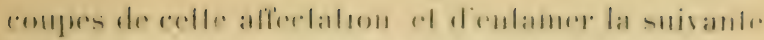

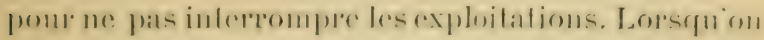

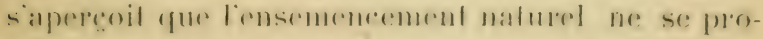
duil pas, il faul se mellre en mesure de garnir la roupe soit au moyem de semis, soit par des plantattions.

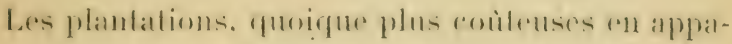
rence que les semis, revienent sourent moins cher. paree quelles ont moins de chances d'insueces. Ponpro quon puisse se procurep de bons plants, om

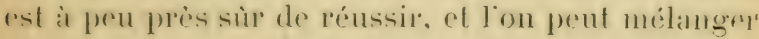
les essences dans les proportions les plus ronvemables. Ce mélange d'arbres aptes à virre cusemble est un des moyens les plus certains dassurep la bonne renuc des futaies.

Ainsi, on plaine dans les lemains legers. le rhene associé an pin sylrestre rooil mirux qua labl fur. Dans les sols plus comparels, lo rhene sassorcie au charme, a lorme, aux érables. En monlagne, les massil's mélangés de hetres et de sapins ont une plus belle renue que ceux où ces arbres croissent sans mélange. Les repeuplements artificiels permettent dintroduire dans les forèts les essences qu il est utile dy propager; ils serrent à empècher la inrmation de clairières et complètent ainsi l’ouve le Ja nature, dont laction na pas foujours la rénularili que comporte mu aménagenent hirn suivi. 
En mème temps que la première affectation se repeuple. soit naturellement, soit artificiellement. les affectations suivantes sont parcourues par des coupes d'amélioration, éclaircies et nettoiements. Cus coupes régularisent les peuplements, préparent la régénération et permettent de tirer parti de tous les bois surabondants. Comme elles peurent se' suivre dans un ordre bien déterminé, on les assoit par contenances égales sans se préoceuper des différences des produits. Il nous resterait maintenant atracer la marehe des coupes tant de régénération que damélioration: mais nous ne pourrions, sans dépasser les limites de ce travail. entrer dans lexamen de ces questions qui sont du ressort de la science de l'aménagement plutòt que de la sylviculture proprement dite.

Labatage, dans les fulaies, doit seffecturer apris la chute des feuilles el avant l'époque ou la sève se met en mouvement. - On eroit en général qui les brisexploités en frmpls de seve, ainsi que ceux uqui sonl coupés hor's des époques de la pleine lune. ne se conserrent pas. Aucune expérience concluante ne légitime cette croyance rulgaire. Si l'on prescrit de faire les exploitations en automne et en hiver, éest principalement pour attépuer les dégaits causés aux jeunes peuplements par des abalages faits an prinlemps of en éle. éporgur oules 


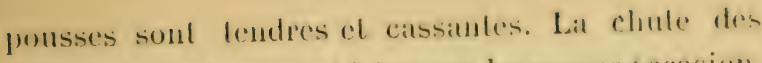
arbres ef leur transport à travers les recrusocoasionnent alors bien plus de dommage que dans larrière-saison.

Dans toutes les coupes de régénération, on ébranchera les arbres à abattre et lion dirigera leur chute: de manière à éviter d’endommager le sous-bois.

Dans les coupes de cettle nature, on n'a pas à mémager les souches comme dans les laillis. On pent douc permettre l'usage de la seie pour l'abatage des gros bois. La scie quiest la plus commode pour ce travail est celle dite Tyrolienne. C'est un passepartout dont les dents sont découpées sur une lame convexe. Quand on veut profiter de toule la longueur de certaines pièces de prix, on procède à l'arrachagro 'u creusant autour du pied un fossé circulaire. On tranche les grosses racines à mesure quion les dicouvre, et quand l'arbre est à peu près détaché, on le met à bas au moyen de cordes. Cette opération doit toujours ètre précédéc d’un ébranchement complet.

On façonnera les branchages le plus tôt possible et on transportera les bois sur les laies, chemins et places de dépôt. On profitera des fortes gelées ou des sécheresses pour faire ces transports, beaucoup plus faciles alors que le sol est solide. On sabstiendra de faire pénétrer les voitures dans les coupes après les grandes pluies et les dégels. - II 


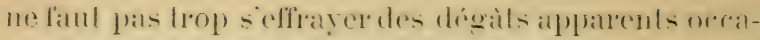
sionnés par les exploitations : les jeunes peuplements de bois feuillus qui paraissent dérastés après la coupe se complètent assez promptement. si on a soin de leceper les brins endommagés. Les résineux demanlent à ètre mieux ménagés; cependant. il nì aul:a fas de danger sereieux pour leur avenir. si lou a la frécaution de ne pas faire dir sermules elairieres dalls les jeunes fourrés.

L abablissement de honnes roirs par lespuelles lis probluits de toutes les compes deviennent aisement laansportables est sans rontredit laméliomation la plus profitable aux forcts: malheureusement, ces lravaux exigent des arances derant lesquelles hean-

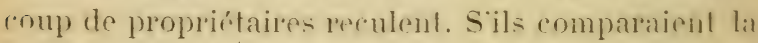

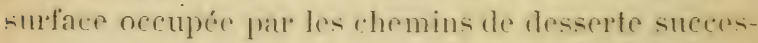
sirement ouverts puis abandommes a celle quiocemperail un ben réseall de chemins, sils tenaient comple de la diminution des frais de transport ef par suite de laugmentation de raleur des bois, ils pecommailraient presque toujours que les linis de promier

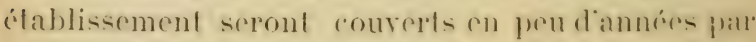
la plus-ralue des coupes.

Les produits des coupes situésen pays accidentis. ou les roitures ne peurent arrirer. sont transporlí soit au moven de glissoirs. soit sur des chemins de scinlille. 


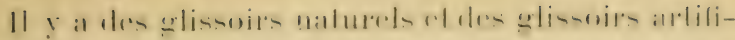

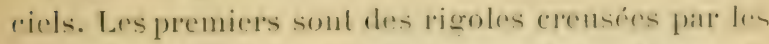
ranx sur des pentes raides el quion redresse le. mieuxpossible; les autres sont construits at moyen

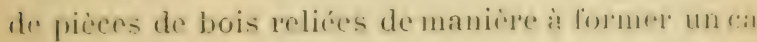

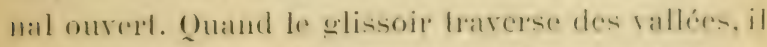
est supportí par des éfriers ou des relevaleds. Lixtremite inféreure des gliscoirs débouche ordi-

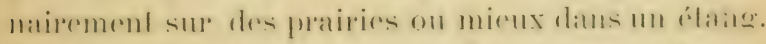

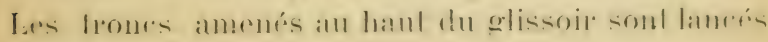
le gros bout en avant.

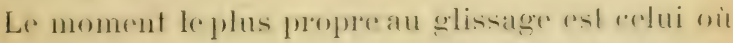
la neige est wien prise; mais on doit suspendre le lancement pendant les grand lioids. parce que les frones se brisent alor's aved unc grande facilite.

On appelle chemins de schlille des sentiers rn la:vers destuels se placent des morecanx de bois retenus par des picpuets. Dans les Toseres, on nomme rupetons. Jes bùches qui forment les barreaux de lispremere d'íchelle yuon élablit ainsi sur toute la longurum. du chemin. La pente du chemin de sehlitte est de 10 a 12 pour 100, elle doit ète aussi régulière que possible. Pour transporter les bois sur ces rhemins, on se sert d'un traineau léger tiré par un homme qui appuie ses pieds sur. les ravetons.

Jardinage. - On appelle jarlinage un systeme dexploitation des futaies rui consiste a enlerer a 
.1 Lit dans loute la lorét les arbres les plus àgés. les plus viciés, secs ou dépérissants, et mème ceuxbien renants que réclament les besoins du commerce.

Ce mode de traitement est celui qui a été le plus anciennement pratiqué, car lorsque les hommes ont commencé à employer le bois pour construire leur's premieres demeures, ils ont abattu dans les forits les arbres à leur convenance, sans se préoccuper dr lem reproduction. Comme les forets étaient immenses et la population clairsemée. ces exploitafions faites sans règle navaient pas de grands inconvénients, car la puissance de reproduction était supripieure a la consommation. Mais il rint un moment où les besoins sacerurent au point de comfromettre lexistence des forets, qui ne produisaient plus assez de bois pour satisfaire aux exigences des populations. Dans la crainte de roir disparaitre les forets et arec elles le bien-itre quelles produisent. les gouvernemonts prirent des mesures pour assurer lenr conservation. Le jardinage désordonné fut infortit dans les forets possedées par les sourerains et Jes rommunautes at lon fixa pour chaque foret le nombre diarbres a abattre rhaque année. Les particuliers adoptirent cette rigle. qui leur assurait un revenu soutenu, ef aujourdhui on ne jardine plus it laventure que dans quelques régions peu accessibles où les bois n’ont aucun débouché. 
Le jandinage regulier consiste done is exploilere ehaque année, ou da des périodes fixes, 10 nombre déterminé darbres choisis dabord parmi les sujels morts, viciés ou dépérissants, nombre qui est complété arec les plus gros des arbres sains.

On concoit que si le nombre d'arbres ainsi exploilis est fixé de manière à ne pas dépasser laccroistement ammel de la torel, relle-ei ne sappantrima pas. Si d'ailleurs ces extractions sont disséminees sur de grandes surfaces el n'enlérent qu'un ou deux arbres sur charque point, le massif ne sera interrompu nulle part et les jeunes plants seront toujouis abrites.

Cette dissémination des exploitations, qui est une des conditions nécessaires du jardinage, n est pas sans quelques inconvénients. La surveillance est. plus difficile, les transports sont plus onéreux pour des extractions dispersées dans toute une forèt que pour des coupes faites din seul bloc, comme celles des taillis. Aussi a-t-on cherché à atténuer ces inconrénients, sans toutefois modifier le caractère essentiel du jardinage, en concentrant les extractions d’arbres sur des surfaces réduites. Pour cela, on partage la forèt en un petit nombre de divisions de contenance à peu près égale. Ces divisions sont établies en utilisant les limites naturelles, vallées, routes, crêtes. Leur nombre varie suivant la disposition des lieux et la fertilité du sol. Les exploita- 


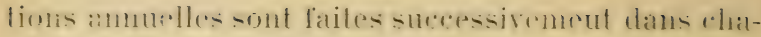
cunc de ces dirisions, dans une périote égale à leur nombre. Sil y a 10 divisions. les exploitations reriendront tous Ies 10 ans dans chacune d'elles. Ces (ryploitations portent d'aliord sur les arbes morts.

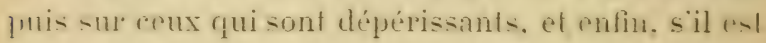
maresalire pour pardaire la possibilite. sur les arberes les plus murs. Mais elles so font toujours par piph

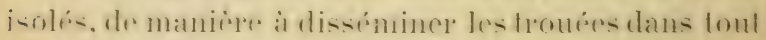

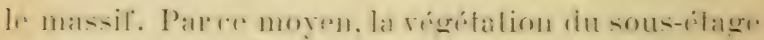

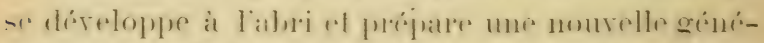
ration de grands arberes. On arrire ansi it obtenire sur charfure point de la foriet des arberes de tout alse yni se souliemment les mos les anters el qui forment fartoul un pertplement miformement rarif.

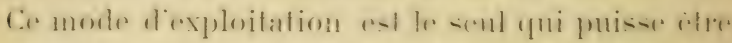

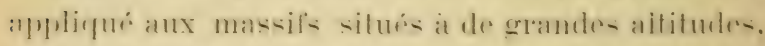

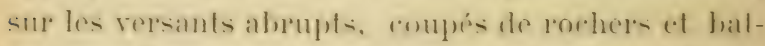
lims par les rents. ainsi qua a cens qui constitment

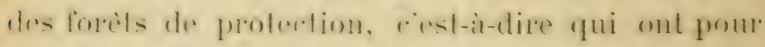

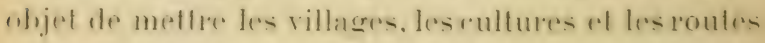
a labri des avalanehese des aboulements et des rents dangerenx. Il est eneope indigur pour les lurets dans lespruelles, à raicon de la rigneur du climat of la natupe du sol. la rigendration naturelle est extrèmement lente ef aléatoire.

Enfin le jarrlinage est lo senl mode applicalule. 


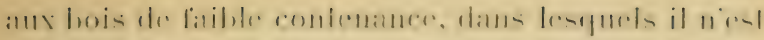

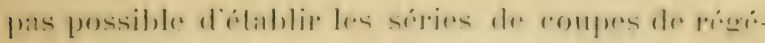
míalion el diamélioralion que comporte la melhode du réensemencement el des iclaircies.

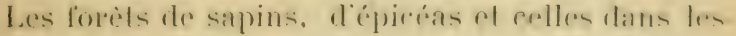

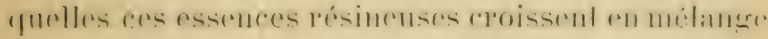

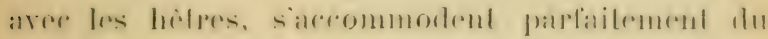

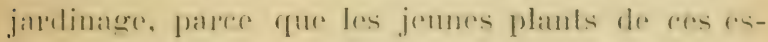

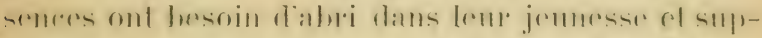

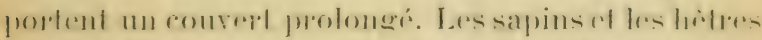

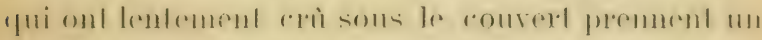

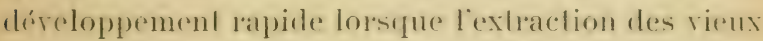
arlores qui lis dominaient leme domme nu pend de jour?

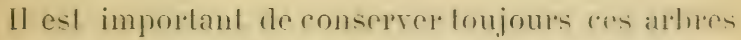
dominés qui constituenl le soms-bois dans lerfuel slo rermule la fulaie. On derata se bormer, bous de lies-

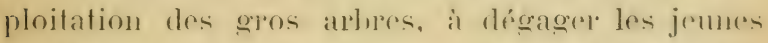
sujelstropétonflís par les essencesserondaires, mais il latuf se garder de détruibe systématiquement res essences, dont la présenerest trix utile pour mainfenir la liaichen du sol ol lavoriser la germination des graines et la rroissanre des jemes plants.

Les forèts de mélèzes el de pins cembros ne pourraient impunément supporter les roupes de régéuŕration faites suirant la méthode naturelle; car aux altiludes où eroissent les massils de ces rssenres. il 
est indispensable de couserver toujours sur les pentes des arbres assez vigoureux pour retenir les roches roulantes et les neiges.

Le jardinage est donc le seul mode de traitement applicable à ces forèts, qui d'ailleurs s'en accommodent fort bien; car les arbres dont elles sont tormées se reproduisent mieux par touffes isolées que par massils uniformes.

Le rẻglement de la possibilité des forèts jardinées ne peut pas se faire par les procédés employés pour des forèts traitées par la méthode naturelle; mais. lobservation des massifs fait apprécier assez exactement leur état, pour quon puicse roir si les abatages sont en rapport avec laccroissement. Apres quelques tàtonnements, on arrive à déterminer ì peu près exactement le nombre de vieux arores quion peut abattre par an. sans que la forèt en soit appaurrie.

Ces abatis de rieux arbres fails ea cot lid, en jardinant, dans les parties où il existe déjà de jemnes hois, doivent ètre suivis de nettoiements destinés à enlerer les brins hrisés par lexploitation el les sujets mal venants, nuisibles an jeune penplement.

On peut fixer assez exactement la possibilite des forits jardinées dans lesquelles les exploitations se succedent sur des divisions peu nombreuses assises 


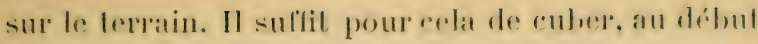
drehaque pérode, lous les ardores de fulaie ayant

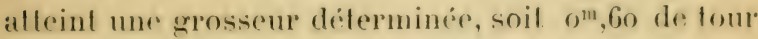
i hauteur dhomme. Les résultats de ere cubagre sont inscrits sur m carmet, par catégorie de grosseur et pour chacune des divisions. Pendant la premiere rolation, on extrait, tant en arbres morts qu sujets sains, la quantite de metres cubes quion suppose devoir etre produite par l'aceroissement du matériel cubé, soit, par exemple, ́ pour 100 du cube total par an. On recommence à lexpiration de celte période le cubage de tous les arbres de $\mathrm{o}^{\mathrm{m}}, 60$ de tour et l'on compare le chiffe obtenu arec le résullat du premier cubage, en tenant compte du volume des arbres exploités, des chablis et des arbres morts. Cette comparaison permet de recounaître si le taux dé pour 100 empiriquement adopté est trop fort ou trop faible.

Si lon voit que lon a un peu trop compé pendant la période, on abaisse un peu le taux pour la période suivante. On l'élève, au contraire, si l’on reconnaît que le volume exploité est inférieur à l’accroissement. Ce contròle permanent des modifications du matériel donne un moyen sùr de régler l'exploitation d'après l'état de la forèt; il permet en outre, quand les carnets sont tenus arec ordre, de connaitre lacoroissement relatif des arbues des 
diverses rategories of de faire porter les abatagrs sur ceux dont la croissance se ralentit.

Cette méthode. qui porte le nom diaménayement controles ou de méthode frunçaise, est encore peu ronnur. mais elle mérite dedre adoplér par les parliculiers propróétanes de fulairs. pare quelle leur furmet de se rendre un compte exact des lluctuations qur les coupes produisent dans la richesse des peuplements.

Tire et aire. - Le mode d'ixploitation dit a tire el aire consistait a diviser toute la foret en coupes degale contenance, quion abattait successiremenl. roleserant un eertain nombre de porte-graines. Cette methode ne diffirail do celle du taillis que pal lat durée des refolutions. Dans las forrets traitées a

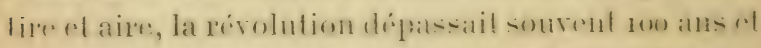

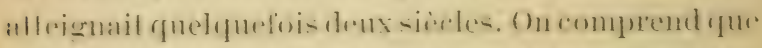

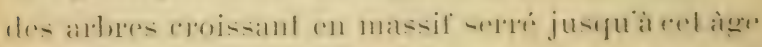

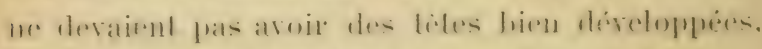
romme il convient at de bons forle-glatines. et fuw lenrs sonches ne deraient pas fournir beaucoup de rejots apres lexploitation. Aussi lat rénération étaitalle tres sourent insulfisante. Les bois blanes envahissaient sourent les forits ansi traitees, el quand 10 sol netait pas de bome qualite, il sy repit de. rastes clairieres.

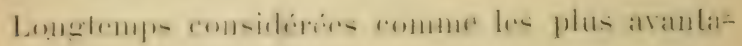




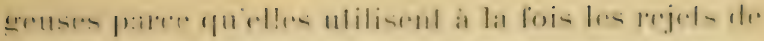

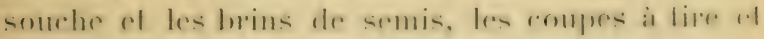

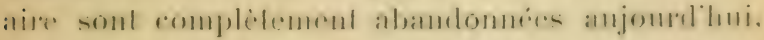
parre y

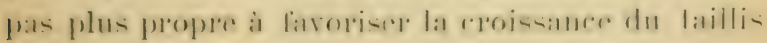
que celle des liulaies.

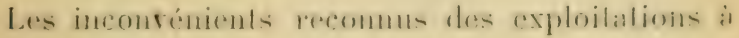

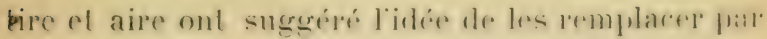

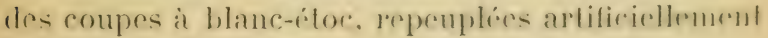
après culture du sol.

Blanc-étoc. - Ce molve dr Irailement nesl nalí

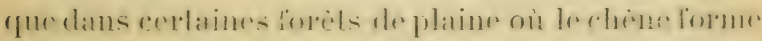
lissence dominante. Les repeuplemente allifirels. 'xécutés mélhodiquement apres une pécolte ou dems dre cépéales, ont une rigularité qüil res impossihl. dallender de lemsemenermont nalurel. Labalacer

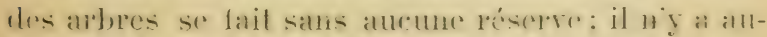

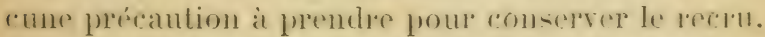

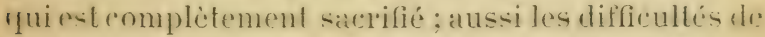
lexploitation sont-elles beaucouly moins grandrs rue dans les coupes traitées par la méthode naturelle.

Iais ce procéde de culture des forets nest applicable que dans les terrains peu accidentés, où le sol est assez profond pour niaroir pas à redouter les suifes diun defrichement romplet. La ralete dre

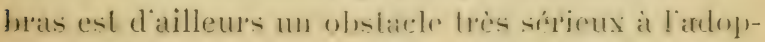


tion d'une méthode qui exige une main-d'ceurre très considérable pour le défrichement et la plantation artificielle de toute la surface des coupes annuellement exploitées.

La méthode des coupes ì blanc-étoc, suivies de repeuplements artificiels et déclaircies, a un grand mérite, celui de la simplicité; il doit suffire pour la faire adopter, quand dailleurs elle nentraine pas a des dépenses exagérées.

Gemmage. - On appelle gemmage lextraction des produits résineux que fournit le pin maritime. L'industrie du gemmage a pris depuis quelques années une grande importance; rest la source de la richesse de plusieurs départements du littoral de l’Océan.

Voici comment on procede à cette opération. qui commence quand les pins ont $2 \bar{j}$ ans, àge aupuel ils deviemnent bons à gemmer. L'n ouvrier muni d'une law:hette légirement courbe pratique. rers le pied de chaque arbre, une entaille de 12 a 15 centimetres de largeur, sur 35 a jo centimetres de longueur. Cetle cntaille nommée quarre va jusqu'à laubier; la résine qui s’éeoule par cette plaie est recueillie soit dans de petits godets, soit dans de petites caritis creusces au pied de l'arbre. Chaque semaine le résinier rafraichit la quarre, qui s'allonge successive-

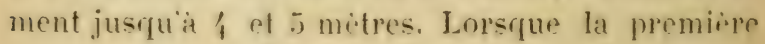


fuare est arrive a colfe hauteur, on en fait une seconde sur lautre face et on continue ainsi jusqu a ce que les quarres fassent le tour du trone. Vers l’àge de 60 ans, les pins sont gemmés à morl, c'esta-dire quion ravive toutes les quarres, et quion en ourre de nouvelles partout ou il est possible de le: laire. L’arłre ainsi épuisé par l'écoulement dr la résine est ensuite abattu et débité en planches, rn ichalas, etc. La résine distillée fournit l'essence de lírébenthine, les brais, le galipot et tous les produits dérivés de ces substances. 


\section{CHAPITRE IX}

\section{REPEUPLEMENTS}

Modes de nePetplemeyt. - Semis, planlaitions. buntures et marcottes. - Comparaison des dirers morles. - Semis. - Choix des graines. - Récolte et conservation. - Préparation du sol. - Semis en plein, luar bandes et par potets. - Semis sur la nẹige. - Binages. - Plantations. - Saison favoralıle. - Extraclion des plants. - Précautions contre le soleil et le froid. - Mise en jauge. - Pose du plant. - Recepage. - Périxières. - Choix de lemplacement. -- Première préparation - Clóture. - Division en platesbandes et rigoles. - Abris. - Entretien. - Pépinieres volantes. - Bottrne- - Essences propres an lunturage. - Modes dexerution. - Mareottage.

Modes de repeuplement. - Un repeuple les larrains degarnis de bois an moyen de semis. de plantations, de bontureset de marcottes.

La nature du sol. lespece d'arbres quion reul ! introduire déterminent le choix à faire entre ces divers modes de repeuplement.

On emploie les semis lorsquil s agit de reboiser des terrains pierreux où les plantations sont dime exécution difficile, les terres légères garnies de

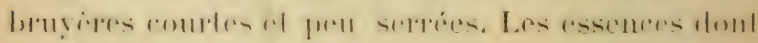




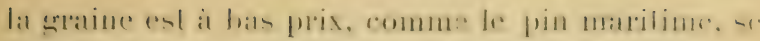
siment plutiot quielles ue se planuteul.

La plantation est preferable anx semis, dans les terrains compacts, courerls dherbes ef exposic an soleil; elle est senle praticable pour le repeuplement des vides de peu distendue. En gémépal, me plantation bien late offre plus de rhances de reussite qu un semis, el ne coùte, en fin de emmple.

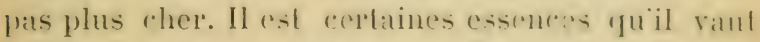
mieux semer que planter; ce sont celles dont les blants sont fortement enracinés, comne le chene, l. rhàtaignier. Les diffeultés quon éproure à extrair: les plants de ces essences et a les mettre en terre arec leurs longues racines rendent les plantations si coùteuses que le semis. malgrés ses inconvinients. doit elre preferé lorsqu il sagit de lravaux de qualque importance.

Les repeuplements jar boutures somb limités an frès petit nombre diessences qui se reproduisent aisément par ce procédé. Le marcottage peut sappli'quer à un plus grand nombre despeces darbres, mais il exige de tels soins quil est difficile de l'enployer pour des reboisements importants. Ca mode; tris utile pour propager des arbres précieux, est du domaine de lhorticulteur plutìt que du forestier.

Semis. - Les semis se lont au printemps ou en

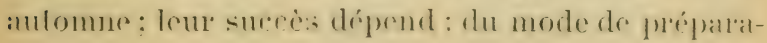


tion du sol. du choix des graines, de leur mise en terre, des soins apportés ḋ la conserration des jeunes plants. et enfin des circonstances climatériques, qui nf peurent ètre toujours combattues, mais contre lesquelles on peut eependant prendre quelques precautions.

Les graines doirent etre saines, fraìches et de bonne qualité. On s’assure de leur qualité en les ourrant à laide de longle ou du couteau: elles doirent ètre pleines a lintirieur et présenter les caractires dine graine récenment cueillic. Ces caracteres rarient suivant les essences; nous les ferons connaitre spicialement pour les semences qui sont d'un emploi général, en indiquant les soins à donner à leur récolte et à leur conservation.

Le gland doit itre plein, lourd, lrais a lonterient el mumi de son germe; les grlamets piqués, moisis.

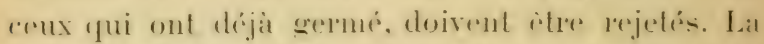
recolle se lait ch atitomne. par un temps sec sil est possible; on évite de choisir les glands qui proviemnent darbres dominés ou trop àgés, ou ceux qui sont tombés les premiers. Aussitòt après la cueillette, on les laisse se ressuyer dans un lieu sec et bien aéré.

Quand on sème les glands en automne, il 11'y a dautre soin a prendre que de les étendre et de les remuer a la pelle pour yoils ne fermentent pas : si 
lon reul les comserver jusquian prinlemps, il linul les mélanger arec du sable bien lavé et sec, les mellw en tas sur une aire bien ballue et les recouvrir diuns couche épaisse de paille ou de fenilles siches; il est préférable de les garder en silos ou daus leau. On ronstruit les silos en onrrant, dans un sol bien a labri des eaux, une fosse assez prolonde dont on garnil le fomd diun lit de paille de 30 centimitres d'ípaisseur: les parois sont reretues de pienx entrelatés de tresses de la mème matière. Les glands sont ilendus dans les silos par couches que séparent des lits de paille ou de feuilles sèches, et lon recourre le tout de planches ou de branchages sur lesquels on rejette la terre.

On peut aussi conserrer les glands en les meltant rans des tonneaux quion remplit d'eau. Dans certains pays, les gardes emploient ce procédé jour obtenir à peu de frais une boisson rafraîchissante el assez tonique. La légère fermentation qui šétablit ne détruit pas la faculté germinative du gland; quelques poignées de houblon jetées dans le tonneau donnent au liquide, quion retire au printemps, un soùt d'amertume qui n’est pas désagréable.

La faîne, qui est le fruit du hètre, doit ètre pleine, fraîche et d'une sareur franche. L'on en tire une huile de très bon goùt. On récolte la faîne en automne; il faut attendre qu elle tombe naturellement. 


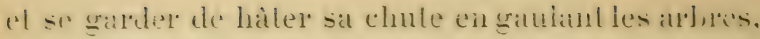
1.nir on détruit ainsi les bourgeons qui fructifierairnt "anmie suirante. Apris avoir laisse se ressuyer les gritines recueillies. il sulfit de les entasserilons uir chambre aérée et bien siche. et de les recourrir d'une couche de paille.

Les graines des épicéas el des pins sylrestres se riroltent en automne ef en hirer : celles du sapin ('n septembre. On cucille les connes a la main, on les entasse dans un erenior aépé et on les remue de tempe en temps. Les graines du sapin tombent aver. les écalles: on les sipare par un epiblage. Le; arailles des cones des ipicias el des pins syluestres ure somrent yar sous linthene de lat chatean. Lextration en crand sopere andiliedellement dints des appareils sperians. Pour les quantités pen ront1siderables, il sullit dexposele les eomes all sol:ail of de les remuer: apres queldues hemes. la ermatre séchappe avec facilité.

Les semences anusi pecueillies sonl garnies dimu membrane légere qui on far:-ilite la dispepsion : il $:-1$

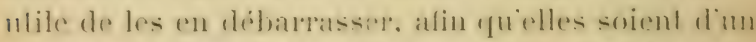
moindere volume et que le vent ne les enleve pais ansid aisimant. (On exiente cette opération en lis halfant au hiau apries les avoir renfermees dans un sace ou meine ea les agitant fortement dans un batrillet oi lon place puelipues eailloux. 


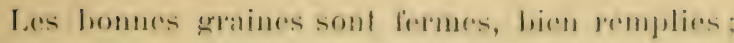
leur sareur est franche ef résincuse. La graine de lípicéa est un peu plus grosse el plus allongée que celle du pin sylvestre, elle est d'une couleur moins foncée. Comme elle est beancop moins chère, les marchands la mélangent sourent avee cette dernicre, aptris lavoir coloris en brun foncr. Pour rexomnaîle ecefle fraude, il suffira de prendere quelfurs graines ef de les semer dans un vase rempli d'un lerreau léger quion arrosera souvenl avec do l'eau tiède. La germination sopérera promptement it permettra de distinguer les pins sylvestres, qui naissent avec 5 ou 6 feuilles, des épicéas, qui onl presque toujours g feuilles séminales.

Les cônes du mélèze sont mûrs à la fin de laulomme, mais la dissémination ne se fait qu'au printemps suivant. Beaucoup de ces graines sont stiriles, On récolte les cònes pendant l'hiver.

La graine diorme est mùre au commencement de juin; elle se dissémine aussitôt. Cétle graine esl tris abondante, mime chez les jeunes sujets: mais elle est trìs souvent vaine.

On récolte après la chute des fenilles les graines du charme, des érables et les chatons d'aune.

La graine de boulcau est mùre en aoùt et septembre. Comme elle est très petite et pourvue d'ailes, sa dissémination est rapide. Les semences do. 
lonleau sont tres sourent raines, aussi est-il lorl rare de voir réussir les semis artificiels de cette essence; mais comme elle se propage très bien naturellement et que les jeunes plants ne sont pas très fortement enracinés, on peut aisément trourer dans les bois des sujets propres à la plantation.

On prépare les terrains suirant que le semis doit stre lait en plein. par bandes ou par potets. Dans lo premier cas, le sol est entièrement cultivé à la rharrue ou à la houp. On emploie la charrue dans les terrains en pente douce ou en plaine, lorsque le sol n'est pas embarrassé de roches ou de racines; on cultive à la houe les terrains accidentés ef ceux en général dans lesquels on ne peut sc sorvir de la charrue. Il est parfois arantageus. pour débarrasser romplitement des mauraises herbes le terrain a reboiser, de faire, arant le semis des essences forestières, une récolte ou deux dr. réréales quion fait suivre d'une récolte de plantus sarclées, telles que pommes de terre, navets, etc.: on sime ensuite les graines forestières, après aroir. domné une légère lacon at la terre. Mais ce sont des moyens qui ne penvent ètre employés que dans les sols de honme qualité et dans les pays où la populalion agricole est nombreuse, conditions qui se prisentent rarement dans les régions boisées. Le semis en plein est une ngripation couteuse. surtout lors- 
'puil sagil de défricher des terrains argileux forlement gazonnés. Dans de pareils cas, il est plus économique de semer par bandes ou par potets. Mais on peut, sans dépense exagérée, semer en plein sur un terrain léger, couvert de feuilles mortes, de bruyires courles el clairsemés ou précédemment cultivé, parce qu'alor's on se borne à donner une légère façon au moyen d'un trident en fer qui écorche le sol et en ameublit la surface.

Le râteau ordinaire à dents de ler n'est pas assez solide pour cet usage. Ses dents trop courtes égratignent à peine le sol; elles s'ébranlent d'ailleurs au bout de quelques instants et finissent par n'avoir plus d'action. Le trident recourbé (fig. 45), bien plus maniable et plus solide que le ràteau, attaque assez énergiquement les terrains les plus durs, et les sil-

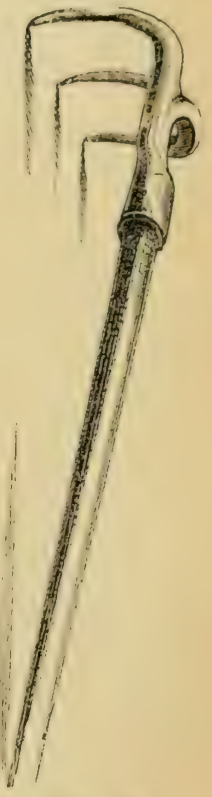

Fig. 年. lons quil trace sont assez profonds pour loger les graines et les abriter.

On prépare le terrain pour les semis par bandes alternes, en ouvrant, soit à la charrue, soit à la 
houe, des lignes paralleles espacées de tou centimitres à 1 métre, ef d'une largeur de 30 à jo centimitres. Les gazons qu'on extrait des parties ainsi cultivées sont rejetés sur le bord de la partie restér inculte, de manière à former un bourrelet qui garantit les jeunes plants de l'ardeur du soleil et consire la fraicheur à leurs racines. Dans les pentes. .. bourrelet doit itpe établi sur le bord inférieur dr la bande cultirée, celle-ri doit etre parallele it l'horizon.

La préparration pour les semis par potets se lail it la houe; on enlève les gazons de place en place sur une surface de jo à 60 centimetres de còté, on les rejette sur le bord du potet ef l'on dome une bomr facon an terain ainsi dénudé. Ces potets doivent intr. íablis aussi régulierement que le permettra la nafrre du terrain. On les espace suivant quion reut loniser serpe ou elair. One le semis doive dre lail en graines lomples ou legreres, en essences tracantes on pivotantes, la meilleure maniere diassurep le surris de lopération es diameublir profondement la lerpe du potet. Lee jemes planls sont beauceupl mieux grabantis rontre la sicheresse lorsque leurs rarines sienfoncent dans me trore menble que lorsqüelles trouvent une lerre tassée, que la chaleur. pinetre bien plus aisiment. Les grands travaux dr whoisement latsedepuis trente ans dans les terrains 


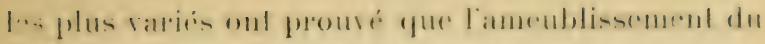
sol est. pour la plupart des semis, more eomblition nécessaipe de péussite.

On seme les graines lourdes, telles que glamels. chatiagnes, ete., dans des frous quion ourre a la hour ou an plantoir. Ces semences doivent ibl. it peine recomvertes dans les terrains forls, un pros daranlage dans bes forpes légions: on place dens graines au moins dans le mème trou.

Les graines resineuses veulent btre semées presque a theur de terre; il suffit quelles soient à l'abri du soleil et du vent. On oblient ce résultat pn les recourrant, is laide diun ràteau, d'une couche tris liegere de tere régétale. Les semis artificiels de sapin et dépicia réussissent rarement sur des sols découverts: on doit de prélérence employer le procridé des handes alternes ou des potets. Si le sol esl completement déourert, il fandra abriter le semis aree des branchages, quion enlivera apres que les jeunes plants auront bien pris.

In a mhoise en pin sylvestre des terrains garnis de bruyires en répandant la graine à la volé Inrsque la terre est rerourerte des dernières neiges du printemps. La font de la neige entrañe la semence rers lo sol, ly fixe et le jeune plant troure ¿̇ labri des bruyères les conditions nécessaires à sa régitation. Co profédé ésonomique ne peul ètre em- 
ployé yue dans des terrains assez profonds el garnide bruyères courtes. Si les bruyères sont hautes et épaisses, il faudra les brûler et semer deux ou trois ans après l'écobuage.

Les jeunes plants, surtout ceux de chène et de chataignier, demandent à ètre débarrassés des herbes qui entrarent leur croissance; ceux de hètre ef de sapin croissent mieux sous l'abri des plantes parasites, pourvu quils ne soient pas étouffés. Il faudrar donc parcourir les semis en les nettoyant des mauraises herbes, repiquer dans les places rides les plants trop nombreux ailleurs, et surtout les garantir arec soin des atteintes du bétail.

On a préservé des semis considérables, en faisant répandre par les gardes quelques poignées de plàtre yüils faisaient passer pour de larsenic. La craintr. de voir les bestiaux empoisonnés donnait aux bergers une vigilance quion ne pourait obtenir autrement.

Plantations. - Les plantations se font en automue ou au printemps. Les bois feuillus peurent chre indifféremment plantés dans les deux saisons : en pays de plaine. il est préférable de planter les résineux en automne; en montagne, on les plantera an printemps.

Le suceè diune plantation dépend beatucoup du rhoix des plants et des soins quion apporte a les extraire et à les mettre en terre. 


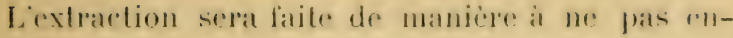
fommager les racines, qui doivent rester fraichesel garnies de chevelu; il ne faul pas rmployer dre planls qui ont crù sous un couvert epais : ils sonl labougris et languissants.

Guand on ne peut se procurer des plants de pepinieres, il faul choisir, dans les compes densemencement et sous les arbres réservés dans les taillis, les brins de semence les mieux renants; lextraction de res plants doit se laire par un temps pluvieux; si le: sol était désséché, on briserait toutes les l’acinrs. Les jeunes plants de chène doivent ètre arrachès ¿ la bèche.

L'extraction des plants d'essences résineuses demande plus de soin encore que celle des feuillus; leur reprise est plus difficile. Pour aroir un suces assure, il convient de les planter en mottes on par touffes; cest alors dans les pépinièes quil faul se procurer les jeunes sujets. Nous indifuerons plus loin la manière d'exécuter les plantations de cette nature.

Les plants doivent ètre garantis du froid et du soleil, et ne peuvent ètre longtemps conservés hor's de terre. Les radicelles sont formées de tissus jeunes et tendres, qui se dessèchent avec une grande facilité; quelques instants d'exposition au soleil suffisent pour les rendre impropres a remplir leur fonctions dab- 
sorption. Quand on transporte des plants, il faul donc aroir grand soin de les tenir à l'abri en recourrant les paniers de mousse fraiche ou d'une toile mouillée.

On ne se rend pas assez compte, en général, des précautions quil faut prendre pour assurer lat ro prise dun petit arbre, aussi les insuccés en matior de reboisement sont-ils très fréquents. On pourait en ériter beaucoup arec quelques soins faciles et peu coûteux.

Si, pour une raison ou lautre. on est obligni: de suspendre une plantation dont les sujets sonl arrachés, il faut les mettre en jauge, cest-à-dire les placer provisoirement dans des rigoles, à lombre, en recouvrant les racines d'une épaisse couche de bonne terre.

Les trous ou potets destincis a lat phatation doirent itre assez larges ef protonds pour que les racines du plant sy étalent completement: si elles sont l'op longues ou de forme irréguliere, on peut les laxroureir arec une serpe bien tranchante, en ayanl soin dy laisser assez de cherelu pour assurer la reprisc. Arant de placer le sujet, on rejette au fond du potet la terre végétale quion a extraite, on entoure les racines arec la main, et lon tasse ligèrement de manicre que la tige se tienne bien droite; on achève de remplir le trou, et l'on raflermit la terre en pressant arec le pied. 


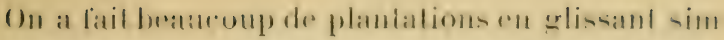
plement de jenurs plants daus l'ouverture partiques a la beche dans des gazons compacts. Il esl inulile dre dime que la plupart de ces repenplements onl mancfué il lant, pour que: le jemur planl reprentur.

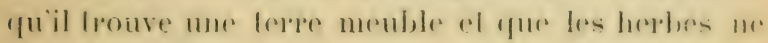
grenent pas sal croissance.

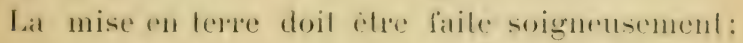
les jemes brins ne reprenant quautant que les rarimes sont complitement rntourées de terre amrublice la motte de gazon yui recourrait le potet doit ilre rejetée sur son bord méridional jour préserrer le plant de la forte ohaleur.

Plus les sujets sont àgés. plus il fiaut mettre dr. soin a les planter. On ne réussit a faire reprendre les résineux qui ont alleint lage de 6 à 8 ans yni les transplantant avec leur motle.

Ponr assurer la rémsite des plantalions de chime ou de chataignier, il nest pas de meilleur travai quine culture d̀ la houe; on débarrasse ainsi les plants des herbes qui les étouffent: leurs racines. trourant la terre ameublie, sont alor's dans les meilleures conditions pour se développer.

Cette façon peut ètre donnée d'une manière peut dispendieuse quand on a fait la plantation sur un sol préalablement cultivé pour ètre ensemencé cu

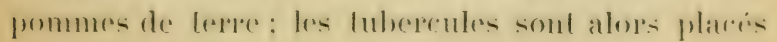


dans lintervalle des plants, qui profitent des facons qu'on donne au terrain.

Lespacement des plants varie suivant le but quion se propose d’atteindre; celui quion adopte généralement est diun metre dans tous les sens, il laut alors 10.000 trous par hectare; il est utile daligner régulièrement les plantations, on y pratique plus facilement les cultures nécessaires et l'on est moins exposé à écraser les jeunes plants.

Lorsquion emploie des plants de haute tige, cesti-dire din metre et au-dessus, les trous doivent ètre larges et profonds; il est utile de mettre autour des racines la meilleure terre régétale, c'est-à-dire celle qu'on trouve à la surface; en y mélangeant du terreau de feuilles, on assure la reprise. Il conrient toujours de battre légèrement la terre autour de la tige. Dans les terrains tres monilleux, il l'aut seulement culever la superficie du sol. lameublir et platcer le plant en recourrant les racines de bome terre végétale, quion butte assez haut.

Les plants de haute tige dessences feuillus doivent ètre retaillés proprement. On fait cette opéraration en coupant à la serpe ou au sécateur les branches principales, de manière à leur laísser seulement quelques boutons.

Les plantations de résineux par touffes sexécutent au moxen de plants extraits des pépinieres. On eit- 
leve a la beche les jeunes brins par molles de la crandeur diune brique; on transporte ces playuen dans un panier ou une brouelte, on les divise is la main de maniere a aroir des moltes contenanl dr. trois a six brins, on les place dans les lrous prepatrés a lavance, on garnit les interstices de forer of lon arrose, s'il est possible.

Le recepage consiste a couper arec une serpe bien tranchante chaque plant à s ou 3 centimètres au dessus du collet de la racine.

On ne recèpe que les plants de bois leuillus; les assences résineuses ne supportent pas cette opération. C'est après deux années de plantation et lorsque la reprise des brins est bien assurée que le pecepage produit les meilleurs résultats.

Lépoque la plus farorable pour receper esl moll. qui précede la mise en monrement de la sere.

Pépinières. - La créalion dune pépiniere es le plus sùr moyen d'obtenir des plants de bonne furalité, d'une extraction facile et d'une reprise presque assurée. Toute forèt bien soignée doit aroir sa pépinière, dont l'entretien est confié au garde du triage.

Pour établir une pépinière, il faut choisir un emplacement abrité, en plaine ou en pente douce; l'exposition du Yord doit ètre préférée pour les pépinières de résineux; si l'on peut disposer de quelques petits rours deau pour larposment. re 


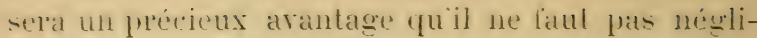
ger.

Le sul doit ètre défoncé à la bèche ou à la charlume ef romplitement nettoyé des mauraises herlues: "in jist protiter de cette première culture pour semer des ciréales ou des pommes de terre. Ciella

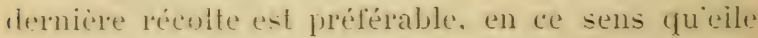
mécesite plus de laçons el yue la terre est complitrment ameublie par liarachage des tubereule-. Lameublissement ynion obtient par la culture ne -ullit pas pour rendre tous les sols propres a létilblissement dine pépinière lorestiere, il faut encor transformer le lerrain par des composts, afin yu il devienne assez liger et maniable pour you guisse

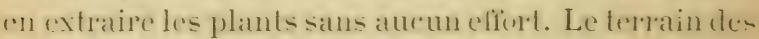

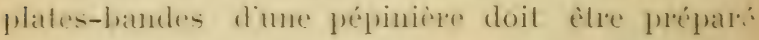

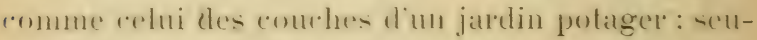
lement, an lieude lumicr, on emploie le terrean de

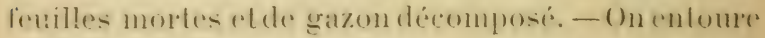
limplatement de la pépiniere deme haie ou diun losice. on divise le terrain en plates-bandes parr un chemin central anquel viendront aboutir perpendiculairement des sentier's sufhisants pour le passage diun homme : parallelement amx sentiers. on omre dans chargue plate-bande des rigoles de 20 à 2a centimetres de largeur et llune profoncleur de 25 a 30 ,

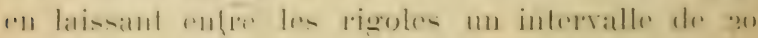




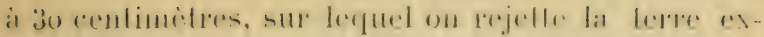
Iraite.

si lon veul employer le systime des plantations par toulfes, recommandé pour les résineux, les sil-

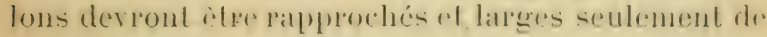
ji a centimetres; on remplira les sillons ainsi pratliqués diun bon terreaulu de leuilles mortes al de garzons décomposés quon aura dì préparer à lavance. on lasscra légerement la terre ef loun semera en recourrant très peu les graines; le semis doit otre tres ipais. On extrait plus tard les plants surabondants ot on les repique dans les espaces demeurés vides.

Les pépinizres destinées à lélère des résineux seront autant que possible établies dans la terre de hruyère. Ce terrain léger et facilement pénétrable anx racines lre's menues des jeunes plants est l. seni qui offre des garanties certaines de succes.

Sil ny a pas de terre de bruyère dans la localité. on la remplacera par un compost de feuilles mortes bien décomposées et de terreau léger.

Quelques soins que l'on mette à bien choisir la semence, à la répandre dans un sol conrenablement ameubli, on aura peu de chances de voir réussir.le semis, si on ne labrite pas contre les rents sees et lardeur du soleil. Les abris quon emploie varient suivant l'espèce de graines et anssi suirant le degré de déreloppement du semis. 
Pour les essences délicates (sapin, épicéa, mélèze, hètre), on recouvre les plates-bandes d'un paillis de mousse coupée ou d'herbages qu'on maintient pal' des branchages.

Plus tard, quand le semis est levé et déjà un peu fort, on le dégage de cette couverture: mais, comme il est encore trop faible pour résister aux coups de soleil, on lombrage au moyen declaies légères faites arec des genèts, des joncs ou des menues branches le sapin. Si le maniement de ces abris parait Irop compliqué, on peut y substituer des branches de genèts quion plante en traver's des plates-bandes, par lignes espacées denviron 1 mètre, de manière à ombrager légèrement le terrain ensemencé. Les abris indispensables pour quelques essences sont utiles pour toutes: aussi toute pépinière bien lenue doit-elle aroir son approvisionnement de mousse siche, de grenèts et de branchages.

Les semis doivent ètre, dans les premières années, soigneusement sarelés a la main et lorsyue le sol est frais.

Il conrient daroir dans la mème pépinière des plants de différents àges; on obtiendra ce résultat en ne semant qu une portion chaque année. L'extraction des plants s'opère à la bèche ou à la main; on regarnit de terreau les sillons d'où l'on a extrait les plank of lon y lail un noureau semis. Ourloques 
brins de haule lige doivent etre réservés dans une plate-bande spéciale.

On désigne sous le nom de pépinieres volanles les pépinières situées à proximité des lerrains à repeupler et dont la durée ne doit pas dépasser celle des pepenplements projetés. Les pépinières volantes sont ordinairement destinées à préparer des plants dessences résineuses, qui seront. transportés en motles sur lemplacement à garnir. - Pour quin lerrain soit propre à l'stablissement d’une pépinière de cefle espece, il faut quil soil à labri des rents desséchants du Yridi, sans itre trop ombragé. Le sol doit en ètre assez consistant pour ètre enlevé en mottes. Les terres siliceuses tris légères ne conviendraient pas pour l’établissement de pépiniores rolantes, parce qu'en enlevant les jeunes plants, on émietterait la terre qui les entoure. On prépare le lerrain par une culture donnée à l'automne qui précéde les semis; le labour à la bèche est préférable à tout autre mode de culture. Quand lhiver est passé, on régale le sol ameubli par les gelées et les pluies.

Les graines doivent ètre répandues à la main, et simplement recourertes au râteau. On sème en plein et assez serré. Quand on a lieu de craindre les coups de soleil et le hâle, on peut abriter les semis arec des branchages, quion relèvera dès que les jeunes plants auront pris un peu de foree. 
Pendint les grandes chalemes de léte, il rst fort utile d'ombrager un peu les plants au mojen de claies ou de branchages plantés rerticalement et formant des haies dans le sens de l'Est à l'Ouest. Si le terrain quion a choisi nest pas trop courert de mauraioss herbes, il pst inutile de sareler les semis. Cette opriration ameublirait trol, lo sol: d aillommles herbes soutiennent les pliuts ot les abritem lorsquion les enlève en mottes.

Les jeunes plants sont bons it employer dis qu ilont deux ans. On les extrait it la beche par mottes renfermant 4 ou jolants, et on les porte dans dis paniers ou des eivieres sur les places à repeuplere. On peut encole se servir pour la plantation en moltes de brins de trois ans: mais passe cel itgre ils sont trop, fortement emareinis pour itre arrarhis arec tout leur cherelu.

Boutures. - On regarnit les plares vides qui trouvent dans des fomets mouillenx au moyen de fontures de saules. de perppliers on daunes: on choisit de frefiremere les boulures provenant des gramels samles of des peupliers btrangers : celles de marceatu et de tremble ne ribssissent pas. Pour laire une lonture, on enupe bien franchement et en biseau un jet de deux ou trois ans dont on enlirie toutes les ramilles, on y conserve senlement trois ou quatre bourgeons et on le réduit a une longueur 


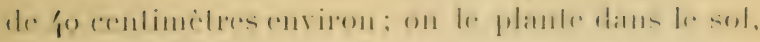

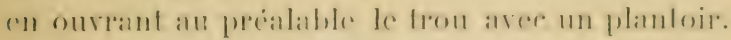

On domne le nom de mureolles a des lumbures fume lon fail an conchant dans le sol, mais satis les séparer de la sonche mere, des brins maintemus

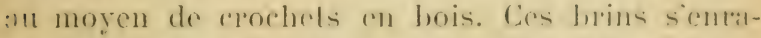

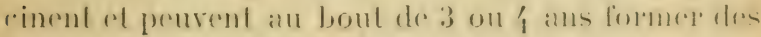
individus isolés. Si les brins quion veul matreofler.

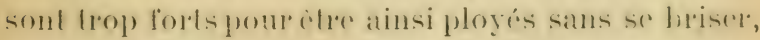
on jeul les entailler a moitie ; on les courbe Ientrmenl et on les fixe au sol par des fourehes solidrment enfoncées. Liextrémité des branches ainsi rouldres doit sortir de terre ef etre redressie arer des mottes de gazon. Il faut avoir soin de ne conlserrer sur la souche mère que les brins sommis an mareottage, car reux quon garderait dans leur situation naturelle absorlseraient la plus grame - partie de la sire, qui continuerait it sy prorter an lieu de se diriger sur les marcottes: 


\section{CHAPITPE X}

\section{TRAVAUX DIVERS}

Assaivissemext. - Tracé des fossés. - Régalage des terres. - Fossés de cloture. - Marchepied. - Dimensions. - Sources. - Ravins. - Barrages. - Con-olidation des berges. - Taille des réserives. Règles principales. - Elagage rez-tronc. - Emplui lu coaltar. - Branches gourmandes. - Taille des balireaux. - Viabrlité. - Lignes de coupes. - Sentiers interdits. - Nettoiement des chemins. - Curage des fossés bordiers. etc.

Assainissement. - Le's préposés sont sourent (hargés de diriger les ourriers qui ourrent les lossés d'assainissement : il niest pas hors de propos de donner quelques indications sur la manière dexécuter ces traviux. - Le but quion se propose étant. de laciliter lécoulement des eaux dont le séjour oceasionme une humidite nuisible, les fossés doivent Chre dirigés de manière à déboucher dans des ruisseatux, rivieres ou ilangs, ou elles trouvent une issur naturelle; si lon ne peul atteindre ce résultal, on les diverse dans les grands lossés de périmètre, ou elles sévaporent plus rapidement que dans lintérieur du bois: dans tous les cas, il faut ériter 
dimonder les fomds riverains. ()n prol, la plus souvent, tracer les fossés sans faire de nivellement. Quand on examine les points où l'eau séjourne le plus longtemps et la direction qu'elle prend pour s'écouler, on voit de suite le tracé des fossés à ourrir.

Les fossés doivent etre tracés au cordeau; leurs dimensions sont variables et se modifient suivant la nature du terrain ol la quantité deau is farre ceouler. Les terres extraites ne seront pas releveres sur les bords, ce fui produirail un bourrelel qui s'oppose à l'assèchement des parties voisines; cllu doivent ètre régalées sur les parties les plus basse's du sol.

Liouverture diune rigole suffit souvent pour assainir certaines parties mouilleuses garnies de jones ot d'herbes marécageuses; pour assécher les mares plus profondes, il est nécessaire d'ourrir sur le point où s'écoule naturellement le trop-plein une tranchée. de la profondeur de la mare.

Dans les terrains inclinés, les fossés ne seront pas dirigés dans le sens de la pente, mais obliquement ef en zigzags, de manière à éviter les mavinements el à recueillir le plus d'eau possible.

Les fossés de clòture derront ètre tracés en ligne droite, d'angle en angle; leurs parois seront bien régulières et la terre derra ètre relevée du côté du bois; on laissera un petit marchepied de 15 à 20 centi- 


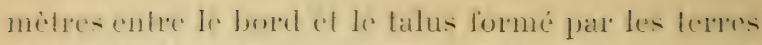
ainsi relerées, afin quelles ne s'éboulent pas; un ménagera de temps en temps une ouverture dans ce talus. afin que les caux des parties roisines puissent serouler dans lí lossé. Les dimensions des fossés de ritmesont ordinairement de 2 mitres dinurefunc, a metre de profondeur au fond. et so rentimètres de largeur.

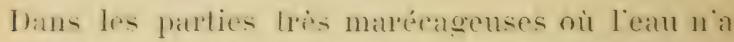
au-un écoulement, on ourrirar une sripe de fossis paralleles: la terre qui en proviendral sera rejetre sur les bords de manière à former une banquetle derreantelesusdu niveau de léau. Les plantations l'ammes de saules of de peupliers réussissent tries lifen sur dessols ainsi prépaless: il laul seulemenl employer des brins un pen forts ou des boutures.

Les sources qui existent dans l'interiene ou sur

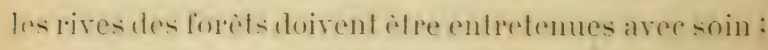
-i rlles sont dans linterienr des massils, on résor-

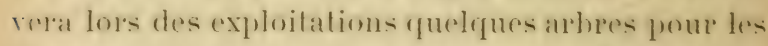
abriter, on en plantera au besoin, on facilitera liconlement de lean en nefloyant le fossi de décharger.

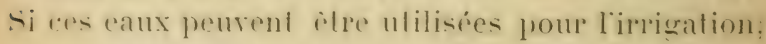
on enolitiendra des résultals smplemanls.

Les eaux qui coulen! sur les rersanls col penls rapide, apres les orages el la fonte des neiges, raiinent lo sol ef reusent des gorges qui deriennent 


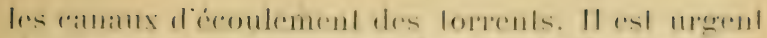
de prérenir res ravimements, anssi dresastremx pour les monlagnes ou ils se produisent que pour les rallées qui recoirent les sables el les galets emportés par les caux. Pour cela il faut arriber lo comrs des forrents par des barrages rustigues blahlis it lorigine des ravins. Ces barrages se enostruisent dome maniepe simple ef feconomigue, on moyen de lascines dre salules quion place an latrels des ravins on les fixanl aree de forts piqueds; on laisse sortir de terre les restrémités des brindilles, qui premuent bientòt racini el forment de réribables haies rivantes. La vilesse des caux, amortie pal eres olstacles, n’étant plus assez grande pour affouiller le sol el entrainel les galels el les sables arpachés aux parlies supérieures, res matériatix sre déposent en amont des barrages, ol le ravin se comble pen à pell.

Les pelits barrages en lisernes, tris bons pour prevenir la création des brosions nowvelles of pour mpecher de sarcoitre colles qui existent depuis yuelque temps, ne suffisent pas sil s'agit dardèter. les progrès de ravins déjà profonds; dans ce ras, on est oblige détablir des barrages en pierres ef en maçomerie. Ces traraux de construction, qui se rattachent à lart de lingénieur, sont toujours arcompagnés de travaux lorestiers destinés à en compléler les effets. Ainsi, à mesure que les atterrisse- 
ments se forment en amont des barrages, on les garnit de plantations de peupliers, de saules, d'érables, de frènes et autres essences propres aux terrains frais. Les talus rapides des gorges au fond desquelles se trouve le lit du torrent doivent ètre lobjet de soins tout spéciaux, car les eaux et le passage des troupeaux désagrègent constamment leur surface. On interdira dabord l'accès de ces talus aux troupeaux, puis on ýtablira une série de sen-

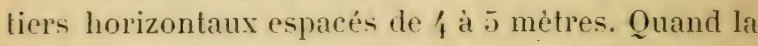
pente, ainsi divisée en une succession de bandes séparées par des sentiers à peu près parallèles, sera devenue aisément accessible dans toutes ses parties, on soccupera de garnir la surface des bandes aver des essences appropriées au climat et au sol. Aux grandes altitudes on emploiera les mélèzes, les épicéas, mèlés aux aunes et aux saules; dans la zonı moyenne, les frènes, les hètres, les sapins, les pins sylvestres, laricios et d'Autriche, et plus bas, les rhines, les sycomores, les ormes, ete. Si quelques parties des talus menacent de s'ébouler, on les consolidera au moyen de forts piquets réunis par des rlayomnages, et l'on y sèmera des plantes diune croissance rapide. Ces diverses opérations, combinées suivant la nature et la disposition du terrain, permetlront de ramener à l'état de ruisseaux paisibles les torrents les plus dangereux. 
Taille des réserves. - Le's cahiers des chargres preserivenl quelquefois aux adjudicalaires déldaucher les réserves; il importe que les préposés puissent diriger cefte opération; il n importe pas moins 'füils sachent lexécuter eux-mènes, de manimer is pouroir, dans leurs tournées, rectifier ou dirigrer la rroissance des arbres qui prenneut une mauraise: forme. Nous indiquerons done quelques règles de la laille des arbres forestiers, en prérenant toutefois rue les limites de cet ourrage ne permettent pas dentrer dans tous les développements que nécessite cette étude.

On pousse un arbre à croitre en hauteur en le débarrassant des branches basses, mais il faut toujour's lui conserver une tète suffisamment garnie. On force un arbre à śétaler en supprimant sa flèche. On arrite laccroissement diune branche en pinçant lixllémité des rameaux ou en lés coupant.

Les cicatrices produites par la section des grosses branches se terment difficilement et occasionnent des plaies qui diminuent la valeur des tiges : aussi doit-on éviter ces opérations. On se bornera à raccourcir les branches quion reut supprimer, en y laissant toujours quelques rameaux pour y entretenir la vie. - Si on est obligé denlever de vieux chicots ou des branches mortes, il faudra, quelques jours après labatage. enduire la section arer du 
goudron de gaz oul comllar. Lat Laille serlal toujours laite arec un instrument bien franchant el de bas en haut jour ne pas déchirer l'écorce; les branches doirent ètre coupées rez-tronc; cette recommandation est expresso. Plus la surlace de la sertion sera nette et mieux se fiera le recouriemenl. Ponr yue l'élaggage soit bien lait, la plaie doit etre mine et se raccorder sans ancun ressaut arec lecorlos du sujet. Toute saille est un obstacle au rapprochement des deux bourrelets yui doivent se souder puir lesmer la blessure. Les branches gommandes yui si produisent sur le tronc des chènes réservés dans les laillis doirent etre coupres dans les 3 on fannés yni surent lexploitation. Les baliveaux qui tene?ent

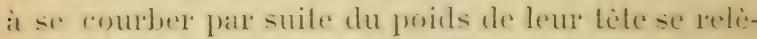

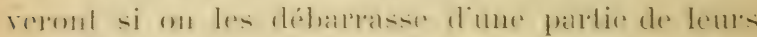

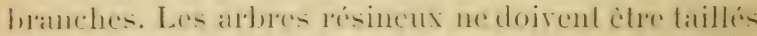
yue dans des cas exceptionnels.

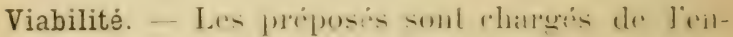
frelien des lignes de coupes ot daménagement: ils liennent ces laies el tranchees libres en élaguanl les brancles qui les obstruent. Cet élagrage se fait a la serpe ou plus facilement avec un sabre d'abatage. Les bois quien proviennent appartiennent aux gardes.

Lorspüil y a lieu de labre relerer les ligiaes de coupes. travail qui est sourent imposé à lientrepre-

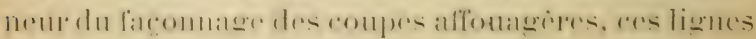




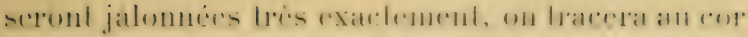
deau les drux rólés parrallèles. puis a laide dimm.

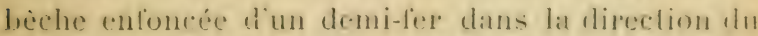
rordean, les lerres seront relevées sur laxe de ma-

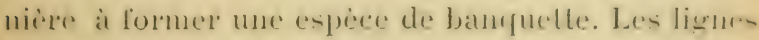

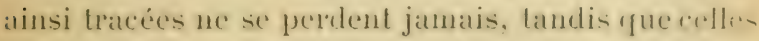

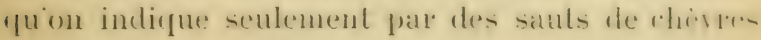
sont souvent difficiles à retrouver.

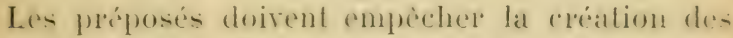
sontiers dans les jeunes compes on dans les somis:

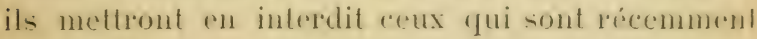
lormés.

Lorsque la traite des bois seral terminée, ils interdirout aux voitures les chemins de vidange des rompes uséesentelevant les barriéres. s"il en exisle:

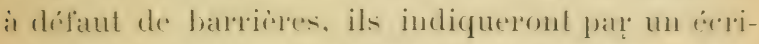
lean yue le passane est interdit ambetes desomme.

Afin dempecher les chemins non empierexes dr. sobstruer par la regétation des herbes et des roners, los gardes derront les laire nettoyer tous les ans au moins. Ils peurent utiliser ces chemins comme pàtures, mais à la condition que les bètes scront tenues à la main ou attachées à des piquets. Ce dernicr système est tris bon, parce que les animaux, restant plus longlemps au mème point. broutent liherbe de plus près et favorisent ainsi lir formation diune pelouse compacte. 
Les fossés bordiers seront tenus libres et curés à vif fond, afin que les eaux y trourent toujours un ćcoulement facile. Arec des fossés bien nettoyés, la chaussée reste presque toujours solide, tandis qu elle se défonce aisément si l'eau des fossés s̈inliltre dans les terres sur lesquelles elle est assise.

Les travaux que nous renons dindiquer sont loin de comprendre tous ceux que les gardes peurent exécuter ou faire exécuter; nous arons dù nous restreindre, notamment en ce qui concerne les semis ot les plantations, aux renseignements relatifs aux essences les plus répandues. Pour plus de détails. nous renverrons aux ouvrages spéciaux, et notamment à l'Art de planter 1, de M. de Manteuffel. et à lexcellent traité sur l'Élagage 2, de M. le comte des Cars.

Les preposés de loute ratégoric doivent tout leme lemps à leur service. Celui qui n est pas employé en tournées doit l'ètre en travaux d'amélioration. La surveillance nen est pas moins efficace et la forèt profite grandement des travaux, quelque peu importants quils paraissent dabord, sils sont conti-

${ }^{1}$ L'Art de planter, traité sur l'art délever en pépinière et de planter les arbres forestiers: traduit de l'allemand, 1 vol. avec 16 vignettes, prix 2 fr.

2 L'Élagage des arbres, traité pratique de lart de diriger et de conserver les arlires, par le comte le Cars; 1 vol. $7^{2}$ onarures. prix 1 fi: 
més aree perseveranee. Des gralifiralions spredales sont accordées par ladministration aux gardes yni ont exécute des travaux d’amélioration dans lus forets domaniales. Les societés dagriculture decernent aussi des récompenses aux gardes des communc's ct des particuliers a raison des soins qu ils apportenl i tenir en bon état les forèts qui leur sont conliés.

Ce nest pas tout, en effet, pour un préposé, qur d'etre actile rigilant, de préserver son triage contre les dévastations des délinquants, il fant cneore laméliorer; c'est à quoi il peut parvenir par des travaux qui sont généralement d'une exécution facile. T)es semis opérés avec les graines récoltées daus les bois. des plantalions, des boutures, des marcoltages peurent ère fails par un homme scul, à loisir el sans aucune dépense. La création des pépinières. lentretien des travaux d'assainissement demandent de plus grands efforts, mais sont d'une utilité si évidente, quim bon forestier ne doit jamais regretter le temps quil y emploịe. 


\section{CHAPITRE NI \\ OPÉRATIONS DES COUPES}

Arpentare- - Arbres de limiles - Dégagement des lignes. - Uutils dabatage jalons pipuels. - Ceintu. rage des arbres de limile. - Entretien des lignes. M.mpelages- - Martelages en réserve, en délirranee. - Prejaration des rireses. - Empreintes. - Criés. E-truatioss. - Marifue les arbres abandonnes. Crices. - Dinonbrements. - Cubage des bois épuarris, au rolume réel. au 5 , au 6 , au 14 . - Tarif. Rérolemexts - But de loprération. - Ceinturage des reserves. - Virés. - Griflage. - Alpels. - Fausses marques. - Récolement.de souches.

Arpentages. - Nous arons ru dans les clalp. ri et vin que les rompes de taillis of relles dimmeliome

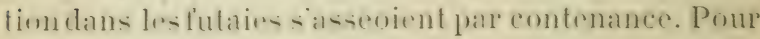

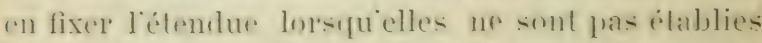
-ne lo forpain far lamenagement. il faul chatue

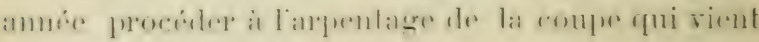
en tour diexploitation.

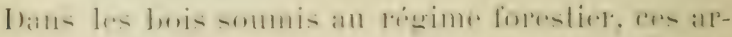
pentages sont faits par les chels de eantommemmut. Dans les buis des particuliers, ils sont late par des giomitres arpentrurs chnisis par les propricitarce :

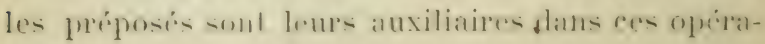
tions. 


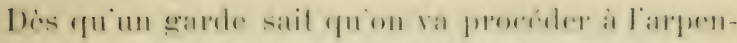

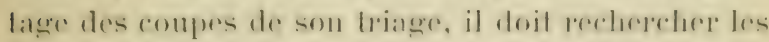

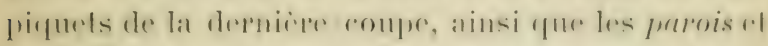
corniers. On appelle parois des arberes qui se loon-

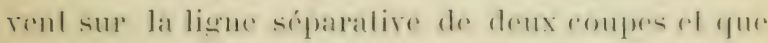

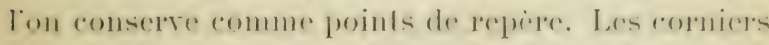
sont des arberes qui se trourent a langele de deux lignes séparatires. Lesparois sont marrutes aux eopjus du coté de la enupe qüils délimitent. Las contuiers sunt aussi marrués au corps, dans la direction des lignes dont ils oceupent le point de jonction. Quand il aura recommu toute la ligue, le garde planterades jalons contre les piquets ot les arbres de limile. Si la tranchée est trop longue pour quine des rxtrimités puisse être aperçue de lautre, il placera des jalons intermédiaires, de maniere qüils se tronrent sur la ligne droite qui joint les deux piquets cntre eux.

Un garde doit connaitre la position quoecupera la coupe a asseoir; il préparera le travail de larpenteur en dégageant autant que possible le périmitre de cette coupe ; il élaguera les brins trainants, débarrassera les bornes, sil en existe, des herbes et ronces qui les cachent; si la ligne est obstrue par des bois de la coupe en exploitation, il obligera les adjudicataires à les faire immédiatement enlever.

Lorsque lagent chargé de larpentage aura incli- 
qué le jour et liheure de son arrivée, les gardes qui sont désignés pour l'assister s'entendront pour ètre munis des instruments nécessaires; il est d'usage que le garde du triage soit chargé de porter une llache, sil en est besoin ; les gardes roisins se muniront alors de bonnes serpes. Si l'on opere dans des: taillis un peu forts ou dans des futaies, il sera bon d'aroir deux ou plusieurs haches. Un sabre diabalage ou briquet est tris commode pour parer la ligne. mnlever les brindilles et les leuillages qui masquent les jalons. Tous ces instruments doivent ìre bien tranchants et solitement emmanchés.

Les jalons seront, autant que possible, choisis parmi les brins les plus droits: les deux extremités seront taillées en pointe.

Les pirquets destinés at marquer les extrémités des lignes doirent aroir au moins 15 à 20 centimètres de rirconférence. leur longueur él proportiommin à la profondeur dn sol, la tète est taillée carrément pour porter lempreinte du marteau de larpenteur. ils sont enfoncés dans le sol aree le talon de la harhe. Ces piquets doivent, autant que possible, ètre en chène, chàtaignier ou autres bois durs: ceux de bois blane, de charme et de hitre se pourrisent avant l'époque du récolement.

Quand larpentage est terminé, le garde du triage intoure les parois et les corniers diun lim, de ma- 


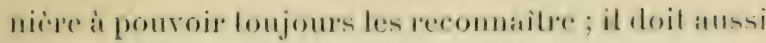
sassurer de la presence des piqfuels, quil remplare. sils viennent à che brisés ou enlevés. Quand la compe est divisée en plusiemes lots, il est bon d’indiquer le numéro de chacun dioux par des entailles liaites sur les arbres de limile.

Les lignes darpentage dans les bois taillis doivent, autant que possible, ère conservées mime après le pécolement, an il est tomjours utile de pouvoir distinguer au juste lemplarement de eharue eoupe.

Les gardes assureronl la conservation de ers lignes en ślaguant, surtout dans les années yui suivent lexploitation, les brins qui les obstruent; ils remplaceront les piquets soit par de petits fossés, soit par dautres signes, suivant la nature du sol.

Dans les bois de l'État, où les coupes sont séparées par des tranchées et des laies sommières, les gardes sont chargés de l'entretien de ces lignes; les herbes et les produits de l'élagage leur appartiennent. (Décision ministérielle du 10 novembre 1835.)

Martelages. - J.es balivages ou martelages, car dans la pratique ces deux expressions semploienl pour désigner une seule et mème opération, sont laits par les agents forestiers arec lassistance des 
próposés: ceux-ci sont spécialement chargés de la marrue les arlures a réerver ou à abandonner

Il y a deux especes de martelages: ceux en résprev, dans lesquels les arlores qui doirent itre consorvés recoivent lempreinte du martean: ceix en dislipunce. wit lon appose an contraire cette cal:preinte sur les arbres abandonnés.

Dans certaines compers, ou les arbres ne sont las assez lorts pour supporter linpreinte du marteau, lat marque or fait au moyen de grilfes ou mème par la drighation des dinensions ou essences derarbres.

Les roupessont dites en rérere on en télivance. suivant que le griffater ou la désignation des arbers prorte sur cenx it riserrer ou it abandommer.

Les martelages or fout pas virés ; les gardes, an

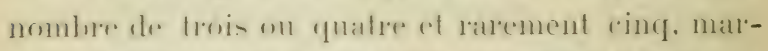
rheml ensemble el aulant que possible de front it 7 ou 8 metres dintervalle, ehoisissant, sous la directiondes agents qui les snivent. les arthres a marquer. et frappant du marteau ou griffant cenx qui lan sont désignés.

Pour lat iliter a travail. Ir- viress Inivent en grinéal itre proparese at lavanee. Ared effet, le garde du triage devra partager les coupes a marteler en un eertain mombere de handes a peu pris paralli les. an moyen de tracés qui sondiquent soit far des 


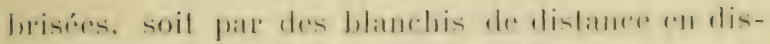
lance.

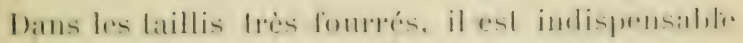

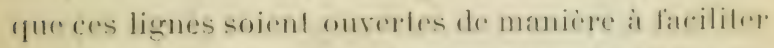

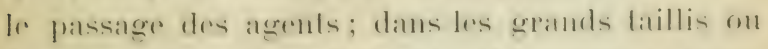

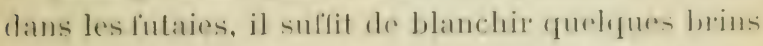
fous les 7 a 8 molres pour indiefuer la direetion des virées: celles-ei doivent elde dautant plus étroites

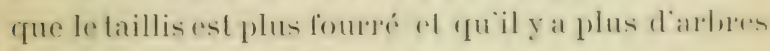
it martuer. Dans les Laillis de vingl-reinf ans moyennement garnis, elles peuvent avoir de so at 30 molres de largeur.

si le sol est en plaine ou en pente doure, les virées doirent ère toujours daus le sens de la plus grande longueur de la coupe. afin d'éviter de mulplier les mourements de conversion qui occasionnent une perte de lemps; dans les terrains fort inclimés, elles doirent itue dirigées en trarers de la pente.

Le martelage sopérant toujours sous la surveillance des agents, nous navons it domner ancune indication sur la direction de ces opérations. relatirement a lappliration des aménagements ou des riegles de la culture des bois ; nous nous occuperons seulement de la partie matérielle et pratique, pour domer quelques conseils sur les précautions que les préposés doivent prendre.

Les marteaux doivent elre sourent examinés, afin 
पue la monture soil toujours en bon état et le tranchant aiguisé ; un marteau qui n'est pas en main ou qui ne coupe paś fait perdre beaucoup de temps et ne donne pas de bonnes empreintes. La marque se léra toujour's du côté de la virée suirante.

Les blanchis, quel que soit le mode de martelage. doirent ètre assez larges et attaquer toujours l'anbier sur lequel l'empreinte doit ètre apposíc: unc rmpreinte sur lécorce peut s'enlever ou se détruire aisément.

Les gardes auront la précaution de ne pas laisser le morceau d'écorce enlevé sur le blanchis adhérant rncore à l'arbre ; en temps de sìve, cet éclat est susceptible de se ressouder sur la marque, et les adjudicataires ont profité quelquefois de cette circonstance pour modifier le martelage à leur arantage.

Dans les martelages en réserve, lempreinte est apposée à la patte les baliveaux portent une seule empreinte, les modernes en ont deux, les anriens une seule. Relativement aux modernes, il faut avoir soin que les deux blanchis ne se confondent las en un seul; ils doirent itre laits sur le mime côté de l’arbre, mais séparés.

Dans les eoupes en délisranee, lempreinte dı marleau s'applique sur le corps et la racine des arbres abandonnés; les blanchis doivent etre lai'giment faits, lempreinte fortement apposies. 


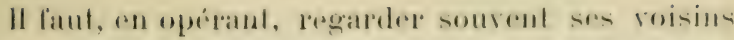
pour mather aver ensemble el espacer convenablement les arbres; avant de les marteler, il faut en examiner le corps et la tele. Un garde qui marrque comme réscrve un arbre creux ou mont en cime s'attire de justes reproches.

L'essence et la qualité de larbre doivent ètre críes d'unc manière netle et accentuée, suivant les indications qui sont données au commencement du ma1telage par les agents opérateurs. Il faut encore avoir soin dappeler en tournant la tète rers less agents, ol de bien prononcer les noms des essences; renx dr hìtre, frène, chêne, sont assiez faciles ì rontoncipe quand ils ne sont pas bien articulés. Les grardes doivent éviter d'appeler ensemble, car la confusion des sons occasionne sourent des erreurs dans le pointage.

Estimations. -..- Les eslimalions se font en mème temps que le martelage dans les coupes marqués en délivrance et dans beaucoup de coupes en réserve; dans les autres, elles se font immédiatement après.

Les gardes, suivant les virées du martelage, alppellent les arbres abandonnés, en indiquant l'essenre et la dimension. Les indications à donner varient. d'ailleurs, suirant la nature des exploitations. Dans les compes de putaies, ou les arbres ont des desti- 
nations dillerentes, dapres leur grosseur et leme hantenr. on appelle la circonférence mesurée is hantrur athomme, la hauteur du trone justuan point wit il cesse detre propre at dommer des hois de ser'rice ef de travail, ef le rolume en steres du houpjore : wette derniere indication se lait a rue diril.

Dans les eoupes de taillis sous futaie, on indiefue sentement la cresserur et la hauleur du trone: le volume des branches ost estine par les agents suivant des moyermes quils obtiement it part. Les arbes abandommes dans les compes marqués en

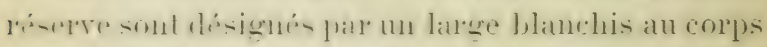
pour exiter unuls soient eslimés leux fois. Ces blanchis doirent etre apparents et loujours marqués du cole de la rirée suirante, afin que les gardes atimalents puisent, en revenant, sassmer quils n'ont oublié aucun arbre.

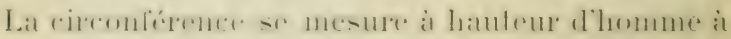
la rhaine metripue. Mais lis gardes na alpellent pas

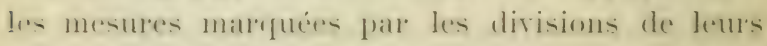
chaines, car les larils sont ordinatement calenles

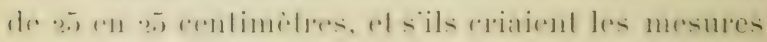
dre lat rhaine, les estimateurs qui tiement les calephins

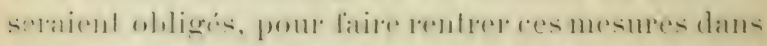

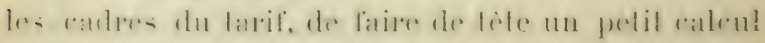

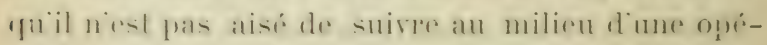

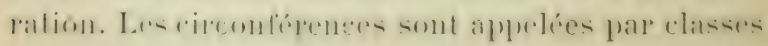




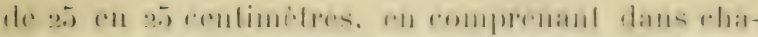

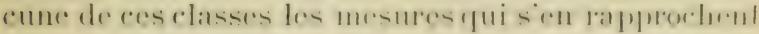

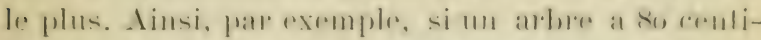

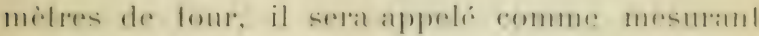

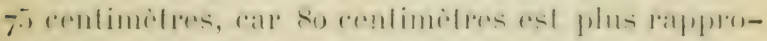

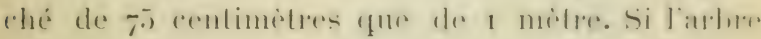

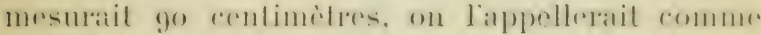
ayant 1 mille. Il sélablil aimsi mor rompensalim gui diminue les erreurs.

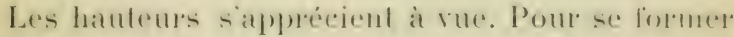
16 eomp diril, les gardes pen exprimombis lineml

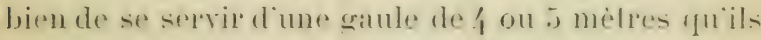

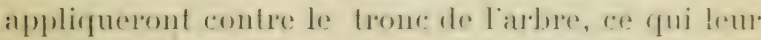
permettra dien éraluer exilctement la hauteur.

Lestimation du taillis se fait par apprération a vue d'ceil ou par places d'essai.

Les gardes doirent, pendant la durére deserploilations, estimer le rolume des houppiers de gurel-

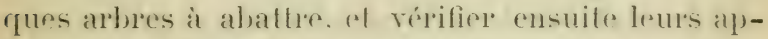
préciations aprés le facommage de ces hois: ils doirent aussi mesurer la hauteur des arlores abaltus pour se pendre compte de lexactitude de lour ivaluation arant labatage; ils tiendent un comple exact des produits réels de chaque coupe.

Dans les coupes adjugées, il leur sera lacile te commaitre ce produit, soit daprès le registre des lartours, soit diaprés lo dénombrement qui est 
ordinairement lait par ladjudicataire. Ils distingueront les produits en bois de feu, quartiers ou rondins, bois à charbon, fagots, bourrées, etc.

Dans les coupes affouagères, le dénombrement auquel il est procédé, arant le partage des bois entre les affouagistes, permet de connaitre très exactement le produit en matière. Un garde doit pouroir, it la fin de chaque exploitation, indiquer d'une manière précise le nombre de steres de bois de leu ou de charbon, de cents de fagots ou de bourrées, de perches, etc., qu'elle a produit.

Il doit aussi connaitre les prix du façonnage du stere de bois de feu ou de charbon, du cent de fagots, de bourrées, de perches, etc.. celui de l'abatage des arbres et aussile prix de vente sur feuille des divers produits, siils sont lirrés en forèt aux acquéreurs.

Tous ces renseignements sont indispensables aux agents pour pouvoir rérifier les estimations des années précédentes, ef baser leurs évaluations pour les coupes à estimer. En les recueillant, les gardes apprennent ì se rendre compte du produit des exploitations et se forment aux estimations. que la pratique apprend mieux que le meilleur lirre.

Dans quelques circonslances, les préposés sout obligés de faire eux-mèmes l'estimation de cerlains bois, notamment des chablis et volis quils reconnaissent dans leurs tournées: il est utile qưils 
sachent de qu'elle maniere on procede; il est. an reste, indispensable qu'un forestier connaisse lm éléments des opérations quil roit journellement pratiquer. Tous indiquerons donc les diverses nuthodes employées pour le cubage des brois, en accompagnant ces notions de quelques exemples destinis à en faciliter lapplication.

On obtient le volume din arthre irfuarri on multipliant le chiffre de la longrueur par te produit dec chiffres qui expriment la dimension des côtés do l'équarrissage.

Léquarrissage so mesure sur le miliru de la longueur. La nesure se prend de centimetre en centimètre; on néglige les fractions.

Les longueurs se mesurent en décimetres, les fractions de décimètres sont négligées.

Il est de rigle que dans les cubager faits pour lestimation des bois en forèt. on ne consirlère que des pièces dont les còtés d'équarrissage sont égaux; car cest à cette forme quion ramene tous les bois. sauf à les débiter plus tard en pièces à faces inégales.

Soit, par exemple, un arbse de $10^{\mathrm{m}}$, o.j de longueru', ayant un équarrissage de $0^{\mathrm{m}}, 457$ au milieu de la longueur : pour obtenir le rolume, on multipliera d'abord par lui-mème le còté d'éruarrissage qui est

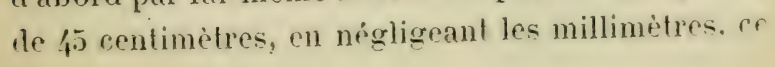


proluit $0.45 \% 0.45=0.2025$ sera ensule multiplié par la longrueur, 10 mietres, car on négrlige encope les 5 centimetres excédants, on aura ains $0,2025 \times 10$ mètres $=2^{\mathrm{m}}, 025$.

Le volume diun arbre abatlu, mais non equarri. $\therefore$ olotient de plusieurs manieres differentes, suivant les halitudes du commerce dans le pays. Ces dilierents systemes sont le cubage: $1^{\circ}$ au rolume réel ou comme bois rond; $2^{\circ}$ an $5^{\circ}$ déduit; $3^{\circ}$ au $6^{\circ}$ déduit; $4^{\circ}$ au $1 / 4$ sains réduction.

Le "ubage comme bois rond est peu employé dans la pratique commerciale: il derrait cependant etre relusirement adopte par les forestiers, car il donne seul le véritable rolume; mais il a été systématiquement repousie far les marrhands de bois. yui lui prélërent les méthodes de cubagge qui font conmaitre, non le volume réel du hois rendu, mais te rulume des pieces quion en pourra tirer.

Cest ainsi quion a sé conduit a adopter le mole

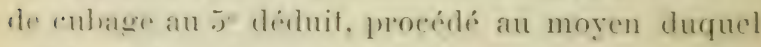
sobfirnt lo volume de la piece quion tirepa diun irlere an grume, quand on lanra éfuarri a vive arète.

Pour cuber un arbre au je deduit. on prend le 5 d. la circonférence mesurée sur le milieu de la longueur. on multiplie ce 5 par lui-meme, et le produit mulliplic par la longueur donne le cube eherehé. La 


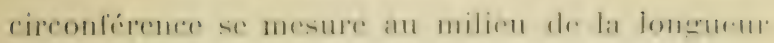

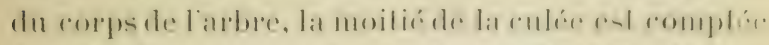

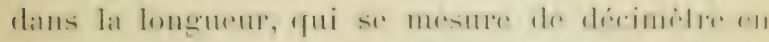

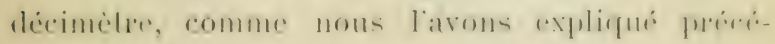
demment.

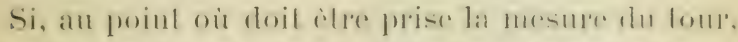

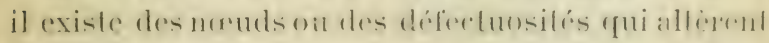
les verilables dimensions, lo mosurage soral lail 111 peu à còté.

Linsi, soit manteredeg milese de longuemr, dont

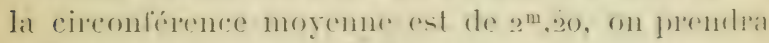
le je de $2^{m}, 20$, soit 任 centimelres, on multiplir prat 作 ce reui donne $0^{m}, 1936$; on mulliplie es produit par 9 , et le nombre oblem représente le volume

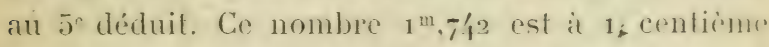
près égal à la moilié du volume caleule comme. bois rond.

Le cubage au je déduit donne un volumo infórieur à celui quion obtient après l’éruarrissagge; il serail très arantageux pour larequéreur si le prix du mélw cube nétait angmenté en proporlion de cel avanlate: : il est lacile de roir, dapres la comparaison des résultats de ce mode de cubage el de celui comme bois rond, que, si le mètre cube mesuré commie bois rond raut 30 francs, il derra valoir près de 60 francs au $5^{\circ}$ déduit.

Pour cuber au $6^{\circ}$ déduit, l'om prend le $6^{\circ}$ de le 
circonlérence moyenne, on déduit ce $6^{\circ}$ de la circonférence, on prend le 1 \& du reste. on le multiplie par lui-mème, et le produit multiplié par la longueur domne le cube cherché : ainsi, reprenant liexemple ci-dessus, le $6^{\mathrm{e}}$ de $2^{\mathrm{m}}, 20$. chiffre de la circonférence movenne, est de 36 centimètres, on le retranche de $2^{\mathrm{m}} \cdot 20$. le reste est de $1^{\mathrm{m}}, 8$, on prend le quart qui est de 16 centimetres. on multiplie ce nombre par lni-mème, ce qui donne $0^{\mathrm{m}} .2116$, on multiplie ce produit par la hauteur g. el le résultat est le cube cherché, $1^{\mathrm{m}}, 90 \%$.

Enfin, pour cuber du quart sans déduction, on prend le quart de la circonférence moyenne, on multiplie re nombre par lui-mème, et le produit multiplic par la hauteur donne le cube cherche. Pour appliquer ce systeme au cubage d un arbre de $2^{m} \cdot 20$ de circonférence moyenne sur 9 mètres de hauteur, nous prendrons le quart de $2^{\mathrm{m}}, 20$, qui est de í rentimetres: ce nombre, multiplié par lui-mème, tromme 0.2916, qui, multiplie par 9, chiffre de la liauteur, domnera pour résultat $2^{\mathrm{m}}, 62$.

On evite les calculs quentrainent ces différents cubages par lemploi de tarifs ou comples faits, dont il existe un grand nombre. Chacum de ces tarif's est précédé diune petite instruction qui indique la manière de l'employer.

Dans l'impossibilite ou nous sommes de joindre 
it ce rolume des lables de cubage complides pouvant servir pour estimer les bois romels el les bois équarris, les arbres abaltus el exux emene sur pied, nous ne pourons mieux litire que de renvoyer nos lecteurs aux larifs de cubage do .I. Comrsaud (1). Tous insérons toutefois, à la fin decel ouvrage, un pelit taril, dressé de maniere il fouvoir servir pour calculer le volume des arbres aballus, que les préposés sont sourent obligés d'estimer sans le concours de leurs chefs.

Ce tarif n’est bon que pour les estimalions des bois abaltus, mais non éfuarris. Il ne pourrait servir pour préparer lestimation dune coupe de bois encore sur pied. Lorsque les préposés auront à faire des calculs de celle espèce, ils derront aroir soin de se munir de tarifs spéciaux.

Récolements. - Le récolement est lopération rui a pour but de rérifier si les adjudicalaires ou les entrepreneurs des coupes ont satisfait à toutes les obligations qui leur sont imposées et sils nont exploité que les arbres compris dans l'adjudication.

C'est au récolement que les déficits de réserres sont reconnus par le moyen d'un comptage général. C'est aussi au récolement quon relève les rices

(1) Traite de cubage, de M. Goursaud, inspecteur des lorêts. Paris, librairie Rothschild, pr. 1,50. 
dexploitation et en général toutes les contrarentions commises par les adjudicatares ou leurs ourrier's. (qui nont pas été constatées pendant la durée des exploitations.

Ces opépations ne peurent ètre laites que par les açents. Les préposés sont leurs auxiliaires et procident sous leur direction au comptage des arbres de réserve ou des souches exploitées.

Pour préparer le trarail des récolements, les adjudirataires sont obligés.par le cahier des charges, do faire ceindre diu lien apparent tous les arbres riservés. Les préposés. prévenus à lavance du jour nu se fera le récolement de charjue coupe, doivent veiller à ce que celte obligation soit exécutée.

1ls doivent en outre rechercher les pirquets ef arbres de limite de la coupe puis en jalomner le pépimetre. afin que des arbres des coupes voisines ne soicnt pas compris parmi erux que l'on enmple.

Dans les compes de jardinage, la somche qui porte lempreinte du martean de l'Ẻat doit ètre représentio par ladjudicataire: pour eviter des recherehes tries longues de la part des agents opérateurs, il convient que lemplacement de ces souches soit indiqué par un jalon. Certains cahiers de clanses spéciales imposent aux adjudiealaires lobligation de faire placer ees jalons; dans ce cas, les gardes nauront quä reiller a lexécution de cette clause; 
dans le eas contraime ils doivent faire rux-meme er travat aree laassistance de ladjudicatare on du lacleur.

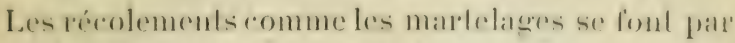
vireses: les gardes, all nombre de trois, quatre on cinf, marchant en ligur, appelent par leur essence de leurs qualitis de baliveaux, modernes on anciens fous les arbres portant lempreinte du martean of le signe correspondant. Chaque arbre crié est immédiatement griffé, et le lien qui l'entoure coupé.

Il est bon de marquer les modernes de deux coups de griffe, les baliveaux et anciens d'un seul, afin que dans les rérifications qui peurent ètre faites, on reconnaisse comment les arbres réservés ont été qualifiés; le geriffage doit toujours être fait du côté de la viréc suivante.

Arant dippreler charque réserve, le garde doit regarder la marque; cest l'empreinte du marteau et non làge d'un arbre qui règle la qualification it lui donner au récolement. Ainsi, dans le balirage. on a pu marquer un ancien comme molerne ef le frapper de deux empreintes, cest comme moderne quil doit ètre appelé; on appellera aussi comme modernes les brins de l'àge, sils ont été marqués de deux empreintes. Les réserves brisées ou abattues par les rents, les accidents d'exploitation, etc., doivent ètre désignées par les mots brisé, chablis, etr. 
Les arbres qui sont réserrés par l'adjudicataire sans ètre marqués du marteau, sont distingués par les mots non marqués.

On doit rérifier arec soin si l'empreinte du marteau existe réellement sur les blanchis. Dans beaucoup de contrées. les buicherons ont lihabitude de ne jamais laisser sur pied un arbre non marqué. sans lui donner ce quils appellent le baptême, c'esta-dire sans le blanchir à la patte: Lorsque la sère de deux ans a recourert ces flaches, il est difficile, a moins dun examen attentif, de les distinguer des véritables blanchis du martelage; aussi arrive-t-il quelquefois quion trouve au récolement beaucoup plus de baliveaux quil n’en a été marqué.

Cet inconvénient ne se produirait pas si, pendant la durée des exploitations, les gardes apposaint lempreinte de leur marteau sur tout arbre non marqué, réservé par ladjudicataire ou ses ourriers. auquel on aurait apposé un blanchis.

La criée doit ètre nette el distincte. Il est plus difficile dans les récolements que dans les martelages d'éviter les criées simultanées, dabord parce que chaque criée niest pas précédée du choc du marleau, et ensuite à cause de la rapidité plus grande de lopération; en se regardant souvent, les gardes sauront prendre thabihule dappeler a leur tour et sans confusion. 
I.es récolements des souches, daus les coupes ell délivrance, deviennent fort pénibles si lon n’a pas pris à l'arance le soin de marquer l'emplacement quoceupait chaque arbre exploité; si au contraire les souches ou les places qu'elles occupaient sont jalonnées, les gardes n’auront qu à ramasser et it compter les jalons en ayant soin d'apposer l'empreinte de leur marteau a côté de celle du marleau de l'État. Cette marque sert à vérifier le complage et à éviter la confusion dans les exploitations subséquentes. 


\section{CHAPITRE XII \\ DÉGATS DES ANIMAUX}

I.E- Trotpeacx, - Betes à cornes. - Elfets du paiturage dansles hois. - Défensabilite. - Bèles a laine. Dérastation des montagnes. - Ilise en défens. - Chèrres. - Pores. - Le grbien. - Le cerf - Le daim. - Le cherreuil. - Le sanglier. - Le lapin. - Lerureuil. - Les petits rongeurs. - Les carisassilms. Le loup. - Le renard. - Battues. - Aplats emporsomnes. - Fouines putois et beleltes. - Chats. LEs orseatx. - Gibier plume : moyen de le conserver. - Oiseaux de proie. - Ltilité des oiseaux nocturnes el des insectirores. - Les risectes. - Les bostriche-

- Lhỵlésine du pin. - Le bombyx du pin. - Le hanneton. - La courtilière.

Les troupeaux. - Les forets serrent de retraite it un grand nombre l'animanx saurages; elles sont en ontre parcomrues par los trompeaux que lhomme? introduit pour quilss'y nomrisient des herbages ef des graines des arbres. La présence de fous ces animanx a. sur la régétation. une influence qüil est nécessaire de ennmaitre afin de saroir distinguer ceax qüil importe décarter ou de détruire de ceux dont il laut, au contraire, lavoriser la multiplication.

Les animaus domestiques. pémis en troupeatu 
mombrous, a liabri de loml dimger, soml plus muici-

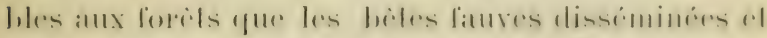

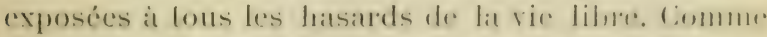

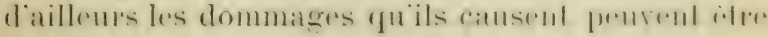

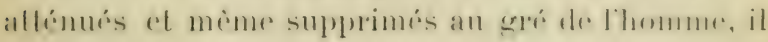

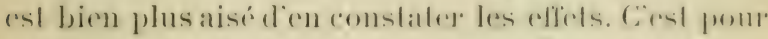
rela quaraml de lrailer des dégals oceastommins pall

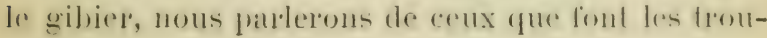
peatux.

Les animamx domestirfues qu'om infroduit or fimaipement dans les bois soml: les bieles a cornes, les

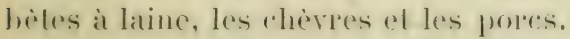

Les biles à cornes, qui se nomrrissent dirrlues of de leuilles, détruisent une grande quantite de jenmes plants, soit en les broutant en mème temps que lherbe, soit en les écrasant sous leurs pierls. Elles mangent les feuilles tendres, les jeunes pousses, et, pour les atteindre, courbent arec leurs cornes les brins dont la cime est hors de la portée de leur bouche.

Le sol des massit's loresliers bien complels étant ordinairement dépourvu d'herbe, le bétail se rejefte dans les cantons mal plantés et dans les clairieres où les herbages sont abondants; la il broute tous les plants qui auraient pu garnir les vides, et il contribue ainsi à maintenir, en l’aggravant, le maurais état de ces cantons. Laspect d'un bois abandonne au par- 
cours differe tellement de celui diun bois en défens qüil est impossible des y tromper.

Le bois interdit aux bètes à cornes est peuplé de brins plus au moins ser'rés, mais formant massif; las clairières, s`il y en a, sont garnies de morts-bois, au milieu desquels on roit surgir des bois blanes et de jeunes sujets dessences précieuses. Les chemins intérieurs sont pelousés, des bordures épaisses entourent les limites extérieures; la régétation envahit tout. Les bois lirrés au parcours sont, au contraire, Inujours parsemés de clairières garnies d'une herbe courte et serrée. Le peuplement se compose de cépées Juissonnantes entremélées d'arlures dont l'écorce est r.'mulue luisante par le frottement réitéré des bètes à cornes. Les chemins piétinés portent des traces nombreuses du passage répété des troupeaux. Ces emfreintes se retrourent dans lintérieur du bois et forment. quand le sol est argileux, des trous profunds ou leau séjourne. Les terrains ainsi pétris sont impropres d la germination des graines. On iroure gi et lit. dans les cépées, des brins ployés et brisés par les bestiaux: partout enfin on roit des traces de destruction sans aucune apparence de reeru.

Le parcours des bètes à cornes a pour résultat dappaurrir le sol. de mettre obstacle au repeuplement uaturel et de multiplier les clairicres ; cest dire assez rombien il est à désirer quion puisse interdire com- 
pletement laceis des forits a ces mimanx. Si les agriculteurs savaient calculer tout ce quils perdent en enroyant leurs troupeaux chercher dans les bois une nourriture insulfisante, ils renonceraient, comme on le fait dans tous les pays bien cultivés, à ce paiturage aussi mauvais pour le bétail que pour la lorì. Mais beaucoup de contrées sont privées de prairies naturelles et les prés artificiels n'y peurent réussir; là, pour entretenir le bétail nécessaire à la culture, on est obligé d utiliser toutes les ressources. Le pâturage des forèts, qui permet d'économiser les fourrages pendant une partie del'année, est done un mal quil faut subir en essayant den atténuer les effets.

Pour cela on devra ne laisser pénétrer les troupeaux que dans les cantons où ils ne peuvent causer de grands dommages. Ces cantons sont ceux qui portent des peuplements bien complets àgés dau moins 10 ans pour les bois feuillus et de 15 ì 20 pour les résineux. Toutes les parties des forèts qui sont clairiérées doivent ètre interdites; on interdira aussi aux troupeaux l'entrée des futaies en cours de régénération et celle des taillis pendant les deux années qui précèdent l'exploitation.

Ces interdictions s'expliquent naturellement par la nécessité de conserver les jeunes plants qui doivent assurer l'avenir de la forèt. 
() uelyues proprictaires se laissent persuader par leurs fermiers yuatpres quatre ou cint ans les bois taillis sont délensables; diantres. plus crédules encore, sont convaincus que le bétail ne mange que lherbe, et ils le laissent pénétrer dans les jennes compes. oil elle est trè abondante. Les bois ainsi liIrés aux troupeaux sont roués a la destruction; il vatudrait autant les délricher tout de suite que de les laisser lentement se transformer en manrais piiturages.

Si le parcours du gros bélail est muisible aux lorits, le pacage des moutons at des chères l'est encore hien davantage? (onoigue des lois tris anciennes aient prohibe lintrouluction de ces animanx dans les bris, il est des contrees ou ils y sont encore admis : a sont les pays monlangenx et pampes, ou loindustrio pastroule peul seule se soutenir. Ce que les montons et les cherres font de mal dans ees contrés est incalculable. Ces animand difruisent les forels non senlement parce quils boutent aree avidite loutes

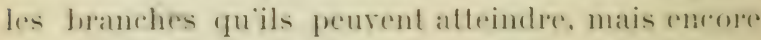

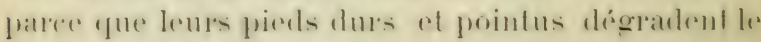
sol ct lonl preir tomles les semences et les jemnes plants.

La presener des heles a laine sur les momlatemes

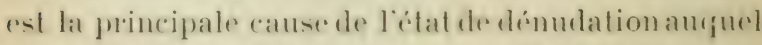
rlles solul arrivers. Les moutous marchant en lrou:- 


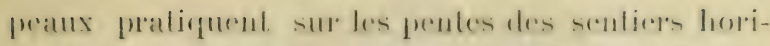

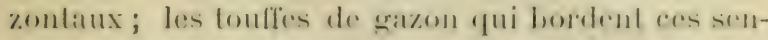

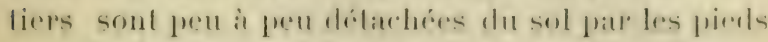
(1. ces animaux el par les cofforts quils font pome en

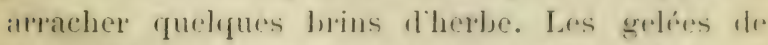

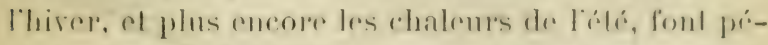

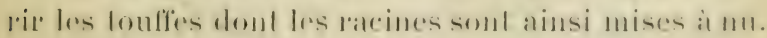
Comme les herbages sont toujours pacagés avant divoir produil leuls graimes, les vides voul salls cesser en satecroissant. Lestrompeatux qui suivent tonjoms lis momes sentiers finissent par en desigregore la

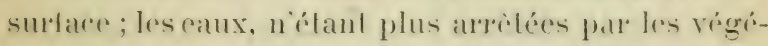
laux, creusent des ravins dout la profondeur augmente à chaque orage; apres quelques amneses, la terre a disparu avee la pelouse. Alors, au liru lu tapis de verdure qui les couvait, les rersants dénudés ne présentent plus qưune surface aride, sillonnce de rarins el completement improduclire.

Quelles que soient les précautions prises pour uliminuer les inconvénients du parage des moutons dans les montagnes boisées, on ne pent jamais empècher 'füil soit tres muisible. Mais on pent exiler qüil deriemne désastreux, en réduisant le nombłe des animanx et la durée du pacage. Ainsi on pour'a fixer, suivant la déclivité et la fertilité du sol, le $n o m b r e$ de moutous admis au pacage a 3 nu 4 tibs au plus par heclare. C'est ì peu pres tout ce rue 
peut nourrir celte surface de terrain à l'état de pàturage boisé. Lorsquion sapercerra que le sol se ravine et que les gazons se dégarnissent par place, on mettra la montagne en défens pendant tout le temps nécessaire, pour reconstituer sa courerture végétale.

Les chèvres sont encore plus nuisibles aux bois que les moutons, parce quelles préfèrent à tout les f'euilles et les jeunes pousses quelles savent atteindre en se dressant sur leurs pieds de derrière. Tout propriétaire désireux de conserver ses forèts en interdira complètement l'entrée à ces animaux. Les pores ne font pas autant de mal aux forêts que les autres animaux domestiques. En fouillant le sol pour y trouver les graines et les racines dont ils se nourrissent, ils déracinent bien quelques petits plants; mais si l'on a la précaution de ne pas les laisser séjourner longtemps à la mème place, ils ne causent pas de grands dommages. Le pore déIruit une grande quantité de larves d'insectes qüil va chercher sous la mousse, il poursuit aussi les mulots et les serpents, et peut, à raison des serrires qu'il rend sous ce rapport, ètre considéré comme un animal plus utile que nuisible. L'introduction des troupeaux de pores dans les bois noecasionuera pas de dommages si on ne les laisse pénétrer que dans les grands taillis ef les perchis, où ils 
peuvent, sans inconvénicul, manger les glands ol. les lainss. car les plants que ces semences produiraient ne pouraient prospérer sous le couverl. II faudra leur interdire l'accès des coupes de laillis deux ans au moins avant lexploitation. ef celui des coupes densemencement des fulaies, afin de conserver toules les graines qui doivent reconstiluer les nouveaux peuplements. Si dailleurs on a soin de ne pas laisser le troupeau sarreter longtemps sur le mème point et fouiller trop profondément, son passage, loin de laisser des traces fàcheuses, produira un bon résultat, parce quil aura pour effet d'ameublir le sol et de le purger d'une grande quantité dinsectes et de petits rongeurs.

Le gibier. - L'influence que les animaux sauvagres exercent sur les forèts quils habitent dépend de leur mode dalimentation. Ceux qui se nourrissent de matières régétales, telles que feuilles, semenees. écorces et racines, sont toujours plus ou moins nuisibles. Les carnassiers devraient, nu contraire, itre regardés comme utiles, parce quiils détruisent les premiers. Mais à còté de lintérèt purement forestier qui commanderait la suppression complète des herbivores et des rongeurs, il y a lintérèt de la chasse qui en exige la conservation. Il faut, pour concilier ces deux intérèts opposés, empècher le gibier de se multiplier assez pour causer des dommages sérieux 
el détruire les carnassiers, qui lui lont une chasse trop active.

Les chasseurs mettent, en général, assez de zèle a sacruilter de leur mission destructive. pour que les forestiers aient rarement à intervenị afin dempicher le gribier de devenir trop abondant. Leur roble 1. plus habituel est de laroriser sa multiplication: dans certains cas, cependant, ils sont obligés de suppléer à linsuffisance des moyens employés jal les chasseurs et de procéder eux-mèmes à la destrurlion danimaux derenus nuisibles par leur nombre.

On distingue, parmi les animaus silurages herbivores, les grands funtes, les betes noires et le pelit gibier. Les grands faures sont les cerfs. les daims et les cherrenils. Les sangliors sont désignés sous le nom dr betes noires a lis lieres of les lapins forment le menu gibier.

- Iecert se nomril des fruil-des lorels, il broute les jemnes jomsises des chenes, des charmes et less fenilles des ponees: il va au gagnage dans les plaines rultivéres, wi il rause de eramels régits.

Vers la fin de lihver. les certs perdent lem bois, qui repousse immédiatement. Ils ont refait leur tète vers le 15 juillet, éporque ou ils commencent a froller leur bois contre les jeunes arbres pour faire lomber la peau qui le recoure. En 


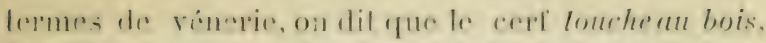

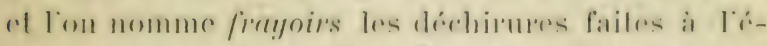
coree des arbers. Les dommages que causend les cerl's ne sont pas tries semsibles dans les laillis el les peuplements naturels hien complete, wit la perte de queleques sujels a pen doimproplanee: mais ils lout un forl tres appréciable aux bois déjat rlairs el surtoul anx planlations darbes verts, paree quils hrisent beaucoup de jeunes arlues en touchant au bois.

Quand les expes deviement assez nombreus poul compromettre l'état du peuplement, on les chasoe con battues. Ces destructions se font aux mois de septrmbre el doctobre. Ciest at ce moment que les animaux sont en renuison. La chair des rerfo tués an fusil, à celle éporgue, est assez bonne, lamris 'qu'elle devient mauvaise si l'on alteml la saison du pul.

- Le daim a des habitudes analogues à celles du cerl, se nourriture est la mème, et au point de rue forestier il ny a aucune différence à faire entre ces animaux. Ils sont égalemont nuisibles sils deviennent trop nombreux. Il est rareque les forestiers aient besoin de recourir a l'aide des tireurs pour arrèter rette trop grande multiplication; mais il nest pas rare, au contraire, de roir les chasseurs demander aux forestiers les moyens de faroriser la reproduction de ces belles espèces. 
Les procédés de conservation les plus simples consistent d'abord à détruire autant que possible les loups et les renards, qui font une chasse acharnée aux jeunes faons; puis à sabstenir de tuer les biches. Il faut ensuite réserver quelques ronciers, qui les abritent et leur fournissent du feuillage rert pendant lhiver ; puis, quand la terre est couverte de neige, déposer dans les endroits fréquentés par les hardes de cerfs des fourrages. des faines et des pains fabriqués avec de la terre glaise et du sel.

Gràce à ces précautions, la reproduction des grands fauves sera assurée et l'on n'aura pas à craindre de les voir sortir du bois pour aller au gagnage dans la plaine, où ils sont exposés à ètre tués par les bratconniers.

- Le eherrenil est moins nuisible aux foréts que le cerf et le daim ; il fait aussi moins de dégàts dans les champs, parce quil quitte rarement le couvertoù il trouve des jeunes pousses. des faineset des feuilles de ronces, qu il préfère a toute autre nourrture. Au printemps, il mange les jeunes pousses de bourdaine; cette nourriture produit chez lui une espèce dirresse qui lui fait perdre le sentiment du danger. 11 n'est pas rare de voir, a celte époque, des cherreuils affolés pénétrer jusquau milieu des villages ct approcher de lhomme, quils fuient en fout autre lemps. 
Le cherrenil mate prend le nom de brocard: la liemelle, celui de chevrette. Le brocard a, conme le cerlí la tèle ornée de bois qui tombent tous les ann rers le mois de novembre. Sa lide est refaite rers la fin de janvier.

On emploie, pour atsinere la multiplication de res animaux, qui sont fort recherehés des chasseurs a cause de la délicatesse de leur chair, les moyens indiqués plus haut pour le cerf el le daim. C"est en détruisant les loups et les renards, en respectant les cherrettes, et en réservant des ronciers, qu'on arrive le plus surement at conserver les cherreuils dans les forèts où il y en a déjà.

Il est beaucoupplus difficile de les introduire dans celles d'où ils ont disparu, car les animaux adultes quon y transporte, se trourant dépaysés, n’ont pas de refuites, et ront sourent se faire tuer dans la plaine. Il est préférable d'élever en demi-domesticité des faons, quion rend à la liberté au moment du rut. Les cherrettes ront mettre bas dans les fourrés, et si l'on peut préserver les portées des atteintes des braconniers et des carnassiers, on aura de nouvelles générations habituées à la vie sauvage.

- Le sanglier se nourrit, comme le porc, de fruits, de glands et de racines. Il sort des bois pendant la nuit et va dans les champs de blé, d’avoine, de maïs el de pommes de terre, ou il lait de grands ravages. 
Il dérore ausi les lerrauts et les lapereaux yuiil trouve dans leurs rabouillères, les faons de chrereruils et les poules faisanes quil saisit sur leurs wuls. C"est un aninal éminemment destructeur. que les lorestier's ansi bien que les chasseurs ont interèt à poursuivre à outrance.

Le's sanglier's ne sont point sédentaires: pendant quelques amnées, on les tronve en grande abondance dans certaines forets : puis on les roit tout din coup se portei dans les lorits tres éloignées, quils quitteront de mème sans quion puisse se rendre compte des causes de ces déplacements.

On dome le nom de murcussin an jeune sanglier. tant qu il porte la lirrée, rest-it-dire tant que son felage est mardue de bandes faures et noires. Plus tard il devient bè de compurgnie, raygl. liers-an. puis solituire. lorstue, derenu plus itge, il cesse de laire partie d'une bande.

fon détruit les sangriers au moyen de battues, et plus sùrement encole en entomant de tireurs lis

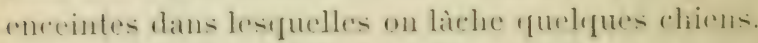
Les sangliers, harecles poll ces adrersates plusbroyants que pedoutables, se lassent de leurtenir téln, et finissent par se décider a vider lenceinte. $\ddot{C}_{i=1}$ ce moment que les ehasisens saisissent pour les tirer au passage.

Il est inutile de sacharner at poursuire un ragot 


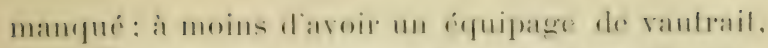

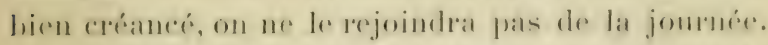

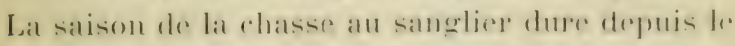

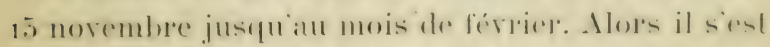

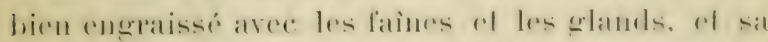

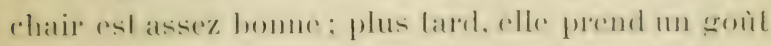

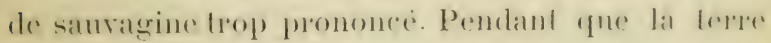

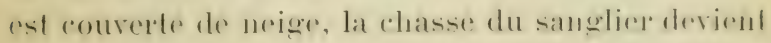

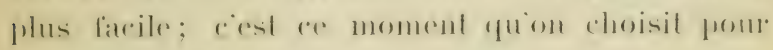
opérer les grandes destructions.

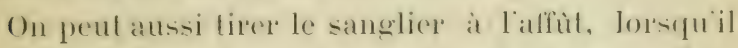
1a, dans larriere-saison, retomrner les clumpes di" pommes de lerre et de maiss ; mais, comme cet animal a lodoral tres fin, il exente somrent de lost loin l. rhassemr, qui se morfond en latlemdanl.

Arec une bide aussi muisible il $11 \mathrm{y}$ a ancun monatrement à prendre; il laut sen débarrasser par lous Jes moyens. Les précautions quion pourrait premdre pour réserver les porties seraient presque toujours sans profit, car l'humeur de ces animanx est trop vagabonde pour que le chassiur puisse esperer lefrouver en allomme les natreassins remeontres all printemps.

- Le lieve et le lapin, quoiqu. tris raplyochís jar leur conformation, difierent sensiblement yar leurs mceurs. On troure le lière dans les plaines cultivées, les bruygeres, les buissons et les lisieres 
des bois. Le lapin plus exclusirement lorestier, ne quitte guère les bois et garennes; il creuse des terrier's profonds dans lesquelsil se réfugie, tandis que 10 liève vit toujours à découvert el n’a, pour se préserrer de ses nombreux ennemis, que la ritesse de ses jambes.

Les lapins, comme les lièrres, se nourrissent dher Jues, de céréales, de légumes, de bois tendre et d'écorces.

Les lieves deriennent rarement assez nombreux pour causer des dommages sérieux aux forèts. (“est seulement dans les parcs quils se multiplient de manière a devenir nuisibles; mais il est toujours lacile den réduire le nombre. Il n'en est pas de mème du lapin. Ce rongeur est un réritable fléau pour les bois, et les forestiers ne sauraient prendre. trop de soin pour le détruire.

Les lapins font les plus grands dégails en hivel : quand la neige courre la terre, ils rongent lécorce des jeumes brins de charmes, de trembles, de chènes ct broutent les feuilles et les bourgeons terminaus des plants d'essences résineuses.

Il est impossible de faire des repeuplements et de conserver des pépinières dans une forèt infestée par ces petits animaux, qui se multiplient arec une effrayante facilité; car la femelle peut faire cinq ou six portées par an. 
On dedruil les lapins an moyen de ballues somvent répélées. On emploie aussi les furets, qui les font sortir des terriers où ils se réfugient; beancoup de propriétaires donnent à leurs gardes la permission le chasser le lapin : e'est une lrès bomne mesure, it rondition que les gardes ne les ménageront pas pour se réserver une ressoure alimentaire qui est fort prisée par eux. Quand on veut sérieusement purger un bois de ces hotes dangereux, il faut prendre. le parti décisil de les considérer, non plus comme un gibier quil est amusant de tirer, mais comme un animal nuisible dont il faut se débarrasser à tout prix. Pour arriver à ce résultat, quelques propricilaires donnent à tous les chasseurs du pays l'autorisation de tirer le lapin dans leurs bois, ils permetlent dy faire des battues, de fureter, et parvieunent ainsi, sinon à détruire entièrement ces animaux, du moins à areter leur trop grande multiplication.

On peut atténuer les dégâts que les lapins causent cn hiver aux jeunes peuplements, en plaçant autour de leurs terriers des brindilles coupées de charme. de tremble, sur lesquelles ils exercent leur actrité; mais ce sont là de faibles palliatifs, et le plus sûr est encore d’appliquer les procédés destructifs.

- Nous ne mentionnerons, parmi les autres rongeurs, habitants des lorèts, que l'écureuil et le mulot. Le premier de ces animanx lait quelque tort aux 
arjeres résineus, parce qu il hrise les pousses termimales et ronge les ivailles des cones pour se nourrir des sraines qüils renlerment: mais dans les bois firuillus il est à peu pris inoffensil. On le considere copendant comme muisible, parce qu il pille les nicls: mais. comme il mesittafue gutre qüa cemx des pies ef des geais. on pent lui pardommer, de rason du pe:u dintriet guoline la conservation de ces oiseaux. beaucoup plus destructeurs que lui.

Le mulot latit de grands diegits dans les pépiniepes (i) les semis artiliciels. C̈est un animal éminemment nuisible, dont il est diflicile d'arèter la multiplication; on le détruit aves des pieges ordinaires et des pots de terre rernissis à linterieur, yuion enderme dans le sol. en les remplissant dean a moitié. Les mulots sy laisient tomber el se noient apres avoir latil des (rforts impuis-ants pour grimper sur les parois lisses.

L. mogen le plus simple et le plus ecomomiegue

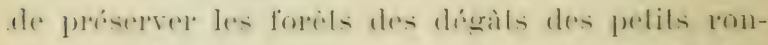

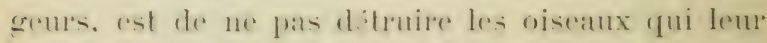

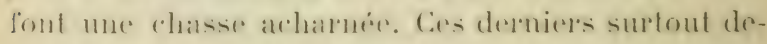

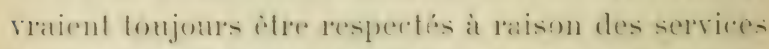
signalis qüils remelent. Lön senl dentre cux, remarfualile flar sa taille el assez rave, le grand-eluc, rhasse le lierre et le gribier ; tous les autres so nomelisicut furesfue exelusirement de souris, de mulots 


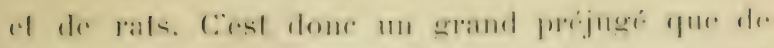

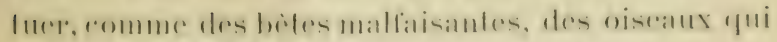

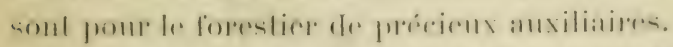

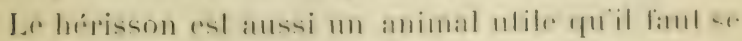

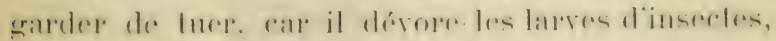

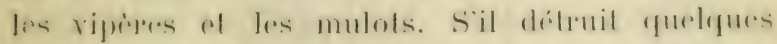

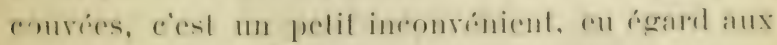
services qu'il rend d'autre part.

Les carnassiers. - Ies rammasicies los plus lorpandus dans les foreds soml les lomps. les ponatrolse les rlats samvages, les fomines, les beletles of les jufris. Tous ees animanx sonl les emmemis les plus dangereux du gibicer; anssi sont-ils regardés par les chasseurs eomme deseoncurrents redoutables qüils poursuivent à outrance.

Le loup et le renart se chassent it comre ef en battues. Nous ne parlerons pas du premier procédi. fui est du domaine de la vénerie; mais nous indiquerous quelefues précautions nécessaires pour rendre les battues fructueuses.

La premiore de toules est de crarder le phus romplet silence quand on plaes les tirems et les rabatleurs. Ciest compromettre bénérolement le suceis diune chasse que dirrixer à grand fracas prés diune enceinte oì lion a détommé lanimal. Il est probable quil a depuis longtemps flairé le danger et pris les grands derants. 
Dans reptains pays, la population entière se met ell mourement pour une battue aux loups. Les rabatteurs se lont suivre de tambours, ils portent des crécelles et crient à tue-tète pendant qu ils sont sous bois. Il est oiseux dajouter quion ne tue jamais rien dans ces bruyantes expéditions. Quelques chasseurs placés à bon rent, à portée des roluites, valent mieux quine bande nombreuse de tireurs inexpérimentés. Si les rabatteurs sont guiles par des gardes connaissant bien le terrain, suils marchent saus bruit, rapprochés autant que possible, les loups, aussi bien que les renards et les sangliers, s'empresseront de se dérober devant eux et iront se faire fusiller sur la ligne des tireurs. si, au contraire, les traqueurs mènent grand train. les animaux effarés partent dans toutes les directions c. forcent souvent la ligne des rabatteurs.

On se débarrasse aussi des loups et des renards arec des appâts empoisonnés. Le poison le plus sùr ist la strychnine, quon extrait de la noix romique. Il suffit diune pincée de cette substance pour faire pírir instantanément un grand loup et à plus forte laison un renard. Quel que soit le poison employé, slrychnine, noix romiqne, arsenic, il faut bien se garder de le metlre comme on le fait quelquefois. dans des bètes mortes laissées au milieu du bois. On risque daroir empoisomme tous les ehiens 
du pays avant yuin senl loup se hasarde it mordere it lappat. Pour éviter le danger qu'offe lemploi du poison, on devra ne s'en servir qu'à l'époque où, la chasse étant close, les chiens ne fréquenteul plus les bois. Le meilleur moment est le mois de mai, cyougur oì les animaux carnassiers, ayant despetits it nomrrir sont obligés de chasser très actirement. On se procurera des mulots, des souris, des grenouilles ou de: petits oiseaux récemment tués. On introduira dans le corps de ces animanx une pincée de strychnine, d'arsenic ou de noix vomique, et on les placera dans les plaines qui entourent les forèts, et non dans les forèts mèmes. Les loups et les renards qui font totites les nuits leur randonnée antour des bois. pour prentdre les levrauts, les oisillons et les mulots, troureront les appàts et les dévoreront sans méfiance. si l'on s'esl servi de strychnine, ils resteront sur place; ils iront, au contraire, mourir dans quelque buisson éloigné, si l'on a employé la noix romique ou l'arsenic, parce que l'action de ces poisons est bien moins prompte.

Les fouines, putois et belettes sont d'autant plns dangereux que leur petite taille les rend plus difficiles à atteindre. Ces animaux se nourrissent exclusivement de proie virante et d'œufs. Ils sont redoutables pour les faisanderies, les parcs et les bassescours, dans lesquels ils font de grands ravages.

On les prend aree des pieges dits assommoirs on 


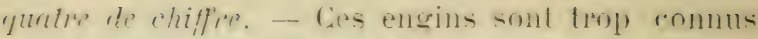
jenur quil soit népesaire de les decrire.

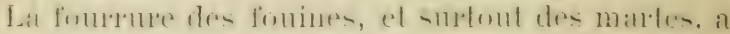

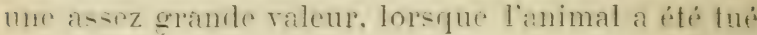

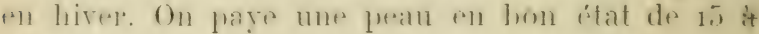

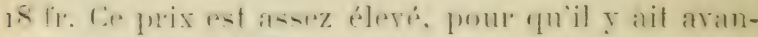

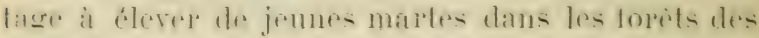
montagnes. oir, daillents. le froid et les neiges rendent impossible la eonserration du gibier.

Le that samage et te ehat domestique. Inesqu'il a mis lihabitude de courir les bois, sont de grants

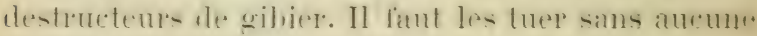
miséricorde.

Les oiseaux. - Les niseaux qui habilent les foriti

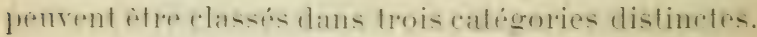
Cenx qui sont considfrés romme gihier. savoir : les

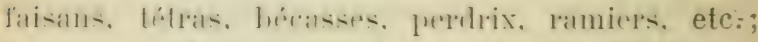
les oiseanx de proie, diurnes of nocturnes : aigles. faucons, buses, chats-huants, etc., et enfin les granivores et insectivores.

Le cribier phume ne fait antemn fort an bois. Le

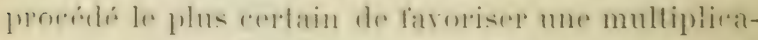
fion qui ne gresente aueun ineonvénient consiste

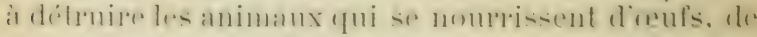
petitem doiseaux adulles. Tous arons déja signall: romme les ememis les plus dangerenx du gibien pwil. les loupr. les renards. les sangliers, les fonines 


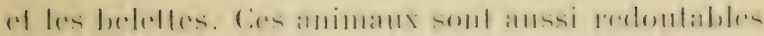

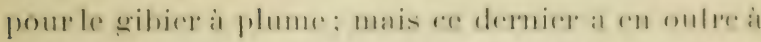

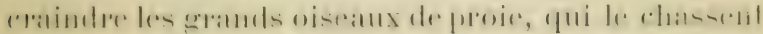

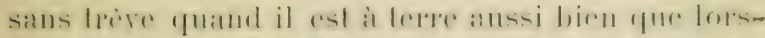

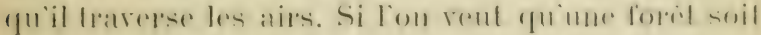

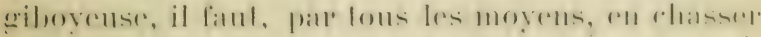

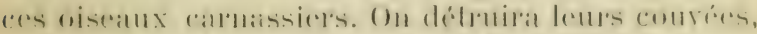
on temdra des piegess sur la reme des anbers oil ils ont lhabilude de se postere. On les lirema rhatque fois quil se presentera me oecasion favopalule.

Nous arons dit précélemment rua l'exception du grand-due, les oiseams de mit : ehometles, hiboux, chats-huants, sont de gramds destructeurs de mulots; ils font en outre une finorme consommation de gros insectes, tols qur hametons, phatines, sphinx, fort muisibles aux arbores, of méritent, it rilison de ces services, me protection toute spéciale.

Il lant que les forestiers salfranchissent entierement du préjugé absurde qui voue à lat mort eos oiseanx de nuit, qui nont jamais lat que du bien. En . Dllemgne, la chouetle, qui pareourt sins bruit l's forèts pendant la muil, en les purgeant de ses ennemis les plus dargereux, est considérée comme lemblime du forestier vigilant ef respectée de tous. A lexception du grand corbeau et de la pie, quon accuse de détruire les nids, la plupart des oiseaux forestier's sont insectirores et sort, par cela 
mème, de précieux auxiliaires du sỵlviculteur. Tous les becs-fins, les mésanges, se nourrissent des larves et des insectes qu ils récoltent sur les arbres; les loriots, les coucous, dérorent les grosses chenilles relues; les pics eux-mèmes, auxquels on reproche de percer des trous dans les trones diarbres, ne font pas de dommages sérieux, ear ils ne s'attaquent qu à des arbres déjà enrahis par les insectes.

En général, on peut dire que la présence des oiseaux dans les bois est éminemment farorable: le!; forestiers doivent donc faire tous leurs efforts pour empècher la recherche des nids, la chasse aux gluaux, a la raquette et tous les moyens de destrurtionsi fort en honneur dans les campagnes.

Ils derront surtout sattacher à expulser les gamins qui vonl, au printemps, courir les bois dans le seul but de fouiller les nids. Il est difficile de faire comprendre à res dévastateurs inconscients tout le mal quils font; mais quelques coups de gaule bien appliqués suffiront pour leur faire mir 'qu'il n'est pas permis d'aller récolter sur les arbres les matériaux d'une omelette.

La conservation des petits oiseaux est aujourdhui considérée comme d'intérèt général ; il est très important que les gardes des forèts soient les premiers it donner lexemple de lobéissance aux règlements qui protigent ces utiles destructeurs dinsectes. 
Les insectes. - I), lous les dres viranls qui sallapuent aux forels, les insectes, les plus petils de lous, sont les plus nuisibles. Leme nombre leur voracilé les rendent dangereux, non seulemenl pour quelques arbres isolés, mais pour des peuplrments entiers, dont ils penvent amener la ruime.

Le plus redoutable des insectes destrurteurs dro bois est le bostriche. Ciest m petit coleoptere, long de 3 à $\{$ millimetres. Aux mois d'avril et de mai, les bostriches voltigent autour des épicéas dépérissants ou récemment abattus, ils percent l'écorce jusqu'au liber, creusent de petites galeries où la femelle dépose ses cul's, au nombre de 20 à 6o. L'éclosion a lieu quinze jour's après la ponte. Les larves

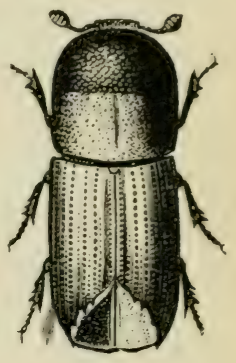

Fig. 16. rreusent a leur tour des galeries latérales qui rout en s'élargissant, elles se transforment en chrysalides à lextrémité de la galerie, puis elles deviennent insectes parlaits, percent l'écorce et sortent pour saccoupler à lextérieur. La durée de ces transformations est de deux à trois mois, suivant la température.

Le bostriche typographe (fig. 46 ) sattaque excluFig 46. - Bostriche typographe (giossi). 
sirement aux foréts dépicéas, dans lesquelles il cause de grands ravages. Il est fort difficile de detruire un insecte qui vit sous lécoree et qui est, far suite, peu exposé aux attaques des autres animaux. Les seuls moyens quion puisse employel annsistent a ne laisser dans les forets ancun bois hiprisisant, a enlerer le plus promptement possible les chablis, les brois abattus, a faire abattre les arleres altargue et it laire brùler les écorces.

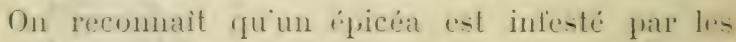
Ju-trieles-anx signes suivants: les aiguilles devienm.nt jaunes et tombent, lécoree prend une coulenr rougeàtre et se détache facilement. elle est parsmen de petits trous dou sechaple une proussine fine, qui est te la rermoulure. On roit, sous lircorce, des sillons nombreux. noirs, pleins diouls et d. larve dinsertes. Lardore presente des cararliares matrqués de dépérisoment, il finit par perelese

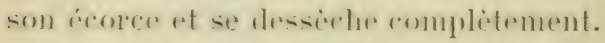

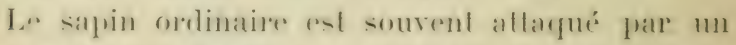
bo-lriche qui differe peu de celui de lépiecat mais

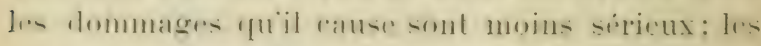
moyeu- proservatifs sont daillemes les memes.

On frout dire, dume maniere sénerale, que ces insertes or multiplient de preference dans les peuplements maladil's et affaiblis. Ciest dans les buis dont le sol ast see. maigre, anx expositions 


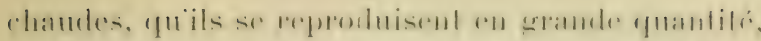

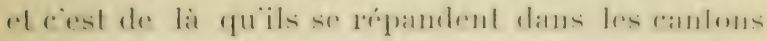

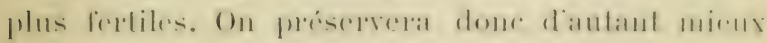

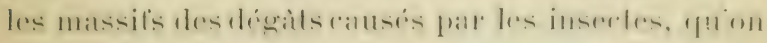

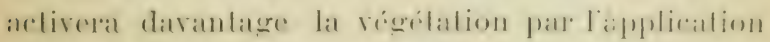

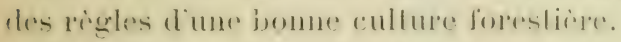

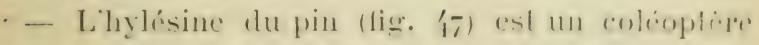
comme le bostriche. Il diffère de ce dernier par la forme de son corselet, qui est plus étroit. La lemelle dépose ses out's dans lécorce des arbres dépérissants ou récemment abattus. Les larres, qui éclosent peù de temps après la ponte, creusent leur galerie dans le liber et y opèrent leur métamorphose. Linsecte parlait, qui sort au mois de juil-

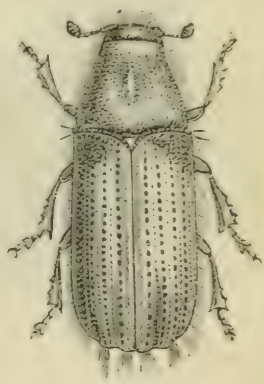

Fir. let, attayue la base des jemes pousses du pin, les

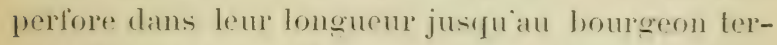

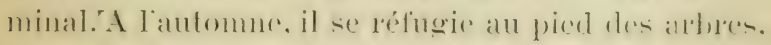
dans la mousse et sous l'écorce.

On reconnait la pésenee des liglesines a lasperel d.s jeunes pousses. qui jaunissent et tombent. Cet insecte lait beaucoup de mal aux pineraies. Cist surtont àux bois dont la régéstation est languissante

Fig. 47. - IIylésine du pin (grossi). 
qu il s'atlayue; aussi, pour s'en préserver, $11 \mathrm{y}$ a-t-il dautre moyen que de débarrasser les peuplements de tous les bois morts ou dépérissants.

- Le bombyx du pin (fig. 48) est un papillon de nuit, dont la chenille, qui se nourrit des feuilles du pin sylrestre, est des plus nuisibles; sa puissance de reproduction est si grande et sa roracité si redou-

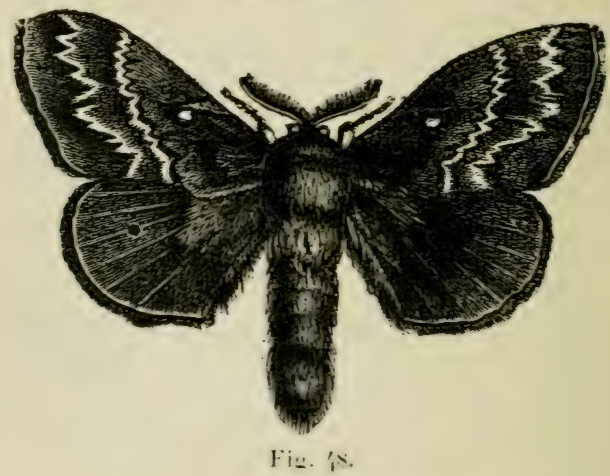

libble. que des lomits entieres sont completement dépouillées de leurs feuille - par ses innombrable's bataillons.

On a en rain essayé, pour les détruire, tous les moyens imaginables. On a tenté de couper par des fossés les cantons attaqués; mais les chenilles sont quelquefois en si grand nombre quelles remplissent

lis. 48. - Bombyx du pin. 
les fosses ef finissent par les franchir. Le send procide praticable est de recueillir el de brùler en liver la mousse dans laquelle elles se cachent.

- Le hameton est un coléoptere dont la larve porte le nom de man ou ver blane. A liblat parlait. le hammeton se nourrit des feuilles du chine, du hètre, du charme et de beaucoup d'autres arbres feuillus ; il ne s’attaque pas à celles des résineux. Les dommages qu'il cause, quoique très apparents, ne compromettent pas l'existence des arbres forestiers, mais ils ont pour effet de ralentir leur croissance et de détruire les organes de fructification de l'année. La larve ou ver

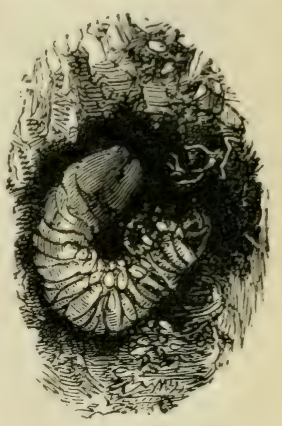

Fi⿳. ㄴ.

blanc produit des effets bien plus lunestes. Ciell. larve (fig. 49) provient dicul's que le hammeton dépose dans le sol vers le mois de juin. Elle éclòt un mois ou six semaines après la ponte et commence à creuser les galeries souterraines dans lesquelles elle vit jusqu'à l'été de la troisième année. A ce moment, elle se rapproche de la surface, se transforme et sort à l'état de hanneton.

Le ver blanc se nourrit des racines tendres de Fig. 49. - Larre du hameton. 
presifue tous les régélaux: il est redouté des cultivateurs. dont il détruit les récoltes. Sa voracité est tris gramule. Les pépinières et les semis sont très exponis aux rarages de re dangereux insecte. quil

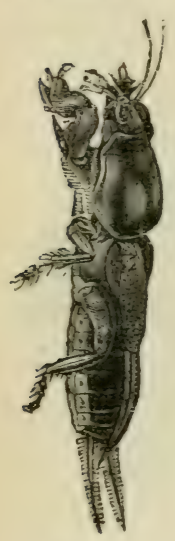

Fi... 站. est trés difficile de détruire à cause de sa vie souterraine.

De tous les moyens employés pour se débarrasser du ver blanc, le seul qui ait domné des résultats consiste a recueillir les hamnetons quand ils sont engourdis dans les feuilles. On secoue énergiquement les jeunes arbres sur les lisièresdes bois, et l'on ramasse les insectes tombes. On peut ainsi en détruire, en peu de jours, dénormes quantités.

Le hannelon difrose plus volonticrs ses ceufs dans la terre ameublie que dans le sol compart des forcits, aussi les cultures qui avoisinent les bois sontelles plus exposées que les atulpes aux devastations de ens larres. Lers

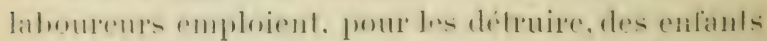
qui les ramasosnt quand la charrue les decouvere. On habitue ansid a faire cette chasse les oiseaux de bascic-cour et meme des chiens, qui sont tres friands de ces larres.

Fig. 50, - La courtilière à l'état d'insecte parfait. 
- La courlilibre (lig. 5o) est un inserle du germe des orthopiers. Elle vil soms lererel ereuse, comme l. vor blane, des galeries dans lesquelles elle fail sa ponte; elle ravage les pépinieres, les jandins, en devorant les racines de tous les végétaux quielle. reneontre dans le traté de ses galeries.

La courtilise est pourve dailes dont rlle se sert pour quitler, vers le soir, sa demenpe souteraine. La femelle pond justgu à 200 cenls, qui éclosent au brout d'une ou de deux semaines. Les larves sont tres rorares el se jettent arec avidité sur toutes les matieres régétales; aussi une seule courée suffit-elle pour déraster entièrement une plate-bande de pépinière.

On détruit cet insecte en recherchant, pendant le mois de juin, les nids dans lesquels il dépose ses wufs. Ce nid nest pas à une grande profondeur: un coup de bèche sulfit pour le décourrir. On brise la corque de terre durcie au milieu de laquelle les arul's sont placés, ot ceux-ci périssent au contact de l'air extérieur.

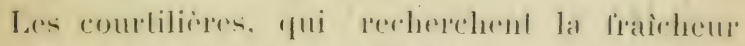
pendant les sécheresses de lede, roul souvent se rélugier sous les planches et les paillassons mis a plat sur le sol. On peut détruire un grand nombre de ces insectes en disposant, dans un coin de la pépinière, quelques vieux paillassons, quion relèvera au milieu du jour. 
En signalant dans ce chapitre quelques insectes des plus connus et des plus redoutables, nous n'arons eu d'autre but que de donner une idée succincte des ravages qu'ils causent et des moyens yu on peut employer pour les prévenir. Nous renvoyons ceux de nos lecteurs qui voudraient étudier plus complètement cette branche des sciences forestières à l'excellent petit traité publié par M. de La Blanchère, sous le titre de les Ravageurs des forêts (1).

Lobservation attentive des mours des insectes a lait décourrir quelques procédés de destruction qui peuvent ètre employés arec succès, mais on est obligé de reconnaître que ces moyens sont tout à fait insuffisants en présence des grandes invasions. Lorsque la ponte et léclosion se sont faites dans des conditions favorables, le nombre des insectes est si considérable quion ne peut songer à les détruire. Dans ces circonstances, il n’y a qu à laisser agir la nature, elle se chargera de susciter contre les espèces dont la multiplication est exagérée des ennemis qui auront bientôt rétabli l'équilibre.

Le sylviculteur ne doit cependant pas rester inactif; mais au lieu de poursuirre des ennemis insaisissables à cause de leur petitesse, il cherchera d'abord

(1) Les Rutageurs des forets, par La Blanchere; in-16. an pages, 44 grarures; libr. J. Rothschilı, prix: 2 fr. 
ì utiliser sans retard les arbres gravement compromis, puis il s'efforcera d'activer la végétation des massifs attaqués, de manière à réparer, sinon pour le présent, du moins pour l'avenir, les dommages qu'ils ont subis. Les moyens a employer pour arriver à ce résultat varient suivant la nature du terrain et l'état du peuplement; mais on peut indiquer dune manière générale : le mélange des feuillus avec les résineux, la conservation du sousbois qui maintient la fraîcheur, les nettoiements et l'enlèvement des bois morts, comme les procédés les plus sûrs de prévenir et de réparer les dégâts qu'occasionrent les insectes les plus redoutables. 


\section{CHAPITRE XII}

\section{PISCICULTURE}

Le service de la pèche. - Causes de destruction des poissons. - Éporques de la fraie. - Frayeres naturelles. Frayères artificielles. - Fécondation artificielle. Choix du reproducteur. - Maturité des wufs, de la laitánce:- Manière dopérer. - Appareils d’incubation. - L'éerevisse.

Le service de la pêche. - Le décret du ; norembre 1896, qui a transléré au ministère de lagriculture les attributions en matière de pìche. qu un décret du 29 arril 1862 arait confiées au serrice des ponts el chanssées, a créé pour le persomnel de ladministration des eaux ot forèts de nouvaux deroirs.

Ce nesl pas seulement aux préposés de celle administration qu incombe le soin de supveiller la perche el de faroriser la multiplication du poisson, les gardes particuliers ont aussi les mèmes deroir's. C'est pour donner a ces deux catégories de préposés le moyen de les remplir que nous arons consacré, dans le 2 volume de cet ouvrage, un chapitre aux 


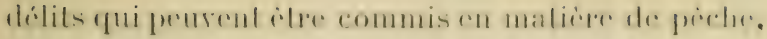

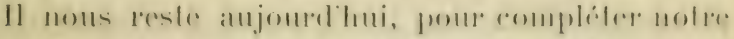

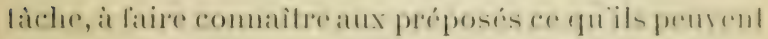

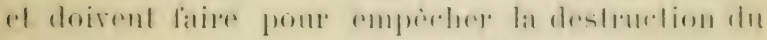
poisson ol litroriser sil mulliplication.

Causes de destruction des poissons. - I in linemulili: des poissons est si grande que leme nomble siarreoilrail jusquall moment oil les ressomeres ali-

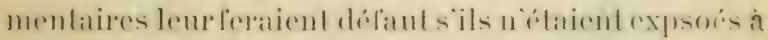
lant de callses de destruction (1). Ainsi lérelosion des crufs est sourent arreté par des changements dans la température ou la limpidité des maux daus lesquelles ils sont déposés. Cés aeul's sont avidement recherehés par les insectes, les oiseaux arguatiques ef les poissons qui sen nourrissent. Les alevins sont à peine éclos quils sont poursuivis pall les rats deau, les canards, les tritons, les dytiques (fig. 51) et les autres insectes aquatiques. Adultes, les poissons sont rictimes des loutres, des chals, des oiseaux de proie et surtout de lhomme qui c'st le destructeur par excellence.

Si, par une surveillance assicluc, on peut arriver it réduire la destruction causér par l'homme, on sanvera bien quelques reproducteurs, mais ce quil lant surtout, cest saurer leur progéniture. La première

(1) La carpe et la tanche portent en moyenne Gu ocio ceufs. la perche et le brochet 50.000 , la truite 6.000 . 
mestre à prendre pour cela consiste à interdire la pèche pendant le temps de la fraie, époque où le poisson vient en nombre sur les points où il doit déposer ses oufs.

Epoques de la fraie. - Qunigure le temps de la fraie

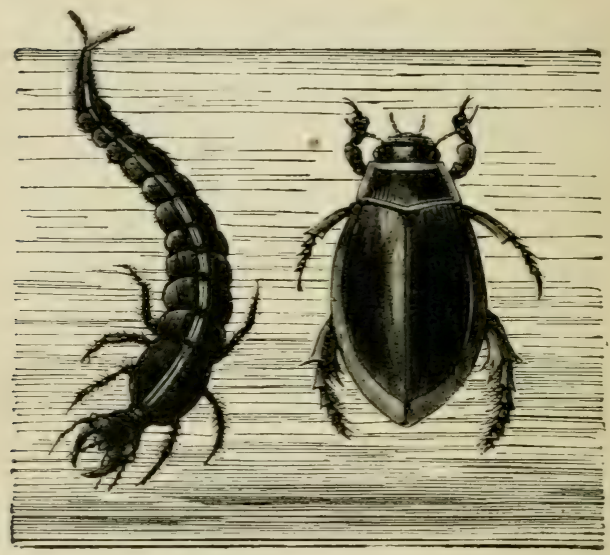

Fin. is.

varie, pour chaque espèce, suivant le climat et la fompriature locale. on peut le tixer diune manière. générale :

Doctobre a janrier, pour le sammon, la truite ot lombre chevalier.

De février it mars, pour le brochet. 
Darril a juin, pour le barbeau, la brème, la perelue et l'ombre.

De juin a fin d'août, pour la carpe, la tanche, le goujon.

Quand les poissons se réunissent en nombre, sur les points déterminés, quils y séjomrnent ef s'y poursuivent, aux époques connues comme propires i la fraie, cest quils trourent la les conditions qui constituent les frayères naturelles.

Frayères naturelles. - Ces frayères doivent elre surveillées avec grand soin, car c'est là que les pècheurs sont attirés par la grande quantité de poissons qu’ils y trourent réunis.

Pour rendre sur ces points la pèche difficile, les gardes y placeront des quartiers de roches, des pieux garnis de clous qui rendent inpraticable l'emploi du filet.

Ils tiendront soigneusement à l'écart des frayères les oies et les canards. Lorsqu'on aura à exécuter des traraux de curage, de faucardage et autres qui ont pour effet de troubler l'eau et d'effrayer le poisson, il conviendra d'ajourner les travaux jusqu au moment où les alevins seront en état de fuir les eaux contaminées. Ce temps est de cinq à six semaines pour les poissons qui fraient l'hiver et d'une ou deux semaines pour les autres.

Si les fraỳères naturelles ont été détruites, par des 
Larauxde dragage, des constructions de digues, cte. il est nécessaire d’en créer d'artificielles. Il faut pour cela connaitre les maurs des poissons auxruels elle's sont destinées.

On a observé que les femelles des poissons dhiver; saumons, truites, au moment de la fraie, froltent leur rentre sur les lits de gravierset y tracent des sillons dans lesquels elles déposent leurs apuls quie les màles fécondent en y projetant leur laitance, ot qüils recourrent ensuite de graviers. Cost dans les caux rires ef froides que sopere cefte fécondation.

Pour créer des frayères artificielles destinées aux poissons ile cette espèce, on choisira, dans un cours dean vive, un point où le courant ne soit pas frès fort ef dont le fond ne soit ni vaseux ni couvert dherbes, on y étendral une conche de petits cailloux roulés et de gravier de 10 a 20 centimetres dépaissem. C̈est la que riendront frayer les sattmons, les truites ed les omtres cheraliers, si dailleurs ils y trourent la tranquillité dont ils ont besoin.

Pour les poissons qui liayent en été el qui allatchent leurs anfs aux herbes el aux racines, on formera avec des lattes des especes de clayomnages (fig. 52) composés de jones, de bruyeres, de menus bois que lon placera it de petites profondeurs sur des plages en pente douce, dans des eaux tranruilles et bien exposées au soleil. 


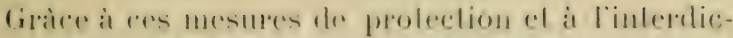
tion des modes de peide prohibris, les cours diean

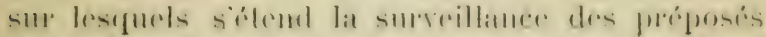
se repeupleront promplement à moins que la destruction y ait éte tellement complete qu il ny reste plus un nombre suffisant de reproducteurs.

Dans ce cats, il est nocessatere de recombrir it la reproduction artificielle qui constilue la pisciculture propremert dite.

Les poissons qui fraient en hiver : saumons, truites, ombres chevalier's, aiment les eaux vives el fraîches, les ruisseaux des montagnes. Ceux qui fraient en été préfèrent les cours d'eau tranquilles, les eaux tièdes, lẹs étangs. Il faut donc approprier
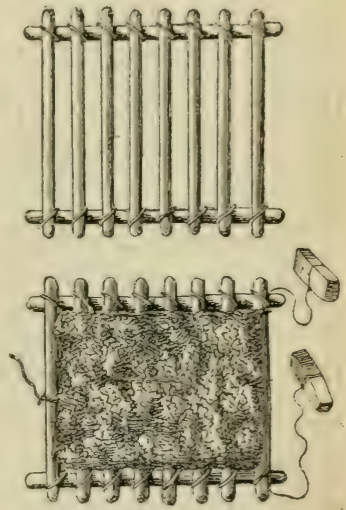

Fig. 52. chaque espèce aux cours d'eau qui hui conviement. ...Les poissons d'été : carpes, tanches, brochets, perches, dont les ceufs adhérents sont fixés aux herbes et aux racines peurent ètre multipliés assez facilement sans recourir à la lécondation artificielle. Il suffit de mettre dans des réservoirs alimentés par des eaux pures et naturellement tièdes, quelques 
couples de poissons adultes de l'espèce qu'on reut propager, pour obtenir des éclosions abondantes, pourru quion ait soin de créer dans ces bassins des frayères artificielles s'il n'y en a pas de naturelles.

Les jeunes de ces espèces se dispersent aussitòt qư̈ils sont éclos et ils échappent, par leur petitesse et leur agilité, à toute protection.

Il nest pas aussi facile de multiplier les poissons dhirer:saumons, truites, ombres cheraliers ; cest pour ces espèces, les plus recherchées pour la qualité de leur chair.qu il faut employer la fécondation artificielle.

Fécondation artificielle. - Le succès dépend du choix des reproducteurs, de l'état des œufs et de la laitance. Les reproducteurs doivent ètre bien vivants. Les ceufs qui remplissent le ventre des femelles sont mùrs lorsqu'ils se déplacent facilement sous une simple pression des doigts et qu ils s'écháppent naturellement lorsquion suspend le poisson dans la position verticale. Au moment de leur chute, les ceufs sains, qui ressemblent à de petites groseilles, sont presque transparents, l'enduit visqueux qui les entoure ne blanchit pas au contact de l'eau. Ceux qui sont opaques et dont la mucosité trouble l'ean sont altérés et doivent être rejetés.

La laitance est mùre quand elle s'écoule sous une légère pression pratiquée sur les flanes du poisson. 
Saine elle a la conleur et la consislance de la creme. Pour procéder a la fécondation, l'operateur se plare rntre deux baquets pleins deau, dont l'un contient le male et lautre la femelle dont il doit se servir.

Il a devant lui un vase a fond large el plat. "n laïence ou en verre, bien propre et rempli drau pure a la tempéralure de 5 à 10 degrés.

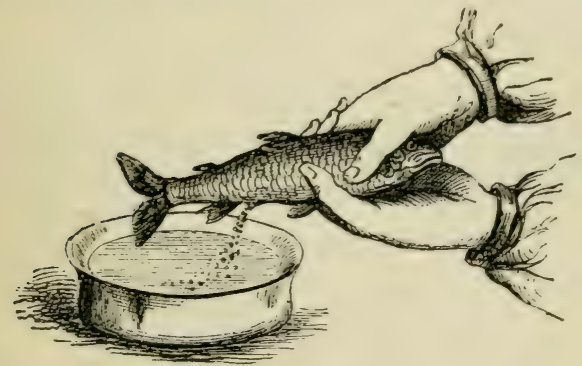

Fig. .3.

Il saisit un poisson femelle de la main gauche, le dos serré dans la paume, en le tenant aussi près que possible de la surface de l'eau, et arec le pouce de la main droite il exerce le long du flanc une légère pression qui fait sortir un jet d'ceuis (fig. 53). Ces ouf's plus lourds que l'eau tombent au fond du vase. En mème temps, un aide saisit le poisson màle et, en opérant de la mème manière, il fait écouler sa laitance en quantité suffisante pour donner à leau la couleur du petit lait. 
- Pour que la laitance impregne tous les coufs, on agite doucement leau arec la main ou mieux arec un pinceau à poils fins.

La féconuation est faite au bout de quelques minutes. A ce moment. l'eau troublée par la laitance a perelu sa qualité fécondante. On la rerse lentement et on la remplacepar de leau pure a la mème température.

Toute ses manipulations doirent se laire rapiclenient ef dans un endroit abrité contre le soleil et la lumière vive du jour. कै

\section{Appareils d'incubation. - Il siagit maintenant} doutenir l'éclosion des auls fécondés. On a inrenté pour cela des appareils plus on moins compliqués, très bons lorsqu ilssont destinés a des établissements rii la lécomblation artiticielle se fait sur une grande sehelle, mais 'fui ne sont pas à la portee des préposis. Le plus simple ef le plus efricace des appareils linsubation ron-iste en wne augette en terre rernie de so centimitres de long sur 1.j de large et so de fmofommlente dans laquelle on anlapte une raie en

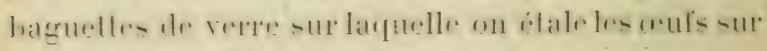
une couche ou deux au plus.

Lin filet deau regré par un robinet détermine un courant continu qui assure le dévelopjement régulier des wufs. Au moment de léelosion, les alevins passoul a travers lis baguettes de rerre ef lombent 


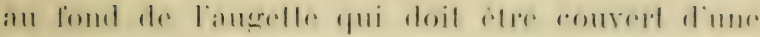
couche re sable fin.

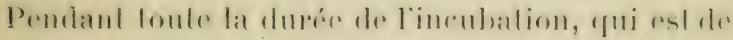
six semaimes a dems mois, il faul (mlever loms les

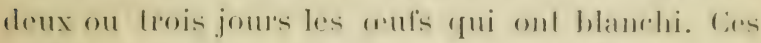

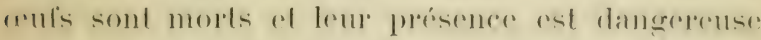
prom les antres. Pemband les premiers jomps, le repos lo plus eomplet est dabsolue necessile.

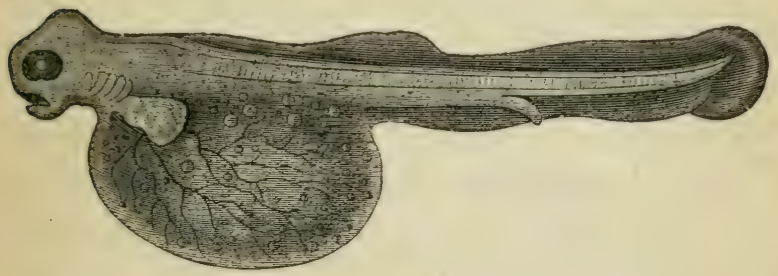

Fig.

A telaut diapratreils de celle nalure, on peut liare divelopper les aruls de sammons, de truites et lombres dans de pelits ruisseaux à fond caillouteux.

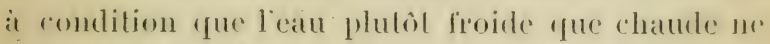
suit ni lres prolonde ni tris conrante et que tons les ennemis des ofuls et des alevins en soient soigneusement écartés.

Lorsque le petit poisson des espèces des Salmo.

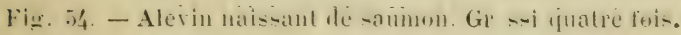


nides éclòt. il porte une énorme poche qui renferme les substances dont il se nourrit pendant son jeune àge (fig. 54). Cette résicule ombilicale très proéminente, condamne les jeunes alevins à limmobilité $\mathrm{t}$ les rend incapables de se soustraire à la roracité c'e leurs ennemis.

Ciest à ce moment que l'interrention de l'homme est la plus utile, car c'est celui où la destruction est la plus active.

Pour mettre les embryons sortant de l'wuf' a l'abri de leurs ennemis, il faut les conserver quelque temps dans les appareils d'incubation. On les y laisse pendant cinq ou six semaines, dans une demiobscurité et sans leur donner de nourriture, car ils se nourrissent arec les substances contenues dans la résicule ombilicale. Vers la fin de cette période. la résicule est résorbée; lalevin, devenu vif et agile. peut chercher sa nourriture. On peut alors le porter directement dans les cour's d'eau quil doit repeupler.

Il serait sans doute préférable dattendre que les jeunes poissons soient plus développés pour les mettre en liberté, mais il faudrait pour cela disposer de bassins dalevinage dans lesquels ils seraient conservés et nourris.

Les gardes qui ont à leur portée une fontaine d'eau vire et fraiche peurent en utiliser le bassin pour y élever des alevins de truites et de saumons: 
sils ont les mogens de les nourrir. Les aliments yui

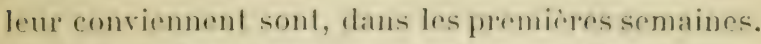
de la chair de poisson on de mollusques dean doure. brogée laes fin et passéc au tamis; plus tard, on pent lrur dommer des vers de vase, des latrves.

Les jeunes poiscoms ur mangent yur les alimruts

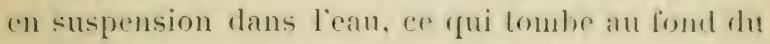
bassin nest pas utilise par eux. Il est important de. ue pas laisser ces résidus séjourner dans les bassins dont ils corrompent l'eau ; il est non moins important d'en extraire tous les alevins morts.

Lorsqüils sont devenus assez vigoureux pour chercher eux-mèmes leur nourliture, on doune aux alevins des crevettes et des cloportes deau douce puis, plus tard, des petils poissons des espèces herbirores : ablettes. loches, vairons, vandoises, etc., quion troure en abondance dans tous les cours dieau.

Quant aux poissons comme la carpe, la tanche, (qui se nourrissent dherbes, de graines, dinsectes et de rers, on leur doune des débris de cuisine, des pommes de terre, des détritus des jardins. Ces poissons sélèvent aisément dans des réservoirs de peu détendue où, aree quelques soins, on peut leup faire atteindre de tris fortes dimensions.

Lélevage du brochel nest gruere a recommander, ral ce poisson est tres votace; cepentant il niest pas inntile den laisser quelques-mus dans les cilangs, 
jarce qüil délıuil me grande: quantilé du lielin qui alsorbe la nomriture des carpes, mais il ne faut pas 'püil derienne assez vignoureux pour sattacfuer aux “arpes quion y élère.

Pammi les poissons doml la multiplieation est fitrile. uous citerons languille dont les alevins, éclos ('n mer, remontent les fleures en si grande quantité yuon peut les recueillir d̀ pleins seaux. Cés alevins, 1:0mous sous le nom de Montée, arrivent jusque dans l1 puisioau et los etangs oil ils se déreloppent rapidement. Iangmille se nomrit de rers, de frai, de

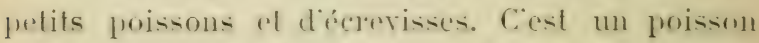

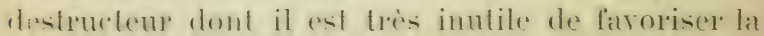

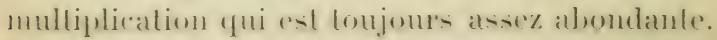

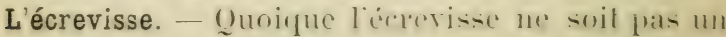

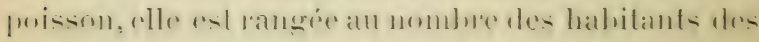

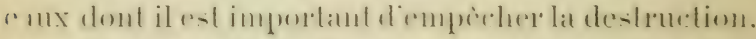
Iusi las arrolés qui reglemenlent lixereice de la prohe eosliennent-ils tous des disposilions contermant l'écrevisse.

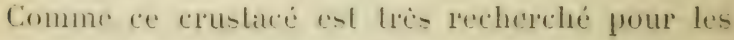
"falités de sa chair el yue la peche en est lacile. il I nd a derenir rape en France ou il élait aubelois

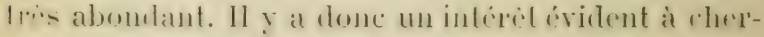

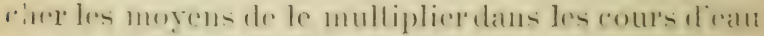

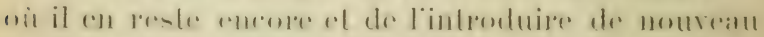
daus ceux doù il a disparu. 
Dams lo furemier cas il suflira dy inderdire rompla-

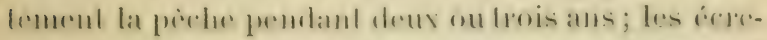
visses se reproduisent lateilement el se défemdent assez bien roulde leurs emmemis four que les comr-

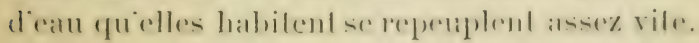

soil ny a plus dererevisses dans un ruissean oil il y en arait antrefois, le seul mogen matique de les y ramener comsiste à y porter, dans des panires a raire-roie très serrée, des femelle's chargées dirulis prèts d'éclore.

Les jeunes, à peine sorlis de l'orud, se moltenl a nager et sont bientòt en élat de chepcher eux-mèmes leur nourriture, mais il faut pour qüils sacelimatent que le cours deau remplisse certaines conditions : d'abord leau doit itre $u 11$ peu calcaire et.limpide: les berges argilenses permellant aux érerises dy reuser les trous où elles se logent. ce sont celles quelles prélèrent, mais elles se contenteut de relles qui sont formées de roches dont les interstices leur ofirent un abri. Les cours deau bordés daunes et de saules leur comvicunent micux que ceux dont les bords sont dénudés. Les écrevisses croissent lentement. Ce n'est que ver's la quinzième année quelles ont acquis tout leur développement; elles pèsent alors 100 à 1qo grammes. Elles se nourrissent de larves, de frai de poisson, de loules matières animales ef régétales rivaules on moptes. 
Parmi les nombreux ennemis de l'écrerisse, nous signalerons : les rats d'eau, les canards, les ràles d'eau, l'anguille. le héron. Les grenouilles et les tritons mangent beaucoup d'écrevisses dans leur premier âge.

Yous ne pourons donner ici que des notions très sommaires sur la pisciculture. Nous renvoyons ceux de nos lecteur's qui roudraient aroir des renseignements plus complets sur la fécondation, le transport des ceufs et des alerins, au traité de pisciculture publié par M. Pizzetta (1). Ce lirre, illustré de nombreuses grarures, contient tout ce qui est indispensable à un débutant pour obtenir de bonnes éclosions et mener à bien les jeunes alerins.

(1) Lat Pisciculture funtiale el muritime en France. par Jules Pizzetta, in-18, 212 gr. Libr. Rothschild. Pr. 4 fr. 
CHAPITRE XIV

DES CRIPTION

DES

PRINGIPALES ESSENGES

FORESTIËRES 


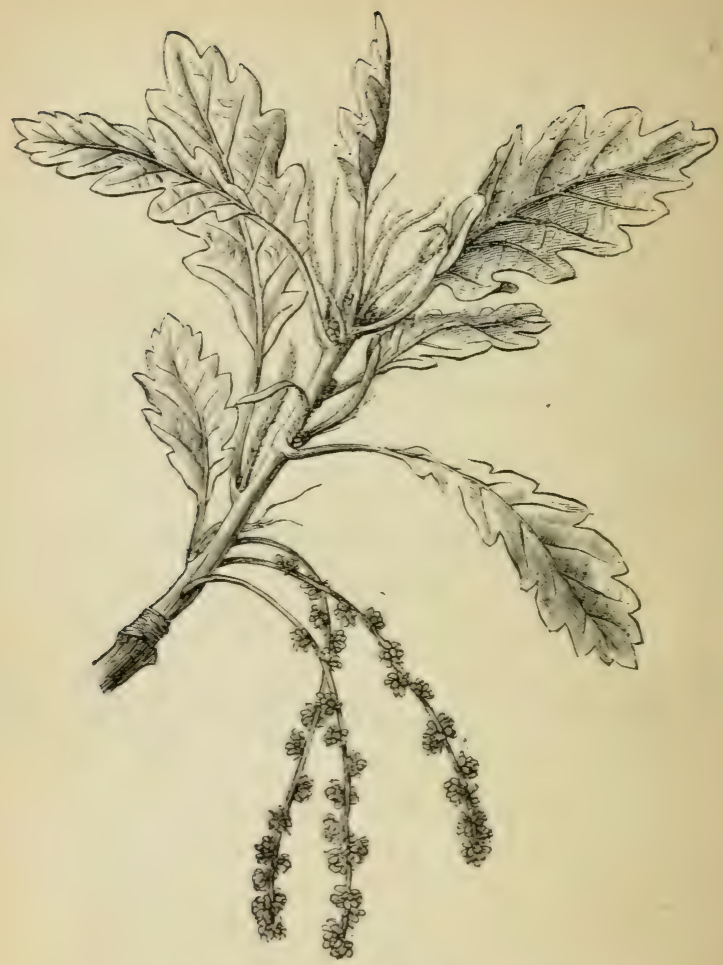

Fig. 5. - LE CHEXE RULTRE

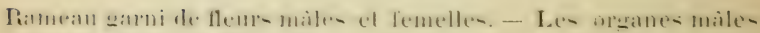

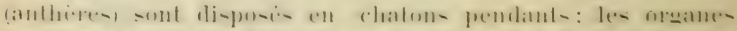

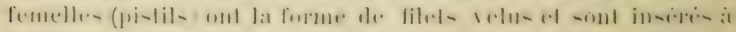
la base des feuilles. - Floraison, arril: malurite. octobre: dissemination, novembre-disembre. 


\section{Fir. ग. - LE CHENE ROUVRE}

\section{Gueren: Rubur}

I. chine rourre est un aldere des elimals tempieres. lessols qui lui conviennenl te mieux sont renx qui sont profonds, frais el substanliels, mais il croit anssi dans les terrains granitiques, siliceux ou raleaires.

sia longevite est tries grande. Il vit jusqu à 30 ans of

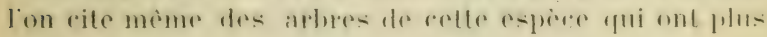
fle 6 ion ans.

Les jemes planls sont robustes: ils onl un pirol allonge qui sinfonce profondément dans le sol.

on enllive te rheme rompe en fulaie et en tailli-s.

Le couvert de eet arlore est liger. ()uand on le rullive en fulaie, sans mélange d'auleres essences, il niombrage pas assez le sol pom lempercher de se gazonner. On remilie i cet ineonrénient en lassociant an pin syestre et an charme dans los plaines et les montagnes peu élevées : à de plus granrles altiturles. on le rultive en mélange avec le hêtre et le sapin.

Le chrine est un arbre de lumiere: il ne supporte pas un état serré: il faut éclaircir de bonne heure les massils de cette essenee et élaguer peu ì peu le- plus beaux pierls pour rigulariser lem forme.

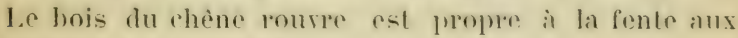
ourrages de menuiserie. 


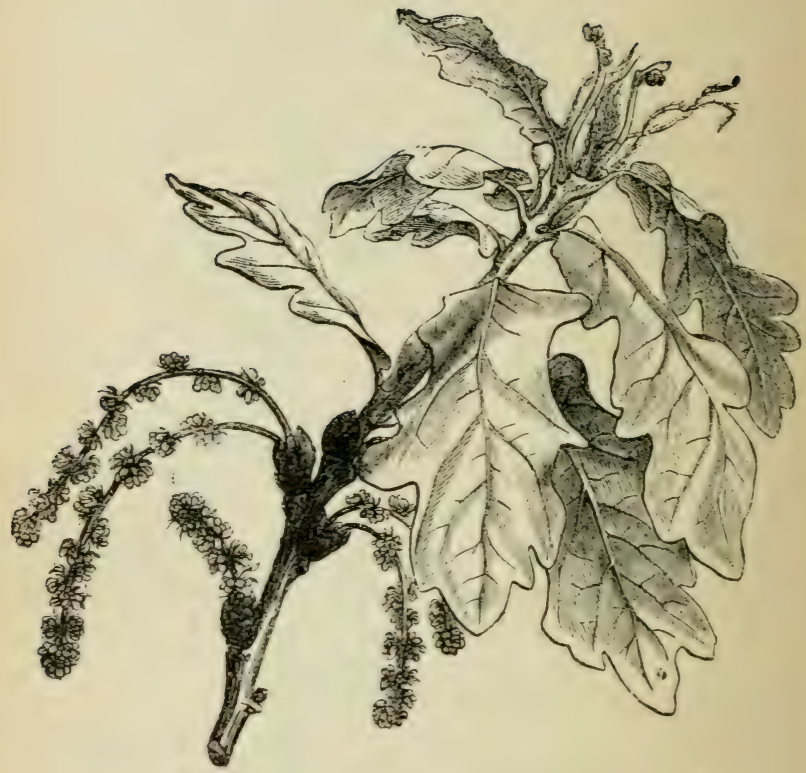

Fig. 56. - LE CHÊNE PÉdONCULÉ

Pamean gami de lleurs males et femelles. - Fenilles ses-iles. Glambs porté sur un pédoncule allonse. - Floraison. arril : maturiti. octobre: dissémination. novembre-décembre. 


\section{Fị. \%i. - LE CHÊNE PÉDONCULÉ}

(Quercus pedunculata)

Le chène pédonculé a les mèmes exigences que le rouvre; il est cependant un peu plus difficile sur le choix du terrain. Les sols argilo-caleaires ou argilo-siliceux sont ceux dans lesquels il acquiert les plus grandes dimensions et la meilleure qualité.

Le bois du chène pédonculé, plus nerveux que celui du rouve, est plus propre a la charpente el aux cons. tructions qu'à la menuiserie.

Ces chènes sont très propres ì itre traités en taillis. parce qu'ils repoussent bien de souche. Dans les taillis sous futaie, on les choisit de préférence comme bali. veaux à cause de la légèreté de leur couvert.

Lécurce du chène fournit le tan employé au corroyage des peaux, Les jeunes brins de taillis à écolce argentée produisent le tan le plus estimé.

La croissance du chène pédonculé est plus rapide que celle du rourre. Les jeunes plants sont robustes, ils dépérissent sous un couvert épais; pour les obtenir bien conformés, il faut semer les glands dans un terrain profondément ameubli et purgé des manvaises herbes. 


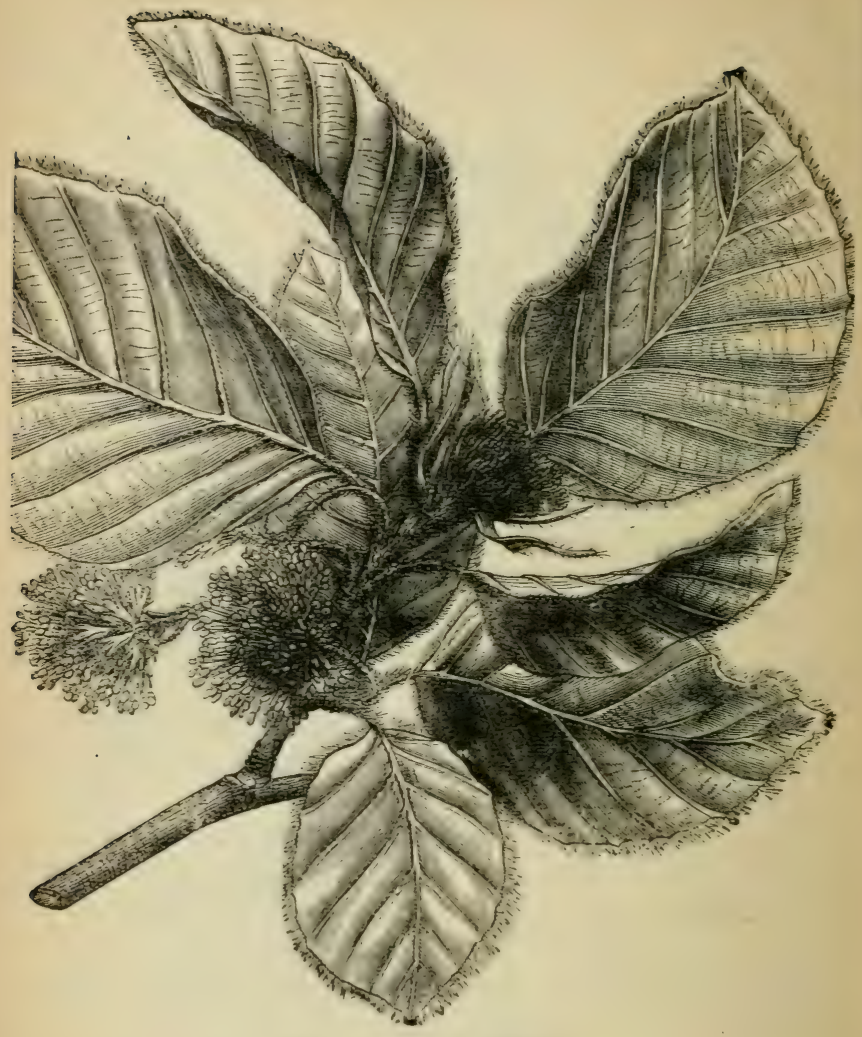

Figr. 5.7. - I.E IIÈTRE

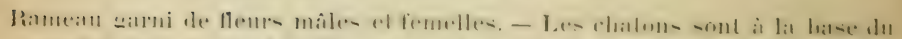

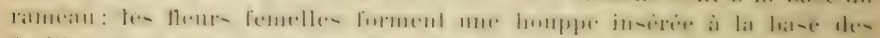

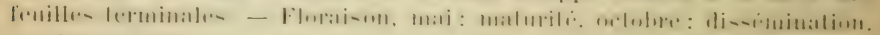
netobre-novembre. 


\section{Fig. 57. - LE HÊTRE}

\section{(Fagus sylualica)}

Le hetre rroit sur les coteaux et les montignes : les -limats humides ne lui sont pas défavorables: il redoule les sols sees et les expositions chaudes.

Ses racines trarantes lui permettent de eroitre ares vigueur sur des terrains pen profonds, mais il raind reux qui sont argileux et surtont marécagenx.

Le hetre envient surtont pour la futaie. Sil croissince, diabord tres lente. sarlire dies quil a alteint ligge de 1.t ans. Cet arbre eroit trè bien en mas-if : il donne un couvert compact.

Les jeunes plants sont tries délicats, ils craignent d'être exposés au soleil et doirent ètre blerís à lombre. () asiocie très arantageusement le hetre an rhene, au sapin et à l'épicéa.

Le hois de hetre est emplorí au chauflage : on sen sert aussi pour les ourrages de boissellerie el de tour. 1)epuis quelques années, on fait les traverses de chemin de fer arec du bois de hètre injecté. Cette industrie, qui a pris un grand développement, a fait recomnaitre 'que lélagage a pour effet de rendre impropres à linjection les portions des liges qui portent des cieatriens recourertes. 


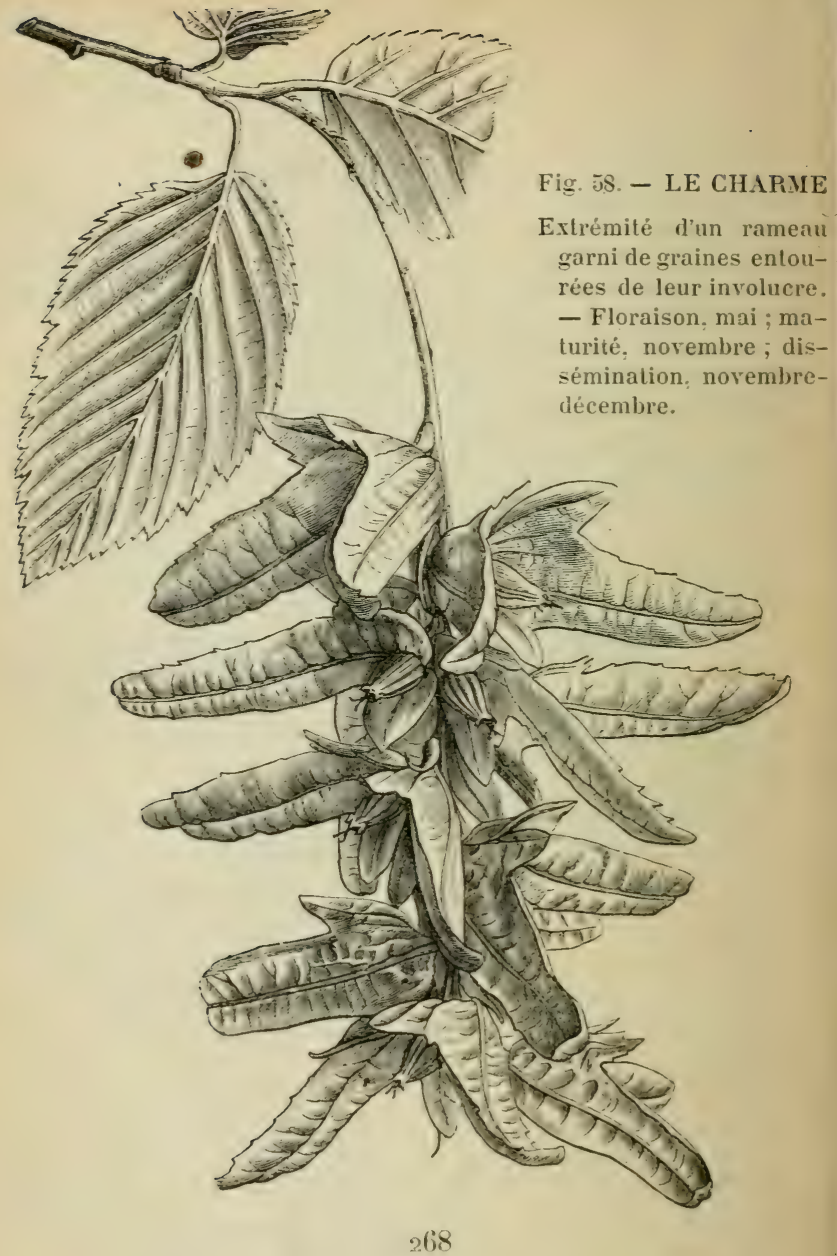




\section{Fig. 5S. - LE CHARME}

\section{(Carpinus: Belulus)}

Le charme est un arbre des climats tempéris: il habite de préférence les plaines et les montagnes de peu d'élévation. Les sols qui lui conviement le mieux sont cenx qui sont frais et profonds. Comme le chène, il cotédurgel on le rencontre souvent, il s'accommode assez bien deterrains argileux.

Le charme repousse bien de souche; aussi est-il habituellement exploité en taillis. Comme sa croissance est lente, les révolutions arloptées pour les taillis de cette essence sont d'au moins dix-huit ì ringt ans; traité en futaie, en mélange arec le chène et le hètre. le charme est considéré comme une essence secondaire destinée à favoriser la croissance des arbres plus précieux auxquels on lassocie. Il supporte bien le couvert.

Les jeunes plants sont robustes et la reproduction facile.

Cet arbre, qui supporte bien la taille, est employé a la confection des haies.

Le bois de charme est excellent pour le chauffage. On lemploie aussi à la fabrication des moreux, des dents de roue et à tous les ourrages qui demandent une grande ténacité. Il peut remplacer le chène pour les traverses. quand il a été préparé par les procédés d'injection. 


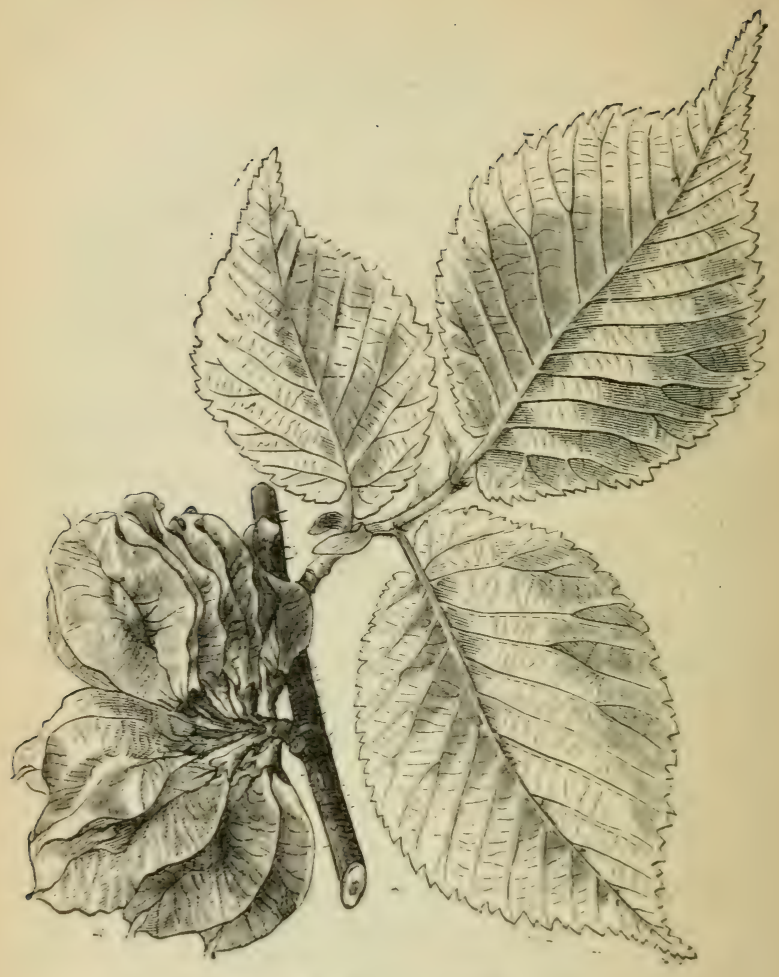

Fig. 5̆. - L ORME

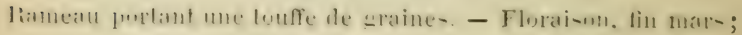
malurite. fin mai : dissemination. mi-juin. 


\section{Fir.}

\section{(Ulmus: cumpestric}

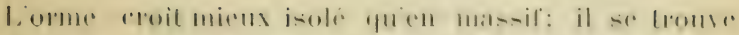
dissemine dams les forets. mais il ne derient jamais lies. sence dominante. Il aime les rlimals temprepés. lis suls frais ei divisés: il domme des rejels vigoureux el dral. geonne beausoup. On l'exploite en taillis. mélangé arer le chène, le charme et les érables.

Les jeunes plants sont robustes el lene eroissance esl tres rapirle: mais ils sont exigeants sous le rapport th sol, ils ne prospierent que dans los ferrains substantiels. bien ameublis et frais.

l.a fructitication de lorme es lies abondante. Le graines, pourves d'une aile membraneuse, se disséminent vers le milieu du mois de juin. Ciest à celle éporpü qu il faut les recueillir pour les semer de suite, rar elles se conservent peu.

Le bois de lorme est thés bon eomme combuslible. mais on nemploie a cet usage que les branches el les liges délectueuses. car les pièces saines sont tris recherchées pour le charronnage.

On distingue plusieurs especes divmes : lo champelre, qui est le plus répandu; le pédonculé. 'qui diffère du précédent par la longueur du pétiule des feuilles, el le fongueux, reconnaissable à la petilesse de ses feuilles cet aux cxeroissances qui rourent les jeunes branches. 


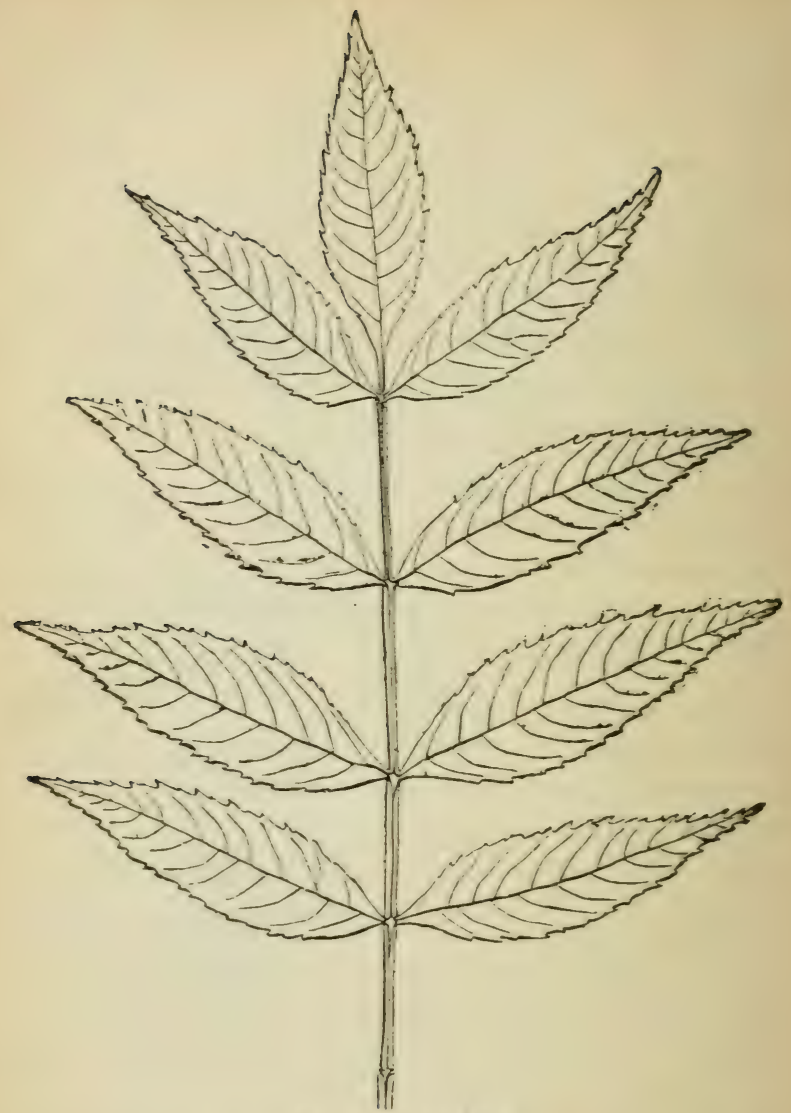

Fig. 60. - FRÈ̃E

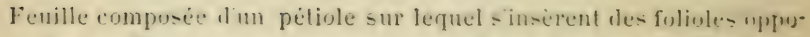
sés. - Florai-un. mai : maturite. octolne: dis-éminalion. hiver. 


\section{Fig. Go. - LE FRE $\mathbf{E}$ E}

\section{(Fraxinus excelsior)}

Le freme se troure dans les planes ansis bien fure dans les montagnes, où il sobleve à de grandes altitudes: mais partout il recherche les sols frais, profonds et divisés. Il redoute les expositions chaudes, les sols secs: aussi, dans les monlagnes, ne le rencontre-t-on que sul' les points où la terre est meuble et substantielle. Il est commun dans le voisinage des ruisseaux.

Cet arbre vit plus volontiers a l'état isolé qu'en massif', mais il s’associe très bien à l'érable, à l'aune, et. dans les montagnes, au sapin et à l'épicéa.

Les jeunes plants sont robustes; ils redoutent cependant les gelées printanières et l'envahissenent desherbes.

Dans les taillis, le frène s'associe tries bien aver l'orme, les érables, l'aune et les bois blancs; mais lorsqu il est coupé jeune, son bois n'a pas toutes ses qualités. Cultivé en futaie et surtout comme arbre de Jor dure, il produit un bois très recherché pour le charron nage et la menuiserie.

On traite aussi le frène en tètard, pour utiliser ses feuilles comme fourrage. Les troncs ainsi émondés tous les ans se courrent de nodosités qui fournissent des loupes très recherchées pour le placage. 


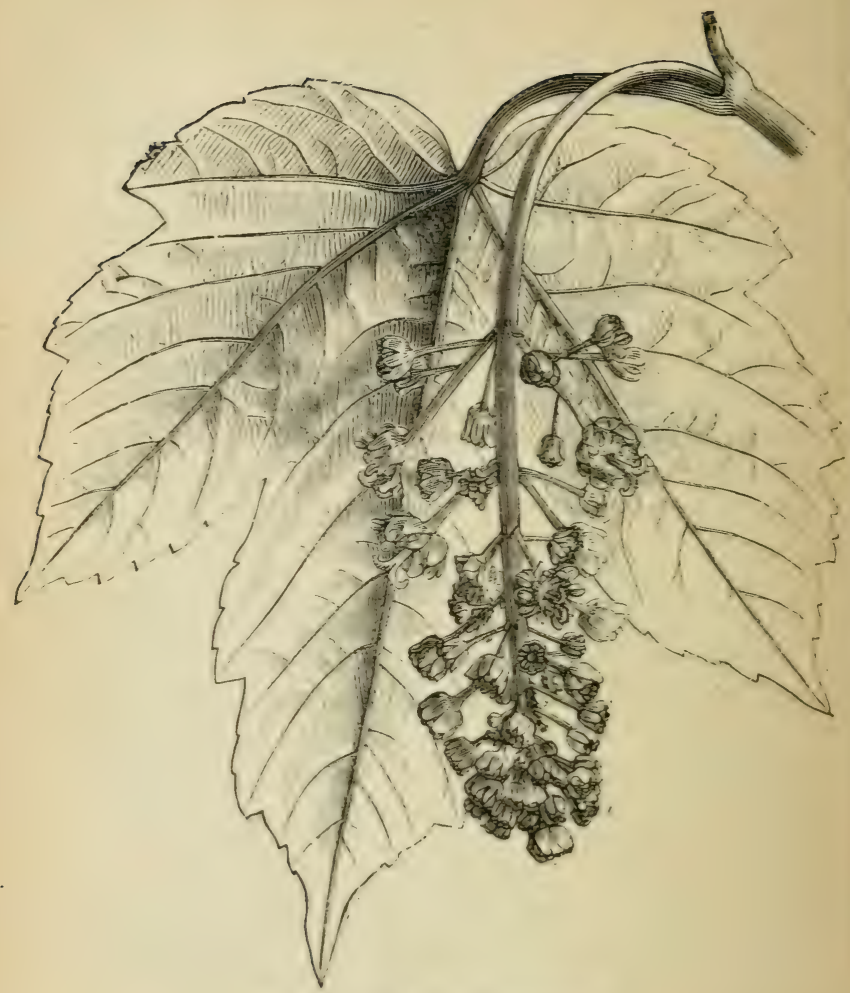

Fig. 61. - LE SYCOMOIRE

Rameau garni diune grappe de fleurs. - Foraison. mai fructilieation, octolne: dis-imination immérliate. 


\section{1iit. (1). - LE SYCOMORE}

\section{(Arer pletenus)}

Le sromore rst un arble des montagnes. Il croil aux mèmes altitudes que le hètre et le sajpin. aver lequel on 1e trouve souvent associé. 11 aime les sols frais et divisés. Cel arbre est disséminé au milieu des autres essences, mais il est rarement dominant. Cullive en mélange aver le frène et lorme, dans de bons sols. il acquicit de très belles dimensions.

Le syeomore est apte a supporter le rigime du tailliaussi bien que celui de la futaie.

Les jeunes plants emoissent rapidenent, el pourvu 'fu'on les abrite un peu dans les preniers temps de leur naissance, ils supportent très bien la chaleur.

Le bois de sycomore est assez recherché pour la menuiserie, le tour. le charronnage el la sculpture. Il a le grain fin et prend un beau poli. 


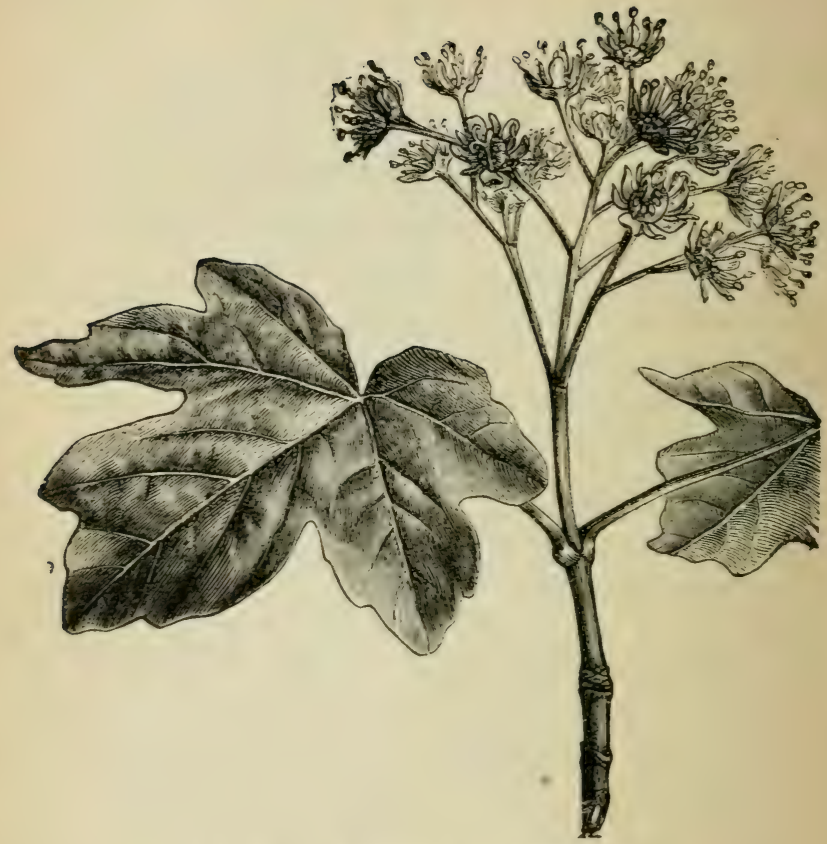

Fig. 62. - L ẺRABLE CHAMPÊTRE

Ramean garni de fleurs. - Floraison, mai; maturite, uclobre: dissémination immédiate. 


\section{FiE. 6\%. - L'ERABLE CHAMPÉTRE}

\section{(Acer cumpestre)}

L'érable cliampetre a les feuilles petites; ses jeunes pousses sont recouvertes d'une écorce rugueuse, sillonnée de côtes saillantes. C"est un arbre qui atteint rarement une hauteur supérieure à 10 mètres. On le trouve très souvent dans les haies et les bordures. Sa croissance est lente.

L'érable clampètre n’est pas, à proprement parler. une essence forestière. Cependant il est assez répandu dans les taillis, et comme il repousse bien de souche et qu'il śe produit aisément de graine, on doit chercher à le propager.

Le bois de l'érable champetre est rarement d'assez grande dimension pour ètre employé aux travaux de menuiserie, mais on lemploie aux ourrages de tour. Il est très bon comme combustible.

Dans les montagnes, on utilise les feuilles de l'érable comme fourrage. 


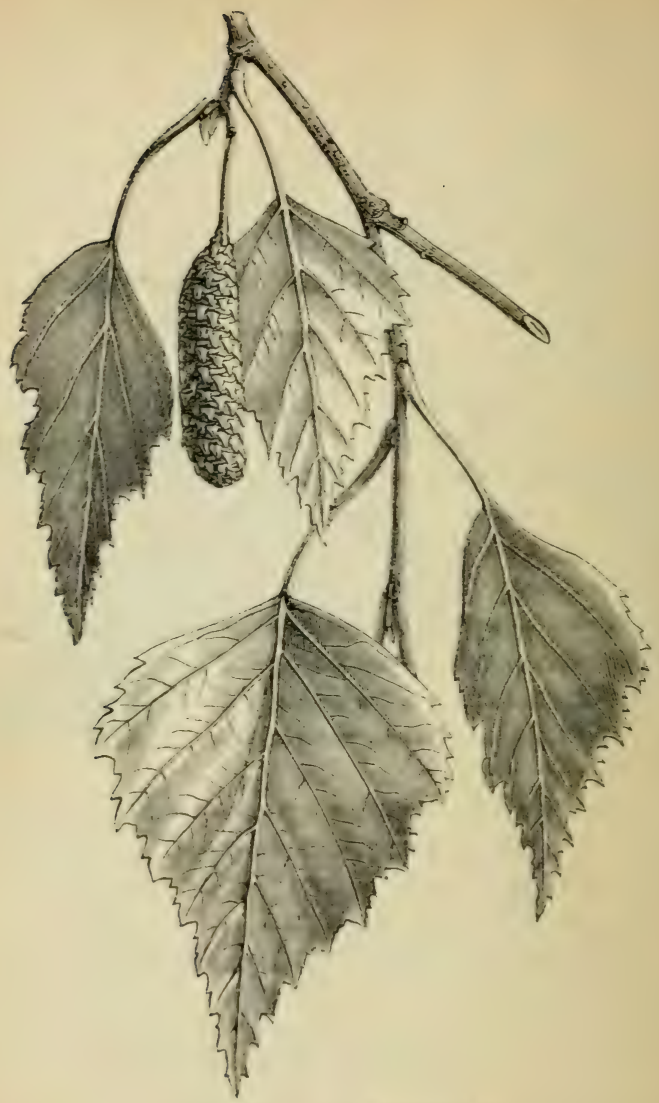

Fig. 63. - LE BOLIEAL

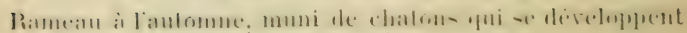
au printemps. - Floraison, mai : maturite. 15 septembre. 


\section{lii. 6.3. - LE BOULEAU}

(Belule allor)

Le bouleau est un des arbres les moins exigeants sous le rapport du sol et du elimat. On le trome sur les haules montagnes of dans les plaines des rigions sepptentrionales. C"est lessene qui supporte le mieux le froid. Elle saceommode de tous les terrains, pourvu quils ne soient pas trop comparts. Son fenillage liger abrite peu le sol et le laisse se courrir d'herbes.

Le bouleau ne doit pas citre eullivé i létal mur. mais il eroit très bien en mélange avee le ehrue el le pin sylrestre.

La graine, très fine, se dissémine en septembre: elle se conserve très facilement.

Traité en futaie, le bouleau s'exploite de fon a 80 ans: passé cet àge, il dépérit. On le cultive aussi en taillic; mais il donne des rejets peu vigoureux.

Les jeunes plants sont robustes et très abondants. fiest une des rares essences quion peut planter aree de jeunes sujets pris dans les bois.

Le bois de houleau est surtout employé an chauffage des fours. On se sert des jeunes brins de taillis pour faire des cercles et des ramilles pour la fabrication des balais. Dans beancoup de pars, cette industrie est entre les mains de maraudeurs qui aiment mieux voler la matière première que l'acheter. 


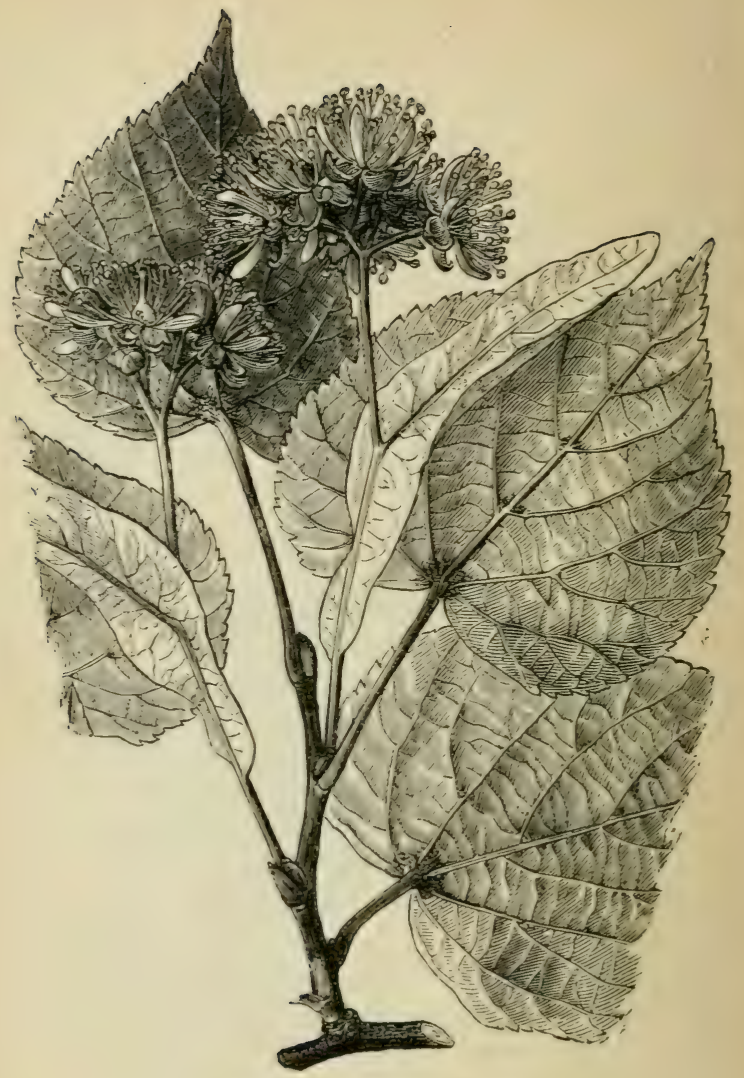

Fi世. $\sigma_{1}^{\prime}$. - LE TILLEUL

Pamr:ul anrni de fleurs hermaphrodites. - FIoraisnn. juin : maturite, septembre. 


\section{Fig. 6\%. - LE TILLEUL}

\section{(Tilia macrophylla)}

Le tilleul est un arbre des climats tempérés. mais il sélève à d'assez grandes altitudes. Les sols qu'il préfère sont ceux à base calcaire qui sont frais et divisés. On le Irouve cependant dans les roches fissurées et les sables granitiques ou siliceux.

Celte essence n'est jamais dominante dans les peuplements. Elle s'associe volontiers aux érables, au frène et a l'orme. On la trouve assez souvent dans certains taillis croissant sur des terrains humides.

Les jeunes plants ont à craindre la gelée et la sécheresse pendant deux ou trois ans; après ce temps, ils sont très robustes et se transplantent aisément.

Le bois de tilleul est léger, tendre et n'est hon ni pour le chauffage ni pour le travail; on ne peut s'en servir que pour la sculpture et quelques ourrages de trainerie. L'écorce des brins de taillis est recherchée pour la fabrication de liens; celle des arbres plus àgés sert à la confection de cordes a puits et de nattes très solides. 


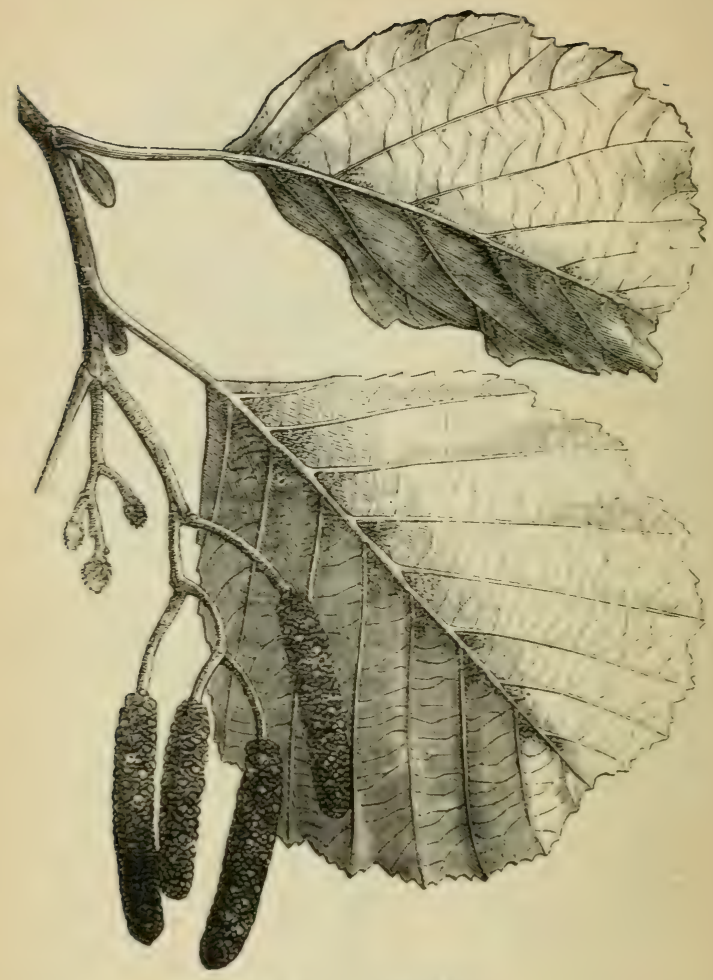

$$
\text { Fier. 6.) - I IITXE }
$$

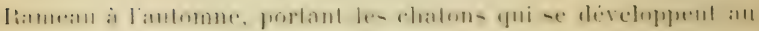
prinlemps, - Floraison, mars : malurile, nelobre. 


\section{Fig. 65. - L'AUNE COMMUN}

\section{(Alnus glulinosa)}

Lame eroit de prefipenee sur les bords des miscranx, dans les bas-fonds. Comme le courert de son fenillagr

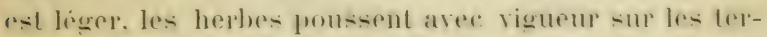
rains peuples de celle essenere qui exclut toules les autres, à l'exception du frène.

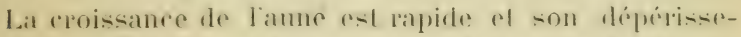
ment prompt. Il ne deprasse guere fo ans en linlaire, el, traite en taills. il doil elre coupe it 20 ans au plus. On propage laune. soil an moyen de boutures, soit par des semis. Quand on emploie ce dernier mode. il fant avoir soin de semer slir un sol plutit frais que sec. Les jemeplants sont robustes al peurent te passer diabri.

Liane donne un bois assez médiore comme rombustible, el impropre a la rharpente, parce quil so pirque. On lemploie a la enfection des sabots. des turaux de conduite et surtout. comme bois de tour, pour la confertion des chaises eommunes. Il s'en fait une grante ronsommation is Paris, pour ce dernier usage. 


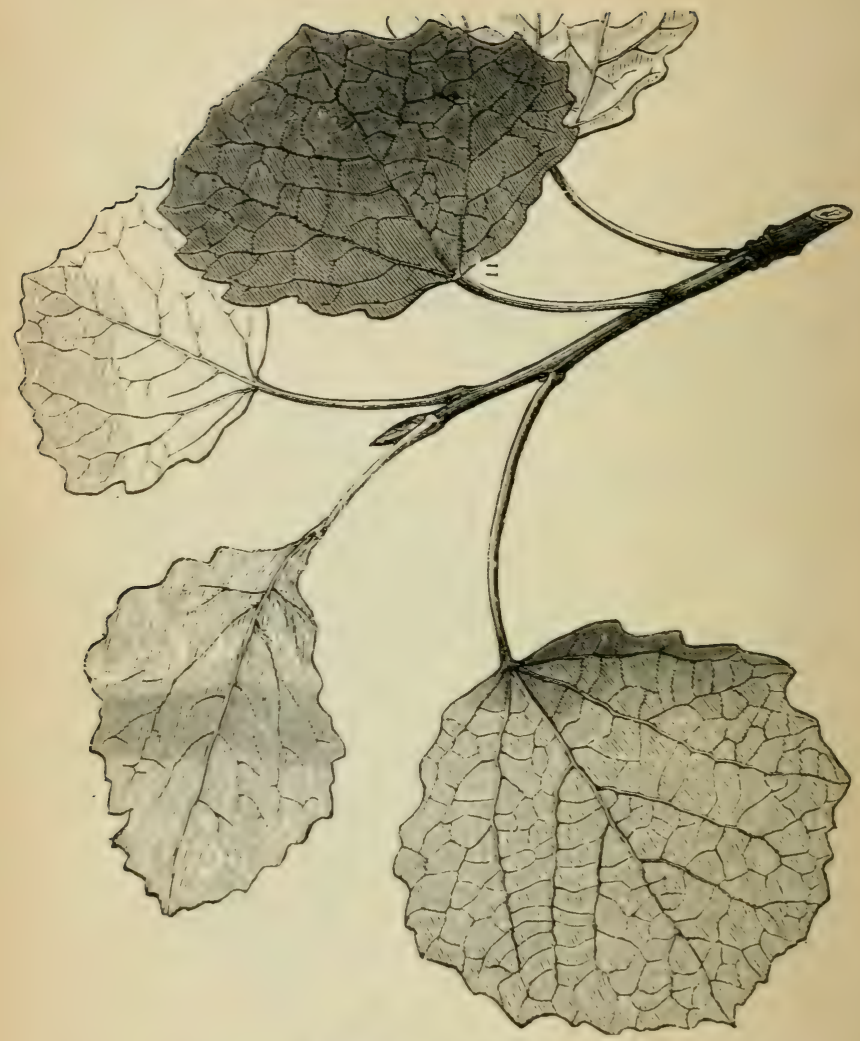

Fig. 66. - LE TREMBLE

Pomeau garni de ses fenilles altachées à de longs peilioles. - Floraison. arril: maturité, mai ; dissémination, mai. 


\section{Fi. 66. - LE TREMBLE}

\section{(Populus tremula)}

Le tremble est un arbre tres robuste quion trome dans les plaines temperées aussi bien que dans les régions froides et élevées. Il n'est pas difficile sur le choix des terrains, mais il préfère ceux qui sont frais et légers. Sa croissance, rapide dans les premieres années, sarrète vers làge de jo ans, éporque où il commence à dépérir.

Le tremble drageonne beaucoup; ses semences fines et abondantes se dissémirent au loin. 11 n'est pas rare de voir cette essence envahir les bois dont le sol est hunide et arrèter la croissance des arbres les plus précieux. On remédie à cet envahissement en pratiquant des nettoiements et des éclaircies.

Les jeunes plants sont robustes, leur reprise facile. On n'a d'ailleurs pas à s'in quiéter de la reproduction de cet arbre, qui se propage spontanément plus qu'il n'est nécessaire.

Le bois de tremble est employé au chauffage des four's. On en fait des caisses d'emballage et des voliges pour' la menuiserie. Depuis quelques années, ce bois a acquis une assez grande valeur dans les pays où il existe des fabrirques de pàte à papier, parce qu il est très propre à cette industrie. 


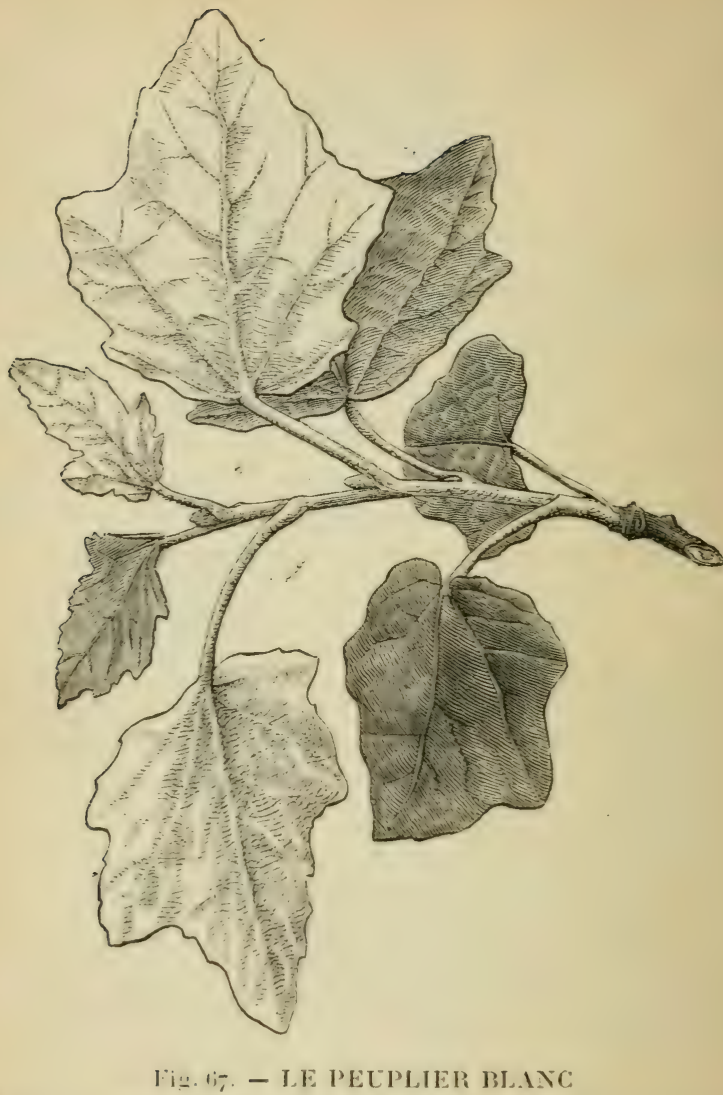

Ramean garni de [enilles vertes en dessus, d'un blanc cotmuneux en dessons. - Floraison, arril: malurile, mai : dissiminalion, III:ii. 


\section{lii. i- LE PEUPLIEF. BLANC}

\section{(Populus argentert)}

Le peuplier blane se distingue par se feudles luisantes a la face supréreme, drun blanc cotomeux en destons comme tous les peupliers. il aime les sols légers at frais. On le trouve habituellement sur le bord des ruisseaux, dans les prairies humides.

Cet arbre est rare en forct. et quand on l'y rencontre. cest dans les parties humides situces sur le- lisieres on le bord des fossís.

Emplové comme arbe dornement, te peuplier blans: est d'un très bel effet a cause de lopplo-ilion des contleurs de son feuillage. Il se détache heureusenent sur les masses rertes formées par les autres esseners.

Les usages du bois du peuplier blanc sont les mèmes que ceux du tremble. 


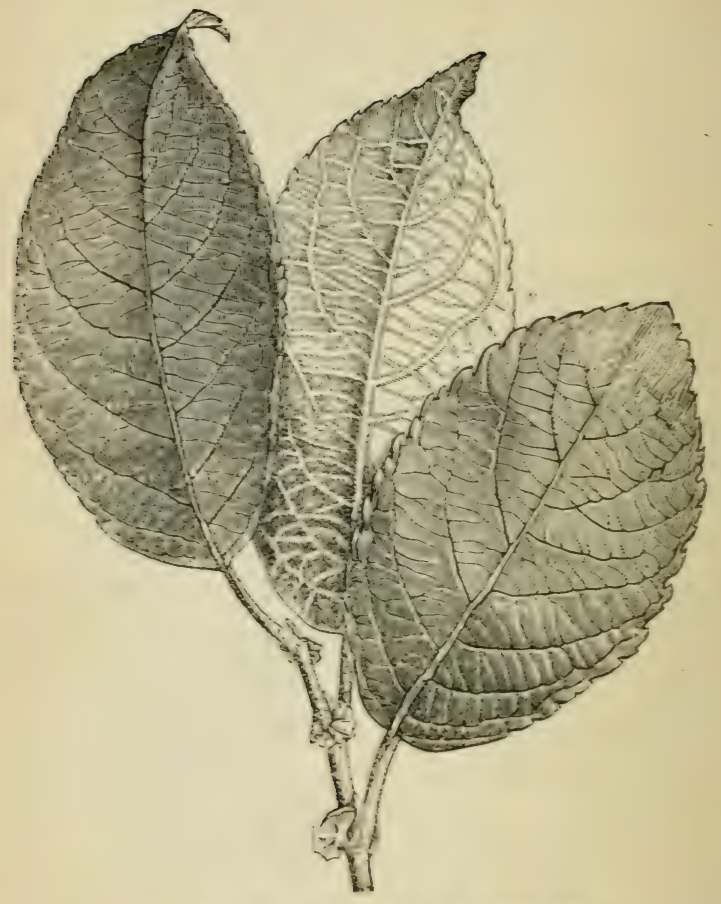

Fis. RS. - LE SALLE MARCEAU

Floraison, mars-avril: fruclification. mai : dissémination immédiate. 


\section{Fig. fS. - LE SAULE MARCEAU}

\section{Salix cupren}

Le saule marceau est. de tous les saules. le seul qui soit exclusirement forestier. Il est tres sourent associe, an tremble et recherehe comme lui les lerrains humides, quoiquil s'accommorle à peu près de tous.

Le marceau se reproduit tres aisement par ses graines. ses rejets el ses drageons. Ciest une essence enrahissante qui ne supporte pas le couvert et qui étoufte luns les plants qu'elle domine.

Sa croissance est rapide et son dépérissement prompt. Il est rare de trourer des sujets àgés de plus de jo ans.

Le bois du saule marceau est peu estimé comme comhustible. Les cultivateurs recherchent les tiges un peu fortes pour les refendre et en laire les dents de leurs ràteaux. 


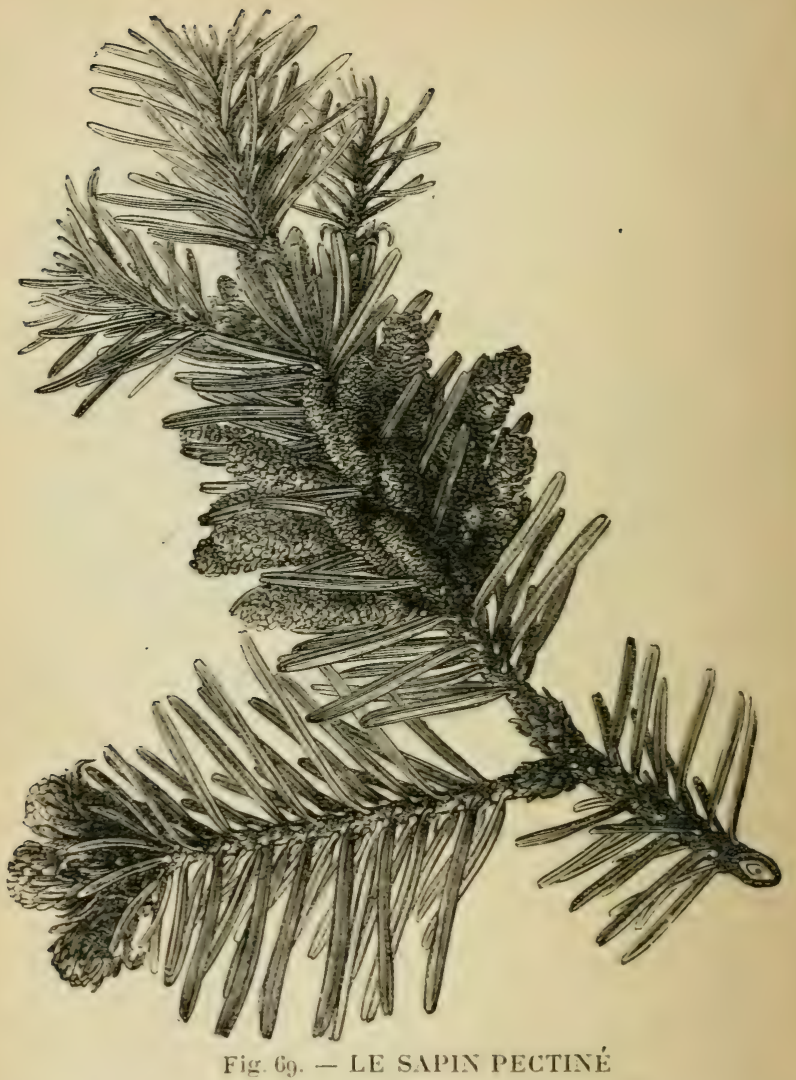

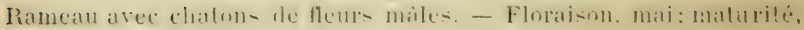
septembre: disseminalion. octobre. 


\section{Fir. big. - LE SAPIN}

Abies peclinalu)

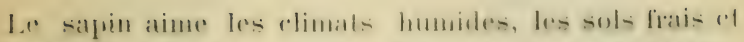
divisés.Il habite plus volontier- la montagne yue la plaine, mais il ne seleve pas i de lries gramles altiludes. Eu general. cet arbe a les memes exigences que le heitre avec lequel il est tries souvent ansoric. Les comes du sapin sont dressés, ils souvrent vers le commen:ement doctobre; les écailles et les graines tombent en meme temps.

On exploite le sapin en fulaie pleine ou en jartinant. Il se prete également à ces deux modes de trailement.

Les jeunes plants sont triss sensibles a la grelée et aux eflets du soleil, ils demandent à croitre à labri, aussi faut il serrer beaucoup les coupes d'ensemencement.

Les semis naturels réussissent bien. surtout quand la coupe est garnie de hetres trainmts: il fint don: se garder de trop nettoyer le sol des sapinieres.

Ouand on est ublige de recourir aux repeuplenent:artificiels, il raut mieux planter des sujets repiqués que d'essayer des semis, dont la réussite est loujours douteuse.

Le buis de sapin est employé à la charpente, a la menuiserie. 


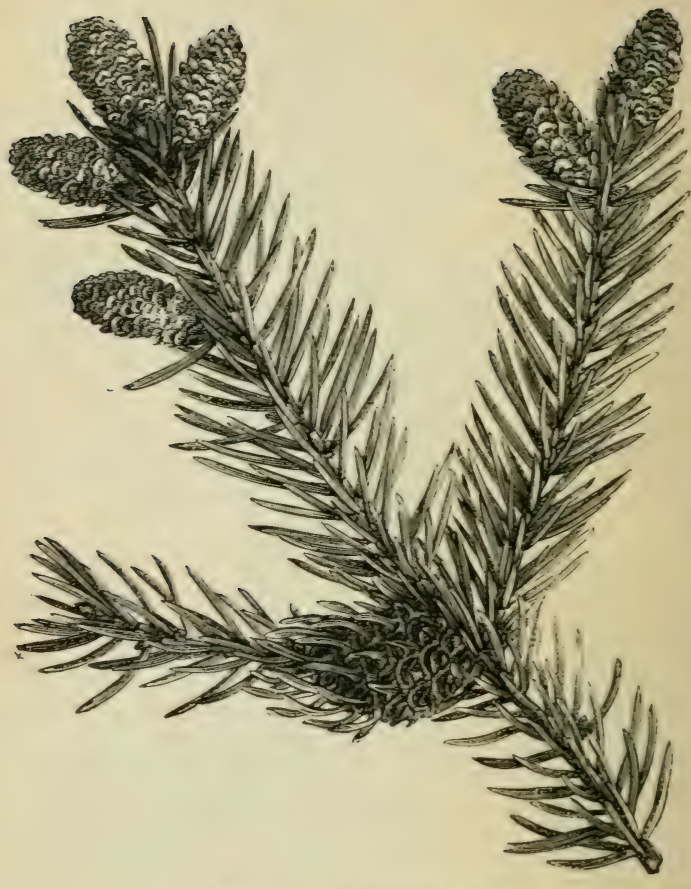

Fig. 7o. - L'ÉPICÉA

Ramean avee chatons males. Lexcrois-ance qui se trouve a la ba-e du rancan sterile e-t une galle produite par la pigure d'un insecte. 


\section{Fig. 7o. - L'ÉPICEA}

\section{(Abies Picea)}

Lépicéa est moins difficile que le sapuin sur ie chroix du sol, mais il préfére ceux qui sont frais.

Ciest un arbre de montagne quion troure somwent milangé avec les hetres. les érables el les chènes.

Ses cones sont pendants; ses feuilles, moins larges que celles du sapin argenté, sont plus épaisses et 11 ont pas, comme ces dernières, deux lignes blanches it la face inférieure ; elles sont presque aussi épaisses que larges.

On exploite l'épicéa par la méthode de la futaie pleine. qui réussil assez bien quand les réserves des roupes d'ensemencement ne sont pas renversées par les vents. Dans les contrées où ces accidents sont à craindre. on exploite à blanc-étoc et l'on repeuple artificiellement.

Le jeune plant est plus roluuste que celui du sapin: il a besoin d'un léger abri pendant les premières années mais il croit très rapidement dès que le couvert est formé. On doit se garder de constituer des massifs purs d'épicéas dans les sols maigres ou épuisés. Ces peuplements. après avoir régété pendant quelques années, s'étiolent; les insectes s'y jettent et les dérastent. Dans de pareils terrains, il faut mèler à lépicéa le hètre, le sapjin el au besoin le pin strlvestre. 


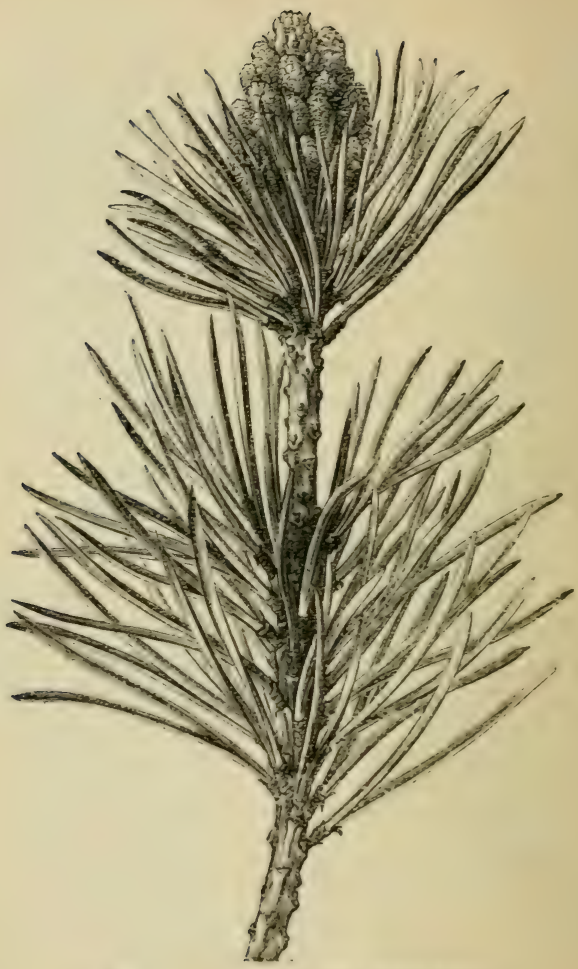

Fig. 71. - LE PIN SYLVESTRE

liamenu aver ses chalons de lleurs mates. - Floraison. avilmai : malurile. octolore de la seconde année: dissémination. priulemps -nivant. 


\section{FiY 71,- LE PIN SYLVESTRE}

\section{(Pinus sylueslris)}

Co pin sacommode de presque tous les terrains: an 1r. Irouve dans les plaines aussi bien que dans les monlagnes. Les sols quil parail preferer sont reux yui

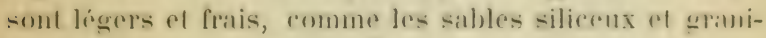
tirques.

Les eones, qui eommeneent a se former ans-ilit apuila fécondation, éest-il-dire rers le mois de mai, ne soml mùrs quau mois dortobere de lannep suivante. I.re graines se disséminent au printemps.

Le pin sylrestre croil tress rapidement et vil justü zox ans: mais il est exploitable rers go ans.

lelle essenee sexploite en coupes régleses el mome it blancétoc. Dans des conditions favorables. lensenenrement naturel se fait aiśment au moyen des graineapportées par les rents. Le jeune planl est robustre il n'a besoin d'aucun alori.

Le pin sylvestre est un arlore precieux pour lo leppenplement des sols maigres ou ipuisés. quil ambliore an peu d'années.

Le bois du pin sylrestre semploie aux mèmes usagus yue celui de lépicía. Il entre dans le rommeres somle nom de pin rouge du Nörd. 


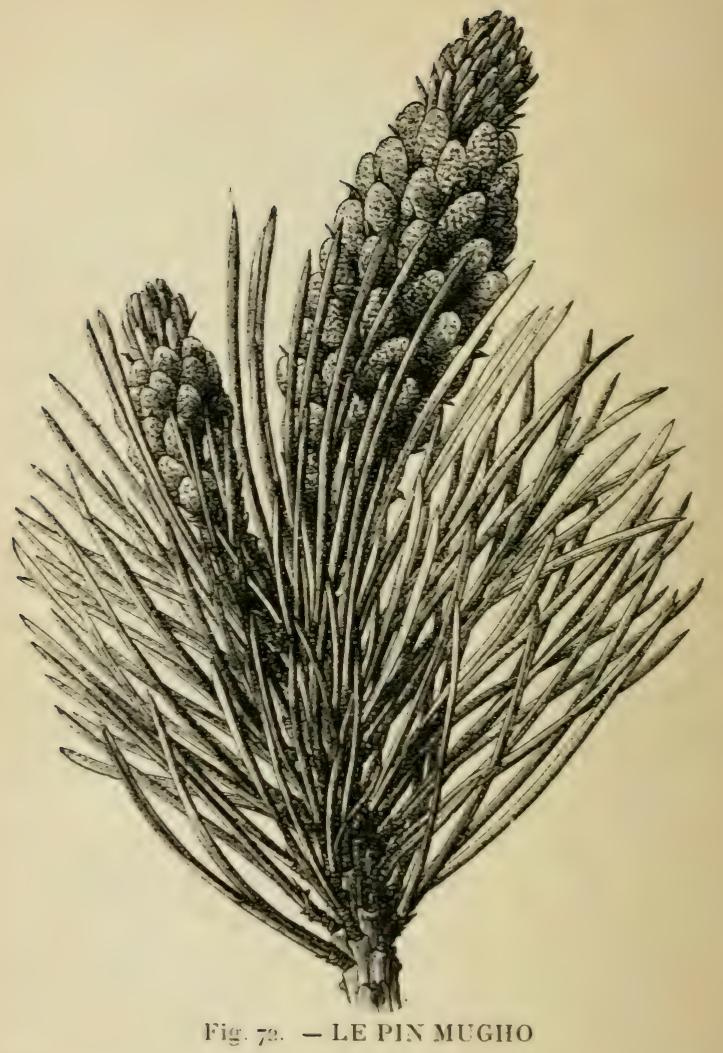

Pameau arec chatons màles. - Floraison, mai-juin ; maturité, vetube de la seconde annee : dissémination \{mai suivant. 


\section{Fig. 7\%, - LE PIN MUGHO \\ (Pinus Mugho)}

Le mugho, appelé aussi pin de montugne. torrhepin, ressemble beaucoup au pin sylrestre, mais ses aigruilles sont plus courtes; ses cones, plus petits, pendent aux branches et sont recourbés vers le sol.

Le mugho croit sur les haules montagnes, vers les limites de la régétation forestiere, et se troure a l'étal darbuste dans les paturagres élevés, sur les terrains tourbeux et marécageux.

L.e mugho, dont la constitution est très robuste. résiste à tonles les intempéries. C'est une essence pricieuse pour fixer le sol sur les versants élevés où ancune autre essence ne peut prospérer.

Son bois ua de valeur que pour le chanffage. 


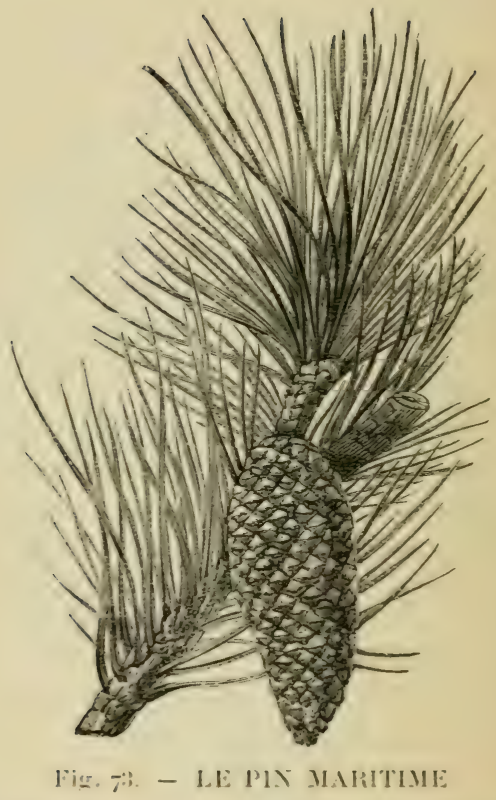

Iiamean avec un cone. - Floraison, mars-arril : malurile, of-

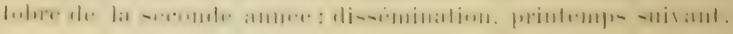




\section{Fiv 73 - PIN MARITIME}

\section{(Pinus Pinusler)}

Le pin maritime habite de preférence les contres roisines de la mer. On le troure anssi dans les plaines du eentre de la Finnee mais eomme il pedoule bean(onip) lat gelée, il y alteint rarement de belles diment sions. La semence mel deux amóes a murir, elle se dissimine en mai. Croissant sous m elimat tries rimulier, le pin maritime produit des graines prestgue tous les ans : aussi sa reginefation est-elle facile.

f:est avee re pin duton est partenu a fixer la grande bamde de sable qui longe les robles de locéan depuis Bordeaux jusquà Bayonne.

Les jemes plants sont assez robustes pour se passer diblori, mais quand on les emploie pour fixer les sabledes dunes. on est oblige de faire les semis sous me couverture de hranchages i moitié ensablés. Les graines germent sous ees abris, ol les jemes plants. passant it travers leurs interstices, ont lientiol courert et fixe le sol.

Le hois du pin maritime sert a faire des étais de mines, des traverses de chemin de fer. Cet arbre est en général traité en vue de la production de la résine. Dans les pars du centre, ou le gemmage est peu lueralif, on fait aree les jennes pins du bois de four, dont l'emploi est considérable. 


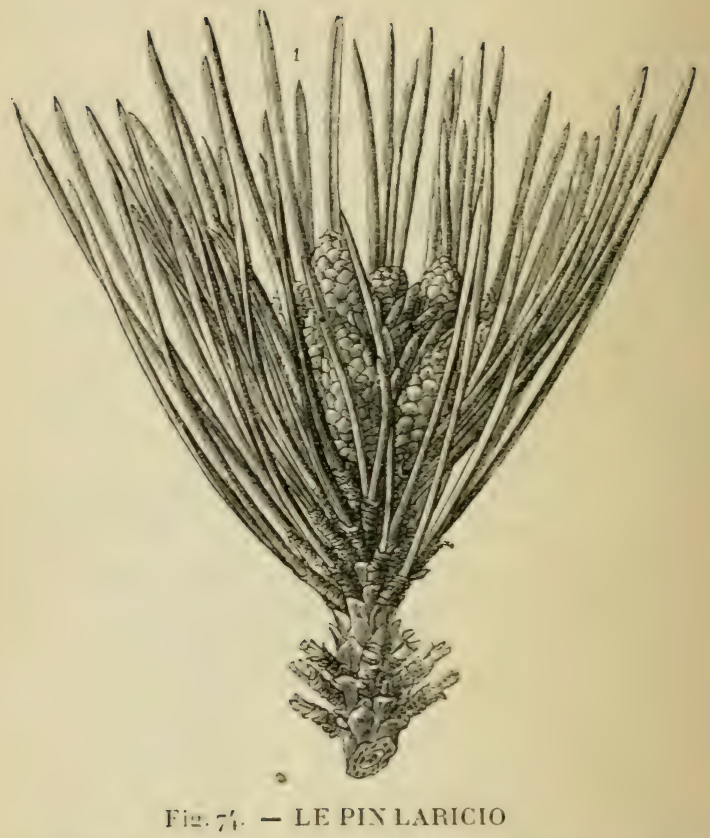

Jeune pousse avec chatons màles, - Floraison. mai : maturité. octobre seconde année: dissémination, printemps suivant. 


\section{Fig. 准- LE PIN LARICIO}

\section{(Pinus: Laricio)}

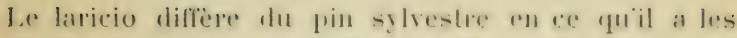
pousses plus vigoureuses les aiguilles plus longues it pius lortes, les cònes plus grands. Son temperament est robuste, sa croissance rapide. Il sexploite comme ce dernier.

Lat variete connue sous le nom de pin noir al . lulriehe est remarruable par sa vigueur el par la facilite aver latquelle elle croit sur des sols de qualité inférieure. Les terrains calcaires secs, qui conviennent à très peu d'essences, ne lui sont pas aussi défarorables que les fouds argileux et humides.

La culture du pin laricio est la mème que celle du pin sylrestre; ses jeunes plants se passent de tout abri. Les racines, très traçantes, s’étendent au loin.

Le pin noir a la tète fournie: dans les contrées muntagneuses, ou les neiges sont abondantes. ses branches sont souvent brisées. Si le sol est peu profond, larbre tout entier est sourent renversé par le poids des neiges qui saccumulent sur son épais feuillage.

Le bois du laricio est très bon pour la charpente. On extrait aussi de cet arbre une résine qui sert aux mèmes usages que celle du pin maritime. Les procédés d'extraclion sont à peu près les mèmes. 


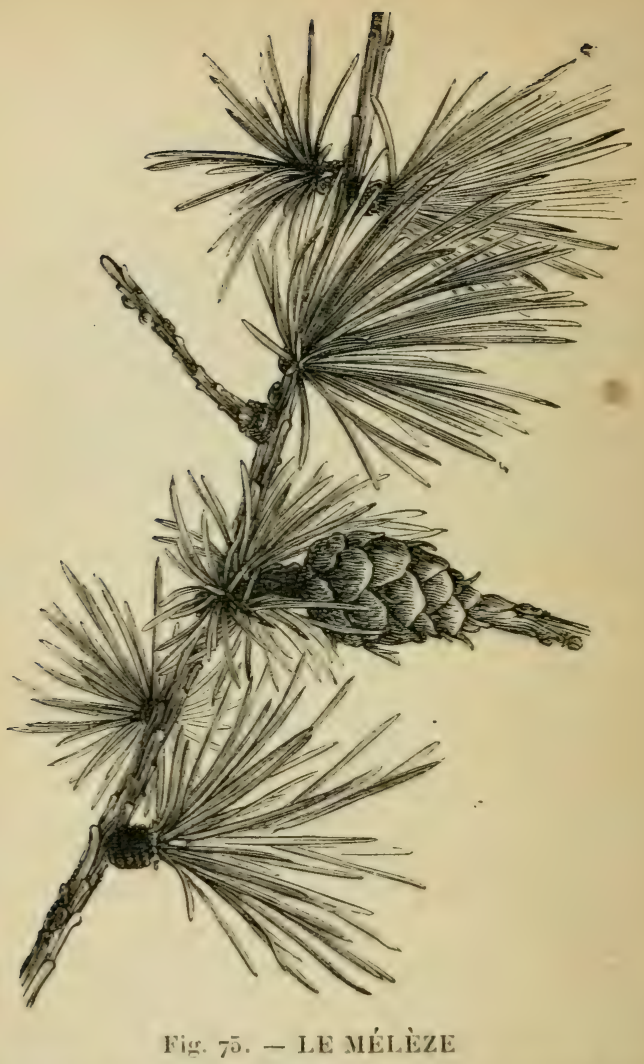

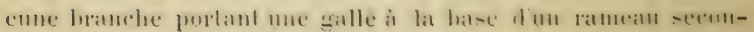
daire. - Fluraisun. mars: malurite. novembre: dir-imination. juin-juillet suivant. 


\section{Fï. 7̄. - LE MÉLĖZE}

Lurix: Europura)

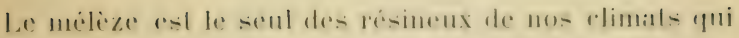

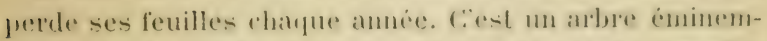
ment montagnard; it ne prosprere qua de hautes altitudes et dans les -ols frais et profonds des vallées suprirecures. Transporte dans les plaines et les monlagurs du centre de la lante, il croit rapidement justuä 3n ans: mais. vers cet ige. il se rourer de mousse of dépérit promptement; sm bois reste mon el spongienx. Dans les Apes, il vil jusun à $3(x)$ ans et prodnit un bois comparable au chène pour la durée et la force.

Les repeuplements naturels de méleze se font part petits bouruets. Il est difticile dobtenir des massils réguliers arec cet airbre, qui ne supporte pas le couvert; aussi ne peut-on lui appliquer le systeme de la futaic pleine. Le mélèze aime à croitre isolé. en pleine lumière: cest larlore qui convient le mieux pour garnir les pailurages des montagnes, car son courert léger laisse rroitre lherbe. Le jardinage est le mode dexploitation qui parait le mieux lui convenir.

On emploie le bois de méleze au chauflage, mais il est surtout précieux pour les ourrages de charpente et de menuiserie. 

TABLE DE CUBAGE 



\section{TABLE DE CUBAGE}

felle lable domne le rolume reel de- arbes abaltus. mais non equarris. Elle peut servir au cubage des rhahlis, des arbres a vendre dans les rompes déji exploités. mais on ne pourrait pas liemployer a l'estimation darbres sur pied ; car les calculs sont bissés sur la circonterence moyeme. rirconférence qui ne peut ètrems-uréesi larbre n'a pas été préalablement abattu.

Comme il est admis par le service de la marine que la mesure des longueurs se comple en nombres pairs de lécimètres, el que les circonférences sont mesurées en lécimètres. on a formé la table en calculant, daprès ce morle de mesurage, pour tontes les grosseurs, depuis 1 rentimètre jusquà q mètres, le volume des trongons de 1 a 25 mètres. Mais pour éviter de lui domner trop' détendue, on sest borné a indiquer une fois pur chaque circonférence le volume de 2 a s décimetres.

Il résulte de celle disposition que la lable ne fail connaitre immédiatement que le volume des pièces dont la longueur se compte par mètres. Si la mesure de la longueur comprend des décimètres, il faut ajouter au chifre yui correspond à la longueur en métres celui qui correspond aux décimètres.

$\mathrm{Si}$, par exemple, on reut comnaitre le rolume diue pièce dont la circonférence au milieu est de $\mathrm{I}^{\mathrm{m}}, 3$ et la longueur $11^{\mathrm{m}} \cdot 6$. on cherchera dans la $\mathrm{j}^{\mathrm{r}}$ colonne le nombre 11 qui représente la longueur en mètres: puis. suivant la ligne horizonlale jusrguà la colonne en tèle de 
laquelle est inscrite la circonférence $1^{\mathrm{m}}, 3$, on trouvera le nombre $1^{\mathrm{m}}, 4793$, qui représente le volume d'un cylindre de $1^{\mathrm{m}}, 3$ de tour et de 11 mètres de longueur.

On cherchera ensuite dans la colonne des longueurs le chiffre 9.6 , et le nombre correspondant 0,0807 donnera le volume du cylindre de $1^{\mathrm{m}}, 3$ de tour sur $\mathrm{o}^{\mathrm{m}}, 6$ de longueur. On ajoutera les deux nombres 1,4793 et $0,080-$ et le total 1.5600 représentera le volume cherché,

Convensiox des prix. - Le volume en bois diceuvre indiqué dans la table est le volume en grume, mais comme les usages commerciaux ont fait préférer à ce rolume réel, facile à apprécier et à déterminer, des cubages fictifs, dits au 1/4. au 1/5, au 1/6, qui sont réputés devoir faire connaitre la quantité de marchandise réellement utilisable, il est important de saroir calculer ce que vaut le mètre cube en grume, unité que nous avons adoptée, quand on connait le prix du mètre cube, au quart sans déduction, au cinquième, ou au sixième déduit.

Pour obtenir le prix du mètre cube en grume, ètant donné le prix du mètre cube au quart sans déduction, il suffira de multiplier ce dernier prix par 0,785 .

Pour obtenir le prix du mètre cube en grume, étant donné le prix du mètre cube au cinquième déduit, il faudra multiplier ce dernier prix de 0,502 , ou plus simplement prendre la moitié. S'il s'agit enfin de passer du prix du mètre cube au sixième déduit à celui du mètre cube en grume, on multipliera le premier par 0,545 . 


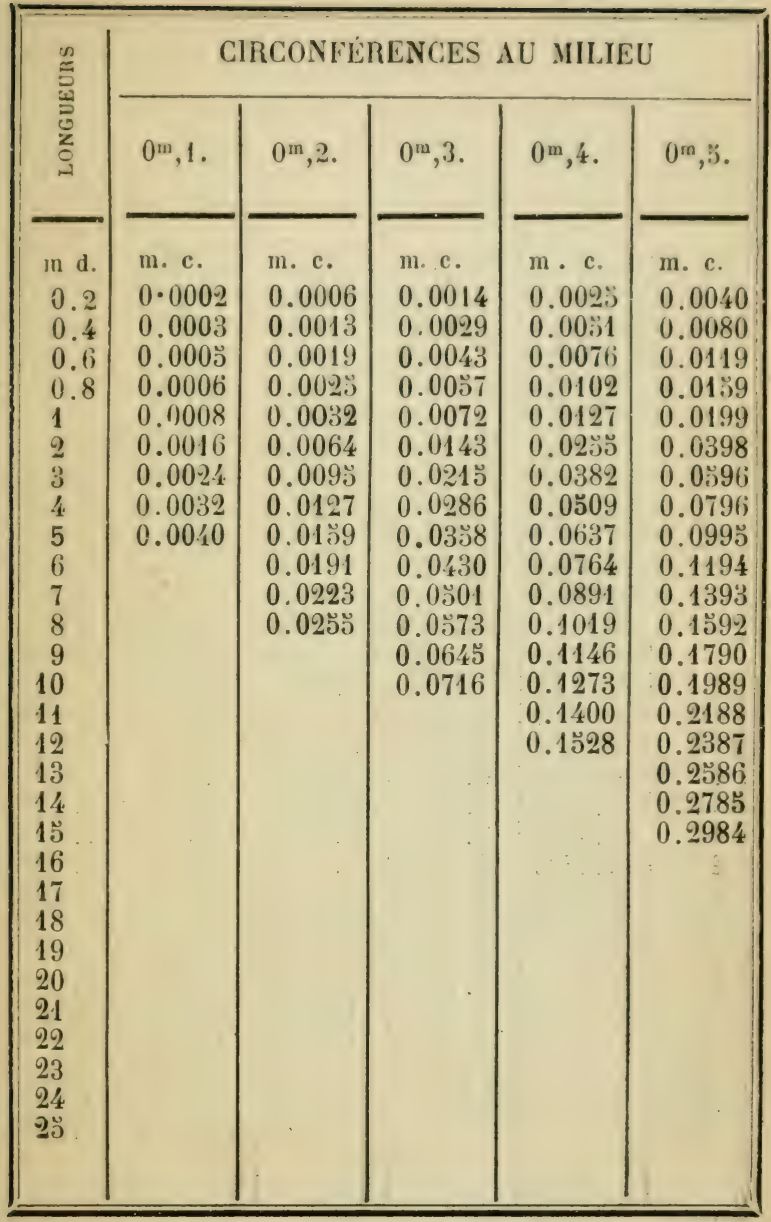




\begin{tabular}{|c|c|c|c|c|c|}
\hline \multirow{2}{*}{ 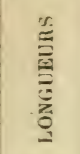 } & \multicolumn{5}{|c|}{ CIRCONFÉREYCES AU MILIEL } \\
\hline & $0^{\mathrm{m}}, 6$ & $0^{\mathrm{m}}, 7$. & $0,{ }^{\mathrm{m}} 8$. & $0^{\mathrm{m}}, 9$. & $1^{\mathrm{n}}, 0$ \\
\hline m.d.: & m. c. & it. c. & m.e. & m. c. & 6. \\
\hline 0.2 & 0.0057 & 0.0078 & 0.0102 & 0.0129 & 0159 \\
\hline 0.4 & 0.011 วั & 0.0156 & 0.0204 & 0.0258 & 0.0318 \\
\hline 0.6 & 0.0172 & 0.0234 & 0.0306 & 0.0387 & 0.0477 \\
\hline 0.8 & 0.0229 & 0.0312 & 0.0407 & 0.0316 & 0.0637 \\
\hline 1 & 0.0286 & 0.0390 & 0.0509 & 0.0645 & .0796 \\
\hline 2 & 0.0573 & 0.0780 & 0.1018 & & 0.1592 \\
\hline 3 & 0.0859 & 0.1170 & 0.1528 & 0.1 & 0.2387 \\
\hline 4 & 0.1146 & 0.1500 & 0.2037 & 0.2 & 0.3783 \\
\hline כ & 0.1432 & 0.1950 & 0.9316 & 0.32 & 0.3979 \\
\hline 6 & 0.1719 & 0.2340 & 0.3016 & 0.38 & 0.4775 \\
\hline 7 & 0.2003 & 0.2 & 0.32 & 0.4 & 0.5370 \\
\hline 8 & 0.2292 & 0.3119 & 0.4074 & 0.5137 & 0.6366 \\
\hline 9 & 0.2378 & 0.3509 & 0.458 ใ & 0.5801 & 0.7172 \\
\hline 10 & 0.2863 & 0.38 & $0 .)$. & 0.6146 & 0.7958 \\
\hline 11 & 0.3151 & 0.42 & 0.5602 & 0.7091 & 1.8754 \\
\hline 12 & 0.3438 & 0.46 & 0.6112 & & $0.9 \% 49$ \\
\hline 13 & 0.3724 & 0.50 & 0.6 & 0.8 & 1.0343 \\
\hline 14 & 0.9011 & 0.5459 & 0.7130 & $0.902 i$ & 1.1141 \\
\hline 13 & 0.4297 & 0.8849 & 0.7639 & 0.9671 & 1.1937 \\
\hline 16 & 0.4384 & 0.62 & 0.8148 & 1.0 & 1.2732 \\
\hline 17 & 0.4870 & $0 .(1629$ & $0.864 i$ & 1.0961 & 1.3328 \\
\hline 18 & $0.51:$ & 0.7019 & 0.9167 & $1.160 \ddot{3}$ & 1.4324 \\
\hline 19 & & 0.7409 & 0.9676 & 1. 2230 & 1.51120 \\
\hline 20 & & 0.7799 & 1.0186 & $1.289 \%$ & 1.0915 \\
\hline 21 & & & 1.0195 & $1.33 \% 0$ & 1.6711 \\
\hline 22 & & & $1.120^{4}$ & 1.4185 & 1.7507 \\
\hline 23 & & & & 1.4830 & 1.8303 \\
\hline 24 & & & & 1.5475 & 1.9098 \\
\hline 23 & & & & 1.6120 & 1.9894 \\
\hline & & & & & \\
\hline
\end{tabular}




\begin{tabular}{|c|c|c|c|c|c|}
\hline \multirow{2}{*}{ 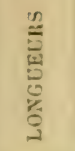 } & \multicolumn{5}{|c|}{ CIRCONFERENCES AU MIIEU } \\
\hline & $1^{m}, 1$. & $1^{\mathrm{m}}, 2$ & $1^{m}, 3$ & $1^{m}, 4$ & $1^{m+1,8 .}$ \\
\hline m.i. & m. $c$. & $m e$. & m. e. & m. e. & in. e. \\
\hline () 2 & 0.01133 & 0.11229 & 0.0269 & 0.0312 & $0.03 \mathrm{is}$ \\
\hline 0.4 & $0.038: i$ & 0.04 .58 & 0.0 .338 & $0.06 i 24$ & $0.0716 i$ \\
\hline 0.6 & $0.0 \div 38$ & 0.0688 & 0.0807 & 0.09936 & 0.1197 \\
\hline 0.8 & 0.0770 & 0.09 & 0.1076 & $0.19 \div 8$ & \\
\hline 1 & 0.0963 & 0.1146 & 0.134 .5 & $0.15 i(10$ & 0.1790 \\
\hline y & 0.1926 & 0. 2292 & 0.2690 & 0.3119 & $0.3 \% \times 1$ \\
\hline 3 & 0.2988 & 0.3438 & $0.403{ }^{\circ}$ & 0.41 & c.83:371 \\
\hline 4 & $0.38 \% 2$ & 0.4384 & 0.5379 & $0.6 .=$ & (1).716.2 \\
\hline$\ddot{g}$ & $0.181 \%$ & 0.57 & 0.6724 & $0 . \bar{\tau}$ & 0.89 .93 \\
\hline 6 & 0.377 & 0.(187:) & 0.8069 & $0.93 \% 8$ & 1.074 .3 \\
\hline 7 & 0.6710 & 0.80 & 0.94 & 1.0 & 1. 283.3 \\
\hline 8 & 0.7703 & $0.916 \overline{1}$ & 1.07 .59 & 1.2 & 1.4321 \\
\hline 9 & $0.8666^{\circ}$ & 1.0313 & 1.2104 & 1.4037 & 1.6114 \\
\hline 10 & 0.9629 & 1. 1159 & 1.3148 & 1.つั: & $1.790 \%$ \\
\hline 11 & 1.0992 & 1. $260 \%$ & 1.4793 & 1.7157 & $1.969 \%$ \\
\hline 12 & 1.15i.i & 1.3731 & 1.6138 & 1.8717 & $2.148 \%$ \\
\hline 13 & 1.2 .18 & 1.48 & 1.74 & 2.0277 & $2.327 \mathrm{i}$ \\
\hline 14 & 1.3180 & 1.6013 & 1.8828 & $2.18: 36$ & 2.5065 \\
\hline $1: 5$ & 1.4443 & 1.7189 & 2.0173 & 2.3396 & 2.6857 \\
\hline 16 & $1 . \breve{4} 406$ & 1.833i & 2.15 & 2.4 & $2.86)+8$ \\
\hline 17 & 1.6369 & 1.9481 & 2.2862 & 2.6 .516 & $3.0 \div 38$ \\
\hline 18 & 1.7332 & $2.062 i$ & 2.4207 & 28076 & 3.2228 \\
\hline 19 & $1.839 \%$ & 2.1772 & $2.3 \%$ & 2.96 & 3.4019 \\
\hline 20 & 1.92: & 9.29 & 2.68 & 3.11 & 3.3810 \\
\hline 21 & 2.0221 & 2.40 & 2.82 & 3.27 & \\
\hline 22 & 2.1181 & 2.5210 & 2.93 & 3.1314 & 3.9391 \\
\hline 23 & 2.21 & 2.63 & 3.0 & 3.58 & 4.1181 \\
\hline 21 & 2.31 & 2.78 & 3.2 & 3.7 & +.2972 \\
\hline 28 & 2.1072 & 2.8648 & 3.3621 & 3.8993 & 4.4762 \\
\hline
\end{tabular}




\begin{tabular}{|c|c|c|c|c|c|}
\hline \multirow{2}{*}{ 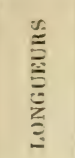 } & \multicolumn{5}{|c|}{ CIRCONFÉREYCES AL MILIEL } \\
\hline & $1^{\mathrm{m}}, 6$ & $1^{\mathrm{m}}, 7$ & $1^{\mathrm{m}}, 8$. & $1^{\mathrm{m}}, 9$ & $2^{m}, 0$ \\
\hline m.d. & m. c. & m. c. & m. c. & m. c. & m. r. \\
\hline 0.2 & 0.0407 & 0.0460 & 0.0316 & $0.05 ั$ ๐ั & 0.0637 \\
\hline 0.4 & 0.0815 & 0.0920 & 0.1031 & 0.1149 & 0.1273 \\
\hline 0.6 & 0.1222 & 0.1380 & $0.15 \div 7$ & $0.172+$ & $0 \quad 1910$ \\
\hline 0.8 & 0.1630 & 0.1840 & 0.2063 & 0.2298 & 0.2546 \\
\hline 1 & 0.2037 & 0.2300 & $0.25 \% 8$ & 0.2873 & 0.318 .3 \\
\hline 2 & 0.4074 & 0.4600 & $0.31: 7$ & $0.57+6$ & 0.6366 \\
\hline$\overline{3}$ & 0.6112 & 0.6899 & 0.773 .5 & 0.8618 & $0.93 \div 9$ \\
\hline 4 & 0.8149 & 0.9199 & 1.0313 & 1.1491 & 1. 2732 \\
\hline$\ddot{3}$ & 1.0186 & 1.1499 & 1. 28.12 & $1.436 !$ & 1.3913 \\
\hline (i & 1.2223 & 1. 3799 & $1.5 \pm 70$ & 1.7236 & 1.9099 \\
\hline 7 & 1.4260 & 1. 6099 & $1.80+8$ & 2.0109 & 2. 2282 \\
\hline 8 & 1.6397 & 1. 8398 & 2.0626 & 2. 2982 & 2.5460 \\
\hline 9 & 1.8333 & 2.0698 & 2.3205 & ‥ วั8วัว & 2.8648 \\
\hline 10 & 2.0372 & 2. 2998 & 2.วั 83 & 2.8727 & 3. $18: 31$ \\
\hline 11 & 2.2409 & 2. 298 & 2.8361 & 3.1600 & 3.5014 \\
\hline 12 & $2 .+446$ & 2.7อง & $3.09 \div 0$ & $3.4 \pm 73$ & 3.8197 \\
\hline 13 & 2.6483 & 2.9897 & 3.3318 & 3.7346 & 4.1380 \\
\hline 14 & 2.8321 & 3. 2197 & 3.0046 & 4.0218 & $4.40(6)$ \\
\hline 13 & 3.05058 & 3.4497 & 3.8675 & 4.3091 & 4.7746 \\
\hline 16 & 3. 2อั9วั & 3.6797 & 4. 1 2ั3 & +. & วั.0930 \\
\hline 17 & 3.4632 & 3.9096 & 4.3831 & 4.8837 & i. 4113 \\
\hline 18 & 3.6670 & 4.1396 & $4.6+10$ & :. 1709 & З. \\
\hline 19 & 3.8706 & 4.3696 & 4. .488 & 3.4382 & $6.0 \div 79$ \\
\hline 20 & $4.074 t$ & 4. 6996 & o. 15ibio & 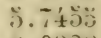 & (i. 3662 \\
\hline 21 & 4. 2781 & 4.8296 & วั.414う & 6. 0328 & 6.6845 \\
\hline 22 & 4.4818 & 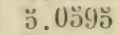 & ว้. 6723 & 6.3200 & 7. 0028 \\
\hline 23 & ๕. 68 วัว & ร. 2895 & วั. 9301 & 6.6073 & $7.321 \mathrm{i}$ \\
\hline 24 & 4.8892 & อั. & 6.1879 & 6.8946 & 7.6494 \\
\hline 23 & ¿.093! & อ. 7490 & $6.4 \div 38$ & 7.1819 & 7.9577 \\
\hline
\end{tabular}




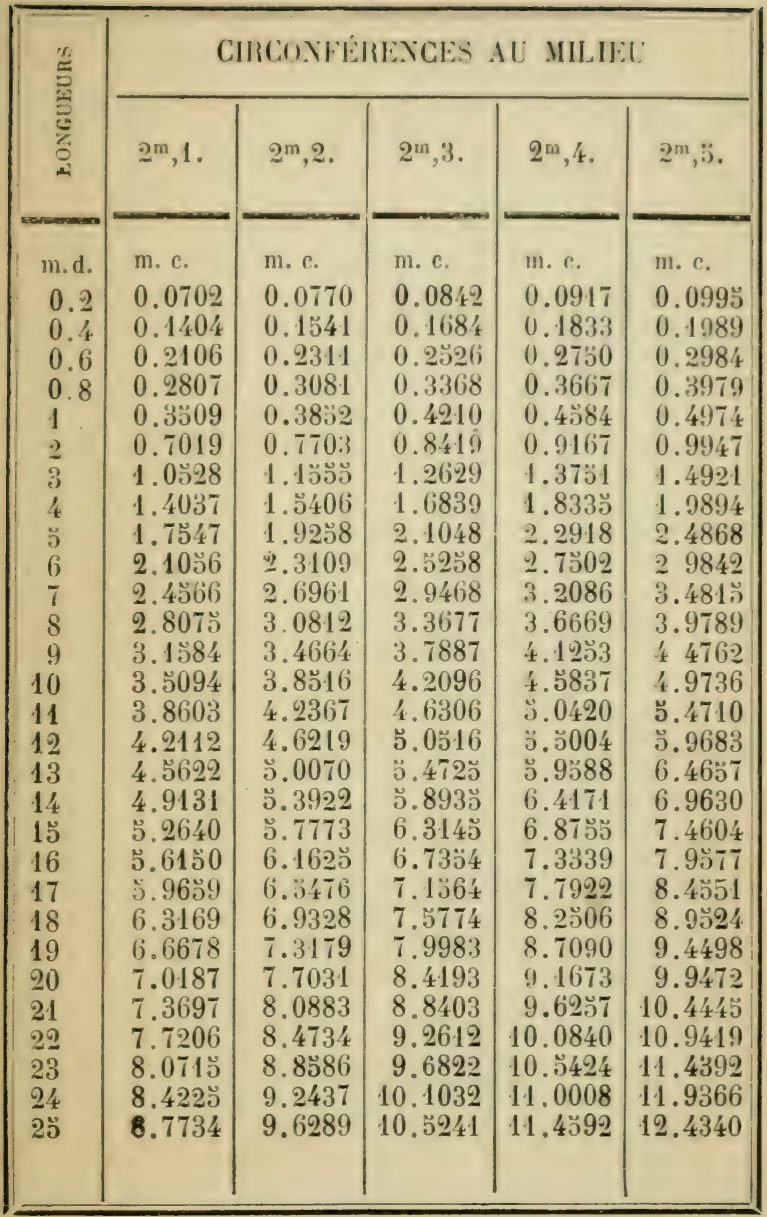




\begin{tabular}{|c|c|c|c|c|c|}
\hline \multirow{2}{*}{ 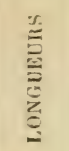 } & \multicolumn{5}{|c|}{ CIRCONFERENCES AL MILIEU } \\
\hline & $2^{\mathrm{m}}, 6$ & $2 \mathrm{~m}, \mathrm{i}$ & $2^{\mathrm{m}}, 8$ & $2^{\ln }, 4$ & $3^{\mathrm{m}}, 0$. \\
\hline m.d. & m. e. & m $c$. & m. $r$. & m. r. & ‥ c. \\
\hline 0.2 & 0.1076 & $0+1160$ & 0.1248 & 0.1338 & 14332 \\
\hline $0 . \overline{4}$ & 0.2152 & (1) 2320 & 0.2496 & 0.2677 & \\
\hline 0.6 & 0.3228 & $03 \$ 81$ & 0.3743 & 0.4015 & $\left\{\begin{array}{l}49(2) 7 \\
\end{array}\right.$ \\
\hline 0.8 & 0.4304 & 0.4641 & 0.4991 & 0.5 & \\
\hline 1 & 0.3379 & 0.0801 & 0.6239 & 0.1 & \\
\hline 2 & 1.07 & 1. 16 & 1.2478 & 1.3 & $1.432 \%$ \\
\hline 3 & 1.6139 & 1.7404 & 1.8717 & 2.0077 & $i 56 i$ \\
\hline 4 & 2.1518 & 2.320 .3 & $2.495 \%$ & & $2.86 \div 8$ \\
\hline$\ddot{j}$ & 2.6897 & 2.9006 & 3.1194 & 3.3 & 3.5810 \\
\hline$\ddot{i}$ & 3.2277 & 3.4807 & 3.7433 & 4.0153 & 4.2932 \\
\hline 7 & 3.76 .36 & 4.06 & 4.3 & 4.68 & 3.0114 \\
\hline 8 & 4.3033 & 4.64 & 4.9911 & 3.3840 & 3.7296 \\
\hline 9 & 4.8413 & $\check{0.9}$ & כ̈.til & 6.0232 & 6.4tios \\
\hline 10 & 8.3794 & ๖. 80 & 6.2389 & (i. 6 & 7.1620 \\
\hline 11 & 30.9174 & 6.3813 & 6.8628 & 7.3617 & 7.8782 \\
\hline 12 & 6.4533 & 6.9 & 7.4866 & 8.0310 & 8. .) 144 \\
\hline 13 & (i. $999 \mathrm{3} 3$ & 7. Silli & 8.1100 & 8.7 & 9.3106 \\
\hline 14 & 7.5912 & 8.12 & 8.7344 & 9.3095 & 10.0268 \\
\hline $1: i$ & 8.06 & 8.7 & 9.30 & 10.0387 & 10.7430 \\
\hline 16 & 8.6071 & 9.2819 & 9.9822 & 10.7180 & $11.4 \div 92$ \\
\hline 17 & 9.1450 & 9.8620 & 10.6060 & 11.3772 & 12.1754 \\
\hline 18 & 9.6830 & 10.4422 & 11.2300 & 12.0464 & 12.8915 \\
\hline 19 & 10.2209 & 11.0223 & 11.8339 & 12.7157 & 13.6077 \\
\hline 20 & 10.7589 & 11.6024 & 12.4777 & 13.3849 & 14.3239 \\
\hline 21 & 11.2968 & 12.1820 & 13.1016 & 14.0312 & 15.0601 \\
\hline 22 & 11.8348 & 12.7626 & 13.7250 & 14.7234 & 1.. 7663 \\
\hline 23 & 12.37 & 13.34 & 14.3494 & 13.3927 & 16.4793 \\
\hline 24 & 12.9106 & 13.9209 & 14.9733 & 16.0619 & 17.1887 \\
\hline 23 & 13.4486 & 14.5030 & 13.5972 & 16.7312 & $17.90 \% 9$ \\
\hline
\end{tabular}




\begin{tabular}{|c|c|c|c|c|c|}
\hline \multirow{2}{*}{ 岁 } & \multicolumn{5}{|c|}{ CIRCONFLRENCES AU MHIEL } \\
\hline & $3^{\mathrm{m}}, 1$. & $3^{\mathrm{m}} \cdot 2$ & $3^{m} \cdot 3$ & $3^{m} \cdot 4^{6}$ & $3^{m} . .$. \\
\hline m. 1. & m. c. & III. r. & III. c. & 111. e. & in. $c$. \\
\hline 0.2 & $0.1 \% 29$ & $0.1631)$ & $0.17: 33$ & $0.18: 0$ & (1). $19 \% 0$ \\
\hline 0.4 & 03059 & $0.32 \% ?$ & $0.2 ; 6$ ib & 0.5680 & 1).3839 \\
\hline $0.1 ;$ & 0.4388 & 0.4889 & $0 . \therefore 2011$ & $0.83 i ! !$ & $0 . \check{849}$ \\
\hline 0.8 & 0.6118 & $0.6 \mathrm{i} 19$ & $0.6(9: 33)$ & $0.73, \ldots ! !$ & 0.7799 \\
\hline 1 & 0.7617 & 1).8149 & 0.8 fififi & $0.91 ! 1 !)$ & (1). .9748 \\
\hline 2 & 1.5295 & 1. .0297 & 1.7332 & $1.8: 398$ & 1. $.9 ! 94 ;$ \\
\hline$\pi$ & 2.2912 & 2.4446 & บ. 5998 & $2.7 ., 97$ & 2.9245 \\
\hline 4 & $3.0 \% 90$ & 3. 2วั9อั & 3.4664 & $3.67 ! 7$ & 3.8993 \\
\hline$\ddot{3}$ & 3.8237 & 1.0744 & 4.3230 & 4. 89966 & 4.8741 \\
\hline 6 & 1. & 4.8892 & ว. 1996 & כ้.5ั19̋̈ & วั. 8489 \\
\hline 7 & i. 3532 & 5.7041 & 6.06602 & 6.1394 & 6. 8238 \\
\hline 8 & 6.1179 & (i. 5190 & 6.9328 & 7.3699 & т.7986 \\
\hline 9 & 6.8827 & T.\$3339 & 7.7994 & 8. $27 ! 9$ & 8.7734 \\
\hline 10 & 7.6474 & $8.148 i$ & 8.6660 & 9.1942 & !). 7482 \\
\hline 11 & 8.4121 & 8.9036 & 9. .อว326 & 10.1191 & 10.7231 \\
\hline 12 & 9.1769 & 4.7785 & 10.3992 & 11.03900 & 11.6979 \\
\hline 13 & 9.9416 & 10.5้934 & 11.2658 & 11.9589 & 12.6797 \\
\hline 11 & 10.7064 & 11.4082 & 12.1324 & 12.8788 & 13.6475 \\
\hline $1 \ddot{3}$ & 11.4711 & 12.2231 & 12.9990 & 13.7987 & 14.6294 \\
\hline 16 & 12.2338 & 13.0380 & 13.8636 & 14.7187 & 13.5972 \\
\hline 17 & 13.0006 & 13.8528 & 14.7322 & 13.6386 & 16.5720 \\
\hline 18 & 13.7653 & 14.6677 & 1อ. อ̈988 & $16 . ว ั 58 \%$ & 17.3468 \\
\hline 19 & 14.53301 & 13.4826 & 16.4654 & 17.4784 & 18.5217 \\
\hline 20 & 15.99 .8 & 16.2975 & 17.3320 & 18.3983 & 19.4963 \\
\hline 21 & 16.0595 & 17.1123 & 18.1986 & 19.3182 & 20.4743 \\
\hline 22 & 16.8243 & 17.9272 & 19.0632 & 20.2381 & 21.2261 \\
\hline 23 & 17.5890 & 18.7421 & 19.9318 & 21.1381 & 2.2 .4210 \\
\hline $2 x$ & 18.3อ38 & 19.วัธิ & 20.7984 & 22.1780 & 23.39ว8 \\
\hline 25 & 19.1185 & 20.3718 & 21.6650 & 22.9979 & $2 ' .9979$ \\
\hline
\end{tabular}




\begin{tabular}{|c|c|c|c|c|c|}
\hline \multirow{2}{*}{ 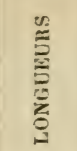 } & \multicolumn{5}{|c|}{ CIRCONFÉRENCES AU MILIEU } \\
\hline & $3^{\mathrm{m}}, 6$ & $3^{\mathrm{m}}, 7$ & $3^{\mathrm{m}}, 8$ & $3^{\mathrm{m}}, 9$ & $4^{\mathrm{m}}, 0$ \\
\hline m. d. & m. c. & m. c. & $\mathrm{m}$. & $\mathrm{m}$. & $\mathrm{m}$. \\
\hline 0.2 & 063 & 0.2179 & 298 & 0.2421 & 346 \\
\hline 0.4 & 125 & 0.43358 & 96 & 0.4841 & 093 \\
\hline 0.6 & 0.6188 & $0.65 ั 36$ & 0.6895 & .79 & \\
\hline 0.8 & $0.820 ั \mathrm{I}$ & $0.8 \mathrm{i} 1 \mathrm{o}$ & 0.9163 & 0.9683 & 186 \\
\hline 1 & 1.0313 & $1.089 \%$ & 1.1491 & 1.2 & \\
\hline 2 & 2.0626 & 2.1783 & 2.2982 & 2.4 & \\
\hline 3 & 3.0940 & 3.2682 & 3.4473 & 3.6314 & 3.8187 \\
\hline 4 & 4.1 & $4.3 \mathrm{~s}$ & & & \\
\hline$\ddot{3}$ & วั. 1566 & อ.4471 & อ. 7450 & 6.0 & 62 \\
\hline 6 & 6.18 & 6.3363 & 6.88 & 7.9 & \\
\hline$\tau$ & 7.2193 & 7.62 & 8.0 & 8.4726 & 27 \\
\hline 8 & 8.2 .06 & 8.71303 & 9.1928 & 9.6830 & 10.1859 \\
\hline 9 & 9.2819 & 9.80 & 10.3419 & 10.8 & 11. \\
\hline 10 & 10.3132 & $10.89 \div 2$ & 11.4 ! & 12.1 & 12.7324 \\
\hline 11 & 11.3446 & 11.9836 & 12.6401 & 13.3 & 14.0056 \\
\hline 12 & 12.37 & 13.0730 & 13.7 & $14.3:$ & 13. \\
\hline 13 & 13.4072 & $\left.14^{\prime} .162\right\}$ & 14.9383 & 15.7349 & 16. วั5ฐ1 \\
\hline 14 & 14.4385 & 15.23018 & 16.0874 & 16.9402 & 17.8254 \\
\hline $4 \breve{3}$ & 15.4 & 16.3412 & 17. 2365 & 18.1อัว 6 & 19. \\
\hline 46 & 16.5012 & 17.4306 & 18.3856 & 19.3660 & 20.3718 \\
\hline 17 & $17.5320 ั$ & 18.5201 & $19.0 ั 3$ & 20.3763 & 916101 \\
\hline 18 & 18.5638 & 19.6093 & 20.68 & $21.786 \pi$ & 22.9133 \\
\hline 19 & 19.5952 & 20.69 & & 22.9971 & 2't.1916 \\
\hline 20 & 20.6 & 21.78 & 29.8 & 21 & 2:.46!8 \\
\hline 21 & 21 & 22.877 & $2^{\prime} .1311$ & 20.11 & 26.7380 \\
\hline 22 & 22.6891 & 23.95 & & 26.6 & 28.0143 \\
\hline 23 & 23.7203 & 25.0566 & 26.4293 & 27.8386 & 29.2840 \\
\hline 24 & & 26.1460 & $27.578 t$ & 29.0490 & 30.5578 \\
\hline 20 & 20.7831 & $27.233 \mathrm{4}$ & 28.727 & 30.2593 & 31.8310 \\
\hline
\end{tabular}




\section{TABLE ALPHABÉTIOUE}

DES MATIRES CONTENUES DANS LE VOICWE

Abatage des futaies, 1/8. - des taillis, 109 .

Acide azotique, 23.

- carbonique, 10.

Aération de l'eau, 23.

Affectations(formation des), 1 亿3.

$\Lambda$ ge des arbres (déterminalion de l'), 61.

Alevin de saumon, 256.

Alluvions (sols d'), 25.

Altitudes de quelques points du globe, 4 .

Amélioration (coupes d'), 148.

Aménagement des taillis, 105.

Aménagement des fulaies, 145.

Ammoniaque, 23.

Anciens, 97.

Anthères, 84.

Appâts empoisonnés, 232.

Arable (sol), 24.

Argile, 28.

Arpentages, 105, 194 .

Assainissement, 184 .

Atmosphère (composition de $\left.\mathrm{l}^{\prime}\right), 9$.

Aubier, 64.

Aune (floraison), 85.

- rameau arec chatons, 282.

Azote, 10.

Balivages, 197 .

Baliveaux (définition), 96. (choix des), 117.

Ba riges (construction des), 187.

Basaltes, 25.
Baltues, 229, 231 .

Belettes, 233 .

Bèles à cornes, 21 .

Bètes à laine, 218 .

Blanc-étoc(coupes à), $96,15 \mathrm{~g}$.

Bois d'ouvriers. 11\%.

Bombyx du pin, 24o.

Bostriches, 237 .

Bouleau ; graine, 38.

- récolte el semis, 167.

- rameau avec chatons, 278.

Bourgeon, 49, 81 .

Boutons, 49. 79 .

Boutures, 79 .

Cadels, 97.

Calcaires (roches), 27.

Cambium, 46, 56.

Carbone, 11 .

Causes de destruction des poissons, 247 .

Cellules, 41.

Cerf (le), 222 .

Charme ; floraison, 84, 86.

- graine, 90.

- taillis de), 104.

- futaies, 147.

- rameau fructi fère, 268 .

Chatons du chêne, 84 . du charme, 84 .

Chatons de l'aune, 85.

- des pins et sapins, 85.

Chats sauvages et domestiques, 234 .

Chêne ; germination, 37 .

- couches annuelles. 60. 
Clicine; lloraison, 8f, s6.

- fructification, 89 .

- rourre, 262.

Chèvres. 220.

Chevreuil (le), 224,

Circulation de la sève. 51 .

Composition de l'atmos. phère, 9.

Composition de l'eau, 16.

Corniers, 195.1 du bois, 11 .

Cotylédons, 38 .

Coupes (division en), 101.

- d'ensemencement, 135.

- secondaires, 136.

- définitives, 137.

- d'amélioration, 138.

Courtilière (la), 243.

Couvert (effet du), 67.

Criées, 211.

Cubage différents modes de), 205 .

Cuticule, 43 .

(tarifs de), 30 s.

Daim (le), 223.

Décrépitude des arbres, 70 .

Défensabilité, $217,219$.

Définitives (coupes). 137.

Défrichement (effetsdu), 3\%.

Dimensions du globe terrestre, 1.

Drageons, Sa.

Dytiques, 2 f.

Eau ; sa composilion. 16.

- douce, salée, pluviale, '2'.

Eclaircies, 138.

Ecorce; formation, bi.

Ecrevisse, 258.

Ecureuil (1'), 229 .

Elfels du défrichement. 32.
Effels du pailurage, 33.

Embryon, 89 .

Ensemencement coupesd . 135.

Entretien des taillis, 11 (1.

Epiderme, 43.

Epicéa, rameau à lleurs màles, 292.

Erable champètre; graine.

$$
92 .
$$

- rameau florifère, $2-60$.

E-pacement des plants, 176 .

Estimations, 201.

Etamines, 84 .

Exploitabilité, 140.

Exploitation des taillis, 111 .

- des futaies, 19s.

Faconnage, 149 .

Faine; sa récolte, 165.

- conservation, 160 .

Fécondation, 88, 252.

Femelles (fleur's), 8\%.

Fertilité conditions de . 33.

Feuilles, 47.

Fibres, 42.

Filet. 8\%.

Fleur, 83.

Fléves origine des. 17.

Formation des tissus,

Forme de la terre, 1.

Fossés (tracé des), 185.

Fossiles, 3 .

dimensions, 1 St.

Fouines, 233.

Fourlé, 13\%.

Foville, 88 .

Fraie (époque de la), ״̈!).

Fraveres naturelles, 24 ! . artificielles, 2.4.

Frêne ; graine, 91. feuille, 272 .

Futaies, 132.

Furetage, 12J.

$$
\text { (liaute), } 138 \text {. }
$$

Gaulis. 138 . 
Gemmage, 160 .

Germination, 37.

Gibier, 221.

Glaciers, 20.

Glands, 89,164 .

Glissoirs, 150.

Granits, 25.

Graines du hètre, 88.

- du charme, go.

- du pinsylvestre, go, $9^{3}$.

Graines de l'orme, 91 .

- du tremble, 91,

- du frène, 91 .

- de l'érable champètre, 99.

- de l'érable sycomore, 92.

Grefle, 79 .

dusaule marceau, 93 .

Grès, 28 .

Griffage des réserves, 198 .

Hanneton (le), 2 \&1.

Hérisson (le), 231 .

Hermaphrodites (fleurs), 87. Hètre; fleur mâle, 83.

- fleur femelle, 86.

- fruit, 88.

- rameau; 266 .

IIumus (I'); sa formation,29. Hydrogène, 16. sa destruction, 31 .

Hylesine du pin, 239 .

Ignées (roches), 25.

Incubation (appareils $\cdot d^{\prime}$ ), 254.

Insectes, 237 .

Jalons d'arpentage, 195. du récolement, 210 . Jardinage, 15 I.

Jeunesse des arbres, 69 .

Lacs (formation des), 6.

Laies d'aménagement, 108.
Lailance, 253.

Lapin (le), 227.

Liège extraction du), 129.

Lièvre (le), 227.

Lignes de coupes, 108, 190.

Loup (le), 231.

Màles (fleurs), 86.

Maladies des arbres, 74.

Marcottes, 183.

Marnes, 27.

Marteaux, 199.

Martelages, 197.

Maturilé des bois, 72 .

Melèze (futaies de), 155 .

- rameau avec cònes,302

Mesure des dimensions des arbres, 202.

Métamorphiques(roches), 25

Méthode naturelle, 133.

Mers, 17.

Moelle (la), 45 .

Modernes, 96.

Mort des arbres, 70.

Morts-bois, 97.

Mulot (le), 230 .

Neige (formation de la), 19 .

Nettoiements, 119. 138.

Oiseaux (les), 234.

Orme (l'); graine, 91 .

- rameau, 270.

- fleur, 86.

- fruit, 91.

Ovaire, (l'), 85.

Ovule (1'), 85 .

Oxygène $\left(l^{\prime}\right), 10$.

Parois (arbres), $190^{\circ}$.

Pèche (service de la), 246.

Pépinières ; création, 177.

- volantes, 181 .

Perchis, 138.

Période, 145.

Peuplier blanc, rameau, 286 . 
Pin sylvestre; còne, 90.

- rameau àfleurs mâles, 294 .

Pin mugho; rameau à fleurs mâles, 296 .

- maritime; rameau avec cônes, 298.

- laricio; rameau à fleurs mâles, 300 .

Piquets des coupes,195,210. Pistils, 85.

Plantations, $147,172$.

Pluie (formation de la), 18.

Pollen, 88.

Porcs, 220.

Porphyres, 25.

Possibilité, 140,142 .

Préparation du sol, 168.

Putois (le), 233.

Quartz, 25.

Racines, 47.

Rayons médullaires, 44.

Ravinement (le), 33.

Recepage, 177.

Récolements, 209.

Refroidissement du globe, 1 .

Rejets de souche, 82 .

Renard (le), 231.

Repeuplements, 162 .

Reproduction (fonctions de

78 .

Réserves ; choix, 97, 116.

Révolution, 96 . taille, 189 .

Rigoles, 178.

Rivières, 17 .

Roches, 24 .

Sanglier, (le), 225.

Sapin; rameau à fleurs màles, 290.

Sartage (le) à feu couvert, 126.
Sartage(le)à feu courant 126 . Saule marceau : floraison, 87.

- rameau à feuilles, 288 . Schlitte (chemin de), 150.

Secondaires (coupes), 136 . Sédimentaires(roches), 3,25. Semis," 163 .

Sentiers interdits, 191 .

Sève ascendante, 50.

descendante, 24 .

Sols (définition des), $5 \%$.

Soulèvements, 2 .

Sources; leur. orgine, 17.

Stigmate, 85.

Style, 85,

Strychnine, 232.

Sycomore: fleur, 87 .

- fruit, 92 .

- rameauflorifère, 274 .

Talcs, 25.

Taillis, 95 .

- (entretien des), 119. Tige (composition de la), 44, 58.

Tilleul; rameau florifère, 280

Tire et aire, 158 .

Trachytes, 25 .

Tremble; floraison, 91.

- rameau à feuilles. 284 .

Vaisseaux ponctués, rayés, annelés, 42.

Vallées; leur formation, 6.

Vapeur d'eau, 13.

Vidange des bois. 108, 115, 150.

Vie des arbres, 69 .

Vieilles écorces, 97 .

Virées, 198.

[26-12-8. - Tours, imp. E. Arrault el Cie. 
$\mathrm{SD}$

373

B6

1899

Forestry

\section{Bouguet de la Grye, Amédie Elements de sylviculture 10. éd.}

\section{PLEASE DO NOT REMOVE}

CARDS OR SLIPS FROM THIS POCKET

\section{UNIVERSITY OF TORONTO LIBRARY}

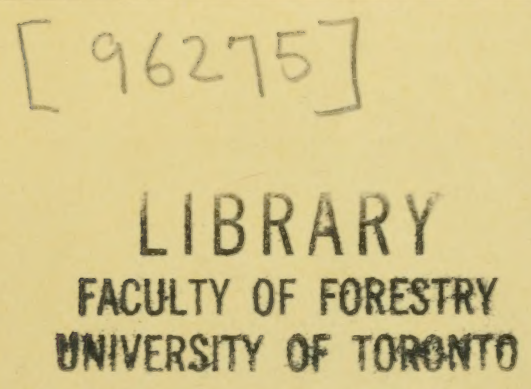


J. R0THSCHTLD, Éditeur, 13, rue des Saints-Pàres, I arìs

M.J. Rothschild a obtenu pour ses belles Publications, ì TExposition universelle de 1878 a Paris: la Croix de la Lér plusieurs Médailles; en to hors Concours, comsi à Vienne, Philadelphie, Bruxelles, Amsterdam, dor, d'Argent, du Mérite et de bon Gour.

\section{EXTR, IT DU}

La Maison du Garde. - Notions d'hygiêne, d'économie domestique et d agrieultme. In voluare orn de 142 gravures, à l'usitee des (iardes forestiers ou particuliers et des petits ménages, var T. Poucos Conservitcur des Forélst. truvoge couronné par la Sorielé rationale dagricuttrie. Prix.

Revue des Eaux ot Forêts. (Annales forestiur.

Journal des interets foresbiats, tom, fluant: Economie forestiere. reb -iscmien!. exploitalion, statistiquo, meres riales, régime des eaux, chasse. louveterie, pisciculture, métallurgie, ele.

L'́gislation et Jurisprudence forestiéres, contenant : Repertoire périod lyue et eritiqu des lois, décrêts, règlements géliéraux, avis et décisions du Conseil d'Etat, arrêtés ministẻriels, cireulaires et décisions adiministratives, arrêts de la Cour de cassation et des Cours d appel, jugements des Tribunaux civils, correctionnels et de commerce, en matière deBois, Forêts, Chasse. Pêche. - Paraissant le to pt le 15 de chaque mois, en dem foscieules de deux ferritet i. -8 .

Prix de faboumenient de is Tievue etdu Ríperloire ersembie. - Pour la France: $15 \mathrm{fr}$ par $: 7$ - Avec 1:Annuaire $18 \mathrm{fr}$. par an. - poir Tetranger : $20 \mathrm{ir}$. - Les abonnements partent du jer janvier. de chaq ie année.

Les Abonnés franeais à $18 \mathrm{fr}$. et les abonnés étrangers a 20 ir recoivent l'Anusira des Baux et Por'ts. Contenant le tablean complet du rersonnel de l'Administration des forêts, du serviee forrestier del'Algérie, la liste des promotions de I'Ecole forestière, le budget et de nombrer , documents statistignes.

Tabie générale des Matières contenues dans les 25 volumes

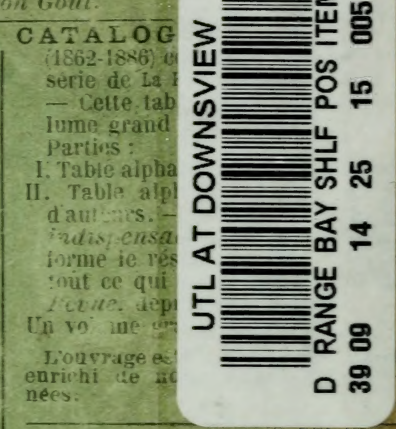

Les Animaux des Forêts. (Naumiferes et 0iseaux), - leur Histoire naturelle. - Chasse á courre. - whasse à tir. - Entretien. - Conservatiou. - Reproduetion. - Zoologio pratique au point de vue de la chasse et de la sylviruiture, par $k$. C.makpit $>$ Soits-inspecieur des foréts. -2 rition. In volume avee 81 graver's relip. . . . 2 ix. $50^{\circ}$

\section{La Chirurgie du Foyer:}

rrate popuirire conterlat inflammations abces, plties, brôtu$\mathrm{P}+\mathrm{s}$, mafadies rirulentes, whears, empnisture:met is, asphyxie.ete. par le 0 ch. Bastcht, A ncien interne des húbilaux. En vo. lame in- 18 avee 45 graviars: reniè en toile..... $3 \mathrm{fr}$, sil

\section{Les Plantes medicinales}

wivifle; de ans ehamps, jardins. furèts. - Deseription et usages des piances crimesbloles, suspecter, veréneusers, vempioyées das la médecine; dans Tindustrie et TĆconomie doinestique, par H. foom. 11. edition. Un volu nue de 500 pages avee 290 gravures; relié......... g. fr.

$$
\text { 26.12-8. - Tours, imp. B. Arranlt et } \mathrm{C} \cdot
$$

